\title{
SEISMIC VELOCITY CHANGES AT WHITE ISLAND VOLCANO, NEW ZEALAND, USING TEN YEARS OF AMBIENT NOISE INTERFEROMETRY
}

\author{
By \\ Alexander Silvanus Yates
}

A thesis

submitted to the Victoria University of Wellington in fulfilment of the requirements for the degree of Master of Science in Geophysics

Victoria University of Wellington

2018 


\section{Abstract}

Seismic velocity changes at volcanoes carry information about stresses present within hydrothermal and magmatic systems. In this thesis, temporal velocity changes are measured at White Island volcano using ambient noise interferometry between 2007-2017. This period contains multiple well-documented eruptions starting in 2012, following an inactive period that extends back over a decade. Three primary objectives are identified: (1) investigate what seismic velocity changes can tell us about dynamic changes beneath the volcano, (2) investigate non-volcanic sources and their possible influence on interpretations, and (3) consider the potential for real-time monitoring using ambient-noise. These objectives extend beyond White Island volcano, with implications for ambient noise monitoring of volcanoes globally.

Two different approaches are used to measure velocity changes at White Island. The first involves cross-correlating noise recorded by pairs of seismic stations. Velocity changes are sought by averaging changes recorded across ten station-pairs that consist of an onshore station and a station on the volcano. The second approach involves cross-correlating the different components of individual seismic stations. This represents a less traditional approach to monitoring volcanoes, but is well-suited to White Island which has one permanent station active throughout eruptive activity. Single seismic stations located onshore are also processed to investigate background regional changes.

Two periods of long-term velocity increases are detected at the volcano. The first occurs during a highly active period in 2012-2013 and the second occurs in the months preceding an explosive eruption in April 2016. Comparison with velocities recorded by onshore stations suggest a meteorological source for these changes is unlikely. Velocity increases are therefore interpreted to reflect cracks closing under increased pressures beneath the volcano. Similarly, a rapid decline in the velocity within 2-3 months of the April 2016 eruption is interpreted to reflect depressurization of the system.

In addition to volcanic sources, we also find clear evidence of non-volcanic processes 
influencing velocity changes at the volcano. Two clear co-seismic velocity decreases of approximately $0.05-0.1 \%$ are associated with a $M_{W} 5.2$ earthquake in 2008 within $10 \mathrm{~km}$ of the volcano - and the $M_{W} 7.1$ East Cape earthquake in 2016. The East Cape earthquake - located $200 \mathrm{~km}$ away from the volcano — produces significant velocity decreases over a large region, as detected by stations onshore and on White Island. This likely reflects dynamic stress changes as a result of passing seismic waves, with an eruption two weeks later interpreted here to have been triggered by this event. Finally, we identify similarities between annual variations recorded by onshore stations and changes at the volcano, suggesting an environmental influence. Velocity changes at White Island therefore represent a complex interaction of volcanic and non-volcanic processes, highlighting the need for improved understanding of external sources of change to accurately detect short-term eruptive precursors. 


\section{Acknowledgements}

Firstly, I would like to thank my primary supervisor Martha Savage. Your guidance over the past year has been invaluable, both with the thesis itself and any general queries I have had. Your dedication to your students is something to aspire to. I also extend my thanks to my external supervisor, Art Jolly, and Corentin Caudron, both of whom have provided encouragement and thought-provoking feedback. To the Westergaard family, I am very grateful for the financial support offered through the Rachael Westergaard Memorial Award. The last year of study has been much easier because of your contribution, allowing me the freedom to focus on my work without financial stress. I also need to thank John Townend, who played an instrumental role in putting my name forward for this award. Similarly, I thank Victoria University for covering a portion of my student fees through the Master's by Thesis Fees scholarship. A big thank you also to GeoNet, through which the majority of data are acquired.

Countless others at Victoria University have helped towards my development at some stage during this thesis, whether this involved having an open door to discuss questions I had or providing thoughtful discussion. In the Geophysics department, past and present, I would like to thank Adrian Bensen, Calum Chamberlain, Francesco Civilini, Dominic Evanzia, Holly Godfrey, Kenny Graham, Chet Hopp, Katrina Jacobs, Jesse Kearse, Andy McNab, Jiten Patel, Sam Taylor-Offord, Konrad Weaver, Jack Whattam and Hubert Zal for your contributions. From the Maths department, I thank Richard Arnold and John Haywood, both of whom made time for me to discuss potential directions I was keen to explore. I would also like to thank Aleksandr Beliaev for help with IT related queries. I am convinced you are the most important person in this department! Also, a big thank you to Patricia Stein, for always being available to answer questions I had about the Masters thesis process.

To my parents, your support throughout my education is more than I could have ever asked for. I owe many of the opportunities I have been afforded in life to you! 
Finally, I would like to extend the biggest thank you to my partner Sascha Zerjal. You have been with me through the long hours and I could not have done this without you. 


\section{Contents}

Abstract ........................... ii

Acknowledgements .......................

List of Figures . . . . . . . . . . . . . . . . . . . . $\mathrm{x}$

List of Tables . . . . . . . . . . . . . . . . . . xiv

1 Introduction $\quad 1$

1.1 Motivation . . . . . . . . . . . . . . . . . . 1

1.2 Primary Objectives . . . . . . . . . . . . . . . . . 2

1.3 White Island Volcano . . . . . . . . . . . . . . . . . . . . . 2

1.3.1 Geological Setting . . . . . . . . . . . . . . . 3

1.3.2 Recent Eruptive History . . . . . . . . . . . . . . . . . . . . . 6

1.4 Study Design . . . . . . . . . . . . . . . . . . . . . 7

1.5 Thesis Layout . . . . . . . . . . . . . . . . . . . 8

1.5.1 General Notes . . . . . . . . . . . . . . . 8

1.5.2 Chapter Summary _. . . . . . . . . . . . . . . . . . . 9 9

1.5.3 Glossary of Terms and Acronyms . . . . . . . . . . . . . . . 10

2 Background Theory 13

2.1 Ambient Noise Interferometry . . . . . . . . . . . . . . . . . . 13

2.1.1 Wavefield Properties . . . . . . . . . . . . . . . . 13

2.1.2 Green's Function Recovery . . . . . . . . . . . . . . . . . 15

2.1.3 Measuring Seismic Velocity Changes . . . . . . . . . . . . 17

2.2 Monitoring Volcanoes with Ambient Noise . . . . . . . . . . . . . . . 20

2.2.1 Physical Mechanism . . . . . . . . . . . . . . . . . 20

2.2.2 Similar Studies . . . . . . . . . . . . . . . . . 20

2.3 MSNoise . . . . . . . . . . . . . . . . . . . . . . . . . . 22

2.3.1 Data Preparation . . . . . . . . . . . . . . . 22

2.3.2 Waveform Preprocessing . . . . . . . . . . . . 23

2.3.3 Computing Cross-Correlation Functions . . . . . . . . . . . 25

2.3.4 Temporal Stacking . . . . . . . . . . . . . . . 27

2.3.5 Relative Travel-Time Variations . . . . . . . . . . . . . . . . . 28 
3 Station-Pair Dataset 33

3.1 Introduction . . . . . . . . . . . . . . . . . . . . 33

3.2 Parameter Justification . . . . . . . . . . . . . . . . . . . . . . . . 34

3.2.1 Identifying Frequencies of Coherent Noise . . . . . . . . . . . 35

3.2.2 Maximising Temporal Stability . . . . . . . . . . . . . . . 37

3.2.3 Moving-Window Cross-Spectrum Parameters . . . . . . . . . . 38

3.2.4 Stacksize Analysis . . . . . . . . . . . . . . . . . . . . . . . . 43

3.2.5 Relationship between SNR and Coherence . . . . . . . . . . . 45

3.2.6 Final Parameter Choices for Velocity Analysis . . . . . . . . . 48

3.3 Results . . . . . . . . . . . . . . . . . . . . . . . . 49

3.3.1 Cleaning and Combining Time Series . . . . . . . . . . . . . 49

3.3.2 Velocity Changes . . . . . . . . . . . . . . . 54

3.3.3 Stability of Measurements . . . . . . . . . . . . . 58

4 Single-Station Dataset $\quad 63$

4.1 Introduction . . . . . . . . . . . . . . . . . . 63

4.2 Parameter Justification . . . . . . . . . . . . . . . . . . . . . 63

4.2.1 Maximising Temporal Stability . . . . . . . . . . . . . . 64

4.2.2 Stack-size Analysis . . . . . . . . . . . . . . . . . . . 66

4.2.3 SNR vs Coherence . . . . . . . . . . . . . . . . . . 68

4.2.4 Final Parameter Choices for Velocity Analysis . . . . . . . . . 70

4.3 Results . . . . . . . . . . . . . . . . . . . . . . 71

4.3.1 Velocity Changes at White Island . . . . . . . . . . . . . . . 72

4.3.2 Comparison with Onshore Velocity Changes . . . . . . . . . 79

4.3.3 Comparison with Tectonic Earthquakes . . . . . . . . . . . . . 81

5 Discussion $\quad 85$

5.1 Sampling Depth and Location . . . . . . . . . . . . . . . . 85

5.1.1 Depth Sensitivity Kernels . . . . . . . . . . . . . 85

5.1 .2 Sampling Location . . . . . . . . . . . . . . . . . . . 86

5.2 Background Sources . . . . . . . . . . . . . . . . . 88

5.2.1 Environmental Factors . . . . . . . . . . . . . . . . . 89

5.2 .2 Tectonic Earthquakes . . . . . . . . . . . . . . . . . . 96

5.3 Volcanic Changes at White Island Volcano . . . . . . . . . . . . . . . 100

5.3.1 Decorrelation Before and During Volcanic Activity . . . . . . 100

5.3.2 Long-term Velocity Increases and Apparant Changes in 2016 . 102

5.3.3 Real-Time Monitoring . . . . . . . . . . . . . . . . . . 107

6 Conclusions 111

6.1 Summary of Findings . . . . . . . . . . . . . . . . . . . 112 
6.1 .1 Volcanic Sources . . . . . . . . . . . . . . . . . 112

6.1 .2 Non-Volcanic Sources . . . . . . . . . . . . . . . . . . . . 112

6.1.3 Suitability of Different Approaches . . . . . . . . . . . . . . 113

6.1.4 Implications for Ambient Noise Monitoring . . . . . . . . . . . 113

6.2 Recommendations for Further Work . . . . . . . . . . . . . . . . 114

A Data Processing Supplementary Material $\quad 131$

A.1 Seismic Station Details . . . . . . . . . . . . . . . . . . . . 132

A.2 Final MSNoise Parameters . . . . . . . . . . . . . . . . . 134

A.2.1 Station-pair Dataset . . . . . . . . . . . . . . 134

A.2.2 Single-Station Dataset . . . . . . . . . . . . . . . 136

A.3 Time Shift Correction (Station-Pairs) . . . . . . . . . . . . . . . . 138

A.4 Correlation Length Influence on Single-Station Processing . . . . . . 140

A.5 Velocity Model for Sensitivity Kernel . . . . . . . . . . . . . . . . . . 142

B Velocity Changes Supplementary Material 143

B.1 Individual single-station component-pairs . . . . . . . . . . . . . . . . 144

B.2 Station-pair WIZ-WSRZ . . . . . . . . . . . . . . . . . . . . 148

B.3 OPRZ station (Single-station Ambient Noise) . . . . . . . . . . . . . 149 


\section{List of Figures}

1.1 Geological Setting of New Zealand Volcanism . . . . . . . . . . . . . 4

1.2 White Island Map . . . . . . . . . . . . . . . . . . . . . 5

1.3 Recent activity timeline for White Island volcano . . . . . . . . . . 6

2.1 Primary and secondary microseisms at White Island . . . . . . . . . 14

2.2 Concept diagram for Seismic Interferometry . . . . . . . . . . . . 16

2.3 Concept diagram for measuring velocity changes using ambient noise 19

2.4 Demonstration of techniques for performing temporal normalisation in MSNoise . . . . . . . . . . . . . . . . . . . . 23

2.5 Demonstration of spectral normalisation . . . . . . . . . . . 24

2.6 Comparison of linear and phase-weighted stacking . . . . . . . . . 25

2.7 Comparison between 1-day and 30-day stacks . . . . . . . . . . . . . 27

2.8 Example of window used to calculate delay times during Moving Window Cross Spectral technique . . . . . . . . . . . . . 28

2.9 Example of performing linear regression on delay times to calculate a velocity change . . . . . . . . . . . . . . . . . . . 31

3.1 Map of station-pairs used for station-pair dataset . . . . . . . . . . 34

3.2 Spectral SNR of station-pair dataset . . . . . . . . . . . . . . . . 36

3.3 SNR computed before and after applying new filters . . . . . . . . . 38

3.4 SNR tests of different pre-processing parameter choices within MSNoise 39

3.5 Residual difference between correlation coefficients measured for different reference stack sizes . . . . . . . . . . . . . . . . . . 41

3.6 Demonstration of noise being added to synthetic cross-correlations . 42

3.7 Recovered velocities from synthetic cross-correlation functions using Moving Window Cross Spectral technique with varied window sizes 43

3.8 Relationship between stack size and correlation coefficient . . . . . . 44

3.9 Demonstration of how a plot of SNR versus Coherence is generated 45

3.10 SNR versus Coherence shown for multiple station-pairs . . . . . . . 46

3.11 Demonstration of how minimum and maximum lag times are chosen for station-pairs . . . . . . . . . . . . . . . . . . 47 
3.12 Plot of 6 months of 30-day stacks for station-pair HAZ-WIZ with chosen windows for velocity analysis shown . . . . . . . . . . . 48

3.13 Moveout plots for all nine component-pairs . . . . . . . . . . . . . . 50

3.14 Demonstration of coherent energy for transverse components . . . . 51

3.15 Demonstration of different station-pairs averaged to calculate a time series for the ZZ component-pair at WIZ station . . . . . . . . . . 53

3.16 Demonstration of smoothing time series using 7 and 14 day moving windows . . . . . . . . . . . . . . . . 54

3.17 Map of station-pairs used in averaged time series . . . . . . . . . . 55

3.18 Velocity changes and correlation coefficients for WIZ and WSRZ time series (station-pair dataset) . . . . . . . . . . . . 56

3.19 FFT of WIZ velocity changes (station-pair dataset) . . . . . . . . 57

3.20 Long-term velocity changes for averaged WIZ and WSRZ time series (station-pair dataset) . . . . . . . . . . . . . . . 5 58

3.21 Average velocity changes after removing individual station-pairs . . 59

3.22 Variance of WIZ averaged time series after removing individual station-pairs . . . . . . . . . . . . . . . . . 60 60

3.23 Stability test of long-term velocity trend of WIZ averaged time series 61

4.1 SNR tests of different pre-processing parameter choices within MSNoise for single-station $0.1-1.0 \mathrm{~Hz}$ ambient noise . . . . . . . . . . 65

4.2 SNR tests of different pre-processing parameter choices within MSNoise for single-station 1.0-2.0 Hz ambient noise . . . . . . . . . . . . . 66

4.3 Comparison of correlation coefficients calculated for 1-day and 30day stacks . . . . . . . . . . . . . . . . . . . 6 67

4.4 SNR versus coherence for auto-correlations and cross-components . 69

4.5 Demonstration of maximum lag times picked for single-station velocity analysis . . . . . . . . . . . . . . . . 70

4.6 FFT of WIZ velocity changes (single-station) . . . . . . . . . . . 72

4.7 Single-station velocity changes and correlation coefficient (CC) measurements for $0.1-1.0 \mathrm{~Hz} \ldots \ldots$. . . . . . . . . . . . . 74

4.8 Long-term trend of $0.1-1.0 \mathrm{~Hz}$ auto-correlations and cross-component velocity changes . . . . . . . . . . . . . . . 75

4.9 Single-station velocity changes and correlation coefficient (CC) measurements for $1.0-2.0 \mathrm{~Hz} \ldots \ldots$. . . . . . . . . . . 77

4.10 Comparison between velocity changes for $0.1-1.0 \mathrm{~Hz}$ cross-components and $1.0-2.0 \mathrm{~Hz}$ auto-correlations . . . . . . . . . . . . . . . 78

4.11 Velocity changes recorded by onshore stations . . . . . . . . . . 79

4.12 FFT of onshore velocity changes (single-station) . . . . . . . . . 80 
4.13 Comparison between velocity changes recorded by $0.1-1.0 \mathrm{~Hz}$ crosscomponents onshore and at White Island. . . . . . . . . . . . . . . . 81

4.14 Velocity changes associated tectonic earthquakes . . . . . . . . . 83

5.1 Depth sensitivity kernels for Love and Rayleigh waves . . . . . . . . 86

$5.2 \quad$ OPRZ-WIZ influence on earthquake signal . . . . . . . . . . . . . 88

5.3 Yearly velocity changes recorded by onshore single-stations . . . . . 90

5.4 Yearly changes in meteorological datasets . . . . . . . . . . . . . 91

5.5 Comparison between meteorological datasets and seasonal 1.0-2.0 $\mathrm{Hz}$ single-station onshore velocity changes . . . . . . . . . . . . . . 93

5.6 Comparison between short-term rainfall and 1.0-2.0 Hz single-station onshore velocity changes . . . . . . . . . . . . . . . . . . . 94

5.7 Comparison between meteorological datasets and seasonal 0.1-1.0 $\mathrm{Hz}$ single-station onshore velocity changes . . . . . . . . . . . . 95

5.8 Analysis of post-seismic relaxation associated with $M_{W} 5.2$ earthquake near White Island . . . . . . . . . . . . . . . . 98

5.9 Evidence of post-seismic deformation following $M_{W} 5.2$ earthquake near White Island . . . . . . . . . . . . . . . . . . . . . . . . 99

5.10 Comparison between single-station correlation coefficients and RSAM data . . . . . . . . . . . . . . . . . . . 101

5.11 Six stages of velocity changes recorded by $0.1-1.0 \mathrm{~Hz}$ cross-correlations at WIZ station . . . . . . . . . . . . . . . . . . . . 104

5.12 Demonstration of seasonal influences at WIZ station and deviations associated with volcanic activity . . . . . . . . . . . . 105

5.13 Centered window versus real-time smoothing . . . . . . . . . 108

A.1 Data availability of seismic stations . . . . . . . . . . . 133

A.2 Demonstration of time shift observed at WIZ station . . . . . . . 138

A.3 Corrected cross-correlation functions showing time shift is no longer present . . . . . . . . . . . . . . . . . . . . 139

A.4 Influence of correlation length on SNR of single-station noise . . . . 140

A.5 Comparison between CCFs after increasing the correlation length . 141

A.6 Velocity model for depth sensitivity kernels . . . . . . . . . . . . . . 142

B.1 Single-station velocity changes for individual 0.1-1.0 Hz auto-correlations 144

B.2 Single-station velocity changes for individual 0.1-1.0 Hz cross-components 145

B.3 Single-station velocity changes for individual 1.0-2.0 Hz auto-correlations 146

B.4 Single-station velocity changes for individual 1.0-2.0 Hz cross-components 147

B.5 Velocity changes and correlation coefficient recorded for station-pair WIZ-WSRZ . . . . . . . . . . . . . . . . . . . . 148

B.6 Velocity changes recorded at OPRZ station (single-station dataset) . 149 


\section{List of Tables}

1.1 Glossary of Thesis Terms . . . . . . . . . . . . . . . . . 11

1.2 Acronyms used in Thesis . . . . . . . . . . . . . . . . 12

3.1 Parameter choices for velocity recovery from synthetics . . . . . . . 43

A.1 Seismic Station Information _. . . . . . . . . . . . . . 132

A.2 Station-pair dataset preprocessing parameters . . . . . . . . . . . 134

A.3 Station-pair dataset filter table (MSNoise Version 1.4) . . . . . . . . 135

A.4 Customised parameters for station-pair velocity analysis . . . . . . . 135

A.5 Preprocessing Parameters for Single-Station Dataset (MSNoise Version 1.5.1) . . . . . . . . . . . . . . . . . . . 136

A.6 White Island dataset 0.1-1.0 Hz Filter Table (MSNoise Version 1.5.1)136

A.7 White Island dataset 1.0-2.0 Hz Filter Table (MSNoise Version 1.5.1)137 


\section{Chapter 1}

\section{Introduction}

This chapter covers the primary motivations and objectives of this thesis (Section 1.1 and 1.2), setting the scene for the remaining chapters. We introduce White Island volcano and outline the recent activity driving this study (Section 1.3). Finally, we describe the intended experimental design and the thesis layout (Section 1.4 and $1.5)$.

\subsection{Motivation}

A fundamental objective of volcanology is to determine where and when volcanic eruptions will occur. Volcanic activity can be considered the end result of ascending magma interacting with the Earth's surface. Detecting rising magma, or the disturbance of a pre-existing shallow chamber, is crucial for determining when an eruption will occur. Increases in sub-surface pressures in the build up to volcanic eruptions are expected to induce crustal deformation (Sparks, 2003; Patanè et al., 2003; Brenguier et al., 2008b). Thus, a wide variety of disciplines have focussed on monitoring pre-cursory activity at volcanoes in an attempt to understand volcanic processes (Sparks, 2003; Dzurisin, 2003). In the geophysical domain, common approaches include recording seismicity, measuring ground deformation - for example by using the Global Positioning System (GPS) or Synthetic Aperture Radar (SAR) - and monitoring volcanic gas flux (Sparks, 2003; Dzurisin, 2003). Each has its limitations however, and it is becoming clear that the integration of cross-disciplinary data is crucial for successful forecasting (Sparks, 2003).

Recent advances in seismology have opened an exciting new doorway for monitoring small crustal perturbations using seismic ambient noise. This typically refers to seismic energy generated through the interaction of ocean waves with the Earth's surface (discussed further in Chapter 2). Significantly, ambient noise can be used to produce continuous seismic velocity measurements capable of detecting small 
changes in the elastic properties of the crust. This enables information to be gathered in the absense of seismicity, for example during aseismic magma pressurization or migration (Brenguier et al., 2008b). Further, unlike GPS and SAR monitoring, the detection of crustal changes is not limited to perturbations that affect only the Earth's surface. The technique has therefore become increasingly popular for monitoring volcanoes, with the number of studies rapidly growing. Further development is required however to unlock its full potential.

White Island volcano, in New Zealand, represents an excellent case study for ambient noise based monitoring. Not only have there been multiple well-documented eruptions within the last six years, but these followed a decade of relative inactivity. This presents an opportunity to study the volcano at different stages during its eruptive cycle. Furthermore, White Island has been - and continues to be studied by a broad range of disciplines. Thus, there are prospects for comparing ambient noise based observations at the volcano with those from the wider scientific community.

\subsection{Primary Objectives}

The key objectives for this study are divided into three separate points of inquiry. These should be considered in the context of the long-term goal to understand the eruptive life cycle of volcanoes and the potential of ambient noise based monitoring.

1. Investigate seismic velocity changes at White Island volcano and their connection to volcanic activity. What can they tell us about dynamic changes occurring beneath the volcano? Do the results support or, alternatively, shed new light on previous observations at White Island?

2. Investigate alternative sources of velocity changes that are linked to nonvolcanic processes. Additionally, consider how these might influence interpretations if not accounted for.

3. Consider the implications for real-time ambient noise monitoring and possible limitations associated with eruption forecasting.

\subsection{White Island Volcano}

White Island, also known by the Māori name Whakaari, has been New Zealand's most active volcano in historical times (Cole et al., 2000). It is located approximately $50 \mathrm{~km}$ offshore of the Bay of Plenty coast (Fig. 1.1) and, in historical times, has been 
characterised by intermittent eruptive episodes (Cole et al., 2000). These generally do not pose a threat to onshore New Zealand (Johnston et al., 1999) though may present a local hazard for the $>13,500$ annual visitors (Letham-Brake, 2013). In this section, the geological setting of White Island is described in addition to the recent activity motivating this study.

\subsubsection{Geological Setting}

New Zealand volcanism is controlled by the oblique subduction of the Pacific Plate underneath the Australian Plate (Fig. 1.1) (Wright, 1992; Cole et al., 2000). The youngest expression of this volcanism is found in the Taupo Volcanic Zone, the southernmost portion of the Tonga-Kermadec arc system (Cole and Lewis, 1981). It represents a region of backarc extension extending from the Havre Trough into the central North Island (Stern, 1987; Wright, 1992), albeit offset sinistrally by 40 $50 \mathrm{~km}$ (Wright, 1992). The eastern side of the Taupo Volcanic Zone coincides with an active, roughly linear, alignment of andesitic volcanoes extending from Ruapehu to the Whakatane seamount. In the central Taupo Volcanic Zone, however, rhyolitic volcanism dominates (Cole et al., 2000). Volcanoes therefore represent a significant hazard to people living in the North Island of New Zealand. 


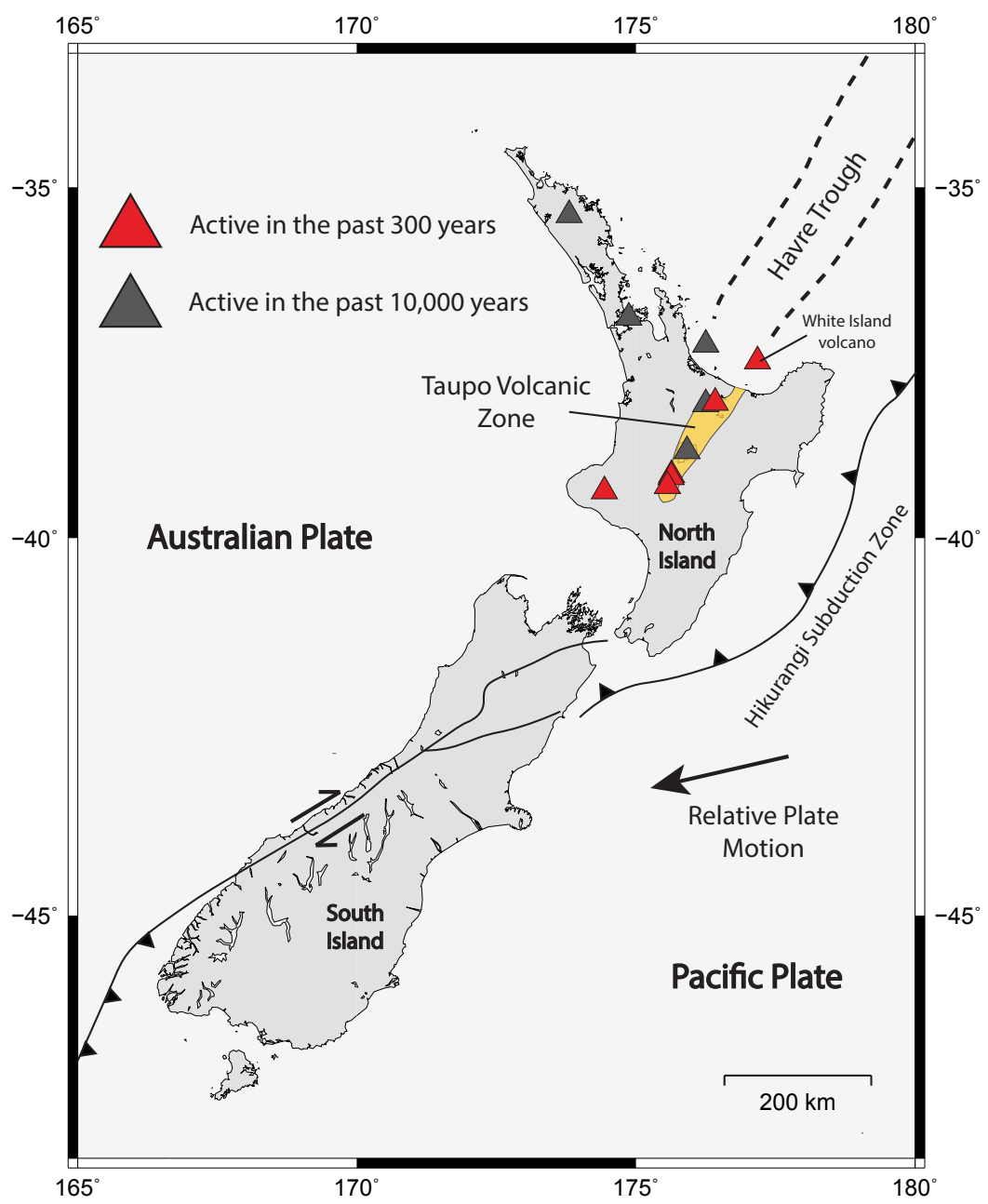

Figure 1.1: Geological setting of New Zealand volcanism, showing the location of volcanoes active within the past 300 and 10,000 years, following Becker et al. (2010).

White Island is an active stratovolcano that marks the southern end of the TongaKermadec seamount chain and the northernmost point of the Taupo Volcanic Zone (Cole and Lewis, 1981). The island itself is the subaerial expression of a larger submarine volcano that rises 300-400 m from the sea floor covering an area $16 \mathrm{~km}$ $\times 18 \mathrm{~km}$ (Duncan, 1970). Above the surface, the island measures $2.0 \times 2.4 \mathrm{~km}$ with a summit $321 \mathrm{~m}$ above sea level (Fig. 1.2). Its surface is dominated by two overlapping cones, an older eroded western cone (Ngatoro) and a younger Central Cone (Black, 1970; Duncan, 1970). Each of these is filled with andesite-dacite lava flows, breccias, agglomerates, and unconsilidated beds of ash and tuffs containing lava blocks (Black, 1970; Cole et al., 2000). The Main Crater, which occupies a $0.4 \times 1.6 \mathrm{~km}$ area of the central cone, is further divided into the western, eastern and northern subcraters (Houghton and Nairn, 1989). All recent volcanic activity and outgassing has been confined to the western subcrater (Werner et al., 2008; Bloomberg et al., 2014), which is currently occupied by a crater lake. 
White Island hosts a large volcano-hydrothermal system within the upper kilometre beneath the Main Crater (Giggenbach and Glasby, 1977; Nishi et al., 1996). Surface expressions of this system include fumaroles, hot springs, mud pools and an acidic crater lake. Eruptions at the volcano are predominantly phreatic, phreatomagmatic, and strombolian in style, typically emitting only small volumes of eruptive products (Houghton and Nairn, 1991; Cole et al., 2000; Peltier et al., 2009). The frequency of weak eruptive events suggests a long-term passive interaction of magma with the hydrothermal system (Houghton and Nairn, 1991). Discrete larger events also occur, with significant magmatic episodes occurring between 1976-1982 and 1986-1992 associated with major episodes of lava extrusion (Houghton and Nairn, 1989; Cole et al., 2000). Magmas feeding these eruptions are thought to originate from magma chambers as shallow as $500 \mathrm{~m}$, with evidence of deeper chambers located between 1-2 and 2-7 km depth beneath the surface (Houghton and Nairn, 1989; Cole et al., 2000).

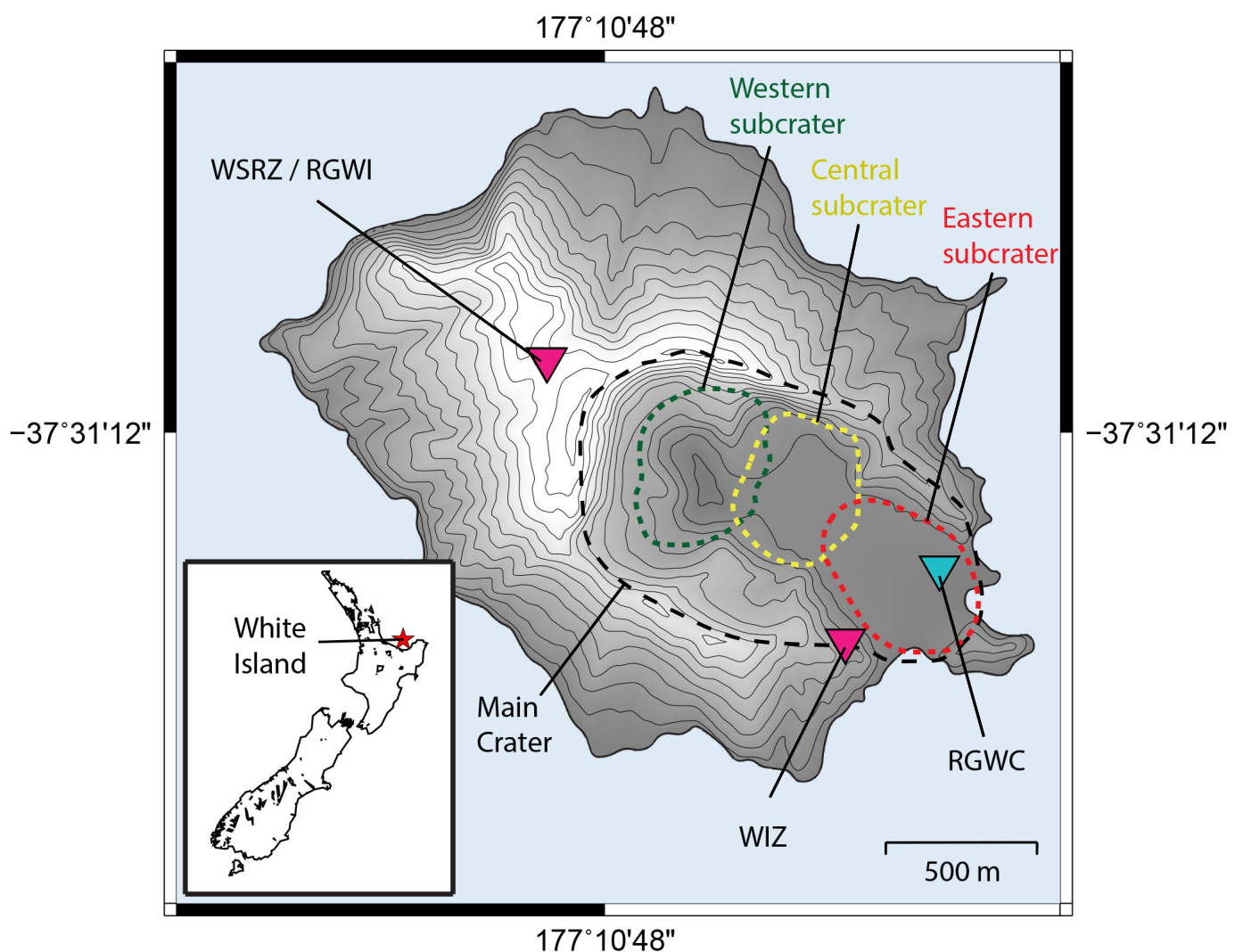

Figure 1.2: Map of White Island volcano. The position of permanent seismic stations WIZ and WSRZ (upside-down purple triangles) and continuous GPS sites RGWI and RGWC (upside-down blue triangles) are shown. Note that sites RGWI and WSRZ are not distinguishable at the resolution shown. Contours represent 25 meter intervals. Craters and subcraters are drawn following Moon et al. (2005). 


\subsubsection{Recent Eruptive History}

Prior to 2007, when our dataset begins, the most recent volcanic activity occurred in the year 2000. This was the largest magmatic eruption of a highly active period stretching back nearly 40 years, with significant volcanic events occuring every few years (Peltier et al., 2009). Following the 2000 eruption however, White Island remained in a low state of unrest for over ten years - a long time relative to previous decades. Then in August 2012, the volcano experienced its first eruption in 12 years, signalling the start of a new phase of eruptive activity. This section describes eruptive activity from 2012-2016, with key events summarised in Figure 1.3.

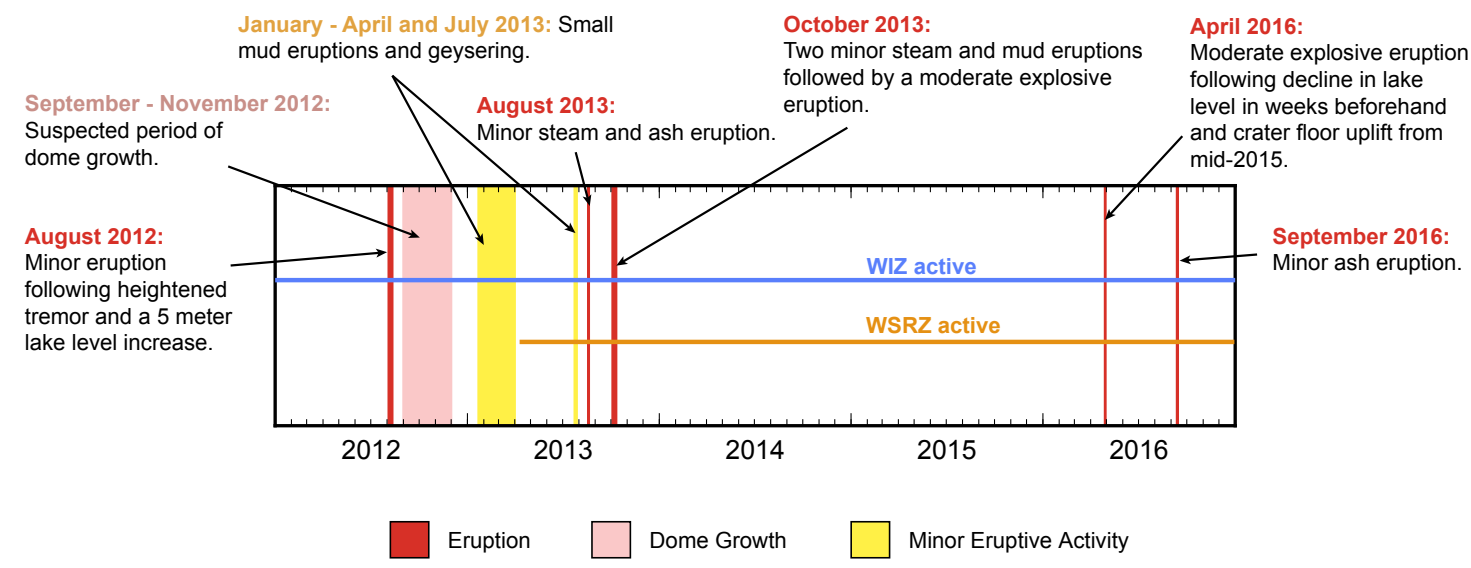

Figure 1.3: Timeline of eruptive activity at White Island volcano between 2012-2016, with the dates WIZ and WSRZ stations are active shown. Years 2007-2011 are not shown as no significant volcanic activity occurred within this period.

First signs of unrest leading up to the eruption in 2012 were recorded as early as August 2011. Analysis from a 6 month temporary seismic deployment during this time found significant seismicity consisting of very long-period, long-period and high frequency earthquakes (Jolly et al., 2017). Elevated levels of tremor followed, coinciding with declines of the crater lake level (Chardot et al., 2015). By May 2012, increased $\mathrm{SO}_{2}$ emissions had been recorded (Chardot et al., 2015). Two significant episodes of tremor occurred in the final two months before the eruption, with the later of these, recorded on 27-28 July 2012, occurring only hours before a 5 metre increase in the lake level (Chardot et al., 2015). Intermittent tremor continued for the next few days, finally ending with the 5th August 2012 eruption (Chardot et al., 2015).

Between August 2012 and October 2013 White Island experienced five ash and steam eruptions, numerous small mud and ash eruptions, and extruded a small lava dome (Chardot et al., 2015; Jolly et al., 2016). The 5th August 2012 eruption ejected 
ash and blocks onto the crater floor, with elevated tremor and ash venting occuring in the days following (Chardot et al., 2015). This evolved to small mud eruptions by the end of August (Chardot et al., 2015). Volcanic tremor remained elevated between September and December 2012, though with less variation than in August (Chardot et al., 2015). During this period, a small lava dome - first observed in November - developed on the island (Chardot et al., 2015). The exact timing of this is unclear, though is thought to have been between September and November 2013 (Chardot et al., 2015; Jolly et al., 2017). By January 2013, activity around Crater Lake had become more intense, characterised by small mud eruptions that continued intermittently through to early April (Chardot et al., 2015; Jolly et al., 2016). This coincided with the drying out of the crater lake, which was later reestablished following increased rainfall late-April (Chardot et al., 2015; Christenson et al., 2017). Minor activity at the volcano resumed in July with further geysering, declining by early August (Chardot, 2015). A small eruption then occurred on 20 August 2013, mainly producing steam with a small proportion of volcanic ash (Chardot, 2015). October 2013 produced further short-lived eruptions, with a steam venting event on 4 October and a minor steam and mud eruption on 8 October (Chardot et al., 2015). Finally, the volcano experienced a moderate explosive eruption on 11 October, depositing material over the whole crater floor (Chardot et al., 2015). This marked the end of a significantly active period at White Island.

Activity resumed in late April 2016 with a moderate phreatic eruption, followed by an eruption in mid-September of the same year. The first of these was preceded by heightened volcanic tremor and a lake level decrease in the weeks prior (Hamling, 2017). Synthetic aperture radar observations indicate that the April 2016 eruption was preceded by uplift of the crater floor from as early as mid-2015 (Hamling, 2017). Subsequent field observations following the eruption revealed that it had emptied the crater lake, generating a surge deposit that covered much of the crater floor (Hamling, 2017). Significant collapse had also occurred along the crater edge during the eruption in addition to post-eruption landsliding (Hamling, 2017). Activity remained low from May 2016, until a minor ash eruption occurred in September. This is thought to be the result of loosened material being ejected, with both gas and seismicity remaining at low levels (Hamling, 2017). As of mid-2018, no further eruptive activity has occurred.

\subsection{Study Design}

Monitoring of White Island is limited by having only two permanent seismic stations on the volcano (Fig. 1.2). Further, only station WIZ has been operating throughout 
the study period, with station WSRZ becoming operational only in mid-2013 (see Appendix A.1 for station details). It is therefore an interesting setting for volcano monitoring using ambient noise, where previous studies have been afforded greater seismic coverage (e.g. Brenguier et al., 2008b; Bennington et al., 2015; Donaldson et al., 2017). This complicates the survey design at White Island, where ambient noise work usually monitors seismic energy travelling between pairs of stations. Fortunately, recent work has shown similar results can be obtained at volcanoes by using a single seismic station, cross-correlating between the different components (De Plaen et al., 2016). This approach is clearly well suited to White Island, and represents an opportunity to test the feasibility of monitoring when seismic coverage is sparse.

This study applies two survey designs towards monitoring White Island volcano. The first approach involves using pairs of seismic stations that begin onshore and end at one of the permanent stations on the volcano (Chapter 3). This follows the more conventional approach to ambient noise monitoring, albeit with much larger interstation distances than usual at volcanoes. The second approach involves processing single seismic stations individually (Chapter 4), following the recent work of De Plaen et al. (2016). Both survey designs represent relatively untested approaches to monitoring a volcano. Thus, we consider this an interesting opportunity to consider different ways of monitoring volcanoes that are located offshore.

\subsection{Thesis Layout}

This section includes general notes that the reader should know before continuing (Section 1.5.1), a summary of the thesis structure (Section 1.5.2), and a glossary of terms and acronyms used throughout the thesis (Section 1.5.3).

\subsubsection{General Notes}

- Volcanic activity is assigned into three catagories for simplified visual presentation: (1) eruptions, (2) dome growth, and (3) minor eruptive activity. The plot style, and corresponding legend, is demonstrated in Figure 1.3 and is used throughout this thesis.

- This thesis uses 'we' instead of 'I' throughout. This is a personal preference and should not be used to determine the level of external input into the described content. Any data that were not processed by myself, in addition to sources of external feedback outside of thesis supervisors, is declared in the Chapter Outline (Section 1.5.2). 
- Content included in the Appendices is not essential for communicating the methodology and outcomes of the thesis and therefore represents optional reading.

\subsubsection{Chapter Summary}

Chapter 2 - Background Theory: This chapter describes the background theory required to understand how seismic velocity changes are measured from ambient noise. This includes a description of what is meant by ambient noise interferometry (Section 2.1) and a brief review of application to volcanoes globally (Section 2.2). Finally, the underlying software used to process noise records through to velocity changes is described (Section 2.3).

Chapter 3 - Station-pair Dataset: This chapter describes the processing for ambient noise recorded using pairs of stations that include of a seismic station onshore and a station offshore on White Island. Considerable parameter testing is performed in Section 3.2 that serves as a platform for similar processing in Chapter 4. The chapter concludes by presenting velocity changes recorded with this dataset (Section 3.3). An early version of Section 3.2 was reviewed by Corentin Caudron.

Chapter 4 - Single-Station Dataset: This chapter describes the processing for single-station ambient noise work. Both permanent stations on White Island are processed in addition to four stations onshore. Steps involved in justifying parameters (Section 4.2) follow those of Chapter 3. The chapter concludes by presenting velocity changes recorded by White Island stations (Section 4.3.1), comparing these to velocity changes recorded by onshore single stations (Section 4.3.2) and the timing of nearby tectonic earthquakes (Section 4.3.3).

Chapter 5 - Discussion: This chapter discusses the results of Chapters 3 and 4 in the context of identifying whether observed changes are volcanic or non-volcanic in their origin. First, we examine the depth sensitivity of velocity changes recorded by each dataset and consider whether velocity changes recorded by station-pairs are occurring in the vicinity of the volcano given large station separation distances (Section 5.1). Non-volcanic processes, such as meteorological influences and tectonic earthquakes, are then considered as sources of background velocity changes (Section 5.2) This sets the platform to discriminate between volcanic and non-volcanic processes influencing seismic velocities at White Island (Section 5.3). The chapter concludes by discussing the possibilities of real-time monitoring at the volcano (Section 5.3.3). An early version of Section 5.2.1 was reviewed by Corentin Caudron and RSAM data in Section 5.3.1 provided by Art Jolly. 
Chapter 6 - Conclusions: This chapter summarises the key findings of this thesis in the context of the original primary objectives in Section 1.2. Additionally, we reflect on the suitability of station-pair and single-station datasets towards recording velocity changes at the volcano.

Appendices: The appendices are split into two parts. The first part contains supplementary material relating to data processing (Appendix A), including station information and final processing parameters. The second part includes velocity changes that have been excluded from the main body of the thesis (Appendix B). Information within the appendix is referred to in text where it is relevant.

\subsubsection{Glossary of Terms and Acronyms}

This section provides a brief description of terms used throughout this thesis (Table 1.1) and a list of acronyms and their meanings (Table 1.2). These are not necessarily applicable outside of this thesis.

When defining a station-pair and component-pair used in the cross-correlation of two seismic stations, e.g. components RT for station-pair OPRZ-WIZ, the first component listed corresponds to the first station listed. In the example provided then, this would be a cross-correlation between the radial component at OPRZ station and the transverse component at WIZ station. In the resultant cross-correlation function, positive lag times reflect energy travelling from the first station to the second station while negative lag times reflect energy travelling from the second station to the first station. Thus, using the same example, positive lags would reflect energy travelling from OPRZ to WIZ and negative lags energy travelling from WIZ to OPRZ station. 
Table 1.1: Glossary of Terms used in thesis

\begin{tabular}{|c|l|}
\hline Term & Description \\
\hline Auto-correlation & $\begin{array}{l}\text { Cross-correlation of time series with itself. Here, this refers to cross- } \\
\text { correlating components EE, NN or ZZ during single-station processing. }\end{array}$ \\
\hline Component-pair & $\begin{array}{l}\text { Refers to the seismometer components that have been cross-correlated. } \\
\text { For example, component-pair ZZ is a cross-correlation between two ver- } \\
\text { tical components. }\end{array}$ \\
\hline Cross-component & $\begin{array}{l}\text { Cross-correlation of the components of a single seismic station, excluding } \\
\text { auto-correlations. Here, this refers to cross-correlating components EN, } \\
\text { EZ or NZ during single-station processing. }\end{array}$ \\
\hline Cross-correlation & $\begin{array}{l}\text { A measure of similarity between two time series as a function of relative } \\
\text { displacement (denoted the lag time). }\end{array}$ \\
\hline function & CCF to be compared with the reference stack during MWCS technique. \\
\hline Lag time & $\begin{array}{l}\text { Measure of relative time domain offset between two time series in cross- } \\
\text { correlation function. }\end{array}$ \\
\hline Noise pathway & The line connecting two stations used for ambient noise interferometry. \\
\hline Offshore station & Seismic station located off mainland New Zealand (e.g. White Island). \\
\hline Onshore station & Seismic station located on mainland New Zealand. \\
\hline Reference stack & $\begin{array}{l}\text { Background CCF to be compared with current stacks during MWCS } \\
\text { technique. }\end{array}$ \\
\hline Single-station & Denotes a single seismometer cross-correlated with itself. \\
\hline Station-pair & Denotes two seismometers that are cross-correlated with eachother. \\
\hline
\end{tabular}


Table 1.2: Acronyms used in thesis

\begin{tabular}{|c|c|}
\hline Acronym & Definition \\
\hline $\mathrm{AC}$ & Autocorrelation \\
\hline $\mathrm{CC}$ & Pearson's correlation coefficient \\
\hline $\mathrm{CCF}$ & Cross-correlation function \\
\hline $\mathrm{EE}$ & East-east component \\
\hline $\mathrm{EN}$ & East-north component \\
\hline EZ & East-vertical component \\
\hline FDSN & $\begin{array}{l}\text { International Federation of Digital } \\
\text { Seismograph Networks }\end{array}$ \\
\hline FFT & Fast Fourier transform \\
\hline GPS & Global positioning system \\
\hline LHS & Left hand side (referring to equation) \\
\hline$M_{W}$ & Moment magnitude \\
\hline MWCS & Moving-window cross-spectral \\
\hline NN & North-north component \\
\hline NZ & North-vertical component \\
\hline RHS & Right hand side (referring to equation) \\
\hline RMS & Root-Mean-Square \\
\hline $\mathrm{RR}$ & Radial-radial component \\
\hline RSAM & Real-time seismic amplitude measurement \\
\hline $\mathrm{RT}$ & Radial-transverse component \\
\hline $\mathrm{RZ}$ & Radial-vertical component \\
\hline SAR & Synthetic Aperture Radar \\
\hline $\mathrm{SC}$ & Single-station cross component \\
\hline $\mathrm{SE}$ & Standard Error \\
\hline SNR & Signal-to-Noise Ratio \\
\hline $\mathrm{TR}$ & Transverse-radial component \\
\hline $\mathrm{TT}$ & Transverse-transverse component \\
\hline $\mathrm{TZ}$ & Transverse-vertical component \\
\hline $\mathrm{ZR}$ & Vertical-radial component \\
\hline $\mathrm{ZT}$ & Vertical-transverse component \\
\hline $\mathrm{ZZ}$ & Vertical-vertical component \\
\hline
\end{tabular}




\section{Chapter 2}

\section{Background Theory}

The objective of this chapter is to cover the background theory that underpins this thesis. The first section addresses the ambient noise wavefield, what it is and how recent developments have allowed us to use it as a tool for monitoring the subsurface (Section 2.1). Discussion then moves towards application to volcano monitoring (Section 2.2), with examples of similar studies described. Finally, the Python package MSNoise (Lecocq et al., 2014) is introduced, with a review of the different processing options available (Section 2.3). This sets the platform for greater scrutiny of these choices in Chapter 3.

\subsection{Ambient Noise Interferometry}

In the past, seismic energy recorded by seismometers has been focussed on the detection of phenomena such as earthquakes or explosions. Consequently, alternative sources of background energy were typically supressed. Recent developments, however, have enabled seismic background noise, otherwise known as ambient noise, to be extracted for the purpose of sampling sub-surface velocities (Shapiro and Campillo, 2004; Shapiro et al., 2005). In doing so, a new avenue of exploration and monitoring opened, with the number of studies now using this approach rapidly expanding. Here, the general properties of the ambient noise wavefield are examined in addition to the key developments that led to ambient noise interferometry as it is today.

\subsubsection{Wavefield Properties}

In the nineteeth century it was discovered that the Earth's surface undergoes very small movements unrelated to earthquake or explosive activity (Gutenberg, 1958). These were first observed by sensitive instruments during nonseismic research; for example, while undertaking gravity surveys (Gutenberg, 1958). The introduction of 
more sensitive seismometers in the twentieth century came with the understanding that these movements were not intermittent; rather the Earth's surface was in a state of continuous motion (Gutenberg, 1951). Such motion is driven by energy originating from a variety of sources, distinguishable through their statistical and spectral properties. Human activity, for example, typically produces sporadic seismic energy at periods below one second and is generally referred to as artificial noise (Gutenberg, 1958). Another kind of noise that dominates the spectrum, at periods between approximately 5-15 seconds, arises from the interaction of ocean waves with the surface. These are known as oceanic microseisms, and make up the majority of the noise wavefield. Thus, seismometers are noisier the closer they are to the coastlines (Stein and Wysession, 2003). At even longer periods, the Earth's free oscillations are observed, also known as Earth 'hum' (Nawa et al., 1998; Suda et al., 1998). These oscillations are considered to be excited by oceanic infragravity waves interacting with the seafloor topography (Rhie and Romanowicz, 2004) and atmospheric forcing (Kobayashi and Nishida, 1998).

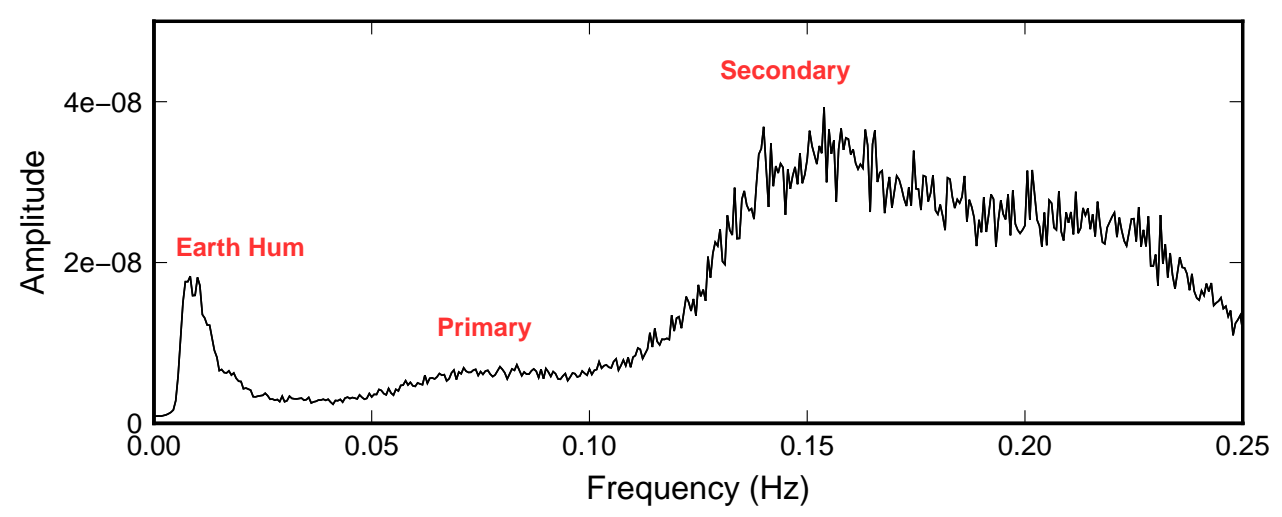

Figure 2.1: Median noise spectra over 100 days at WIZ station in 2014. For each day, a Fast Fourier Transform is performed on a 30 minute window of raw noise and bandpassed between 0.07 and $0.25 \mathrm{~Hz}$. The primary microseism is visible at approximately $0.07 \mathrm{~Hz}$ and the second at approximately $0.15 \mathrm{~Hz}$. Earth Hum is visible at lower frequencies.

Ambient noise studies typically use energy originating from oceanic microseisms, due to strong temporal stability and their ability to travel great distances. Oceanic microseisms are dominated by two spectral peaks at approximately 7 and 14 second periods (e.g. Peterson, 1993; Stutzmann et al., 2000). These are commonly referred to as the primary (14 second peak) and secondary (7 second peak) microseisms. Primary microseisms are generated through the interaction of ocean waves with the coastal seafloor (Hasselmann, 1963) and consist of both Love and Rayleigh waves (Friedrich et al., 1998). The energy ratio between these is approximately 1.2 in favour of Love waves (Friedrich et al., 1998). Secondary microseisms, on the other 
hand, are produced through the non-linear interference of incoming and reflected ocean waves (Longuet-Higgins, 1950). This interaction results in the generation of a standing wave which, because of minimal attenuation with depth, can excite surface waves on the ocean floor (Longuet-Higgins, 1950; Hasselmann, 1963). Unlike primary microseisms, secondary microseisms consist predominantly of Rayleigh waves (Haubrich and McCamy, 1969; Friedrich et al., 1998) and are stronger in amplitude. This is clearly observed at White Island, where the secondary microseism dominates the noise spectra over a one-hundred day interval (Fig. 2.1).

\subsubsection{Green's Function Recovery}

Seismic interferometry refers to the principle of cross-correlating seismic responses recorded at different receivers to obtain an approximate impulse response between them (Wapenaar et al., 2010). The impulse response is known as the Green's Function, and represents the seismic response that would be measured at each receiver if the source were located at the other (Fig. 2.2). Early developments in seismic interferometry originate with Aki (1957), who was the first to demonstrate that seismic noise could be used to extract information on the propagation media. Claerbout (1968) later demonstrated that the impulse response of a medium could be approximated through the autocorrelation of signals at the surface. The first applications of ambient noise cross-correlations had to wait until the end of the twentieth century, in the field of helioseismology. Duvall et al. (1993) used cross-correlations of dopplergram traces to measure the travel times of acoustic waves, overcoming the random nature of solar seismic sources. Moving into the twenty-first century, Weaver and Lobkis (2001) and Lobkis and Weaver (2001) demonstrated that the Green's function could be obtained from cross-correlating diffuse wavefields in laboratory conditions. These key contributions to our understanding of random wavefields, and their practical uses in recovering surface wave information, paved the way for modern usage of ambient noise.

The key breakthrough in ambient noise interferometry came with the demonstration that a good approximation to the seismic Green's function can be obtained by cross-correlating ambient noise recorded at pairs of seismometers (Shapiro and Campillo, 2004; Sabra et al., 2005; Shapiro et al., 2005). This removes the reliance on discrete seismic sources, as required by active source or coda wave interferometry (e.g. Snieder et al., 2002; Campillo and Paul, 2003). Additionally, the Green's function has been found to be recoverable over a wide range of length scales, from a few kilometers between seismic stations (e.g. Sabra et al., 2005; Brenguier et al., 2007) to thousands of kilometers (e.g. Yang et al., 2007; Bensen et al., 2008). Thus, the method has become a favourable tool globally for seismic tomography (e.g. Yao 
(a)

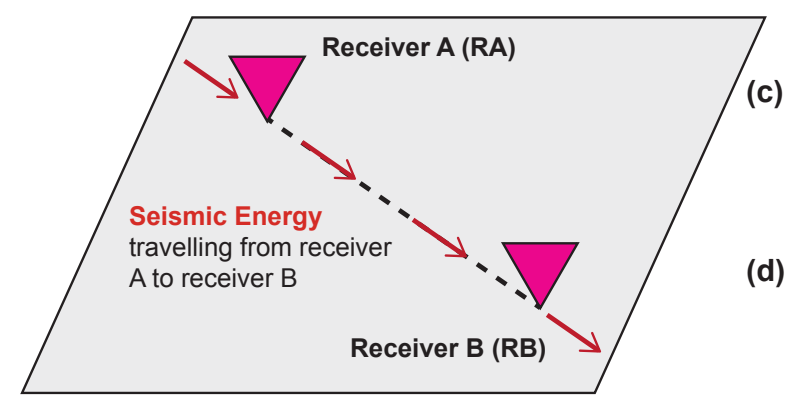

(b) RA

(c) RB
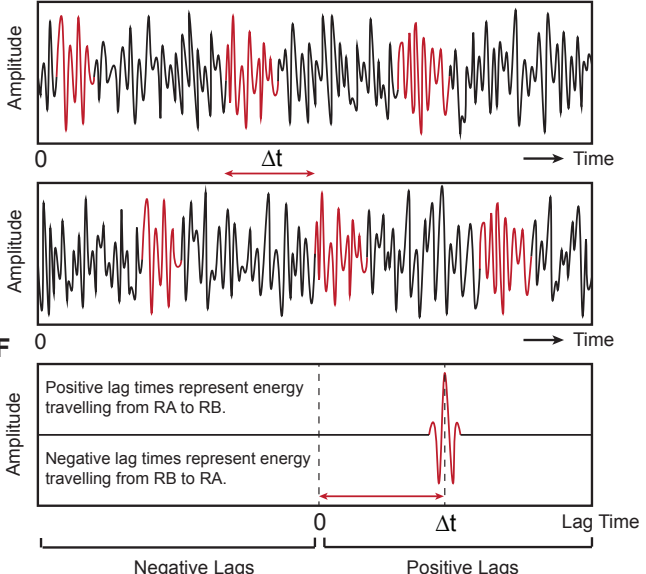

Figure 2.2: Demonstration of the cross-correlation of seismic energy travelling between pairs of receivers. (a) Seismic energy travels on a direct path between receivers A and B. (b) Noise recorded by receiver A. (c) Noise recorded by receiver B. Coherent energy that has travelled between the two receivers is shown in red. (d) Crosscorrelating the two time series ( $R A$ and $R B$ ) recovers the travel time of coherent energy in the form of a cross-correlation function (CCF).

et al., 2006; Cho et al., 2007; Yang et al., 2007; Lin et al., 2007; Behr et al., 2010; Godfrey et al., 2017).

Recovery of the Green's function assumes that noise sources are equipartioned, that is, uniformly distributed with a zero net flux (Snieder, 2004; Sánchez-Sesma and Campillo, 2006). In reality this is rarely the case, with tendencies for azimuthal biases in noise strength and limited independent sources (Tsai, 2010). The question is then whether recovery of the Green's function is possible in real-world conditions. Wapenaar (2006) demonstrated that a one-sided distribution of noise sources can accurately resolve the Green's Function on one side of the cross-correlation function. Additionally, Snieder (2004) argued that the assumption of equipartitioned noise souces can be relaxed in place of an assumption that scattered waves propagate isotropically near receivers. Finally, Tsai (2010) demonstrated that assumptions of equipartition are not required under specific conditions, dependent on the degeneracy of normal modes that describe the noise wavefield. Thus, it has been shown that approximations of the Green's function can still be recovered without satisfying all theoretical assumptions.

Sufficiently long records of ambient noise are typically required for accurate recovery of the Green's Function. This is not a problem for tomography based work, which typically uses noise records on the order of years (Hadziioannou et al., 2009). However, for monitoring temporal velocity changes, smaller noise records are required 
in order to repeatedly compute cross-correlation functions using noise recorded at different times. This allows temporal information on the medium to be gathered, discussed further in Section 2.1.3. Fortunately, it has been demonstrated that full reconstruction of the Green's Function is not required for monitoring small changes (Hadziioannou et al., 2009). Rather, the stability of noise sources is the most important condition for reducing errors in measurements (Hadziioannou et al., 2009). Thus, the assumptions required for noise-based seismic imaging can be further relaxed for monitoring purposes.

Finally, it is possible to compute ambient noise cross-correlations using seismic energy recorded at only a single-station (e.g. Wegler and Sens-Schönfelder, 2007; Minato et al., 2012; Hobiger et al., 2014; De Plaen et al., 2016). Instead of seismic energy travelling between pairs of stations, single-station ambient noise relies on recording back-scattered energy i.e. energy that is recorded by the receiver more than once. Monitoring is then possible by either correlating the station components with themselves to give auto-correlations (AC) or performing single-station crosscomponent (SC) correlations, where the different components are correlated with each other. Using a single seismic station then, it is possible to obtain six independent two-sided cross-correlation functions using autocorrelations (EE, NN, ZZ) and cross-components (EN, EZ, NZ).

\subsubsection{Measuring Seismic Velocity Changes}

Measuring temporal seismic velocity changes is a further useful application of ambient noise interferometry. This takes advantage of the sensitivity of waves travelling along the Earth's surface to small perturbations in the elastic properties of the crust (e.g. Aki, 1957; Sato et al., 2012). This is easily demonstrated by examining the relationship between stress and strain in a linearly elastic material:

$$
\sigma_{i j}=c_{i j k l} \epsilon_{k l}
$$

where $\sigma_{i j}$ represents the stress tensor and $\epsilon_{k l}$ the strain tensor (Stein and Wysession, 2003). Constants $c_{i j k l}$, known as the elastic moduli, describe the properties of the material via 36 independent components. A useful approximation is to assume material within the Earth is isotropic, reducing the number of independent components to two elastic moduli, $\lambda$ and $\mu$. These are known as the Lamé constants. Compressional $(\alpha)$ and shear wave $(\beta)$ speeds can then be written in terms of these moduli as:

$$
\alpha=\sqrt{\frac{\lambda+2 \mu}{\rho}}, \quad \beta=\sqrt{\frac{\mu}{\rho}}
$$


where $\rho$ is density of the material the waves pass through. It is clear then that changes in the elastic moduli, assuming a constant density, induce changes in the seismic velocity.

Changes in seismic velocity can be measured following the methodology introduced by Poupinet et al. (1984). Using earthquake doublets, they recorded relative velocity perturbations by measuring the time delay between individually recorded microearthquakes within a series of moving-windows. The fractional change in shear velocity was then found by examining the time delay as a function of time on the corresponding seismograms, assuming that any resultant velocity change is distributed homogeneously over the sampled region. Under this approximation, the new arrival time of seismic energy — assuming the same source location — is given by:

$$
t+\delta t=\frac{d}{v+\delta v}
$$

where $d$ is the distance travelled, $t$ is the original arrival time, $\delta t$ the time delay, $v$ the original velocity and $\delta v$ the change in velocity. Rearranging for $d$ and multiplying out the terms gives:

$$
d=v t+v \delta t+t \delta v+\delta v \delta t
$$

If it is assumed that measured velocity changes are small, i.e. $\delta v \delta t \approx 0$, then this can be rearranged to:

$$
\frac{\delta t}{t}=-\frac{\delta v}{v}
$$

Thus the fractional change in velocity can be found by taking the slope of measured time delays against the seismogram run time.

The doublet technique, as it is known, can be similarly applied to cross-correlations of ambient noise to track velocity changes through time (e.g. Brenguier et al., 2008b). The basic idea is to compare cross-correlation functions produced using smaller subsets of recorded noise (defined as current functions) with a reference cross-correlation function generated using a larger noise record (Fig. 2.3). Changes in the arrival time of energy travelling between seismic stations, compared to the reference function, are interpreted to reflect a change in velocity using Equation 2.5 (Fig. 2.3c). Typically this focusses on later arrivals in the cross-correlation function, corresponding 
(a)

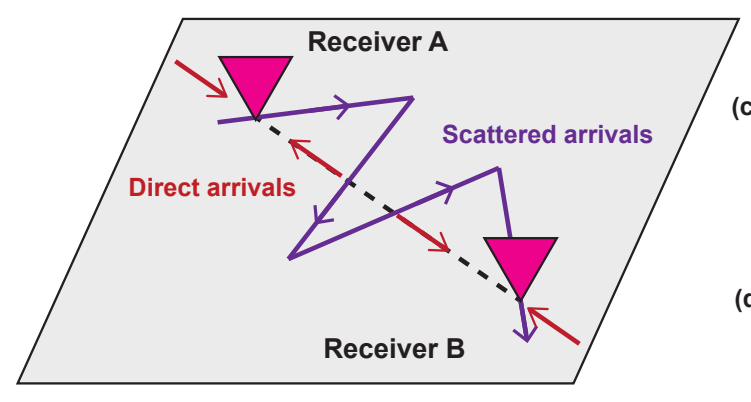

(b) Reference CCF

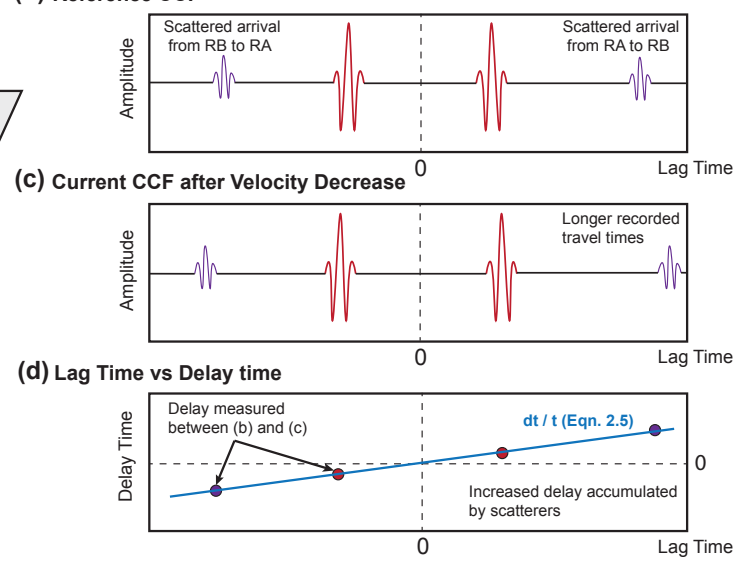

Figure 2.3: Demonstration of how velocity changes are recovered from ambient noise CCFs. (a) Direct and scattered energy travels between receivers. (b) Reference CCF with peaks showing the travel times for direct and scattered arrivals. (c) Current CCF after a decrease in seismic velocity. (d) Delay times are measured for direct and scattered arrivals between the current and reference CCF. Linear slope is fitted for the LHS of Equation 2.5. The positive slope represents a velocity decrease.

to scattered energy, which spend longer sampling the subsurface than direct ballistic arrivals. Consequently, scattered arrivals accumulate larger delay-times (Fig. 2.3c), improving the sensitivity of the measurements to small changes. Furthermore, scattered arrivals are less sensitive to temporal changes in the noise source distribution relative to direct arrivals (Colombi et al., 2014). Thus, using scattered arrivals for measuring velocity changes is preferred. We discuss specific application of the doublet technique in this thesis in Section 2.3.

The number of studies now using ambient noise for monitoring purposes is rapidly growing, with many natural phenomena found to produce measurable seismic velocity changes. These include short and long-term volcano related deformation (e.g. Brenguier et al., 2008b; Obermann et al., 2013; Rivet et al., 2014), co- and postseismic deformation following tectonic earthquakes (e.g. Brenguier et al., 2008a; Chen et al., 2010; Minato et al., 2012; Froment et al., 2013; Taira et al., 2015; Heckels et al., 2018), precipitation (e.g. Sens-Schönfelder and Wegler, 2006; Hillers et al., 2014), atmospheric pressure loading (e.g. Silver et al., 2007; Niu et al., 2008), atmospheric temperature induced thermoelasticity (e.g. Meier et al., 2010; Richter et al., 2014; Hillers et al., 2015) and tidal cycles (e.g. Yamamura et al., 2003; Takano et al., 2014). The magnitude of these velocity changes tends to be on the order of $0.1 \%$. With so many possible sources of change, it is crucial that we improve our understanding of the relative influence of these processes. At volcanoes, this will improve our ability to distinguish between volcanic and non-volcanic sources. 


\subsection{Monitoring Volcanoes with Ambient Noise}

A wide variety of different approaches have now been applied detect crustal changes around volcanoes. These include, but are not limited to, analysis of volcanic and long period earthquakes (e.g. Chouet et al., 1994; Aki and Ferrazzini, 2000), surface deformation (e.g. Cayol et al., 2000; Lu et al., 2000), anisotropy (Gerst and Savage, 2004; Savage et al., 2015), and of course travel time studies. The two major advantages of using ambient noise, over some of the other methods listed, are the continuity of the dataset and the sensitivity to changes at depth. In this section, mechanisms for velocity change in volcanic systems are discussed (Section 2.2.1), followed by some results from similar studies (Section 2.2.2).

\subsubsection{Physical Mechanism}

Interpretations of seismic velocity changes around volcanoes commonly call upon variations in the distribution of microcracks (Ratdomopurbo and Poupinet, 1995; Gerst and Savage, 2004). Such variations occur due to changes in the stress field at depth as ascending material interacts with surrounding rock (Nur and Simmons, 1969; Ratdomopurbo and Poupinet, 1995; Sparks, 2003). Laboratory experiments have shown that when pressures are increased, seismic velocities increase from the closing of microcracks (Nur, 1971). At larger pressures, new cracks appear as the surrounding rock is deformed (Lockner et al., 1977). Such damage results in a seismic velocity decrease (Nur and Simmons, 1969; Lockner et al., 1977). These responses are expected to be strong in volcanic rocks, which are likely to have a large amount of pre-existing microcracks (Budi-Santoso and Lesage, 2016). To support this, velocity changes have often been observed to coincide with edifice inflation and deflation events (e.g. Patanè et al., 2003; Brenguier et al., 2008b; Duputel et al., 2009; Mordret et al., 2010; Obermann et al., 2013; Bennington et al., 2015). In this scenario, increased fracturing during summit inflation produces a velocity decrease, while subsequent relaxation of the edifice - and closing of cracks - following an eruption results in a velocity increase. Thus, interpretations based on the density of microcracks are often assumed in the absense of a more detailed physical mechanism.

\subsubsection{Similar Studies}

Early observations of velocity changes at volcanoes were based on multiplet analysis and active source experiments at Merapi volcano, Indonesia (Ratdomopurbo and Poupinet, 1995; Wegler and Lühr, 2001; Wegler et al., 2006). These studies detected velocity increases of up to $1.2 \%$ during the 4 months preceding an eruption in 1992 (Ratdomopurbo and Poupinet, 1995) and $0.2 \%$ in the 2 weeks before an eruption in 
1998 (Wegler et al., 2006). Ambient noise based changes were first detected by Brenguier et al. (2008b) at Piton de la Fournaise volcano on Reunion Island. Analysing 18 months of data, they observed velocity decreases of approximately $0.1 \%$ in the weeks preceding four volcanic eruptions. This demonstrated the capability of ambient noise as a potential eruption forecasting tool and, unsuprisingly, led to Piton de la Fournaise volcano becoming something of a central hub for ambient noise based volcano-monitoring (e.g. Duputel et al., 2009; Clarke et al., 2013; Obermann et al., 2013; Rivet et al., 2014; De Plaen et al., 2016). These observations of precursory change set the platform for ambient noise monitoring at volcanoes, paving the way for a global investigation into whether all volcanoes exhibit similar behaviour.

The number of volcanoes that have now been investigated for seismic velocity changes using ambient noise continues to grow. Alongside Piton de la Fournaise and Mt Merapi volcano, examples include Kilauea volcano in Hawaii (Donaldson et al., 2017), Okmok volcano in Alaska (Bennington et al., 2015), Miyakijima volcano in Japan (Anggono et al., 2012) and Mt Ruapehu in New Zealand (Mordret et al., 2010). Successes of these studies have been varied, highlighting the complexity involved when monitoring highly varied volcanic systems. At Mt Ruapehu, for example, a seismic velocity decrease of $0.8 \%$ was detected in the days before a 2006 phreatic eruption, but not for a later phreatic eruption in 2007 (Mordret et al., 2010). The authors interpreted this as possibly related to differences in the time scales of pressurisation, with the 2007 eruption falling outside of the temporal resolution of velocity measurements. In addition, it is becoming increasingly clear that volcanoes are capable of varied deformational patterns (e.g. Anggono et al., 2012; Obermann et al., 2013; Budi-Santoso and Lesage, 2016; Donaldson et al., 2017). Both velocity increases and decreases have been detected before volcanic eruptions, sometimes simultaneously depending on the location sampled (Anggono et al., 2012; Obermann et al., 2013; Budi-Santoso and Lesage, 2016). This can be interpreted to reflect distinct deformational styles depending on the relative position of the source (Budi-Santoso and Lesage, 2016; Donaldson et al., 2017), with regions undergoing contractional and extensional strain simultaneously. Monitoring should therefore look for precusory velocity changes without taking the sign into account. Finally, it is becoming evident that external sources of velocity change - for example through meteorological and tectonic proccesses mentioned earlier - are likely to be present at volcanoes and therefore require separation. Some recent work at volcanoes has therefore looked to remove the effect of non-volcanic background sources in order to better detect changes of volcanic origin (Rivet et al., 2015; Budi-Santoso and Lesage, 2016). Improvements in this area are crucial for realising the potential of ambient noise monitoring at volcanoes. 
The decorrelation of seismic waveforms has recently been used as an additional monitoring tool alongside the measurements of velocity change (Brenguier et al., 2011; Obermann et al., 2013). Temporal changes in the correlation between current cross-correlation functions and the reference function can indicate changes in the scattering of seismic energy (Haney et al., 2015). At volcanoes, this can provide information about structural changes at depth associated with volcanic activity (e.g. Obermann et al., 2013). These changes may be temporary, such as through the opening and closing of cracks, or permanent, through irreversible structural damage. The temporal decorrelation of cross-correlation functions may therefore be a useful tool to support interpretations of behavioural change obtained from velocity changes.

\section{$2.3 \quad$ MSNoise}

Processing raw ambient noise data through to velocity changes typically occurs in multiple stages: (1) waveform preprocessing, (2) cross-correlation computation, (3) temporal stacking and (4) relative travel-time measurement. Such steps are typical, with individual preferences generally related to how these are individually executed. This project uses the Python package MSNoise (Lecocq et al., 2014) to perform the majority of the processing workflow (version 1.4 for Chapter 3 and version 1.5 for Chapter 4). MSNoise provides an integrated solution to go from raw waveforms to measured velocity changes. We use this section to highlight the different processing steps as listed above, including data preparation, and also discuss the different parameter choices available to the user.

\subsubsection{Data Preparation}

Initial input into MSNoise consists of raw seismograms, uniquely identified by the seismic station and recording date. We acquire these seismic data through the GeoNet FDSN web service, downloading day-long raw time series individually for a given station using the Python package Obspy (Beyreuther et al., 2010). Instrument response information for each station is additionally obtained with this download, allowing for subsequent correction after applying a pre-filter between 0.004 and 50 Hz. This is also performed using Obspy. Finally, if the data are split amongst multiple files, these are merged into a single file with gaps padded by zeros. Note that instrument response correction can be performed by MSNoise, in addition to the merging of multiple files recorded on the same day. For simplicity however, these steps are carried out during the download of data. 


\subsubsection{Waveform Preprocessing}

MSNoise performs a number of processing steps prior to computing cross-correlation functions. This aims to remove unwanted signals that may obscure seismic noise, such as earthquakes or instrument irregularities (Bensen et al., 2007). Each 1-day noise trace is first demeaned and tapered. If start times are not compatible with the sample spacing - i.e. not an integer multiple - the data are phase-shifted in the frequency domain. Traces are then bandpassed between a specified frequency range with the option of downsampling available.

If processing station-pairs, north $(N)$ and east $(E)$ components of individual stations are rotated into radial $(R)$ and transverse $(T)$ components prior to performing a cross-correlation. This follows:

$$
\begin{aligned}
& R=N \cos (\phi)+E \sin (\phi) \\
& T=N \sin (\phi)-E \cos (\phi)
\end{aligned}
$$

where $\phi$ is the azimuth between the two stations (Lecocq et al., 2014). For singlestation processing, this step is not required. Day-long traces for each station are
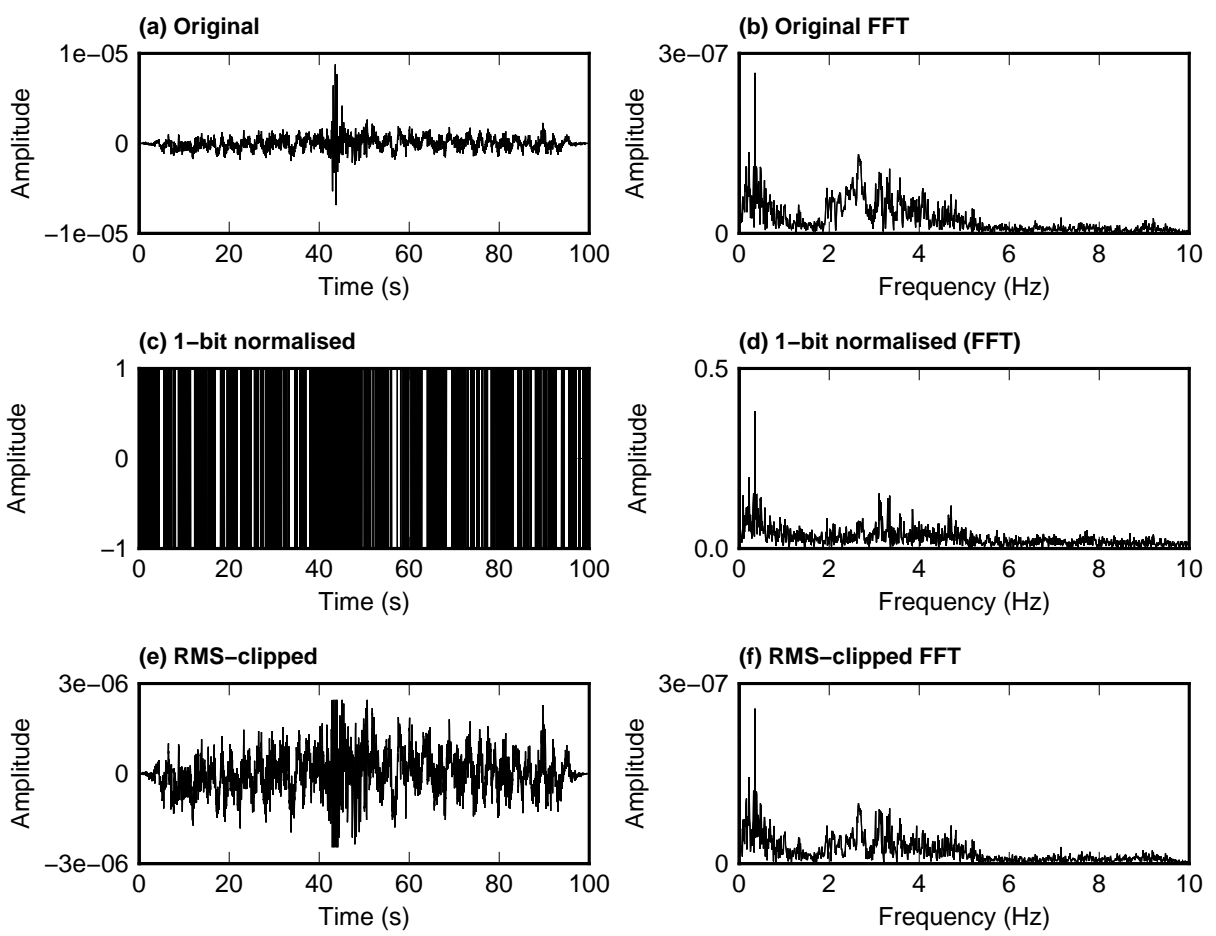

Figure 2.4: Different methods of temporal normalisation are demonstrated, showing the effects in both the amplitude and frequency spectrum: (a) Original raw waveform, (b) FFT of raw signal, (c) 1-bit normalised raw signal, (d) FFT or 1-bit normalised signal, (e) raw signal after being clipped at 3 times the RMS, (f) FFT of RMS-clipped signal. 
then sliced into smaller traces corresponding to a user-defined length, termed the correlation length hereafter. These slices are used to compute the cross-correlation functions. First though, they must undergo temporal and spectral normalisation.

Temporal normalisation is an important step towards surpressing contaminating signals (Bensen et al., 2007). Available options for this stage in MSNoise are shown in Figure 2.4, demonstrated on a trace that contains a clear spike (Fig. 2.4a). One option is to apply one-bit normalisation, where positive amplitudes within the raw trace are replaced with an amplitude of 1 and negative amplitudes an amplitude of -1 (Fig. 2.4c). The new trace then only contains information about the sign of original amplitudes. Alternatively, the user can clip amplitudes to an integer value of the Root-Mean-Square (RMS) of the trace (Fig. 2.4e). We later compare these two options for their effect on the overall stability of cross-correlation functions (Section $3.2 .2)$.

Spectral normalisation, or whitening, acts to broaden the band of ambient noise by equalising the energy across a user-defined frequency band. This combats peaks in the noise amplitude spectrum, as observed in Figure 2.1. Whitening flattens the amplitude spectrum within a desired frequency range while surpressing outside frequencies. The effect of this is shown in Figure 2.5. The main benefit of whitening is to produce cross-correlation functions that are not dominated by energy corresponding to the microseisms (Bensen et al., 2007). Note that we do not perform spectral whitening on auto-correlations, as information is only contained within the amplitude spectrum (Hobiger et al., 2014; De Plaen et al., 2016). Therefore, auto-
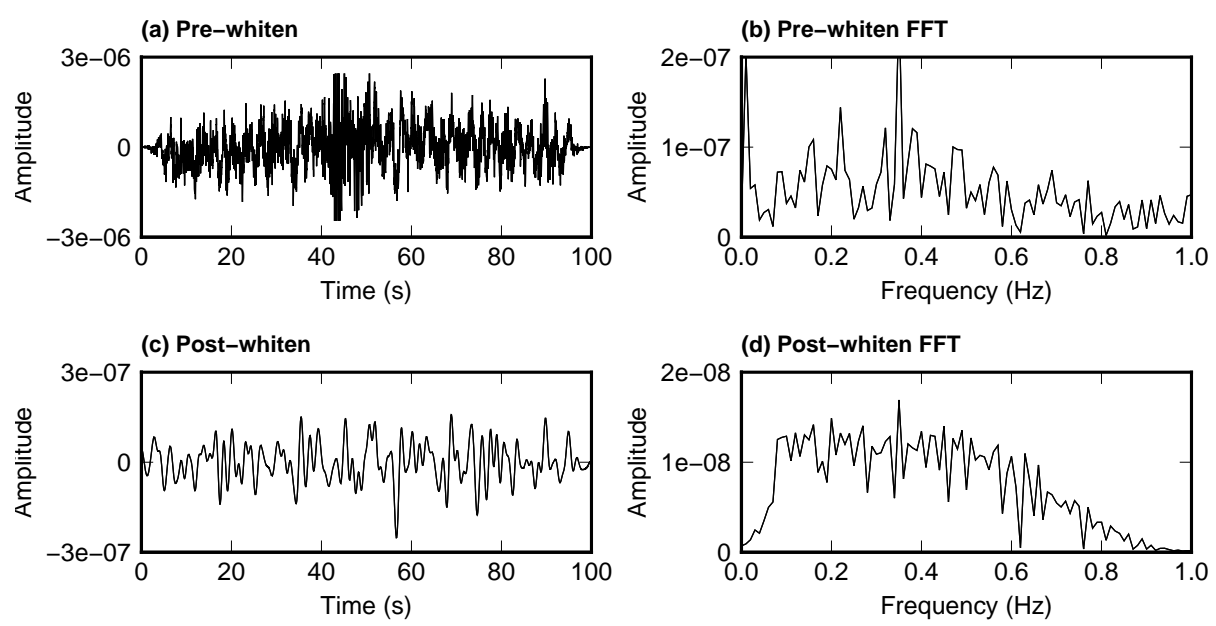

Figure 2.5: Comparison of RMS-clipped signal before and after whitening is applied between 0.1-0.4 Hz: (a) Pre-whitened signal, (b) FFT of pre-whitened signal, (c) Post-whitened signal, (d) FFT of Post-whitened signal. 
correlations are prone to changes in the source of seismic noise (Hobiger et al., 2014).

\subsubsection{Computing Cross-Correlation Functions}

After performing the pre-processing steps in Section 2.3.2, pairs of traces - corresponding to different seismic stations - are cross-correlated. In MSNoise, this is performed in the frequency domain where the correlation operation is defined, for two time series $x(t)$ and $y(t)$ and their corresponding Fourier transforms $X(f)$ and $Y(f)$, as:

$$
C(f)=X^{\star}(f) \times Y(f)
$$

where $X^{\star}(f)$ is the complex conjugate of $X(f)$ (Lecocq et al., 2014). Taking the inverse Fourier transform of $C(f)$ gives the cross-correlation function in the time domain. These are then stacked to produce cross-correlation functions corresponding to a single day. For example, for a correlation length of $N$ seconds, this results in $86400 / N$ traces being stacked for each day. The choice of stacking method is significant. This determines not only how traces are combined into single-day crosscorrelation functions, but also into stacks corresponding to longer time periods, as discussed in the next section (2.3.4).

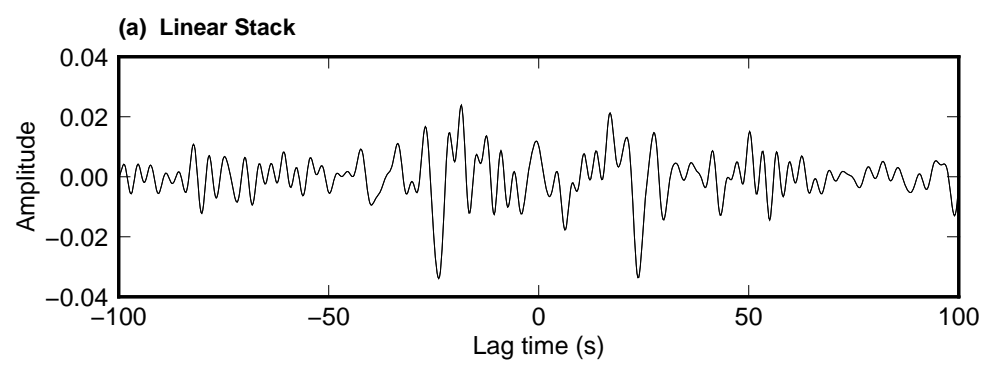

(b) Phase-weighted Stack

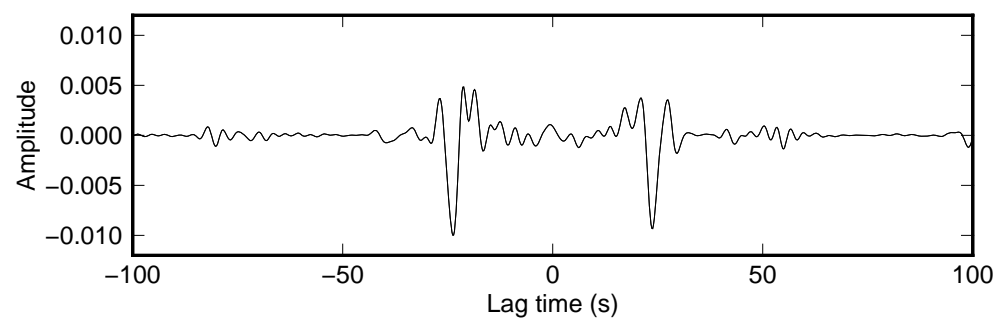

Figure 2.6: Comparison of a linear stack and a phase-weighted stack, with $\kappa=2$, for a one-day cross-correlation between HAZ and WIZ stations. Stacking involves 24 one-hour traces, as defined by a 3600 second correlation length: (a) Linear stack, (b) Phase-weighted stack $(\kappa=2)$ 
Two stacking methods are available in MSNoise, the linear stack and the phaseweighted stack. The linear stack is the more straightforward of the two and simply involves averaging trace amplitudes following:

$$
c(t)=\frac{1}{N} \sum_{i=1}^{N} s_{i}(t)
$$

where $s_{i}(t)$ represents the signals to be averaged and $N$ the number of signals. Alternatively, the phase-weighted stack involves weighting cross-correlation functions based on the coherency of instantaneous phases. The phase stack is defined by (Schimmel and Paulssen, 1997) as:

$$
c(t)=\frac{1}{N} \sum_{i=1}^{N} \exp \left[i \phi_{i}(t)\right]
$$

where $\phi_{i}(t)$ represents the instantaneous phase of the $i=1 \ldots N$ signals. The phase stack in this form originates from the construction of the complex trace $S(t)$ from a seismic trace $s(t)$ following:

$$
S(t)=A(t) \exp [i \phi(t)]
$$

where $A(t)$ is the time-dependent amplitude (Schimmel and Paulssen, 1997). The exclusion of this term in Equation 2.10 means the phase stack being unbiased by amplitude. Finally, the phase-weighted stack produced by multiplying Equation 2.10 by the linear stack:

$$
c(t)=\frac{1}{N} \sum_{i=1}^{N} s_{i}(t)\left|\frac{1}{N} \sum_{j=1}^{N} \exp \left[i \phi_{j}(t)\right]\right|^{\kappa}
$$

where the exponent $\kappa$ allows for the emphasis placed on coherent phases to be customised. Given that amplitudes of the phase stack (Eqn 2.10) vary between 0 and 1 , the result of multiplying the linear stack by the phase stack is to preferentially reduce the amplitudes of the linear stack. Note also that setting $\kappa=0$ simply gives the linear stack. Figure 5 compares both stacking techniques in the computation of a 1-day cross-correlation function from 24 one-hour time slices. Direct arrivals are clearly seen in both cross-correlation functions at about 25 seconds lag — both positive and negative - though are more distinct after performing a phase-weighted stack (Fig. 2.6b). The amplitude of direct arrivals, however, is not a clear identifier of stability for measuring velocity changes, especially when analysis focuses on scattered arrivals. We later test both stacking methods towards improving the temporal 
stability of cross-correlation functions (Section 3.2.2).

\subsubsection{Temporal Stacking}

The main purpose of stacking cross-correlation functions is to improve the signal-tonoise ratio and, therefore, the temporal stability. The expectation is that coherent energy will combine constructively, while incoherent energy combines destructively. An example of 1-day and 30-day stacks are shown in Figure 2.7 over a 6-month period. Note that, in MSNoise, an $N$ day stack for a given day represents the crosscorrelation function for that day stacked with the $N-1$ previous days. Improved temporal stability is clearly achieved by using 30-day stacks (Fig. 2.7b), whereas coherent arrivals are not easily observed at later lag times in 1-day stacks (Fig. 2.7a). However, increased stacking reduces the temporal resolution of the data and therefore the ability to detect short-term velocity changes. The aim then is to choose a stack size that is large enough to recover reliable velocity measurements, but small enough to recover valuable temporal information.

A cross-correlation representing the background state, defined as the reference stack, consists of large number of stacked days such that $N_{\text {Ref }}>N_{\text {Curr }}$; recall, crosscorrelation functions that are individually compared with the reference stack are denoted current stacks. Differences between current stacks and the reference stack

(a) 1 Day Stacks

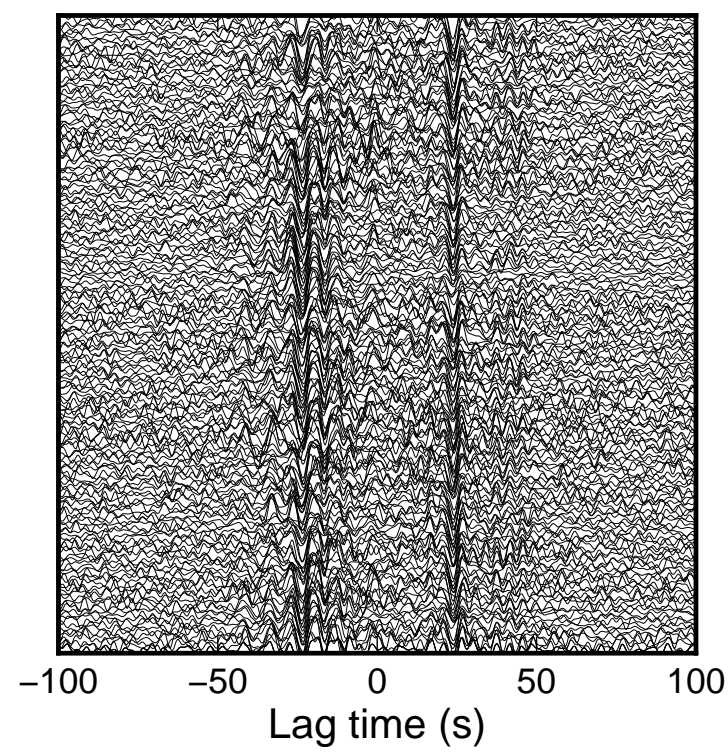

(b) 30 Day Stacks

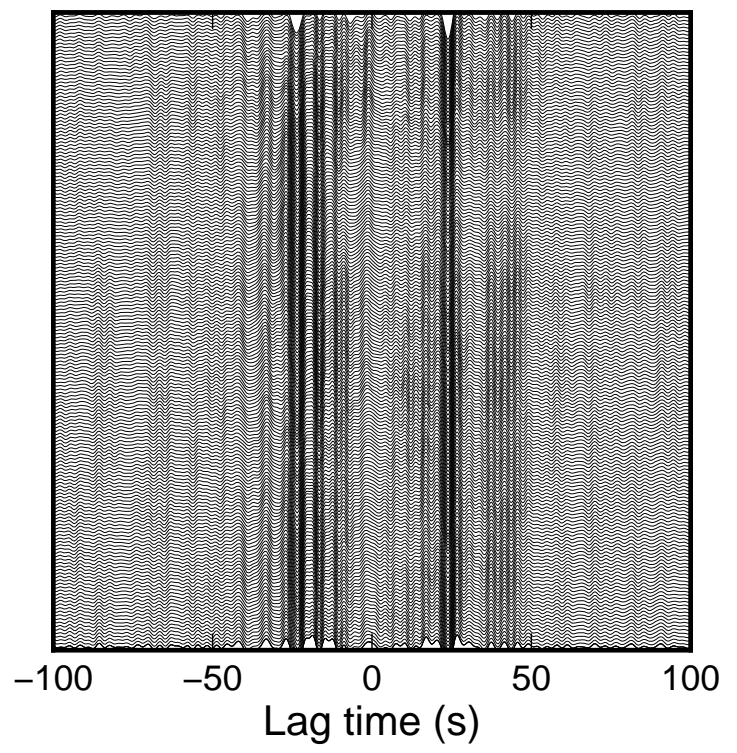

Figure 2.7: Comparison of 6 months of 1-day stacked cross-correlation functions and 30-day stacked cross-correlation functions at HAZ-WIZ station: (a) 1-day stacks, (b) 30-day stacks 
the core of how velocity changes are measured. Therefore the choice of reference stack deserves consideration, with the option to include all available data or a smaller subset of data. We discuss this further in Section 3.2.4.

\subsubsection{Relative Travel-Time Variations}

Velocity changes are determined by computing the delay time between successive current stacks and the reference stack. This is achieved by performing a movingwindow cross-spectrum analysis (Ratdomopurbo \& Poupinet, 1995; Clarke et al., 2011), where delay times are measured in the frequency domain within a user-defined window (Fig. 2.8). The length of this window - in seconds - and the degree of overlap between successive windows are configured within MSNoise. Measured delay times are assigned to the lag time at the center of the window, with repeated measurements producing a distribution of delay time measurements against lag time. The slope of this is then equal to $-\delta v / v$ following Equation 2.5.

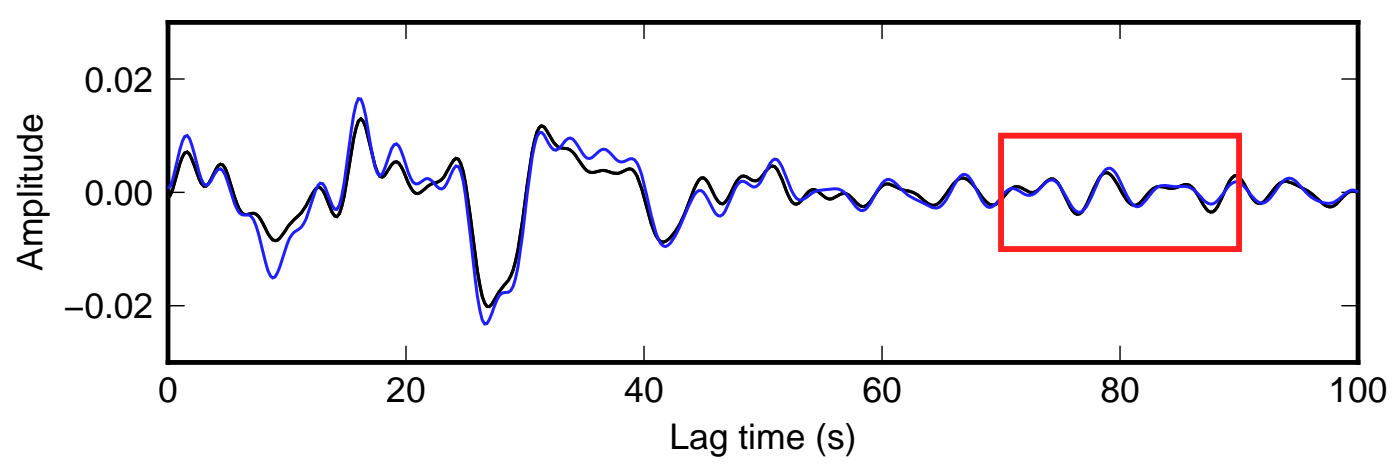

Figure 2.8: An example of a 20-second moving-window (red box) used for measuring delay times between the current 30-day stack (blue) and the reference stack (black).

Delay time measurements are obtained from the cross-spectrum, $X(\nu)$, of the Fouriertransformed current and reference cross-correlation traces (Lecocq et al., 2014). Before transforming to the spectral domain, each windowed trace is demeaned, cosine tapered at both ends by $1 \%$, and padded with zeros (Lecocq et al., 2014). The cross-spectrum is then defined as:

$$
X(v)=F_{R e f}(\nu) \times F_{C u r r}^{\star}(\nu)
$$

where $F_{\text {Ref }}(\nu)$ and $F_{\text {Curr }}(\nu)$ are the first halves of the Hermitian symmetric Fouriertransformed segments of the reference and current cross-correlation functions respectively (Lecocq et al., 2014). The asterisk denotes the complex conjugation. After smoothing the cross-spectrum, through convolution with a Hanning window, the time delay is found in the unwrapped phase of the cross spectrum, $\phi_{j}$, following: 


$$
\phi_{j}=m \nu_{j}
$$

and

$$
m=2 \pi \delta t
$$

where $\nu_{j}$ represents the frequencies sampled in the range $j=l \ldots h$ ( $l$ and $h$ representing low and high frequency respectively) and $\delta t$ is the measured time delay. The time delay between the two signals can be found by performing a weighted linear regression within the frequency band of interest and rearranging for $\delta t$. Weighting follows Clarke et al. (2011), and encompasses both the cross-spectral amplitude and the cross-coherence. This is a measure of similarity between two time series in the spectral domain, defined as:

$$
C(\nu)=\frac{|\overline{X(\nu)}|}{\sqrt{\overline{\left|F_{\text {Ref }}(\nu)^{2}\right|} \cdot \overline{\left|F_{\text {Ref }}(\nu)^{2}\right|}}}
$$

where $C(\nu)$ is the coherence - with values between zero and one - and overlines indicate smoothing (Clarke et al., 2011). The weight $w_{\nu}$, assigned to each sampled frequency $\nu_{j}$ during the regression, is then described by:

$$
w_{j}=\sqrt{\frac{C_{\nu_{j}}^{2}}{1-C_{\nu_{j}}^{2}} \cdot \sqrt{\left|X_{\nu}\right|}}
$$

and the slope $m$ is estimated as:

$$
m=\frac{\sum_{j=l}^{h} w_{j} \nu_{j} \phi_{j}}{\sum_{j=l}^{h} w_{j} \nu_{j}^{2}} .
$$

The slope error is based on the squared misfit of the data $\phi^{2}$ following:

$$
e_{m}=\sqrt{\sum_{j}\left(\frac{w_{j} \nu_{j}}{\sum_{i} w_{i} \nu_{i}^{2}}\right)^{2} \phi^{2}}
$$

and

$$
\phi^{2}=\frac{\sum_{j}\left(\phi_{j}-m \nu_{j}\right)^{2}}{n-1}
$$

where $n$ is the number of different frequencies used in fitting the slope. The final delay time error $\left(e_{\delta t}\right)$ is found by dividing Equation 2.19 by $2 \pi$, following Equation 2.15 .

The final parameter choices involve choosing which delay time measurements are used in calculating the slope of delay times and therefore the change in velocity 
(Equation 2.5). MSNoise allows the user to set a minimum and maximum lag time from which delay time measurements are used. The minimum lag time is usually chosen to avoid direct arrivals, and can be set as a static value or dynamically based on the minimum expected velocity of this direct energy. The maximum lag time, in both cases, is defined by setting a width, in seconds, for the window containing delay times. Additionally, quality control criteria are set in the form of a maximum delay time, maximum error, and the minimum coherence required of measurements used in the linear regression, discussed further in Section 3.2.6.

For this thesis, we modify the base scripts of MSNoise to allow for more flexibility with choices of minimum and maximum lag times, maximum delay time, and maximum error. Specifically, we allow for minimum and maximum lag times to vary for different station-pairs and also different sides of the cross-correlation function (positive and negative lags). The maximum delay time and error are also allowed to vary. The purpose for these modifications is to account for a wide range of station-separation distances, where applying a one-size fits all approach may not be appropriate.

The final weighted linear regression to recover $\delta v / v$ follows (Clarke et al., 2011), taking the form:

$$
\delta t_{i}=a+b t_{i}
$$

where $i$ represents each delay time measurement, and $b$ the slope of delay times $(\delta t / t)$. The coefficient $a$ here allows for possible instrumental drift (Stehly et al., 2007), and can optionally be fixed to zero. The slope $b$ is resolved using a weighted least squares inversion following:

$$
b=\frac{\sum p_{i}\left(t_{i}-\langle t\rangle\right) \delta t_{i}}{\sum p_{i}\left(t_{i}-\langle t\rangle\right)^{2}}
$$

with variance

$$
e_{b}^{2}=\frac{1}{\sum p_{i}\left(t_{i}-\langle t\rangle\right)^{2}}
$$

where weights $\left(p_{i}\right)$ are related to the error of delay times $\left(p_{i}=1 / e_{\delta t_{i}}^{2}\right)$ and $\langle t\rangle$ is the weighted mean of lag times $\left(\langle t\rangle=\sum p_{i} t_{i} / \sum p_{i}\right)$ (Clarke et al., 2011). The intercept $a$ is determined through:

$$
a=\left\langle\delta_{t}\right\rangle-b\langle t\rangle
$$




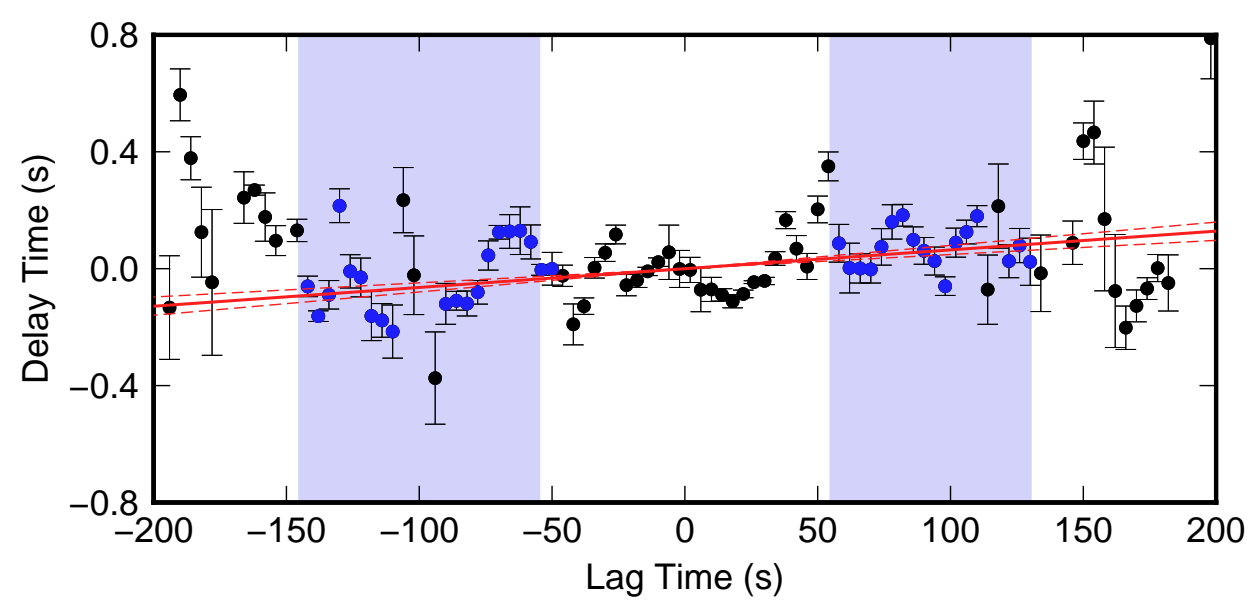

Figure 2.9: Moving-window cross-spectral analysis example, with a slope (red line) and its uncertainty (dashed red lines) fitted to delay times. Delay times used to calculate slope are colored blue while those that fell outside of selection criteria are black. Shaded bars correspond to the chosen minimum and maximum lag times at positive and negative lags.

with variance

$$
e_{a}^{2}=\frac{\left\langle t^{2}\right\rangle}{\sum p_{i}\left(t_{i}-\langle t\rangle\right)^{2}}
$$

where $\langle\delta t\rangle=\sum p_{i} \delta t_{i} \sum p_{i}$ and $\left\langle\delta t^{2}\right\rangle=\sum p_{i} \delta t_{i}^{2} / \sum p_{i}$ (Clarke et al., 2011). Figure 2.9 shows an example of this process, with a slope fitted to delay times within a user defined window. In this particular example, the positive slope indicates a velocity decrease. 


\section{Chapter 3}

\section{Station-Pair Dataset}

This chapter presents the processing steps and results of the station-pair dataset. A significant portion of this is focussed on how processing decisions are justified (Section 3.2). This aims to minimise ambiguity associated with the different ways of processing ambient noise data and the subsequent variability of velocity measurements. This sets a platform for later processing of single-station data in Chapter 4 .

\subsection{Introduction}

The station-pair dataset is made up of ten broadband, three component, seismic stations from the New Zealand Seismic Network (GeoNet). These are used to establish 19 different station-pairs with separation distances ranging from $55 \mathrm{~km}$ to approximately $270 \mathrm{~km}$ (Fig. 3.1). Of these 19 pairs, 16 involve one of the two stations on White Island and therefore represent onshore-offshore noise pathways. The other three station-pairs conversely only involve onshore stations, and therefore represent onshore to onshore paths that pass close to the volcano on a direct path. Our target is to isolate changes in the vicinity of White Island volcano by averaging the results of all of these station-pairs.

Operation times of seismic stations vary throughout the data period (see Appendix A.1 for complete station information). Six have been operating since the beginning of 2007, with WIZ and OPRZ established later in the same year. The remaining stations, HAZ and WSRZ, began operatating in 2010 and 2013 respectively. Note that while WIZ has been recording since 1976, only those data from April 2007 have been downloaded following a sensor change at this time. Thus, the dataset includes 11 station-pairs that run throughout the 2012-2016 eruption sequence with a further eight station-pairs available after the installation of WSRZ station in April 2013. 


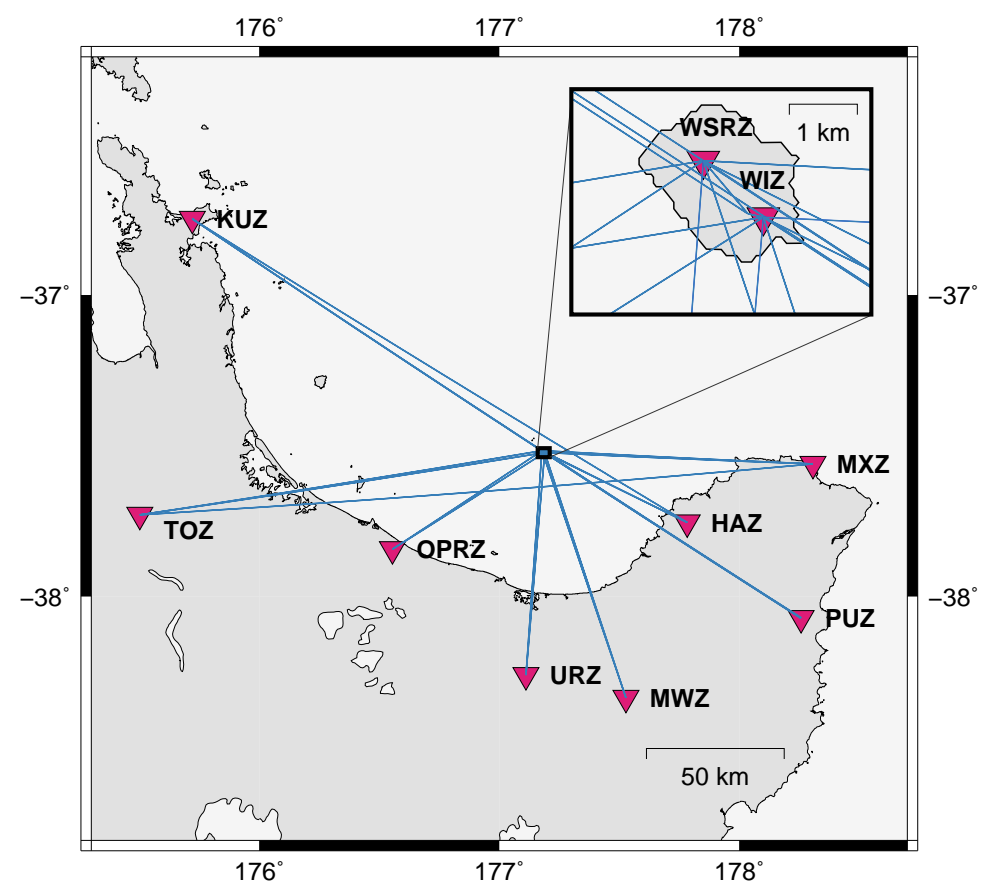

Figure 3.1: Map of study area, showing broadband seismometers (purple triangles) and the corresponding noise pathways (blue lines) between them.

Data are acquired for both horizontal and vertical components at all seismic stations, with the exception of URZ from which only the vertical component data were obtained. We are therefore able to approximate the nine-component Green's function tensor, cross-correlating between all component combinations after conversion to radial and transverse components. The advantage of this is to provide more independent cross-correlation functions, improving the stability of measured velocity changes and the reliability of subsequent interpretations.

\subsection{Parameter Justification}

Numerous processing techniques have been proposed to improve the stability of ambient noise measurements. In practice, it has become evident that any form of optimal processing scheme is dependent on the nature of the dataset of interest (Fichtner et al., 2017). This, in part, originates from the varying degrees to which the data violate the underlying assumptions for Green's function recovery. The primary goal of this section is to investigate and justify available processing options to ensure measured velocity changes are of the highest quality. This aims to minimise the ambiguity often associated with ambient noise work, where processing choices can significantly influence cross-correlation functions recovered (Fichtner et al., 2017).

An iterative approach is applied to investigate parameter choices. This approach involves applying an initial set of parameter choices to a small sample of the data. 
We then look to improve the temporal stability of processed cross-correlation functions by modifying the initial parameters and re-processing the data. The sample dataset consists of one month of vertical component data recorded from 1 June 2010 to 1 July 2010 at all seismic stations excluding WSRZ, which was not active at the time. Data are first bandpassed between 0.01-8.0 Hz, resampled to $20 \mathrm{~Hz}$, 1-bit normalised, and whitened between $0.02-7.9 \mathrm{~Hz}$. Cross-correlations are then computed for 30 minute slices prior to performing a phase-weighted stack (with weighting $\kappa=2)$ to give 1-day cross-correlation functions. This method of stacking is chosen for our initial dataset based on the visual improvement of direct arrivals in Figure 2.6. Following pre-processing, we focus on the decisions involved in processing crosscorrelation functions through to measured velocity changes. For a complete list of the final parameters used, the reader is referred to Appendix A.2.

Parameter justification here is based only on the processing of vertical to vertical component ambient noise data. The adopted processing steps following this section are then used to process the remaining eight component combinations without specific testing. Similarly, it is assumed that parameter choices for station-pairs involving WIZ will have a similar influence to those involving WSRZ. The recovery of coherent energy across all nine component combinations is checked in Section 3.3.1. Note that we were required to apply a time shift correction to computed cross-correlation functions prior to performing the moving-window cross-spectral technique. Details of this correction are found in Appendix A.3.

\subsubsection{Identifying Frequencies of Coherent Noise}

We begin by investigating the frequency dependence of coherent energy in crosscorrelation functions. Specifically, the amplitude of direct arrivals at different frequencies in the cross-correlation function is compared with energy recorded at late lag times. High relative amplitudes of direct arrivals suggest coherent energy is travelling between stations. We use this as a first-order check on whether energy is recorded as intended, noting that large station-separation distances are less capable of recovering short-period energy (Sabra et al., 2005).

Using the methodology of Bensen et al. (2007), a spectral SNR is calculated for the initial dataset to determine the frequencies at which the signal is strongest. This takes a ratio of the peak amplitude measured within a signal window and the root-mean-squared amplitude within a noise window after applying a narrow bandpass filter (Fig. 3.2a). Signal window bounds are defined between $t_{\min }-\tau_{\max }$ and

$t_{\text {max }}-2 \tau_{\max }$ ), where $t_{\min }$ and $t_{\max }$ represent minimum and maximum expected travel times for a given station-pair and $\tau_{\max }$ represents the maximum period of interest, 
set to 40 seconds. Velocity bounds of 1.0 and $3.5 \mathrm{~km} / \mathrm{s}$ are used to determine $t_{\text {min }}$ and $t_{\max }$. Noise window bounds are defined between 360 and 440 seconds lag time for all station-pairs, ensuring no overlap with signal windows. A plot of spectral SNR versus period is then computed by shifting the center period of the bandpass filter in small increments. Note that this classification of the SNR only applies in this subsection, with later measures of the SNR relating to a measure of waveform stability following Clarke et al. (2011). We prepend 'spectral' to the SNR when referencing this specific definition.

(a)

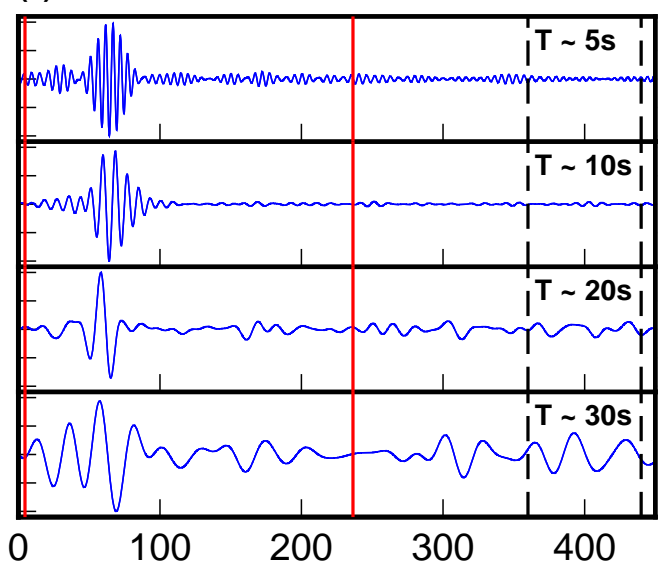

Lag time (s) (b)

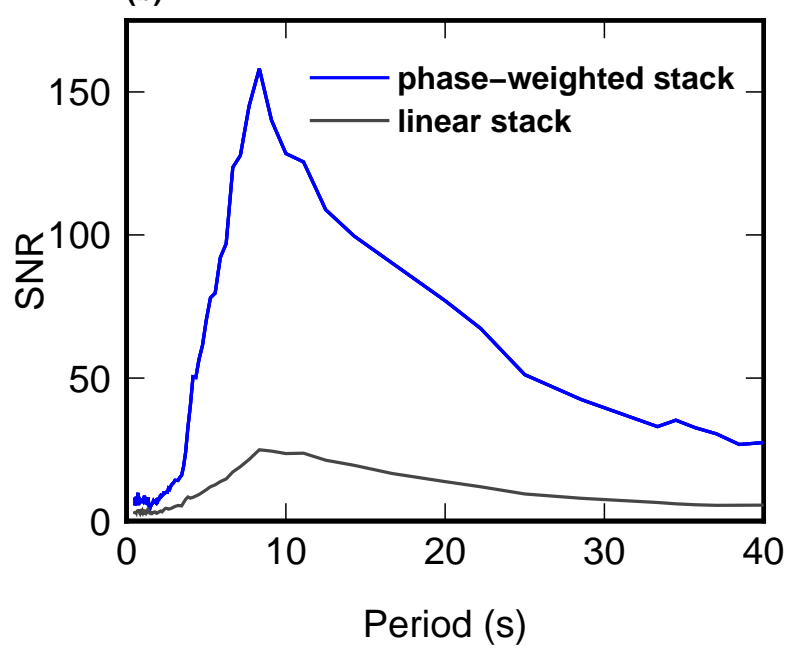

Figure 3.2: Demonstration of how the spectral SNR is calculated: (a) A 30-day phaseweighted stack (positive lag) for station pair KUZ-WIZ is bandpassed around four different center periods $T(5,10,20$ and 30 seconds). Signal window boundaries are defined by vertical red lines and the noise window by dashed black lines. The SNR is then the ratio of peak amplitude within the signal window and the root-mean-squared amplitude of the noise window. (b) The average SNR, across all station-pairs, against period is shown for the 30-day stacked initial dataset. This is computed twice for both a linear (grey line) and phase-weighted (blue line) stack.

The spectral SNR of our initial dataset - averaged over all station-pairs - is shown in Figure 3.2b. This is computed for both linear and phase-weighted stacking of individual days. Phase-weighted stacking produces larger values of spectral SNR compared to linear stacking, indicating higher relative amplitudes of direct arrivals. The highest spectral SNR is observed between periods 5 and 15 seconds (0.06 to $0.2 \mathrm{~Hz}$ ). Below 5 seconds period, the spectral SNR is significantly reduced. This suggests that we can narrow the initial bandpass filter to exclude frequencies where energy is relatively non-coherent. We proceed with an initial $0.01-0.4 \mathrm{~Hz}(2.5-100$ seconds period) bandpass filter and whiten between $0.03-0.35 \mathrm{~Hz}(\approx 3$ to 33 seconds period). Anticipating further iterations, the resampling rate is reduced from $20 \mathrm{~Hz}$ to $5 \mathrm{~Hz}$ for efficiency. 


\subsubsection{Maximising Temporal Stability}

The temporal stability of cross-correlation functions is the most important requirement for measuring reliable delay-times through the moving-window-cross-spectral technique (Hadziioannou et al., 2009; Clarke et al., 2011). Accordingly, a quantatative description of this stability would provide the strongest justification of a given processing sequence. We use the method of Larose et al. (2007) to achieve this, calculating a lag-time dependent SNR based on the variation of individual 1day cross-correlation functions. This was applied directly by Clarke et al. (2011) to investigate the stack size required for realiable delay-times to be measured. Here, we take this further and use the same approach to investigate how the numerous processing choices available influence the stability of cross-correlation functions.

The SNR of a cross-correlation function, produced by stacking $N$ individual days, is given by

$$
S N R(N, t)=\frac{s(N, t)}{\sigma(N, t)}
$$

where $s(N, t)$ is a measure of the signal level and $\sigma(N, t)$ a measure of the noise level. The signal level is determined by taking the Hilbert envelope

$$
s(N, t)=|\langle c c(t)\rangle+i H(\langle c c(t)\rangle)|
$$

where $\langle$.$\rangle denotes the average over N$ single functions $(c c(t))$ and $H($.$) the Hilbert$ transform. The noise level is obtained by measuring the variation at each lag time $t$ between CCFs that make up the stack, following

$$
\sigma(N, t)=\sqrt{\frac{\left\langle c c(t)^{2}\right\rangle-\langle c c(t)\rangle^{2}}{N-1}} .
$$

Both $s(N, t)$ and $\sigma(N, t)$ are smoothed using a 30 second sliding Hanning window prior to calculating the SNR.

We demonstrate the effectiveness of measuring the SNR in this way by using it to justify narrowing the bandpass and whitening bounds. In Figure 3.3a, the average SNR against lag time for the initial dataset is shown after applying the original 0.01-8.0 $\mathrm{Hz}$ bandpass filter. This is lower than the SNR measured after applying the newly defined $0.01-0.4 \mathrm{~Hz}$ bandpass filter dataset (Fig. 3.3b). Thus, we can say the stability of the cross-correlation functions, at least for the sample dataset, has been improved by excluding higher frequency noise. 
(a)

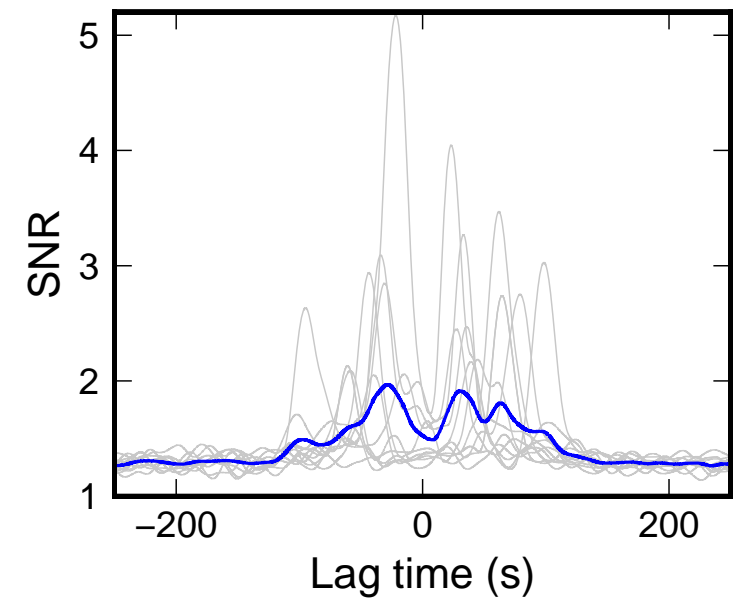

(b)

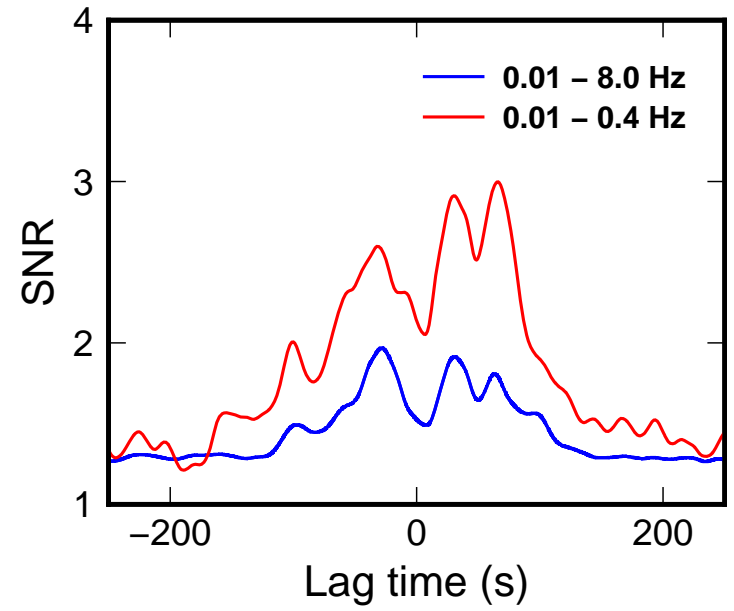

Figure 3.3: The average SNR of 30-day stacks is improved after narrowing preprocessing bandpass and whitening filters: (a) The average SNR of the initial cross-correlation functions, prior to narrowing the filters, is indicated by a blue line. Individual measurements for each station-pair are shown by grey lines. (b) The average SNR across all station-pairs is shown before (blue) and after (red) narrowing the bandpass and whitening filter bounds.

Figure 3.4 demonstrates the iterative testing of key pre-processing parameters, with the aim of maximising the SNR. Testing includes: (1) whether to apply 1-bit normalisation or clip waveforms at 3 times the RMS amplitude (Fig. 3.4a), (2) choosing the length of cross-correlation time slices computed prior to stacking (Fig. 3.4b), and (3) setting the power $v$ of the phase-weighted stack, noting $\kappa=0$ is the linear stack (Fig. 3.4c). Clipping waveforms based on the RMS amplitude, instead of applying 1-bit normalisation, is found to be the most significant factor for improving waveform stability (3.4a). We also find the linear stack results in a higher SNR relative to a phase-weighted stack. This indicates that high amplitude direct arrivals as observed for phase-weighted stacks (Fig. 3.2) — are not a strong indicator of the stability of scatterers. The maximum SNR is achieved by clipping waveforms at 3 times the RMS amplitude, cross-correlating 3600 second time slices and performing a linear stack (Fig. 3.4d).

\subsubsection{Moving-Window Cross-Spectrum Parameters}

In this section, we focus on the parameter choices required for measuring delay times via the moving-window cross-spectrum technique, starting with the choice of reference stack. Two options are considered for this: (1) a reference stack that includes only data recorded prior to volcanic activity, for example, from 1 January 2007 to 1 January 2012 or (2) a reference stack includes all available data. The rationale for 
(a)

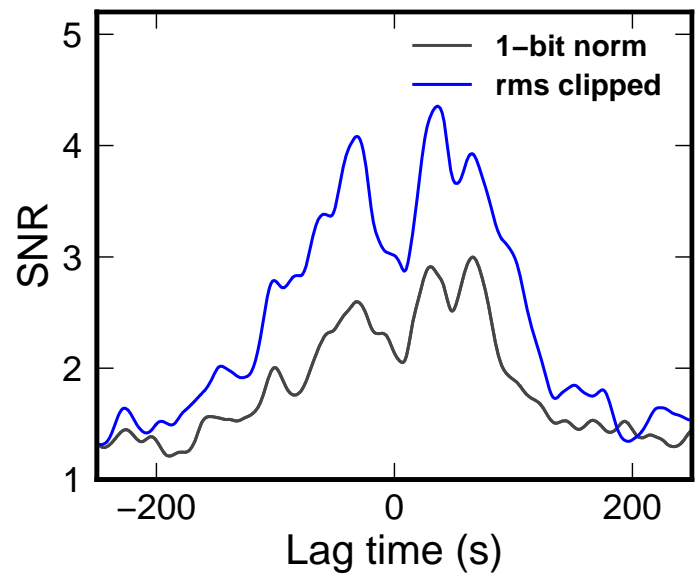

(c)

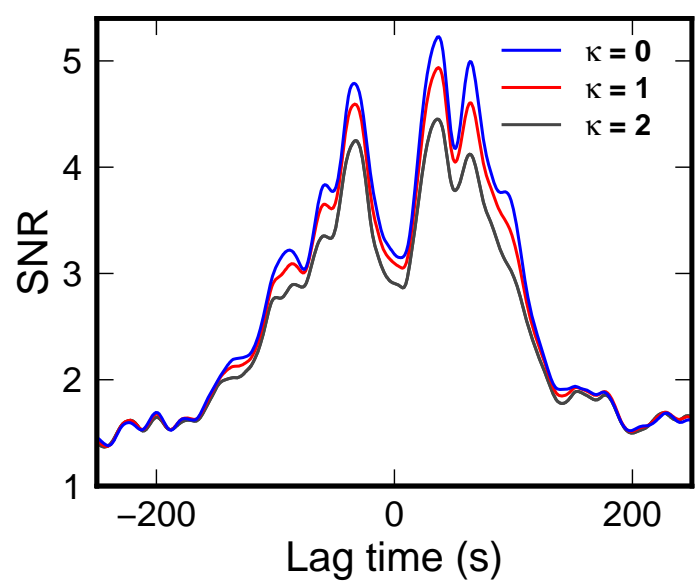

(b)

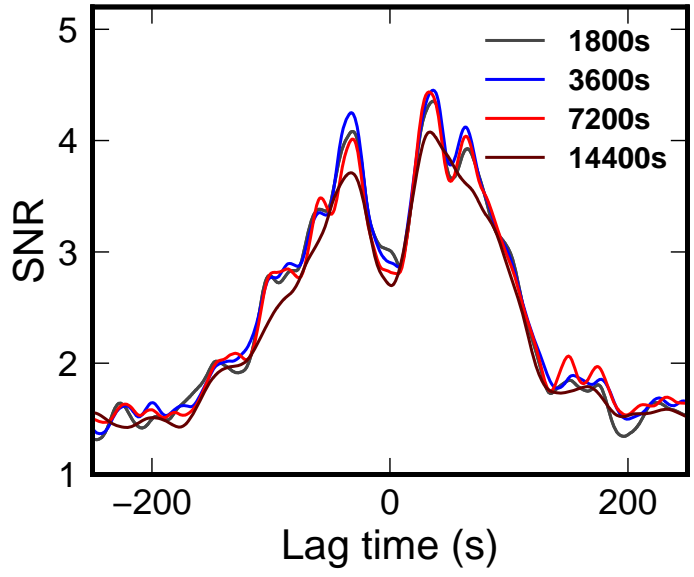

(d)

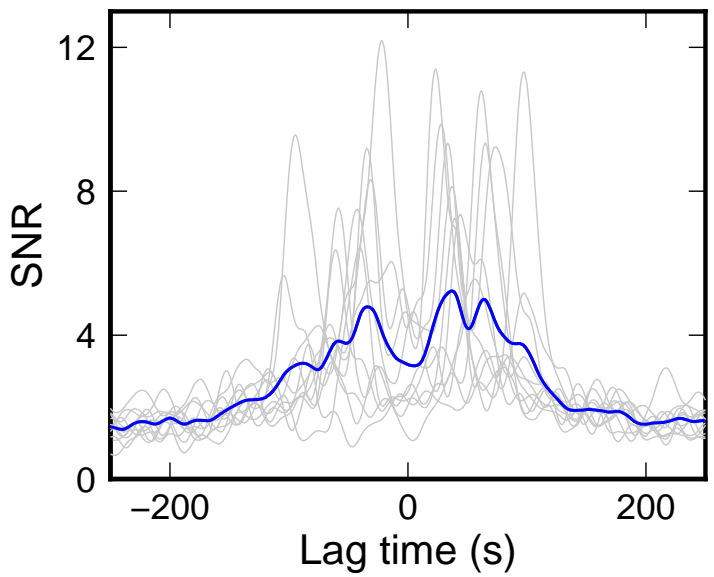

Figure 3.4: Different processing decisions are tested in an effort to maximum the SNR of the initial dataset across all stations: (a) an averaged SNR is shown after applying either 1-bit normalisation or clipping the amplitude at 3 times the root-mean-square amplitude. (b) the length of cross-correlation time slices prior to stacking (to a single day) is varied. RMS clipping of amplitudes is carried through following results in (a). (c) The power $\kappa$ of the phase-weighted stack is varied, with a 3600 second correlation length carried through. (d) The maximised average SNR is shown, after clipping amplitudes to 3 times the RMS, cross-correlating 3600 second time slices and performing a linear stack $(\kappa=0)$. The SNR for individual station-pairs are shown in grey.

using only pre-2012 data is to exclude days in the vicinity of heightened volcanic activity. The reference stack can then be considered to represent a background, low-activity, state. Additionally, the risk of contaminating the reference stack with non-representative cross-correlation functions — produced during active periods is reduced. On the other hand, a reference stack that does not include four years of data, from 2012 onwards, may result in an artificial decrease in waveform coherence in later years. Additionally, better representation during active periods could be more valuable in addressing the objectives of this thesis. 
We use Pearson's Correlation Coefficient (CC) to judge the suitability of the different reference stacks, calculated following:

$$
C C=\frac{\sum_{n}\left(x_{n}-\bar{x}\right)\left(y_{n}-\bar{y}\right)}{\sqrt{\sum_{n}\left(x_{n}-\bar{x}\right)^{2}} \sqrt{\sum_{n}\left(y_{n}-\bar{y}\right)^{2}}}
$$

where $x$ and $y$ represent the two signals to compare, $n$ the number of samples and $\bar{x}$ the mean. The final value falls between -1 , for a perfect negative correlation, and +1 for a perfect positive correlation. Zero then represents no relationship between the two signals. To test reference stack suitability, we calculate the correlation coefficient for 1-day stacks compared with each reference stack. This is done for the entire study period, across all station-pairs, to give a mean-averaged correlation coefficient from 2007-2017 for both a pre-2012 and all-data reference stack.

In Figure 3.5, a residual correlation coefficient is calculated by subtracting correlation coefficients computed using the pre-2012 reference functions from full dataset equivalents. A positive residual then suggests greater similarity - between 1-day and reference stacks — when using all available data. The strongest feature is a permanent increase in the residual correlation coefficient in 2012 (Fig. 3.5), suggesting waveform similarity is artificially reduced from 2012 if using the pre-2012 reference stack. These changes are small however, with the magnitude of the residual within \pm 0.01 . This supports the conclusion that the reference stack has not been significantly altered by including cross-correlation functions computed during active periods. We therefore see no reason to limit the reference function to pre-2012 data, and instead use all available data.

Further parameters to consider include the length of the moving-window, from which delay time measurements are made during the moving-window cross-spectral technique (Section 2.3.5), and the step size between successive windows. A larger window length is expected to provide improved frequency resolution, while a smaller window will provide improved temporal resolution. We test multiple choices of window length on synthetic cross-correlations, with a predetermined velocity change, to address this tradeoff. The choice of window length is then be evaluated based on the recovery of the true velocity. Regarding the step size, it is anticipated that reducing this value will always act to improve the recovery of velocity changes, with more delay time measurements available for the subsequent linear regression. The final choice is then a tradeoff between having more measurements or longer processing times. Going forward, we set the step size between windows to be four seconds.

Synthetic cross-correlation functions are created by stretching the reference func- 


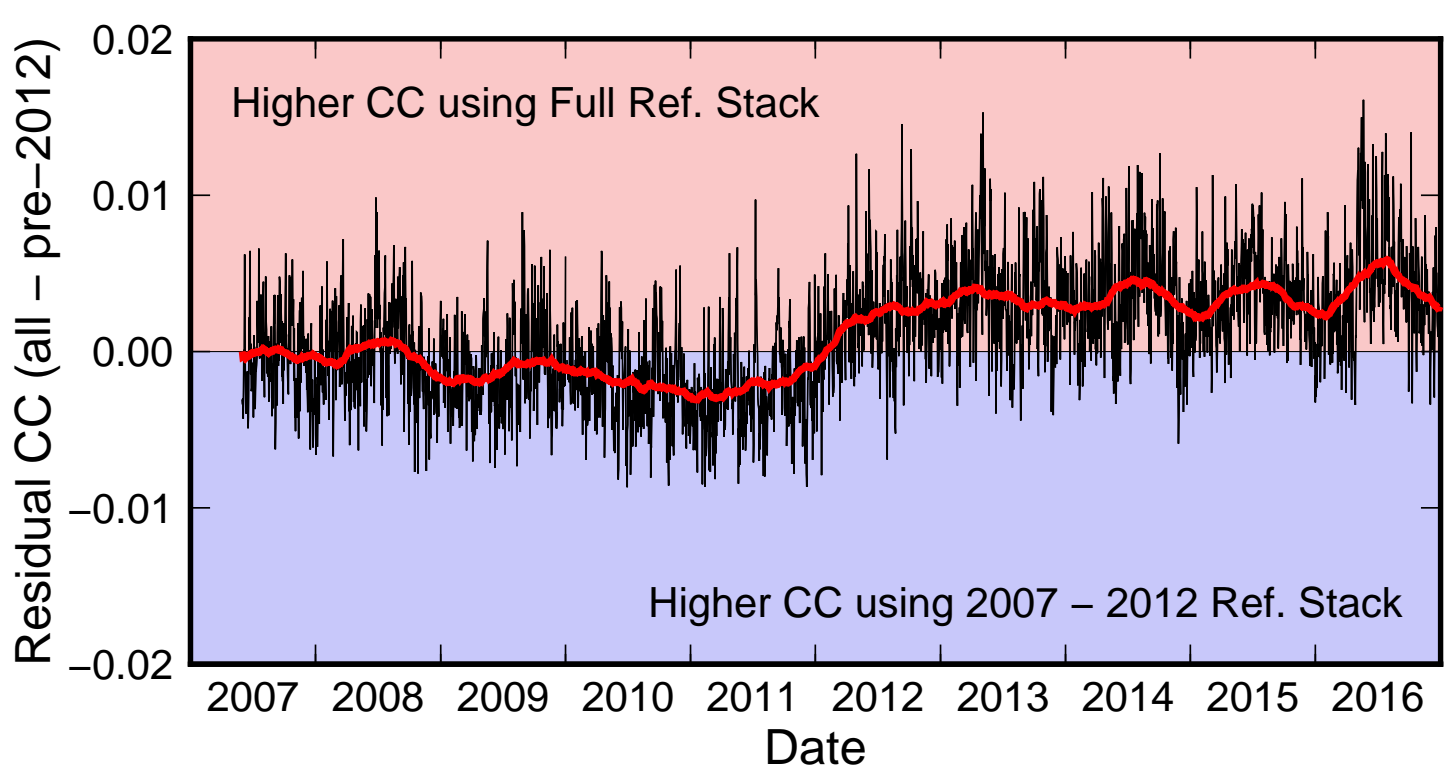

Figure 3.5: Correlation coefficients are measured between individual days and a reference stack across all station-paths (excluding WSRZ) and averaged to give a single CC value per day. This is repeated with two different reference stacks, from 20072012 and from 2007-2017. Here we subtract the 2007-2012 reference stack from the 2007-2017 to obtain a residual correlation coefficient (black line). A 180 day moving window average of this is shown by the red line. When this passes above zero, it suggests waveform similarity, relative to the reference function, is improved with the 2007-2017 reference stack. Below zero suggests improved similarity using the 2007-2012 stack.

tion for station-pair OPRZ-WIZ via a Fourier-transform based interpolation such that they resemble a predefined velocity change. Cross-correlations are resampled following:

$$
N_{\text {syn }}=N_{\text {ref }} \times d v / v
$$

where $N_{\text {syn }}$ is the number of samples in the synthetic function, $N_{\text {ref }}$ the number of samples in the original reference function - before stretching - and $d v / v$ a fractional change in velocity. We induce a velocity decrease of $0.2 \%(d v / v=0.002)$ before introducing noise from real 30-day stacked cross-correlation functions (Fig. 3.6a). Noise is represented by amplitudes between 200 and 500 seconds lag time, which are added to the stretched reference function between -150 and 150 seconds lag time (Fig. 3.6b). It is within these lag times that recovery of the velocity change will be sought. In Figure 3.6c, an example of a synthetic cross-correlation function is shown for a $5 \%$ velocity decrease (for visual clarity). Using this same approach, we create five-hundred synthetic cross-correlation functions. Each of these contains noise taken from a different 30-day stack, such that they are all unique.

The results of testing four different window lengths are shown in Figure 3.7, where 

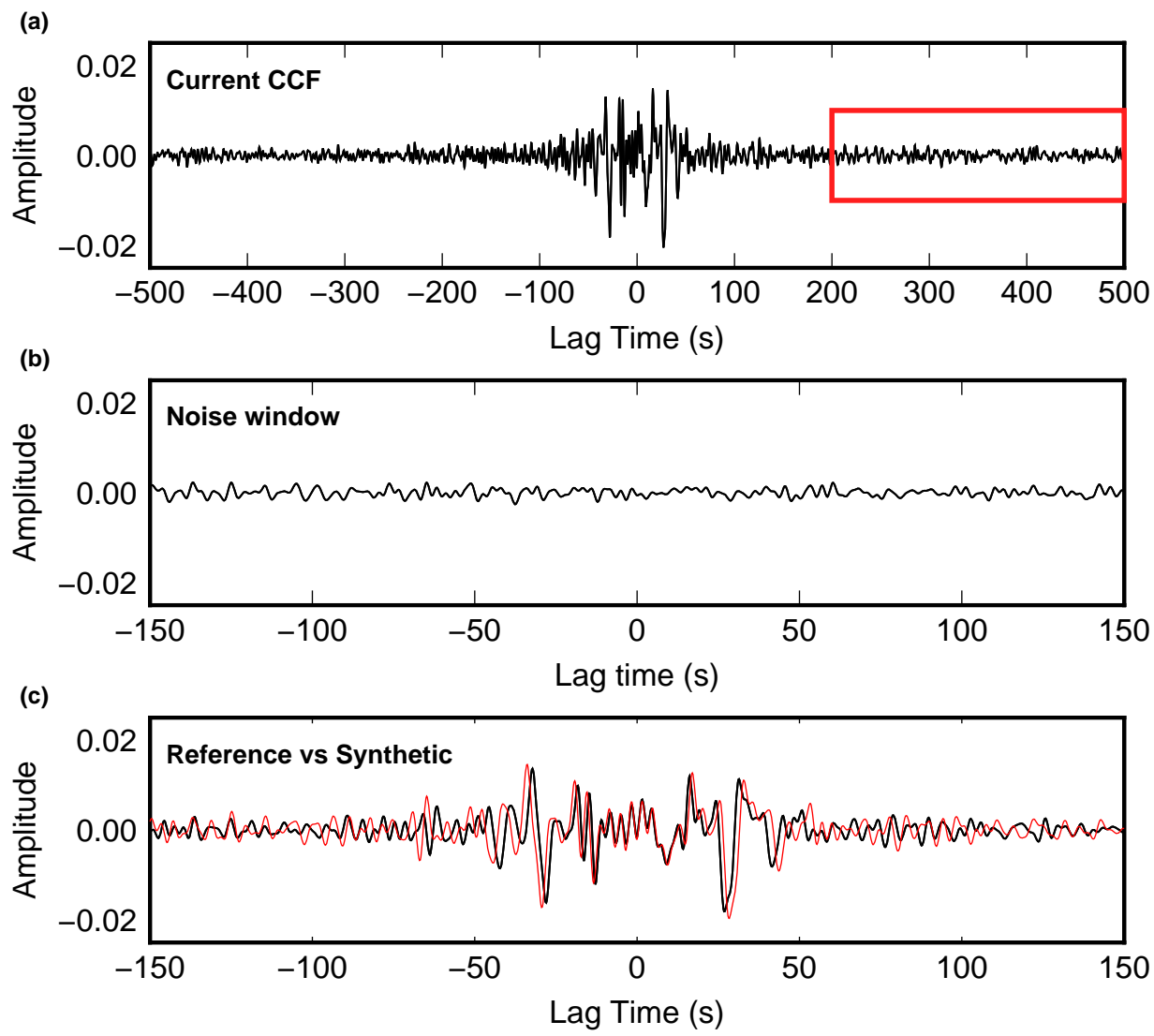

Figure 3.6: Demonstration of how noise is added to a stretched reference stack to produce synthetic cross-correlation functions: (a) Noise is taken from 200-500 seconds lag of a 30-day current cross-correlation function, defined by the red box, (b) A close up of the noise window is shown shifted betwen -150 seconds lag time and 150 seconds. This is added to the stretched reference function. (c) Comparison of a synthetic function (red), representing a velocity decrease of $0.5 \%$, and the original reference function (black).

we attempt to recover a $0.2 \%$ velocity change. Additional parameters used for this purpose only are shown in Table 3.1. We find that as the window length is increased, the recovered velocity approaches the true velocity. For example, a ten second window recovers a mean velocity decrease of approximately $0.16 \%$. A twentyfive second window, conversely recovers the a mean velocity decrease within $0.01 \%$ of the true velocity change. This suggests a larger window is preferable for recovering the true velocity, though it should be considered that a larger window will produce fewer independent measurements. This is important outside of synthetics, where we are limited by a maximum lag time from which reliable velocities can be recovered. We therefore opt for a 20 second window moving forward instead of the maximum tested 25 seconds. 

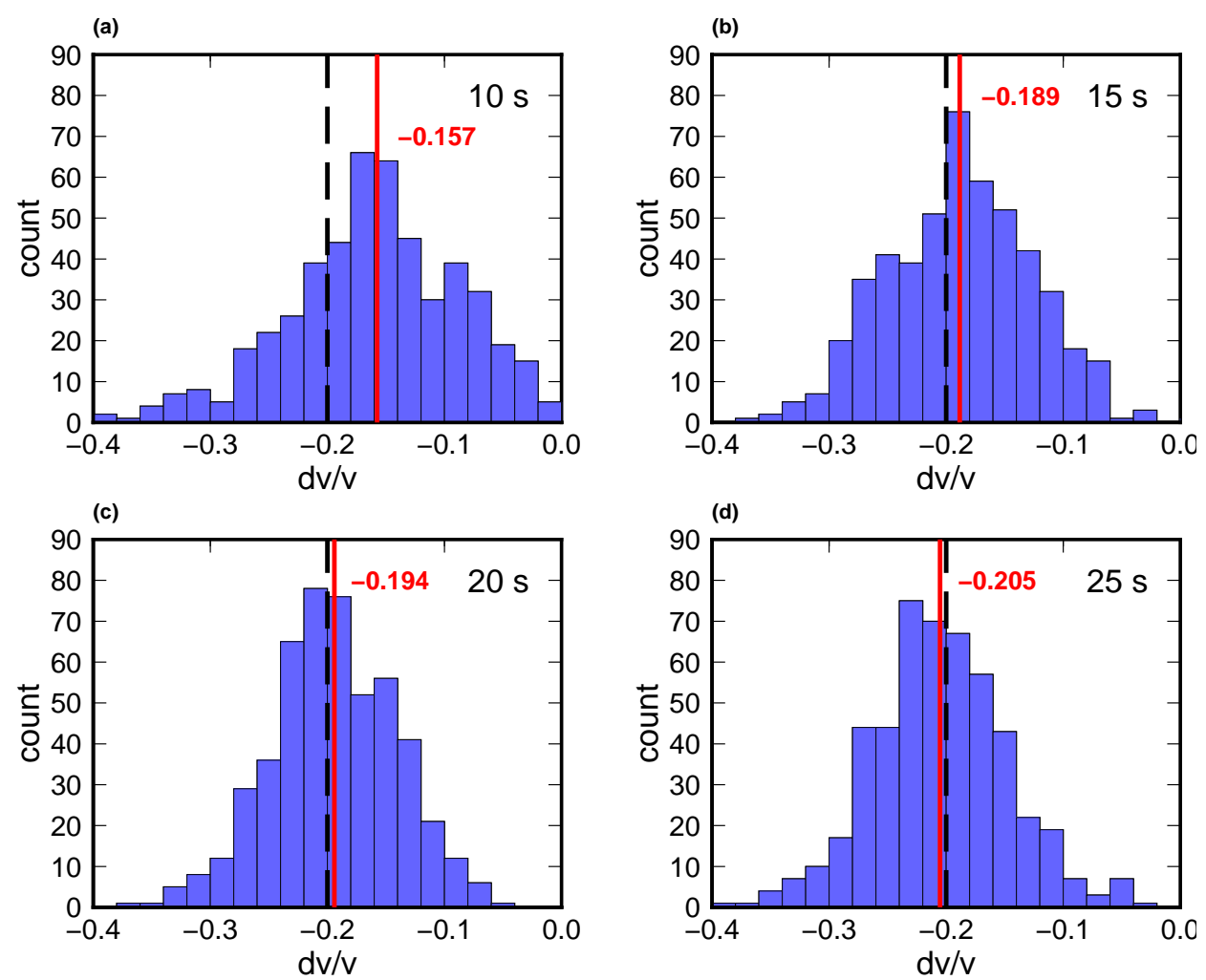

Figure 3.7: Different window lengths for the MWCS stage are tested for their ability to recover a $0.2 \%$ velocity decrease $(\mathrm{dv} / \mathrm{v})$ from 500 synthetic cross-correlations. The dashed-black line shows the true velocity change. The red line shows the mean recovered velocity for the given window length. (a) 10 second window, (b) 15 second window, (c) 20 second window, (d) 25 second window.

Table 3.1: Paramater choices for recoverying velocities from synthetics. These are used only within this section for the purpose of testing window lengths during the moving-window cross-spectral technique.

\begin{tabular}{|c|c|}
\hline Parameter & Value \\
\hline Minimum lag & \pm 35 seconds \\
\hline Maximum lag & \pm 95 seconds \\
\hline Maximum delay & 0.5 seconds \\
\hline Maximum error & 0.1 seconds \\
\hline Minimum coherence & 0.6 \\
\hline
\end{tabular}

\subsubsection{Stacksize Analysis}

In this section, we consider the number of days that should be stacked to accurately recover velocity changes at White Island. Stacking multiple 1-day cross-correlation functions together has the benefit of increasing the SNR. Tomography studies there- 
fore usually combine all available data. However, when looking at time variations, we are required to consider the tradeoff between stacking enough days to accurately measure velocity changes and ensuring suitable temporal resolution. Here, we demonstrate this tradeoff by examining the influence of different stack sizes on the correlation coefficient for one year of data. This allows an informed decision to be made about an appropriate stack size going forward.

The average correlation coefficient for different stack sizes measured from one year of data is shown in Figure 3.8. Using the station-pair OPRZ-WIZ as an example, a significant improvement in waveform similarity is observed when going from 10day to 30-day stacks (Fig. 3.8a). In contrast, the improvement from 30 to 50-day stacks is less significant. To better visualise this, an average correlation coefficient is calculated for the year such that a single value is recorded for each stack size (Fig. 3.8b). Taking the same approach for all other station-pairs, we reveal the relationship between stack-size and SNR for our dataset. As expected, the correlation coefficient increases with stack-size. The most substantial improvements are made
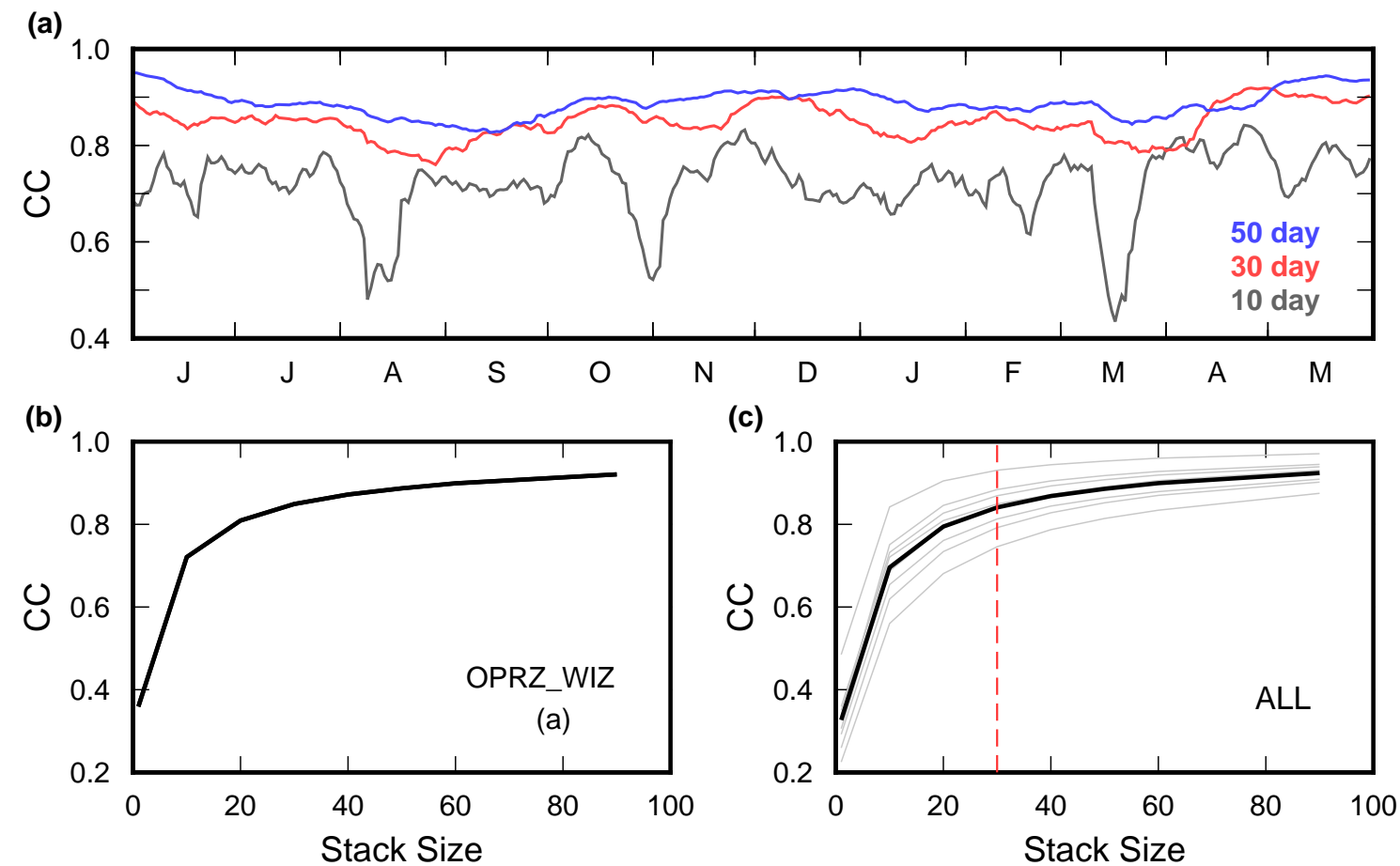

Figure 3.8: Demonstration of the relationship between stack size and the correlation coefficient (CC). The CC is calculated between current and reference stacks at OPRZWIZ using one year of data (1 June 2010 to 1 June 2011). (a) CC is shown for current stack sizes of 10, 30 and 50 days. (b) The average CC for the year at OPRZ-WIZ is calculated and plotted against stack size. (c) The process is repeated for each stationpair (grey lines), with a mean calculated to give a final profile of stack size vs CC. The dashed red line shows the chosen stack size for the station-pair dataset. 
below a stack size of 20-30 days, with further stacking only marginally improving the correlation coefficient. For this reason, a minimum stack size of 30-days is chosen for velocity analysis.

\subsubsection{Relationship between SNR and Coherence}

In this section we consider the relationship between the SNR, computed in preprocessing (Section 3.2.2), and measurements of the coherence output by MSNoise (Eqn. 2.16). Recall, this is a measure of the similarity between the current and reference stack within the moving-window. The coherence is used as a quality control measure, where by a minimum value is defined for accepting delay time measurements. Therefore, we investigate influence of the SNR, as measured earlier, on the coherence. Direct comparison between these two parameters allows for stronger intuition when choosing a threshold value of coherence. We also demonstrate how this information can be used to choose minimum and maximum lag times from which a velocity change is measured.

The SNR and coherence are compared by recording the value of each at the same lag time, allowing for them to be plotted against eachother (Fig. 3.9). In Figure $3.9 \mathrm{c}$, the relationship between these two parameters is observed to be approximately exponential for station-pair MWZ-WIZ. Above a SNR of approximately 2, the coherence begins to fallen out as it approaches a maximum value of one. Conversely, the coherence begins to fall rapidly below a SNR of 2. A similar relatonship is also found using other station-pairs, including those involving WSRZ station (Fig. 3.10).

(a)

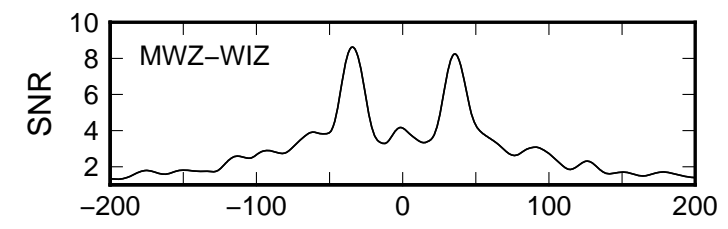

(b)

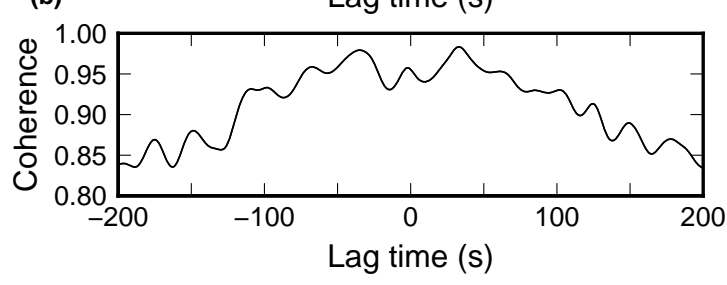

(c)

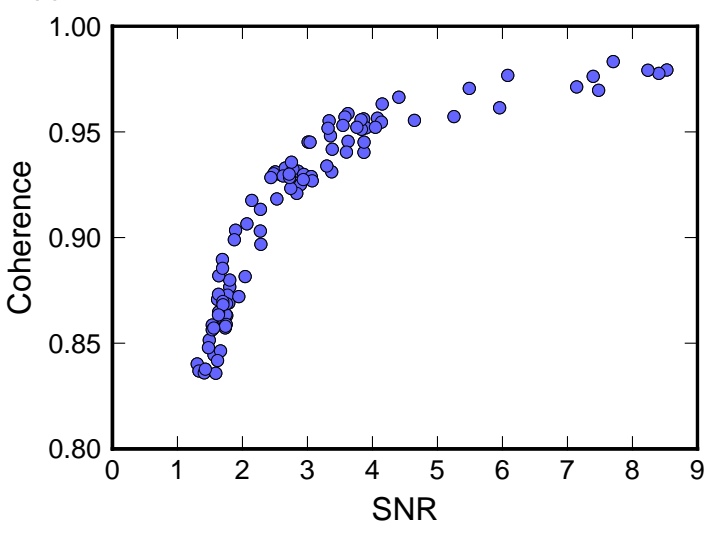

Figure 3.9: An exponential relationship is found between waveform SNR and coherence. (a) MWZ-WIZ averaged SNR for 30 day stacks from 1 June 2010 to 1 June 2011, (b) Average waveform coherence for 30 day stacks at MWZ-WIZ for the same time period, smoothed with a 30 second moving-window, (c) SNR vs Coherence for MWZ-WIZ. 
(a)

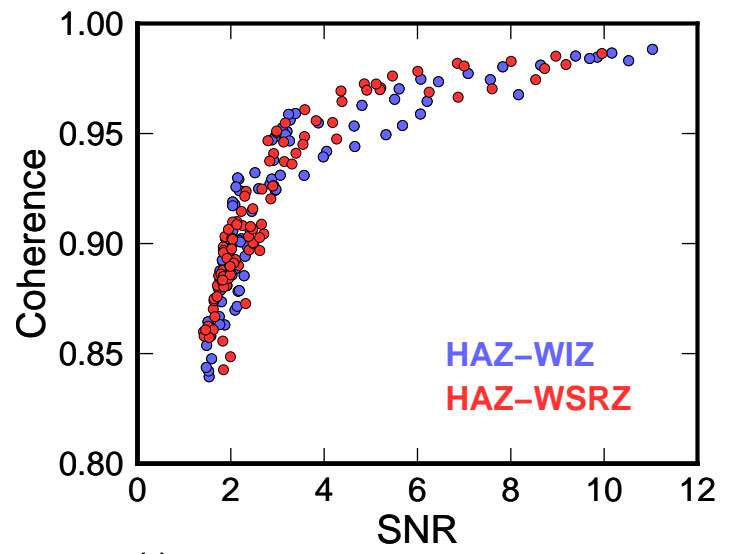

(c)

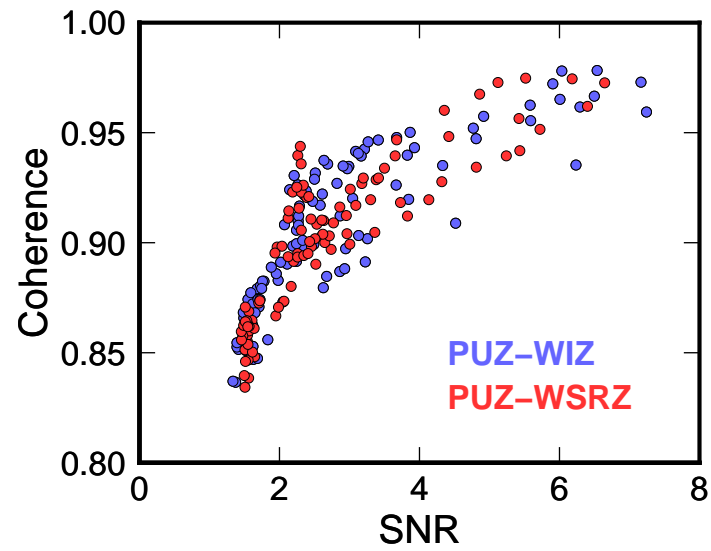

(b)

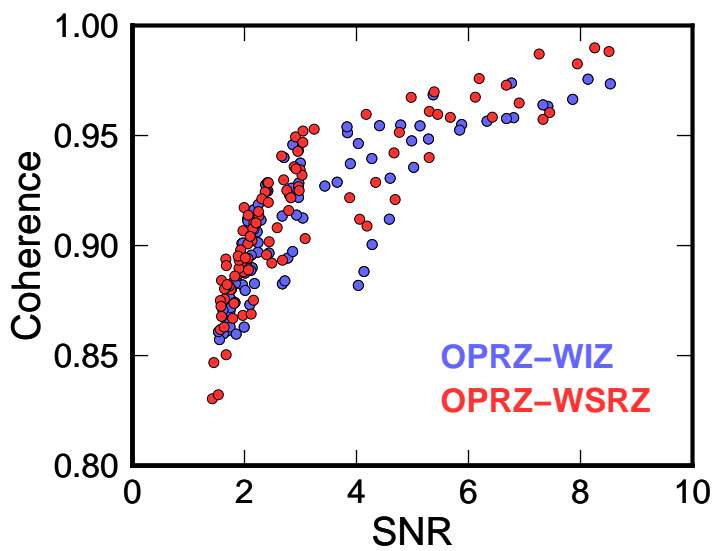

(d)

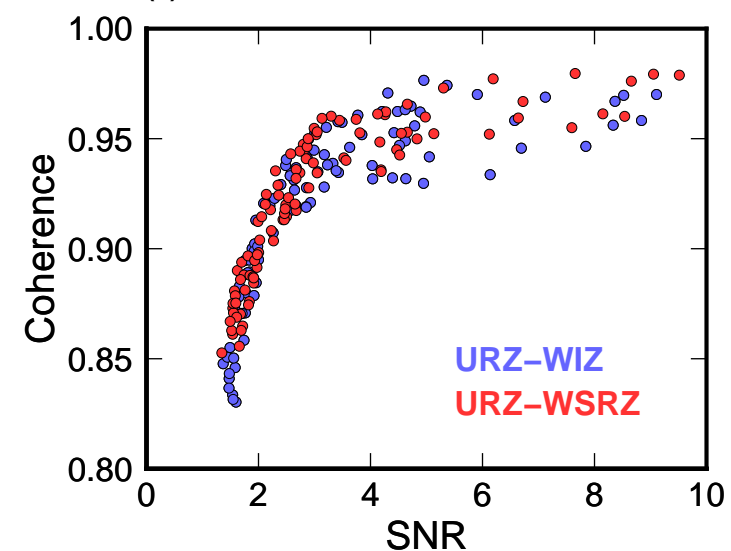

Figure 3.10: Plots of SNR versus waveform coherence for multiple station-pairs. For pairs that use WIZ station, the SNR and coherence of 30-day stacks is averaged over one year from 1 June 2010 to 1 June 2011. For pairs using WSRZ station, the year from 1 June 2013 to 1 June 2014 is used. (a) HAZ-WIZ and HAZ-WSRZ station-pairs (b) OPRZ station-pairs, (c) PUZ station-pairs, (d) URZ station-pairs.

This relationship therefore appears to be stable across the station-pair dataset.

We use a SNR threshold of 2, following the relationship between the SNR and coherence, to determine the minimum and maximum lag times for measuring velocities from delay times. The minimum lag time is defined to avoid direct arrivals. We pick this manually from a phase-weighted stack of all data for each station-pair. This takes advantage of the higher relative amplitudes of direct arrivals when performing a phase-weighted stack (e.g. Fig. 3.2), making them easier to identify. The maximum lag time is defined to exclude data below a SNR of 2. We pick this by looking at the average SNR of 30-day stacks over one year for each station-pair. Figure 3.11 demonstrates minimum and maximum lag times chosen for station-pairs HAZ-WIZ and KUZ-WIZ. The chosen lag times for HAZ-WIZ are shown overlying six months of 30-day stacks in Figure 3.12. For KUZ-WIZ, the minimum and maximum lag times chosen only provide a small window of lag times for velocity analysis (Fig. 3.11c and 3.11d). Rather than comprising the quality of the data, we choose to 
exclude this station-pair. Following this same approach, we reduce the dataset to only include five onshore stations (HAZ, MWZ, OPRZ, PUZ, URZ), with stations KUZ, MXZ and TOZ removed from further analysis. Low values of SNR for these stations is likely a result of long station separation distances. We are therefore left with five unique pairs for each White Island station, giving ten station-pairs overall for the station-pair dataset.

(a)

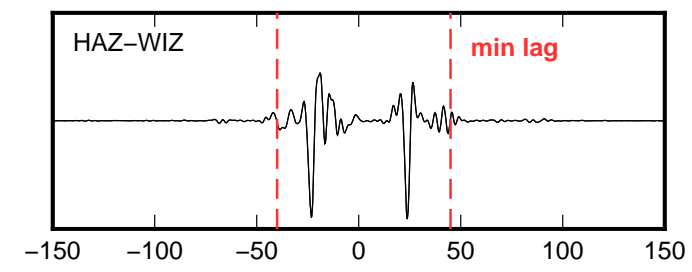

(b)

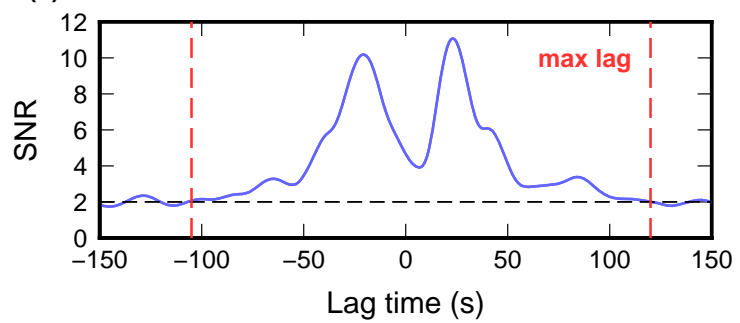

(c)

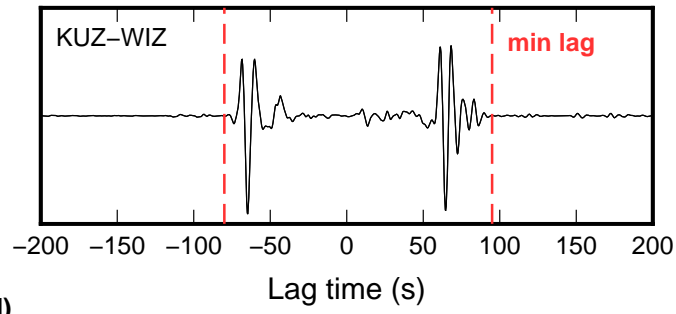

(d)

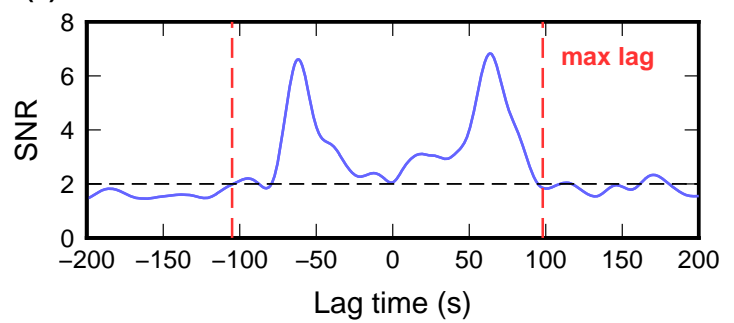

Figure 3.11: Demonstration of how the minimum and maximum lag times are chosen for measuring velocity changes. Minimum lag times are chosen to avoid direct arrivals. Maximum lag times are chosen to exclude data below a SNR of 2 (a) Phase-weighted stack of all HAZ-WIZ data. Chosen minimum lag time is indicated by dashed red lines. (b) Average SNR of 30-day HAZ-WIZ linear stacks from 1 June 2010 to 1 June 2011. Chosen maximum lag time is indicated by dashed red lines. (c) Phase-weighted stack of all KUZ-WIZ data. Chosen minimum lag time is indicated by dashed red lines. (d) Average SNR of 30-day KUZ-WIZ linear stacks from 1 June 2010 to 1 June 2011. Chosen maximum lag time is indicated by dashed red lines. 
(a)
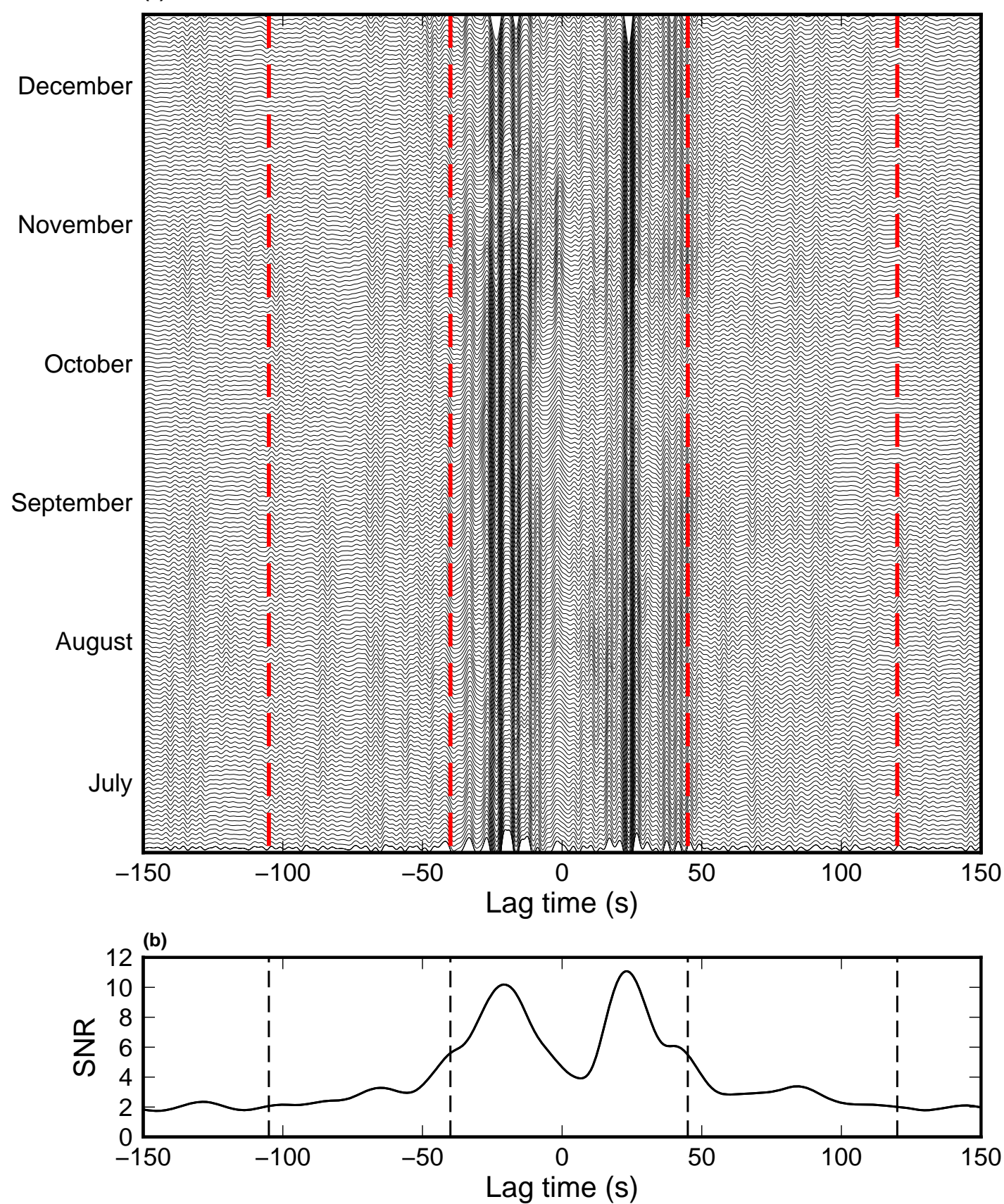

Figure 3.12: Demonstration of chosen windows for station-pair HAZ-WIZ (a) Six months of 30-day stacks are shown from 1 July 2010 to 1 January 2011). Dashed-red lines show the minimum and maximum lag times chosen in Figure 3.11. (b) The SNR from Figure 3.11 is shown with the dashed black lines showing the same windows.

\subsubsection{Final Parameter Choices for Velocity Analysis}

Final parameter choices relate to the screening of poor delay time measurements. These parameters include the maximum acceptable delay time, the maximum error, and the minimum coherence. We use the original approximation of a homogenous velocity perturbation to choose the maximum delay time, recalling that the velocity 
is resolved through the slope of the delay times (Eqn 2.5). From this, the maximum expected delay time for a $0.5 \%$ velocity change is found for each station-pair by calculating:

$$
d t_{\text {max }}=0.005 t
$$

where $t$ is chosen to be the maximum lag time. The choice of $0.5 \%$ as the fractional velocity change is somewhat arbitrary. However, it is considered to be above a maximum that could reasonably be justified as related to White Island volcano given large station distances. with examples in literature typically on the order of 0.1\% (e.g. Brenguier et al., 2008b; Obermann et al., 2013; Bennington et al., 2015). The maximum error of delay time measurements is set to 0.1 seconds. This is small relative to the maximum delay times (Table A.4), ensuring that measurements are unlikely to be within the uncertainty of being either positive or negative. Finally, the minimum coherence threshold is chosen by examining the earlier relationship between the SNR and coherence (e.g. Fig. 3.10). The highest values of SNR are found above a coherence of approximately 0.85. This is therefore chosen as the minimum coherence for the station-pair dataset. A summary of the final parameter choices in this section, specific to each station-pair, is shown in Table A.4.

\subsection{Results}

In this section, we present the results after processing station-pair ambient noise through to velocity changes. First, we describe the process of combining all available data to produce stable measurements of velocity changes (Section 3.3.1). Once satisfied with the final time series, we analyse both short and long-term velocity measurements (Section 3.3.2). We particularly consider the uniqueness of observed changes in the context of understanding background behaviour. Finally, we consider the stability of measurements by looking at dependencies of individual station-pairs on the final averaged velocities (Section 3.3.3).

\subsubsection{Cleaning and Combining Time Series}

The stability of velocity changes is improved by using multiple independent crosscorrelation functions. This section demonstrates how velocity changes recorded by different station-pairs and component-pairs are combined into a single time series for each station on White Island. First though, we show that coherent energy is recorded across all nine component-pairs. This takes into account that parameter justification in Section 3.2 only focussed on the vertical-vertical component (ZZ), with all other component-pairs adopting the same processing steps. 

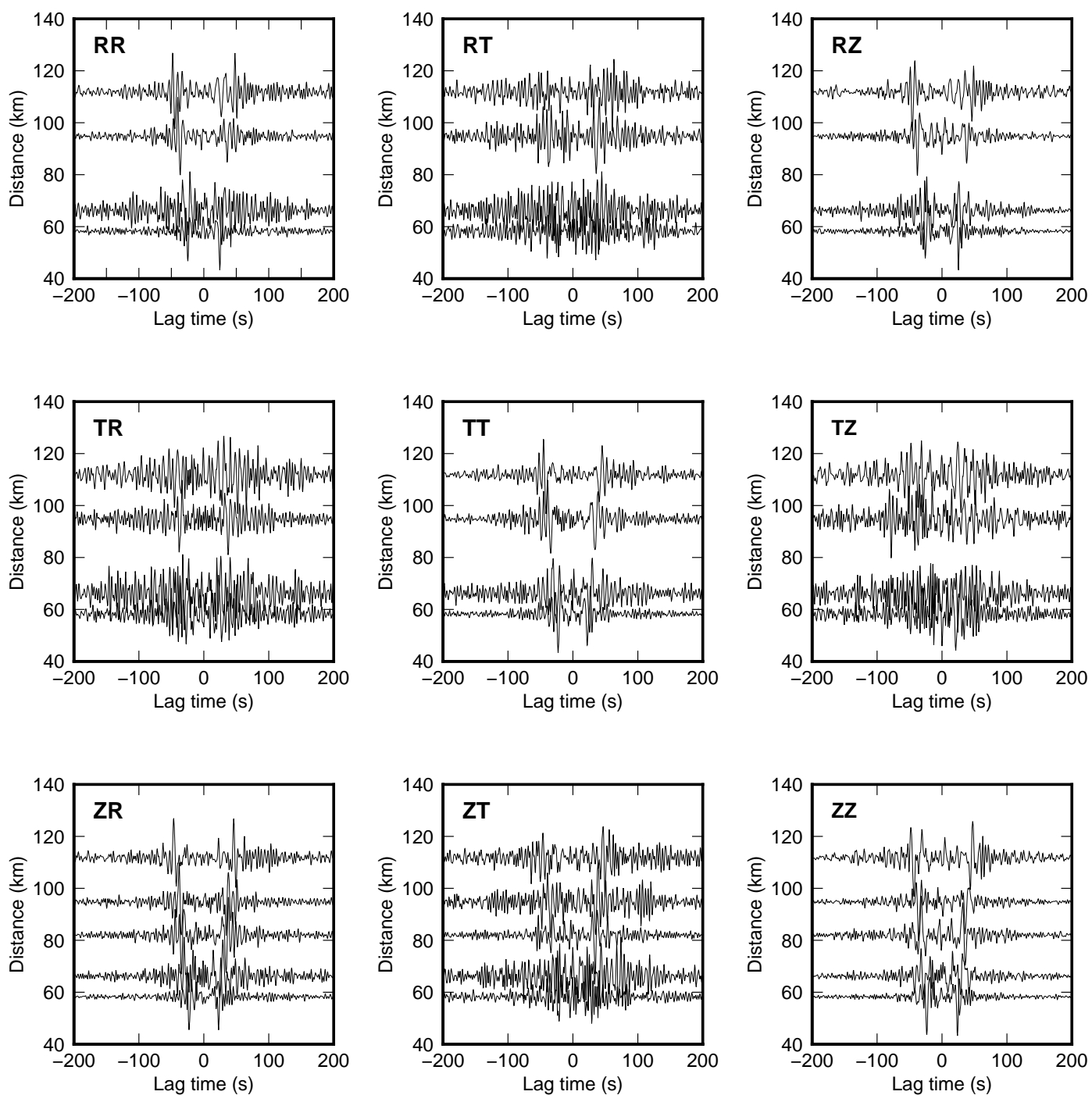

Figure 3.13: Plots of reference cross-correlation functions against station separation distance for all nine component-pairs. Peaks corresponding to direct arrivals are clearly seen for component-pairs that do not include a transverse component.

We look for evidence that coherent energy is recorded travelling between seismic stations across all component-pairs. One approach is to examine cross-correlation functions as a function of station-separation distance. If energy is travelling between stations, we would expect to see direct arrivals at increasingly later lag times as the station-separation distances increase. In Figure 3.13, reference cross-correlation functions are shown for all nine component-pairs, plotted against station-separation distance of the corresponding station-pair. Clear direct arrivals are observed in cross-correlation functions that use the same component at both stations (RR, TT, ZZ). Similar behaviour is also observed in radial-vertical (RZ) and vertical-radial (ZR) cross-correlation functions. However, when a transverse component is crosscorrelated with a vertical or radial component the direct arrivals are no longer clearly observed. This is not surprising, with energy from Rayleigh waves expected to be 
(a)
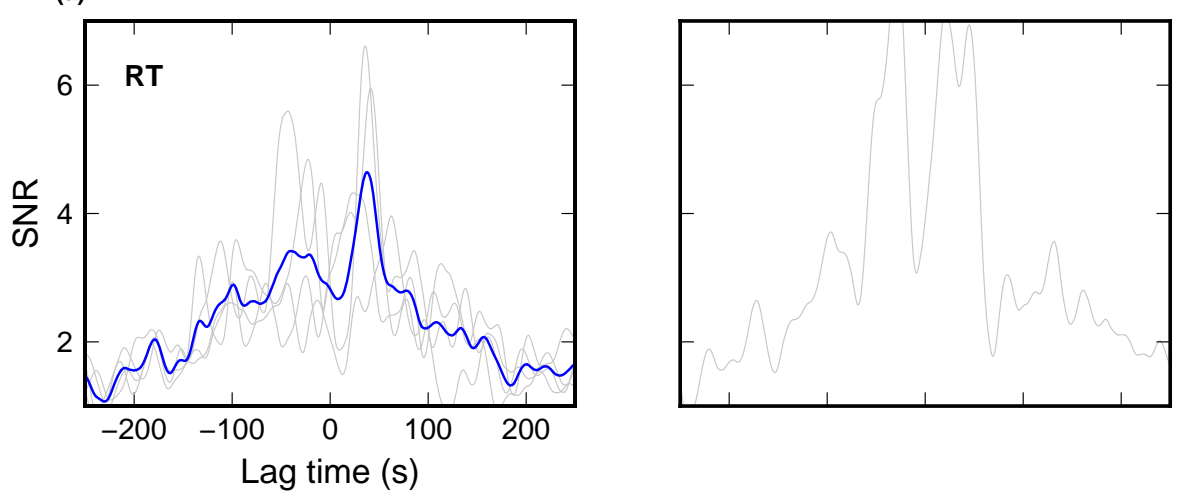

Figure 3.14: Coherent energy is observed for transverse components by plotting the SNR versus lag time of a 30-day cross-correlation funtion. Grey line represents individual station-pairs and the blue line the mean of these. (a) SNR for component-pair RT. (b) SNR for component-pair TR. (c) Average SNR for component-pair TZ, (d) Average SNR for component-pair $\mathrm{ZT}$.

more prominent on vertical and radial components while Love waves are better detected by the transverse component. Thus direct arrivals should not be detected for mixed transverse and radial, or vertical, components. For scattered arrivals however, energy has not followed a direct path between stations. We can therefore expect Rayleigh waves arriving via a non-direct azimuth — not parallel to the radial component - to appear on transverse components and similarly Love waves to appear on radial components. To check components involving a transverse component then, we plot the SNR against lag time of 30-day cross-correlations (Figure 3.14). Peaks are observed at positive and negative lag times that appear to reflect energy travelling between seismic stations. Moreover, energy at later lag times is above the SNR threshold of 2 defined earlier, suggesting that cross-correlation functions are stable enough for measuring accurate delay times. We therefore consider that all component-pairs contain valuable information towards measuring velocity changes.

Combining time series occurs in two steps. First, velocity changes are cleaned to remove unreliable measurements. This involves disregarding measurements made 
within 30 days of either a station coming online or a significant gap (see Appendix A). Second, we calculate a weighted-arithmetic-mean of velocity changes, based on the measurement error of daily velocities computed by MSNoise (Eqn. 2.23). This follows:

$$
v_{d}=\frac{\sum_{i=1}^{n} v_{d_{i}} w_{i}}{\sum_{i=1}^{n} w_{i}}
$$

and

$$
w_{i}=\frac{1}{e_{d_{i}}^{2}}
$$

where $v_{d}$ represents the average velocity change for day $d, v_{d_{i}}$ the velocities for $n=5$ station-pairs and $e_{d_{i}}$ the associated error of these. The variance of the weighted mean is calculated following:

$$
\sigma_{d}^{2}=\frac{\sum_{i=1}^{n} w_{i}\left(v_{d_{i}}-v_{d}\right)^{2}}{\frac{(n-1) \sum_{i=1}^{n} w_{i}}{n}}
$$

with a standard error:

$$
S E=\frac{\sigma_{d}}{\sqrt{n}}
$$

In Figure 3.15b, we represent the standard error by shading a region of $\pm S E$ around the mean line. This represents a $68 \%$ probability that the population mean falls between this zone. An example of an averaged time series for the $\mathrm{ZZ}$ component is shown in Figure 3.15, combining velocity changes from the five station-pairs that use WIZ station. Note that we only use velocity measurements obtained using a fixed intercept of zero during the weighted-linear regression of delay times. Without this condition, days that only had two or three delay time measurements with the remainder not passing quality control criteria (Section 3.2.6) - were artificially recorded as having a small error by MSNoise. When performing a weightedarithmetic-mean, these measurements had a tendency to dominate the resultant time series. Fixing the intercept is therefore preferred here, with only minor differences in the results observed.

Finally, we consider the option to smooth time series in order to better reveal signals of interest. This comes with the expectation that the most short-term velocity changes, on the order of days, are likely representative of noise rather than real velocity changes. In Figure 3.16, two-months of raw velocity measurements are shown from the averaged WIZ time series, overlain by a smoothed representation. Smoothing is achieved by calculating the unweighted mean of values within a centered moving window. Two different moving window lengths are shown in Figure 3.16, 7 days 
(a)

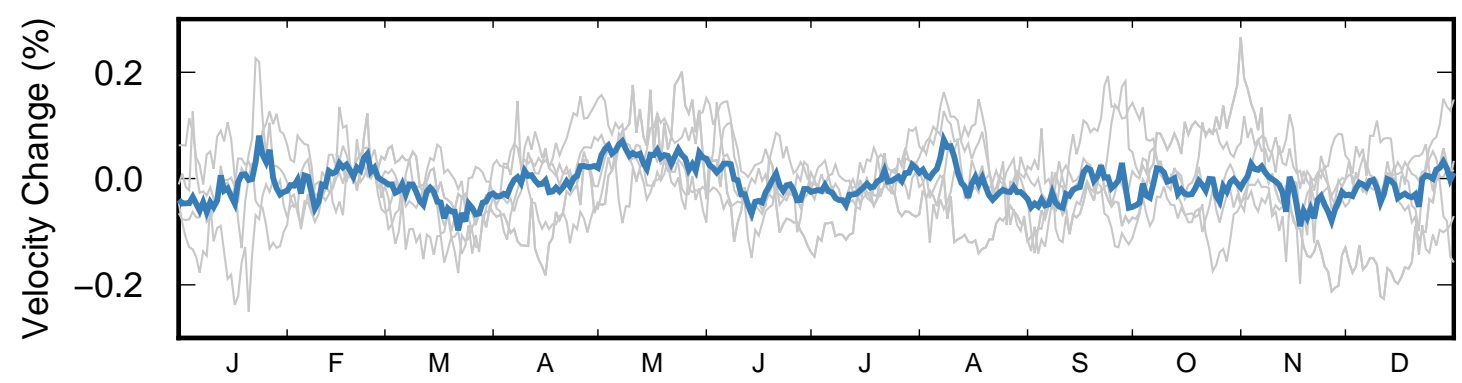

(b)

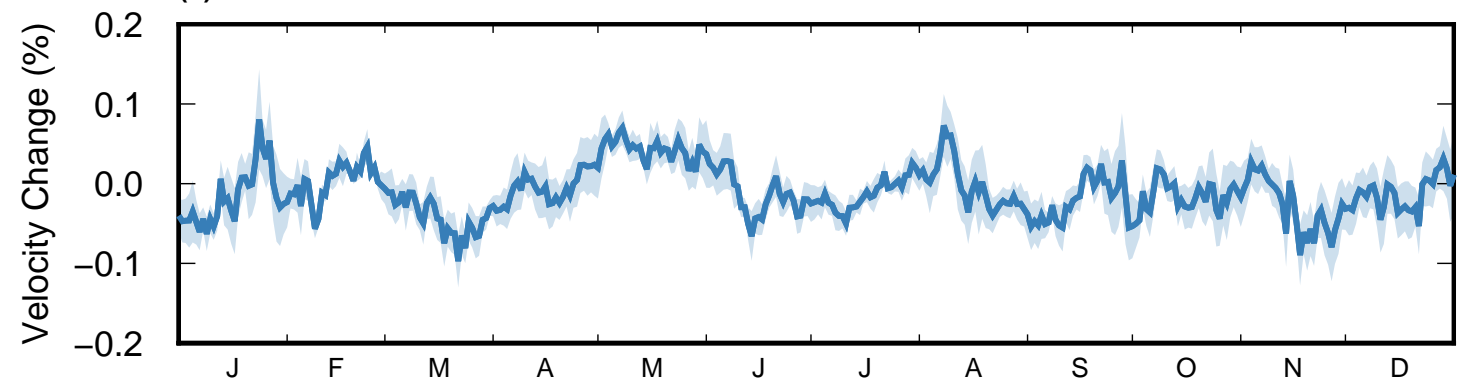

Figure 3.15: Demonstration of how different time series, representing each station-pair that uses WIZ station, are averaged to get a time series representing the verticalvertical component (ZZ). The example shown corresponds to the year 2010 . (a) Individual vertical-vertical component time series for each station-pair are shown by grey lines. The weighted average of these is shown as the blue line. (b) The weighted average is shown again, but with \pm one standard error now shaded around this line (transparent blue).

and 14 days. The 7-day moving window appears to captures the amplitude of peaks well while reducing the amount of scatter. In contrast, the 14-day moving window captures the longer-term trend of the data but does not sufficiently recover the full nature of the peaks. We mostly show velocity changes with a minimum smoothing of 14-days, despite the better apparent fit of a 7-day window. This decision is entirely based on improving the visual presentation of results, with no significant differences observed when comparing the two windows in periods of volcanic activity.

Note that by using a centered moving-window we are not presenting results as could be obtained in real-time. For monitoring, it is preferable to apply a moving window that only encompasses previous measurements. For now though we are interested in representing velocity changes as they occurred, and find a centered window is better suited to this. Application to real-time monitoring is discussed further in Chapter 5.3 .3 . 
(a)

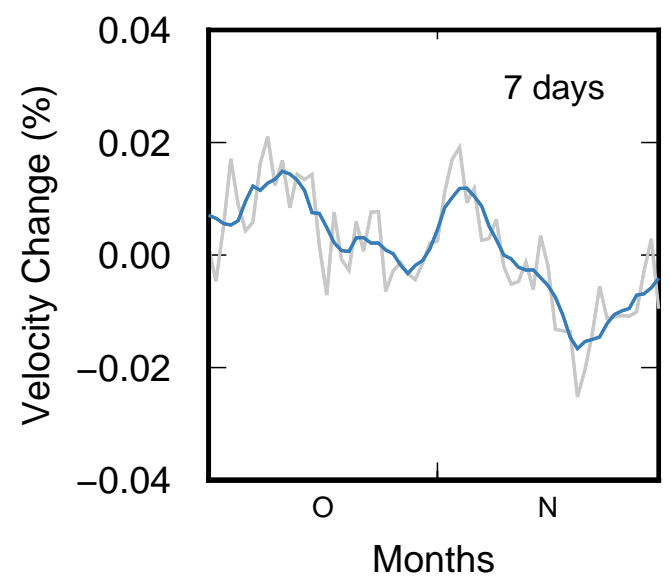

(b)

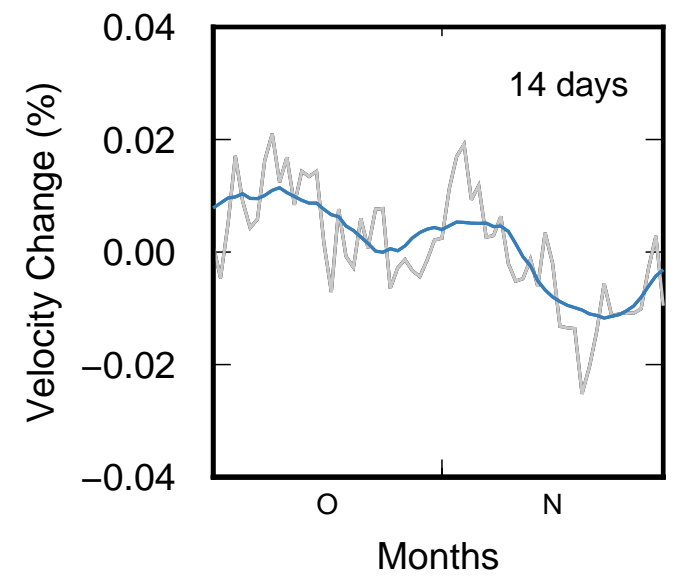

Figure 3.16: Smoothing of time series using a centered moving-window is demonstrated for different window lengths. Two months of data from the averaged WIZ time series is displayed in both (a) and (b). In both cases, the raw, unsmoothed, time series is shown in grey, and the smoothed time series indicated by the blue line (a) 7-day moving-window smooth, (b) 14-day moving-window smooth.

\subsubsection{Velocity Changes}

In this section, velocity changes are presented as obtained through averaging onshoreoffshore noise pathways and the nine different component-pairs (Fig. 3.17). It is expected that through averaging multiple station-pairs that use a common station on the volcano that velocity changes in the vicinity of White Island can be recovered. Thus, within this section, we refer to the average time series for station-pairs involving WIZ station as the velocity changes for WIZ station, and similarly refer to the average time series for all station-pairs involving WSRZ as the velocity changes for WSRZ station (Fig. 3.17). We also calculate the correlation coefficient between current and reference stacks alongside velocity changes, where changes in this parameter can potentially be related to changes in the scattering of energy related to structural changes (Haney et al., 2015). The correlation coefficient is calculated for each station-pair - following Equation 3.4 - between minimum and maximum lag times chosen for delay time analysis (defined in Table A.4). These are similarly averaged for the final time series, though without weighting.

Velocity changes and correlation coefficient measurements for WIZ stations are shown in Figure 3.18a. Of the five events classified as eruptions, as opposed to minor activity or dome growth following Figure 1.3, three occur during a local velocity minimum (Fig. 3.18a). In each case the velocity decreased by approximately $0.02-0.04 \%$ in the weeks preceding the eruption. The magnitude and time scales of these decreases are not unique however, with many instances of similar, or greater, 
(a) WIZ time series

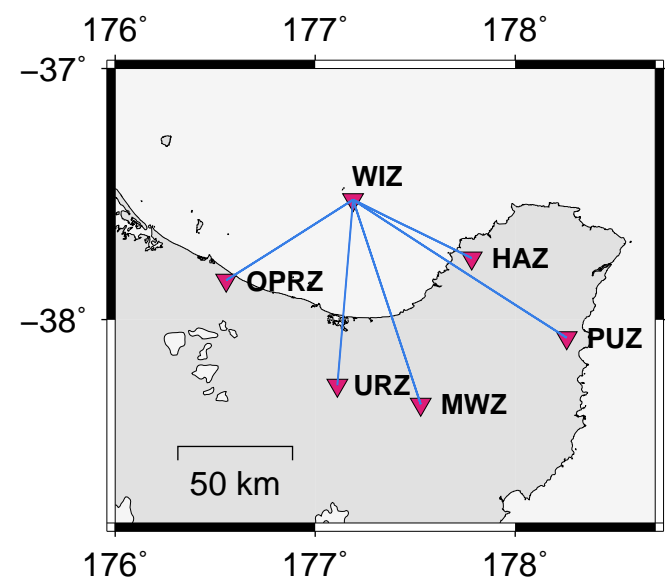

(b) WSRZ time series

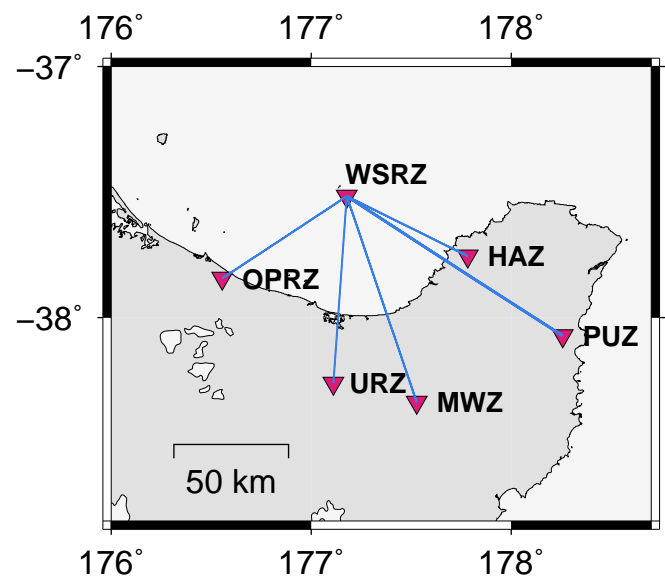

Figure 3.17: Map of station-pairs used to produce an averaged time series for: (a) WIZ station and (b) WSRZ station.

changes throughout the dataset. For example, some of the largest velocity changes occur in 2007-2008, when no eruptive activity was reported. The largest velocity change occurs at the start of 2010 , with fluctuations of approximately $0.06 \%$. This is associated with a significant decrease in the cross-correlation coefficient however, and may therefore represent a period of reduced SNR (Fig. 3.18c). Other velocity changes around eruptive periods include velocity increases preceding the August 2012 and 2013 eruptions. For the August 2012 eruption, the velocity increased by approximately $0.04 \%$ over the $2-3$ months before the eruption. While a change of this magnitude is not unique, the wavelength of this feature is larger than any other change of this magnitude, from the velocity low 3 months earlier to the velocity low 2-3 months later. Velocities during this time are at their lowest since 2008, coinciding with the period of suspected dome growth. Despite this, none of these short-term changes are obvious in their relation to volcanic activity, due similar amplitudes in background fluctuations throughout. Two large velocity changes are observed however that appear semi-permanent, with velocities remaining stable following the change (Figure 3.18a). The first occurs in September-October 2008 with a rapid velocity increase of $0.04 \%$. This is preceded by an apparent long-term velocity decrease through 2007-2008 and remains elevated following the increase through to at least 2012. The second major change occurs at the time of the September 2016 eruption, with a velocity decrease of approximately $0.05 \%$ that shows no recovery by the end of 2016 (Fig. 3.18a). We also see a decline in the correlation coefficient starting around the same time, again with no recovery by the end of the year (Fig. $3.18 \mathrm{c})$. The combination of a semi-permanent velocity decrease and a decrease in the correlation coeffient make this period unlike any other observed between 2007-2017 at WIZ. 
(a)

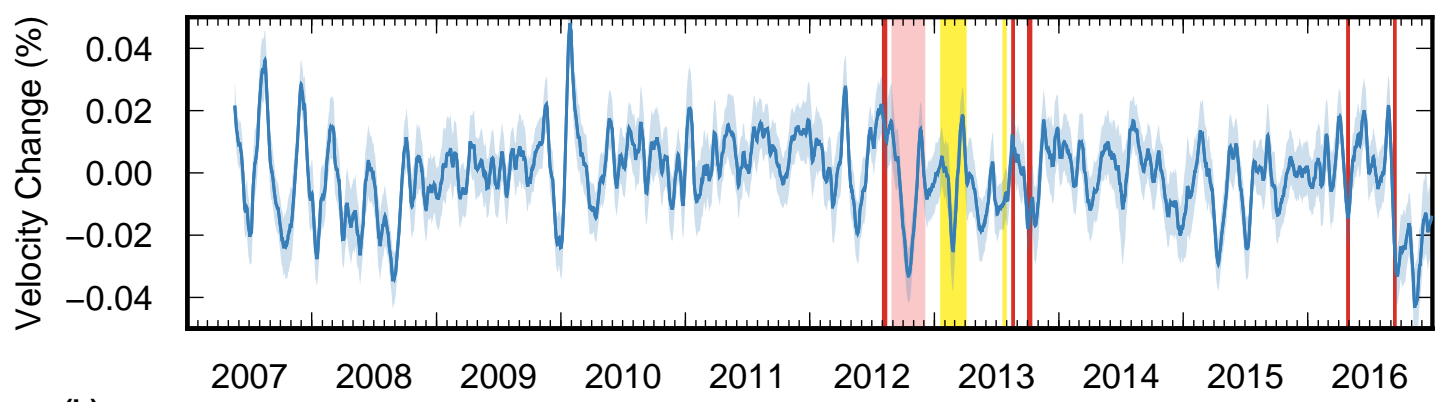

(b)

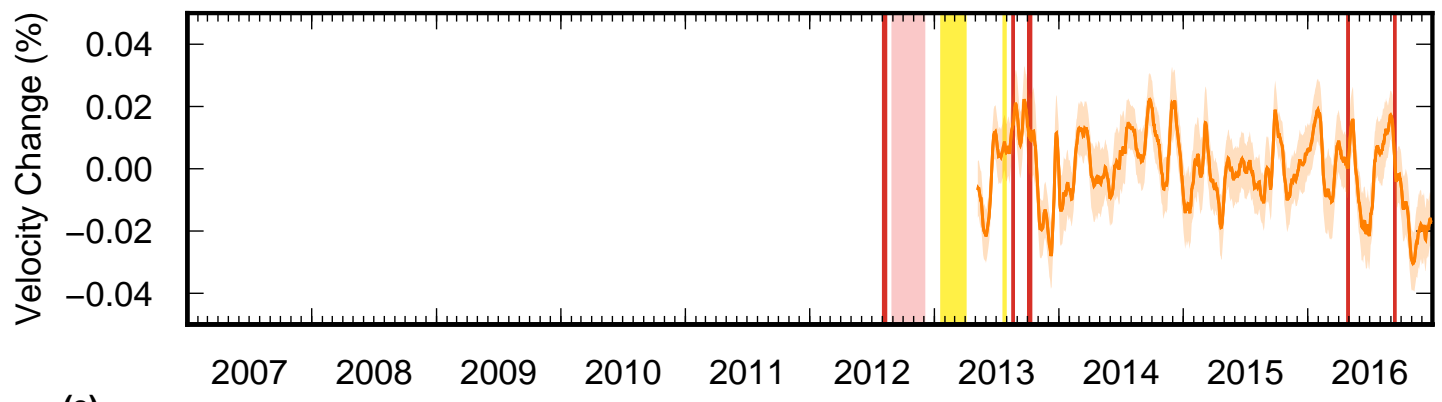

(c)

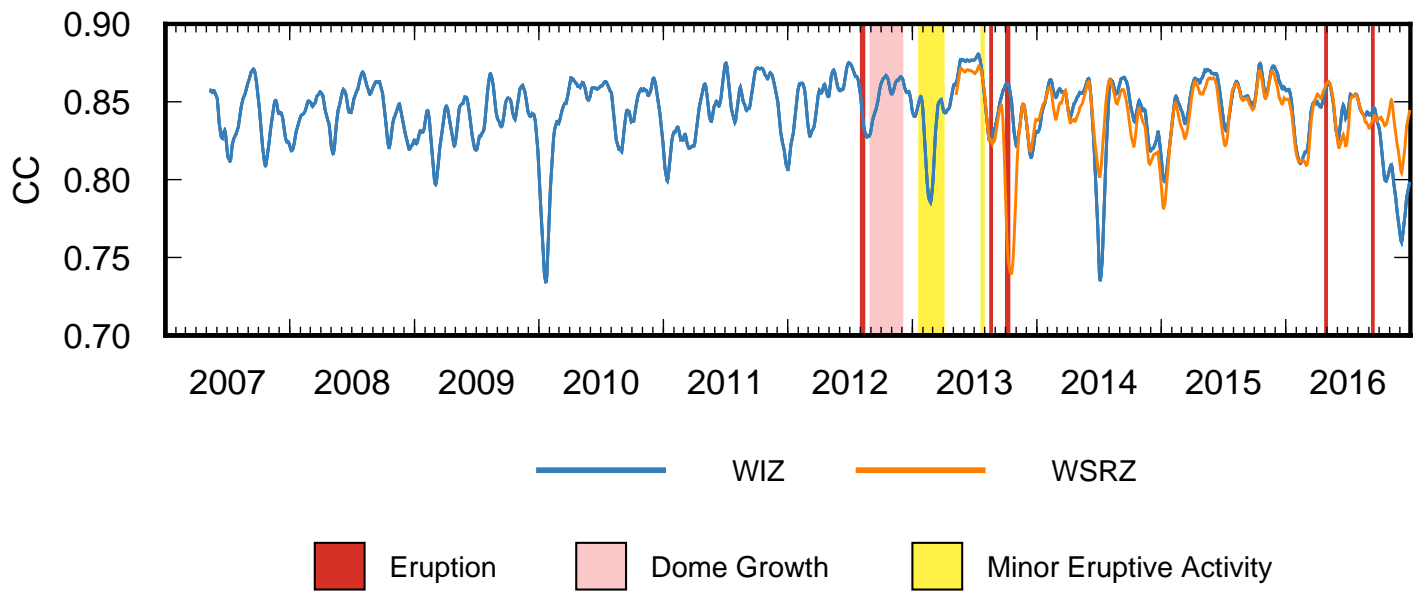

Figure 3.18: Velocity changes and correlation coefficient measurements for WIZ and WSRZ time series, smoothed with a 14-day centered moving window. (a) Velocity changes for WIZ. (b) Velocity changes for (c) Average correlation coefficient for WIZ and WSRZ time series.

The introduction of WSRZ station in 2013 provides an additional measure of velocity changes during late-2013 and 2016 eruptive activity. Amplitudes of velocities for WSRZ are similar to those for WIZ, varying between approximately $\pm 0.02 \%$, but otherwise show considerable differences in short-term velocity changes (Fig. 3.18b). Correlation coefficient measurements are slightly more consistent between the two time series, with only three periods where they differ significantly (Fig. 3.18c). The first occurs just before and during the October 2013 eruption sequence, where the correlation coefficient for WSRZ reaches its lowest point over the four-year period. The next occurs mid-2014, with the reversed scenario of a reduced correlation co- 


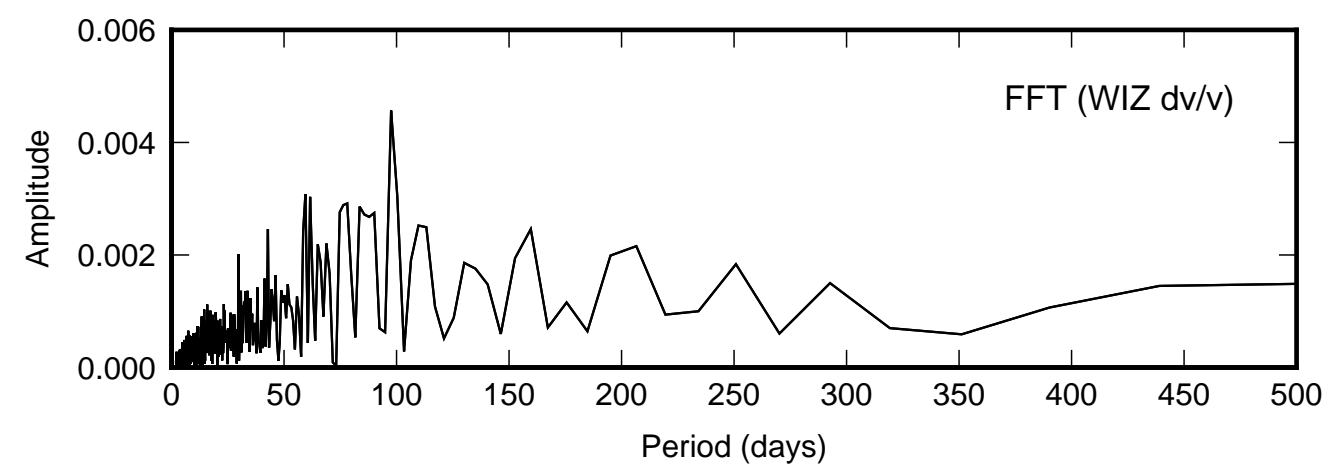

Figure 3.19: Fast Fourier Transform of velocity changes (dv/v) for WIZ station.

efficient for WIZ. The final deviation between both stations occurs after activity in September 2016 where, unlike WIZ station, the correlation coefficient does not appear to decline in the following months. Velocities recorded for WSRZ do however appear to decline during this period, with velocities $0.02 \%$ lower by the end of 2016 than pre-September levels (Fig. 3.18b).

Velocity changes in Figure 3.18 appear to show strong systematic fluctuations. The most obvious of these occur in 2007-2008, where velocities alternate between a positive and negative change at a similar rate (Figure 3.18a). We perform a Fast Fourier Transform (FFT) on WIZ velocity changes to investigate possible systematic changes, after first demeaning, detrending and applying a 5\% cosine taper to raw, non-smoothed, velocity changes (3.19). Velocities are shown to be dominated by long-period signals, with many peaks above a period of approximately 60 days. The strongest of these is found at approximately 100-days.

Long-term behaviour of velocities is revealed in Figure 3.20 by smoothing data with a 180-day window. The resultant trend captures decreasing velocities in 2007-2008, with velocities then increasing through to early 2012. The rate of this increase is fastest in the year following the mid-2008 velocity low. From 2012-2016, long-term changes fluctuate between velocity highs and lows on an approximate two-year period. The first velocity low covers all eruptive activity in 2012-2013. The second occurs early-to-mid 2015, during a period of large short-term velocity changes. In 2016, the long-term trend is dominated by the rapid velocity decrease in September 2016. The short time-scale of this change has introduced an artificial time shift, with the long-term trend starting to decline in early 2016. 


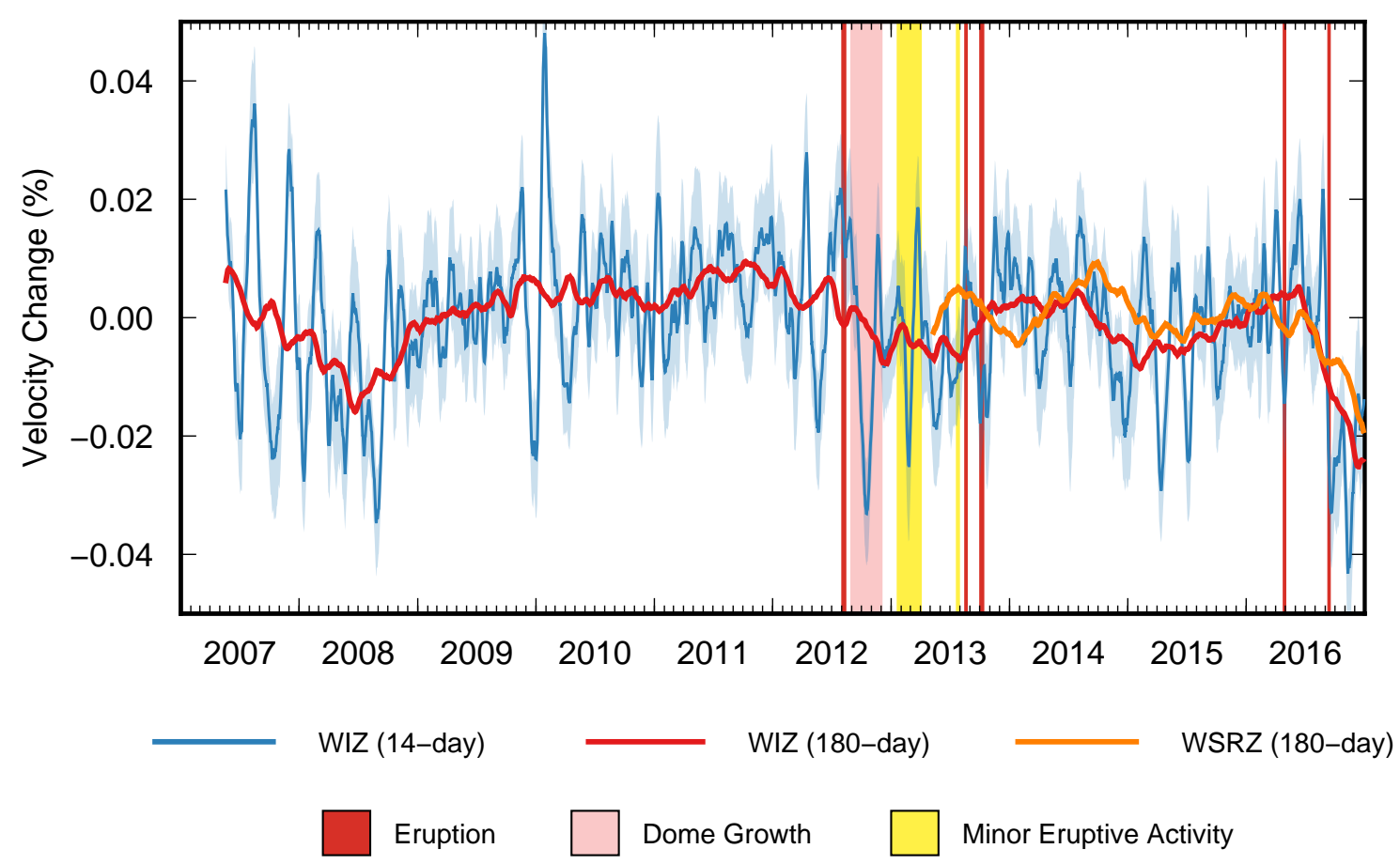

Figure 3.20: Long-term trend of velocity changes for WIZ and WSRZ stations using a 180-day centered moving-window. Also shown are 14-day-smoothed WIZ velocity changes. Note the transient decline in velocity following the September 2016 eruption results in a long-term trend that artificially begins declining earlier.

\subsubsection{Stability of Measurements}

Velocity changes around White Island are sought through averaging multiple stationpairs involving a single seismic station on the volcano. This aims to average out velocity changes originating away from the volcano. In this section, we look at the stability of the average velocity change by removing individual station-pairs from the dataset prior to computation. Velocity changes with origins close to the volcano are expected to remain in the average no matter which station-pair is removed. Conversely, velocity changes originating away from the volcano are more likely to be recovered by fewer station-pairs. Note though that this process does not effectively distinguish between local and regional changes, which could be detected by all station-pairs.

Four separate years of data are shown in Figure 3.21 after recomputing the average velocity change for WIZ station with a different station-pair removed each time. For all station-pairs other than URZ-WIZ - with only vertical component data downloaded from URZ - this means removing nine time series from the average, corresponding to the different component-pairs. Conversely, only three time series are removed when excluding URZ-WIZ (ZR, ZT and ZZ). The coherency between 
the five different averages - one for each station removed - is observed to be variable (Figure 3.21). For example, the average is relatively stable in the weeks before the September 2016 eruption compared to velocity changes in the months following (Fig. 3.21d). Similarly, velocity changes are stable in the $2-3$ months before the October 2013 eruption, becoming more variable immediately after (Fig. 3.21c). We further explore this by plotting the variance of the five averages from 2007-2017 in Figure 3.22. Larger variances are recorded from 2007-2011 relative to variances calculated between 2012-2017. This may indicate an increased dominance of velocity changes at White Island relative to background fluctuations in the Bay of Plenty region. Note, however, that station-pair HAZ-WIZ became active in January 2010. This could account for some of this difference, though relatively high variance is still observed until mid-2011 despite the introduction of HAZ station.

Finally, we show the effect of removing individual station-pairs on the long-term

(a)

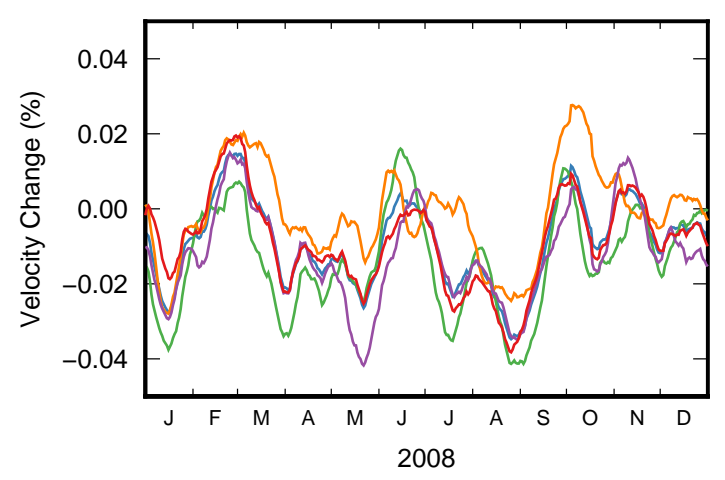

(c)
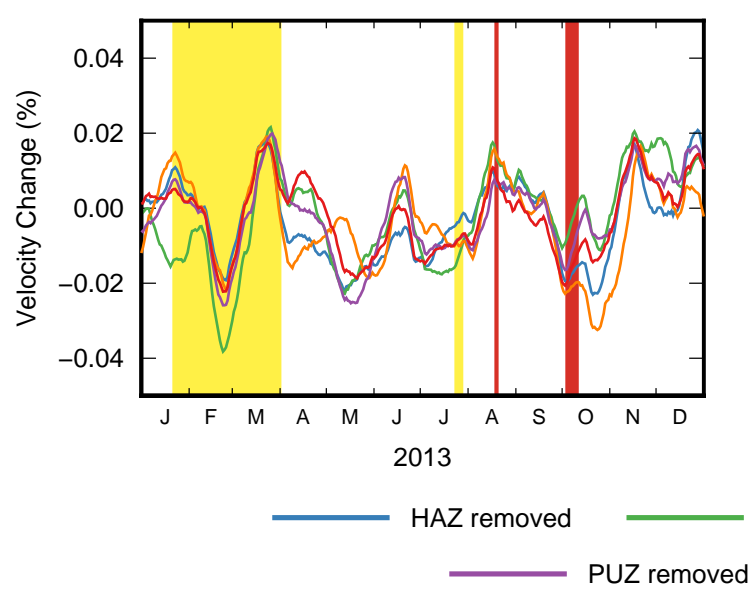

Eruption (b)

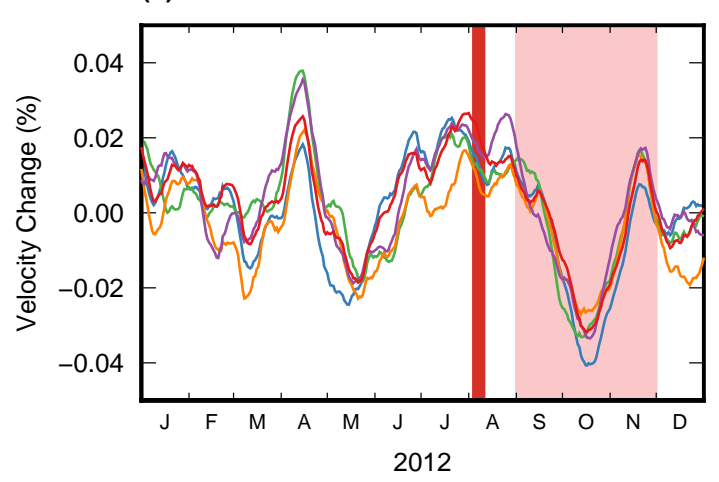

(d)

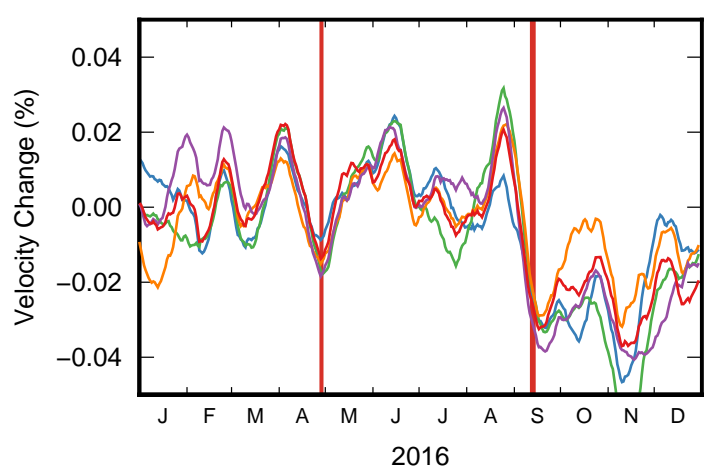

OPRZ removed

Minor Eruptive Activity

Figure 3.21: Plots of WIZ averaged time series with a different station-pair removed each time. This gives a total of five different averages, all smoothed using a 14-day moving window. Each subplot corresponds to velocity changes in: (a) 2008, (b) 2012, (c) 2013, (d) 2016. 


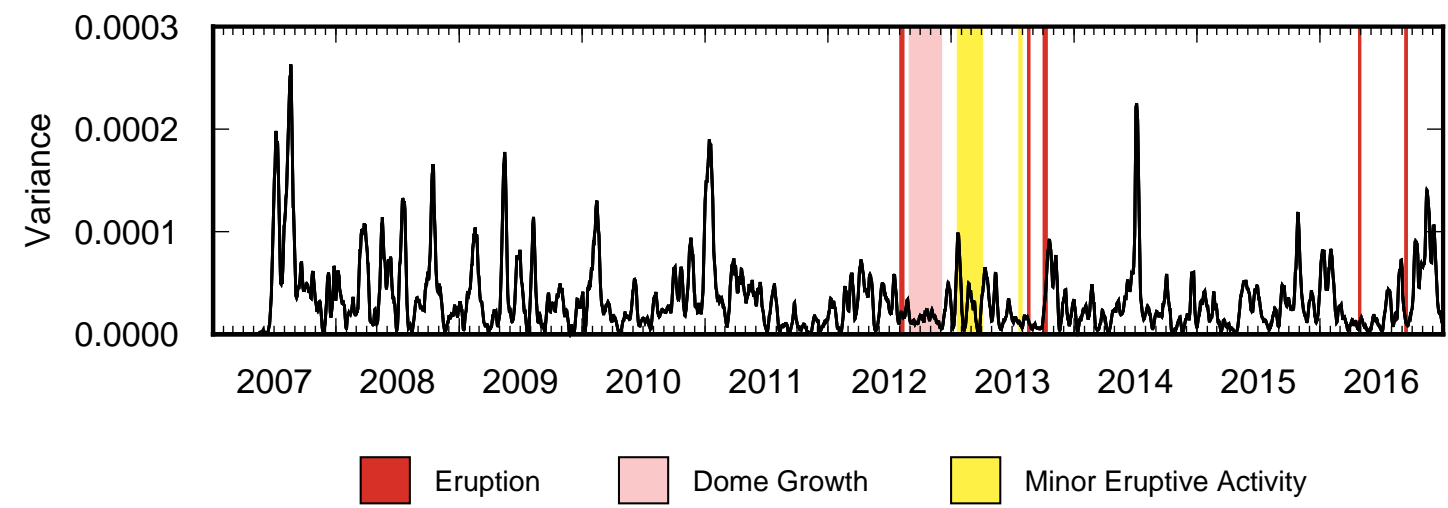

Figure 3.22: The variance of the different WIZ averaged time series minus one stationpair is shown with time, illustrating changes in the stability of velocity measurements.

average for WIZ (Fig. 3.23). Significant changes in the average occur when the station-pair OPRZ-WIZ is removed. For example, the velocity low in 2008 is not as clearly recovered without this station-pair. Additionally, a velocity low is observed in 2010-2011 that is not present when OPRZ station is included. This suggests a dominant influence of velocities recorded by OPRZ-WIZ in the average. From 2012 however, the long-term trend is much more coherent between the five averages, with the velocity decrease during activity in 2012-2013, and subsequent recovery by mid2014, not dependent on any one station. Long-term trends during this period are therefore the most likely candidates for velocity changes originating at White Island volcano. 


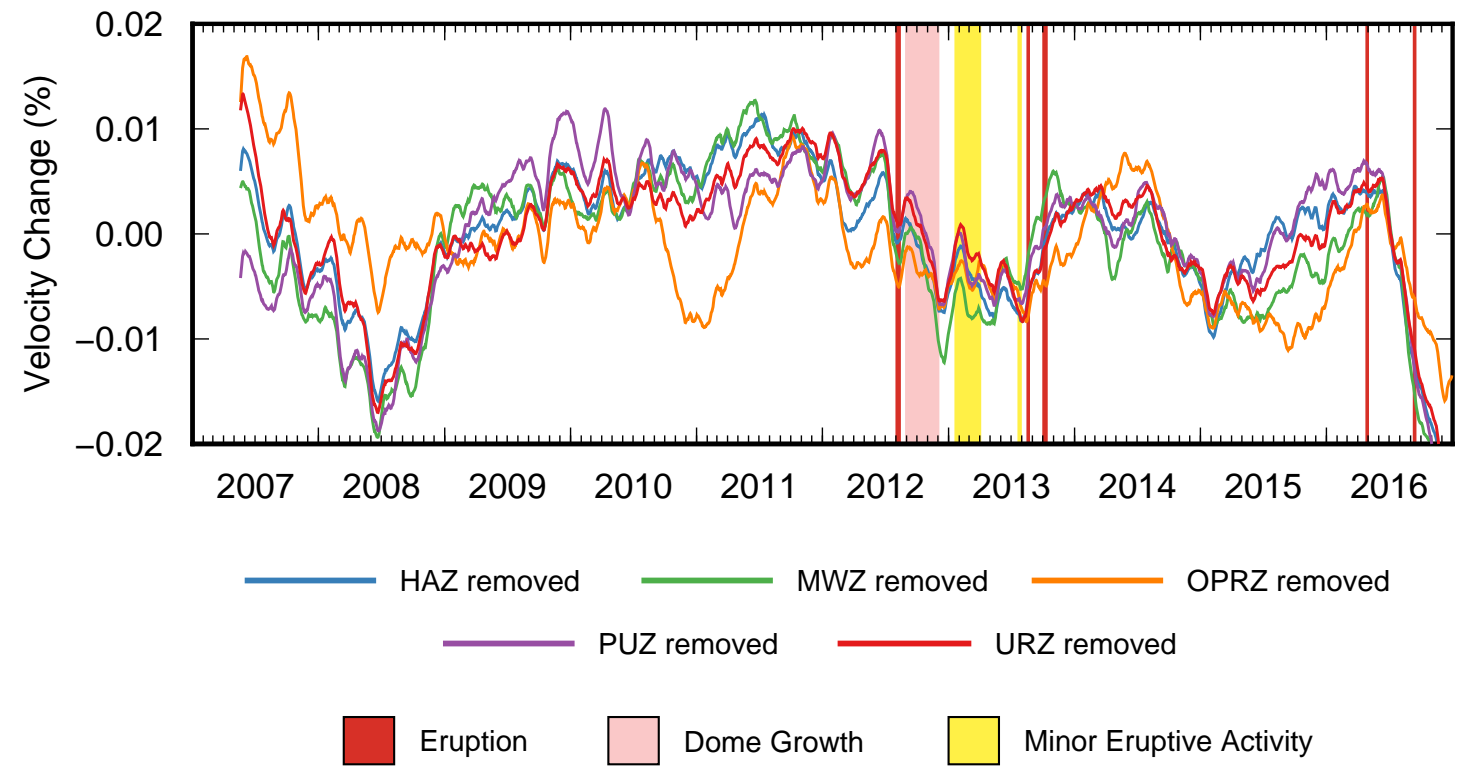

Figure 3.23: Plot of the long-term trend at WIZ (180-day smooth), recomputed five times with a different station-pair removed each time. Chosen pair is denoted by the key, noting that all paths used involve an onshore station (listed in key) and WIZ. 


\section{Chapter 4}

\section{Single-Station Dataset}

This chapter focusses on the processing and results of the single-station ambient noise dataset. This includes stations located on the volcano (offshore) in addition to stations located onshore for comparative purposes (Section 4.3.2). Parameter justification in Section 4.2 draws upon methodology used in Chapter 3 and therefore does not include full descriptions here. The chapter also looks at velocity changes in the context of nearby tectonic earthquakes (Section 4.3.3).

\subsection{Introduction}

The single-station dataset includes two seismic stations located on White Island volcano (WIZ and WSRZ, Fig. 1.2). Processing follows the single-station approach to ambient noise monitoring, correlating components of a single station with themselves to produce auto-correlations (AC) or with each other to give single-station cross-component correlations (SC). This is advantageous at White Island with only one permanent station running throughout eruptive activity in 2012-2016. We also process four onshore stations using the single-station approach. This allows us to distinguish between local and regional velocity changes, through comparison with stations on White Island. The four stations chosen for this correspond to the onshore stations used in the final results of Chapter 3 (HAZ, MWZ, OPRZ and PUZ), with URZ station excluded due to only vertical component data being acquired at this site.

\subsection{Parameter Justification}

In this section, we consider the different parameter choices available within MSNoise just as we did in Section 3.2. Some steps performed in Chapter 3 are excluded here, either because they are no longer valid for single-station processing or because of time limitations. For example, with no direct arrivals in single-station data, com- 
puting the spectral SNR - as done in Chapter 3 (Section 3.2.1) - is no longer valid. We therefore process data between frequency bounds $0.1-1.0 \mathrm{~Hz}$ and $1.0-2.0$ $\mathrm{Hz}$ without further testing. These frequencies are similar to those used in previous single-station noise studies (e.g. Hobiger et al., 2014; De Plaen et al., 2016). Additionally, no synthetic testing is performed to determine parameters for the moving-window cross-spectral technique.

Parameter testing starts with an initial one month sample dataset and iteratively compares the different parameter choices, as performed in Chapter 3. This sample dataset includes all components at WIZ station from 1 June 2010 to 1 July 2010. Cross-correlation functions are computed by applying a bandpass filter at both $0.1-$ $1.0 \mathrm{~Hz}$ and $1.0-2.0 \mathrm{~Hz}$, such that parameter testing is performed for both frequency ranges individually. This aims to determine if the ideal parameter choices vary depending on the frequency of noise sources. Data are then 1-bit normalised and whitened between $0.12-0.98$ or $1.02-1.98 \mathrm{~Hz}$ depending on the initial bandpass filter applied. Recall that whitening is only applied to the cross-components (Section 2.3.2). Finally, the data are resampled to $10 \mathrm{~Hz}$ and cross-correlations are computed between 30 minute time slices and a linear stack performed. These choices then act as our initial parameter set that are iteratively modified in the next section to maximise the SNR. The reader is referred to Appendix A.2 for final parameter choices following this section.

\subsubsection{Maximising Temporal Stability}

This section aims to maximise the SNR of the initial dataset - as performed in Chapter 3 (Section 3.2.2) - by iteratively varying the pre-processing parameters and recomputing the SNR of resultant cross-correlation functions. Choices available include whether to perform 1-bit normalisation or clip the data at three times the root-mean-squared amplitude, change the length of time slices to correlate, and using a linear or phase-weighted stack. We exclude lag times between -7.5 and 7.5 seconds from SNR computation when correlating components of a single seismic station, in order to avoid strong peaks at zero-time.

The maximum SNR was obtained for both $0.1-1.0 \mathrm{~Hz}$ and 1.0-2.0 Hz datasets by clipping amplitudes at three times the root-mean square amplitude and performing a linear stack (Fig. 4.1 and 4.2). For 0.1-1.0 Hz cross-correlations, improvements in the SNR are observed at all lag times after applying these steps (Fig. 4.1a and 4.1c). For 1.0-2.0 Hz cross-correlations however, improvements were limited to lag times less than approximately 40 seconds (Fig. 4.2a and 4.2c). These findings clearly indicate that clipping amplitudes based on the root-mean-squared amplitude and 
linear stacking are preferable for White Island ambient noise monitoring, given similar findings in Chapter 3 when processing the station-pair dataset (Fig. 3.4).

Different choices of correlation length are chosen for the different frequency bands. For $0.1-1.0 \mathrm{~Hz}$ cross-correlations, the choice of correlation length has minimal influence on the SNR (Fig. 4.1b). A final correlation length of 7200 seconds (2 hours) is chosen for this dataset owing to marginal improvements. For 1.0-2.0 Hz crosscorrelations however, increasing the correlation length improves the SNR at later lag times (Fig. 4.2b). We investigate this further in Appendix A.4, finding that the majority of this improvement is accounted for in the cross-components. We there-
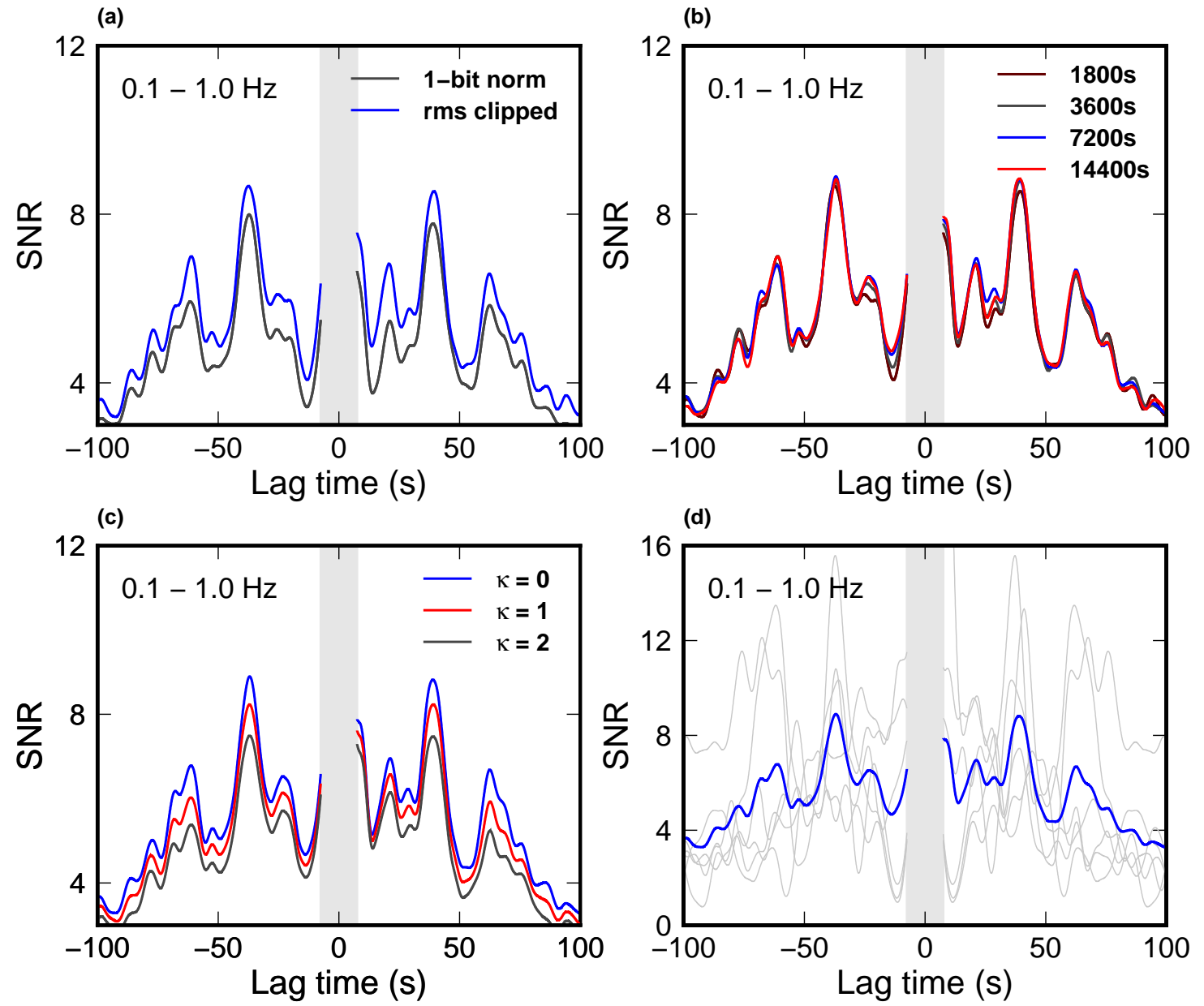

Figure 4.1: Different parameter choices are tested to maximise the average SNR of cross-correlation functions in the sample dataset filtered between $0.1-1.0 \mathrm{~Hz}$. The SNR is greyed out between -7.5 and 7.5 seconds lag time, to avoid peaks at zero lag time. (a) 1-bit normalisation vs. clipping at 3 times the RMS. (b) Varied correlation lengths, after performing rms-clipping. (c) Varied power $v$ for the phase-weighted stack, after rms-clipping and correlating in 7200 second time slices. (d) Final maximised SNR is shown in blue, with the SNR of individual component-pairs shown in grey. 
(a)

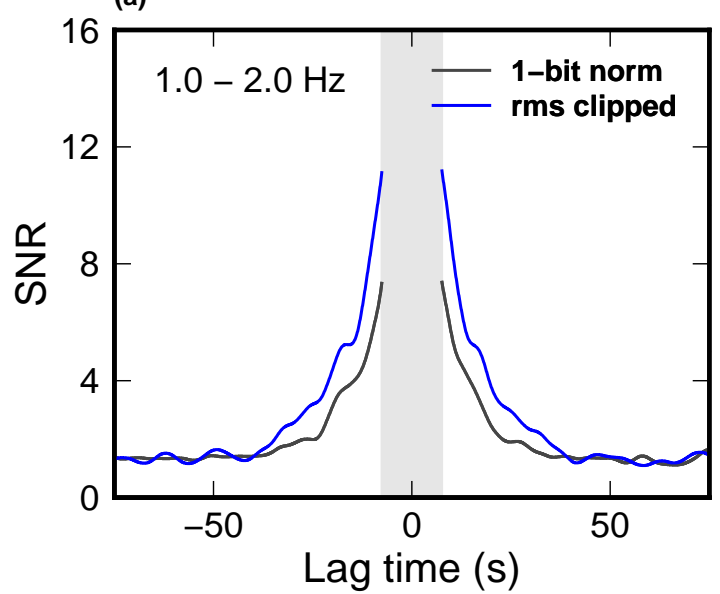

(c)

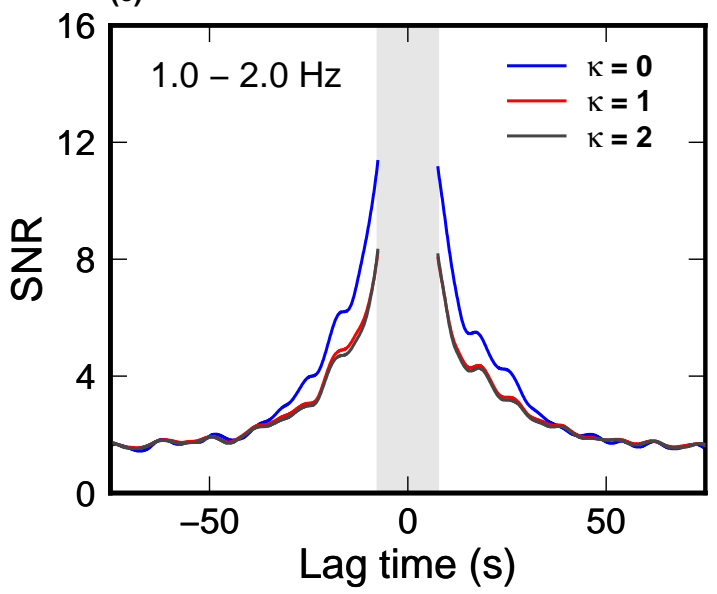

(b)

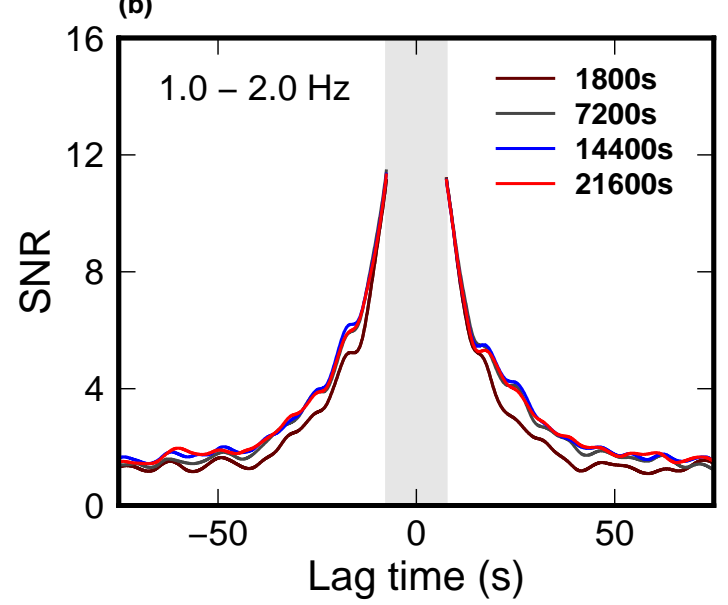

(d)

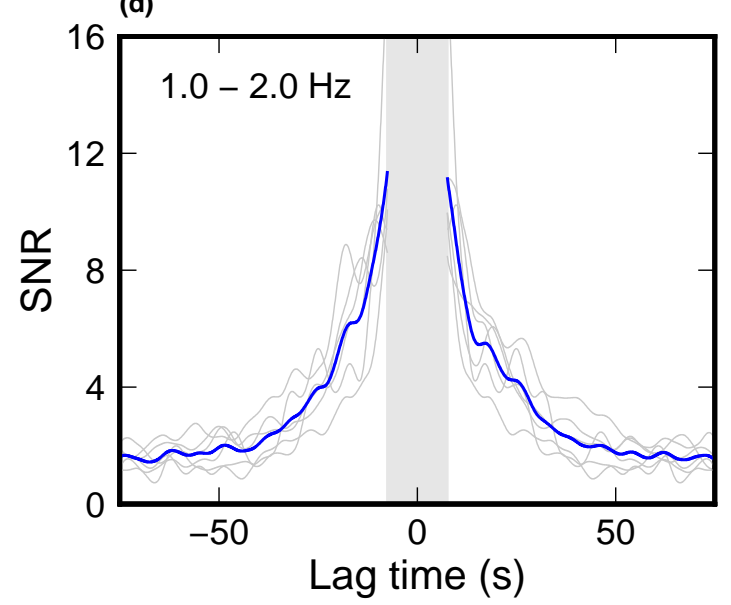

Figure 4.2: Different parameter choices are tested to maximise the average SNR of cross-correlation functions in the sample dataset filtered between 1-2 Hz. The SNR is greyed out between $\mathbf{- 7 . 5}$ and $\mathbf{7 . 5}$ seconds lag time, to avoid peaks at zero lag time. (a) 1-bit normalisation vs. clipping at 3 times the RMS. (b) Varied correlation lengths, after performing rms-clipping. (c) Varied power $\kappa$ for the phase-weighted stack, after rms-clipping and correlating in 14400 second time slices. (d) Final maximised SNR is shown in blue, with the SNR of individual component-pairs shown in grey.

fore cross-correlate 1.0-2.0 Hz noise data using 14,400 second (4 hour) time slices, after checking that increases in the SNR reflect real improvements (Fig. 4.1 and A.5).

\subsubsection{Stack-size Analysis}

The stack size required to produce high correlation coefficient measurements is observed to be lower for single-station ambient noise than was required for the stationpair dataset (Fig. 4.3). We compare 1-day and 30-day stacks over two years for both 0.1-1.0 Hz and 1.0-2.0 Hz datasets (Fig. 4.3a and 4.3b respectively). The average correlation coefficient - averaged over all auto-correlations and cross-components — is found to be similar between 1-day and 30-day stacks in both cases, with only 
small improvements in the 30-day stack. This is likely to be related to the distance covered by scattered energy — rather than a characteristic of single-station datasets - with smaller distances requiring less stacking to resolve stable crosscorrelation functions. To check this, we examine correlation coefficients of 1.0-2.0 $\mathrm{Hz}$ cross-correlation functions computed for the station-pair WIZ-WSRZ, using the same parameters as used for 1.0-2.0 Hz single-station processing. Similar results are found, with only small improvements observed after increasing the stack size to 30-days (Fig. 4.3c). This is in contrast to large differences in the average $\mathrm{CC}$ found between 1-day and 30-day stacks in Chapter 3 (Fig. 3.8). This suggests that single-station datasets require less stacking relative to the station-pair dataset (Chapter 3), due to smaller distances that energy has to travel. Going forward, we use 10-day stacks to process single-station ambient noise, which should be sufficient
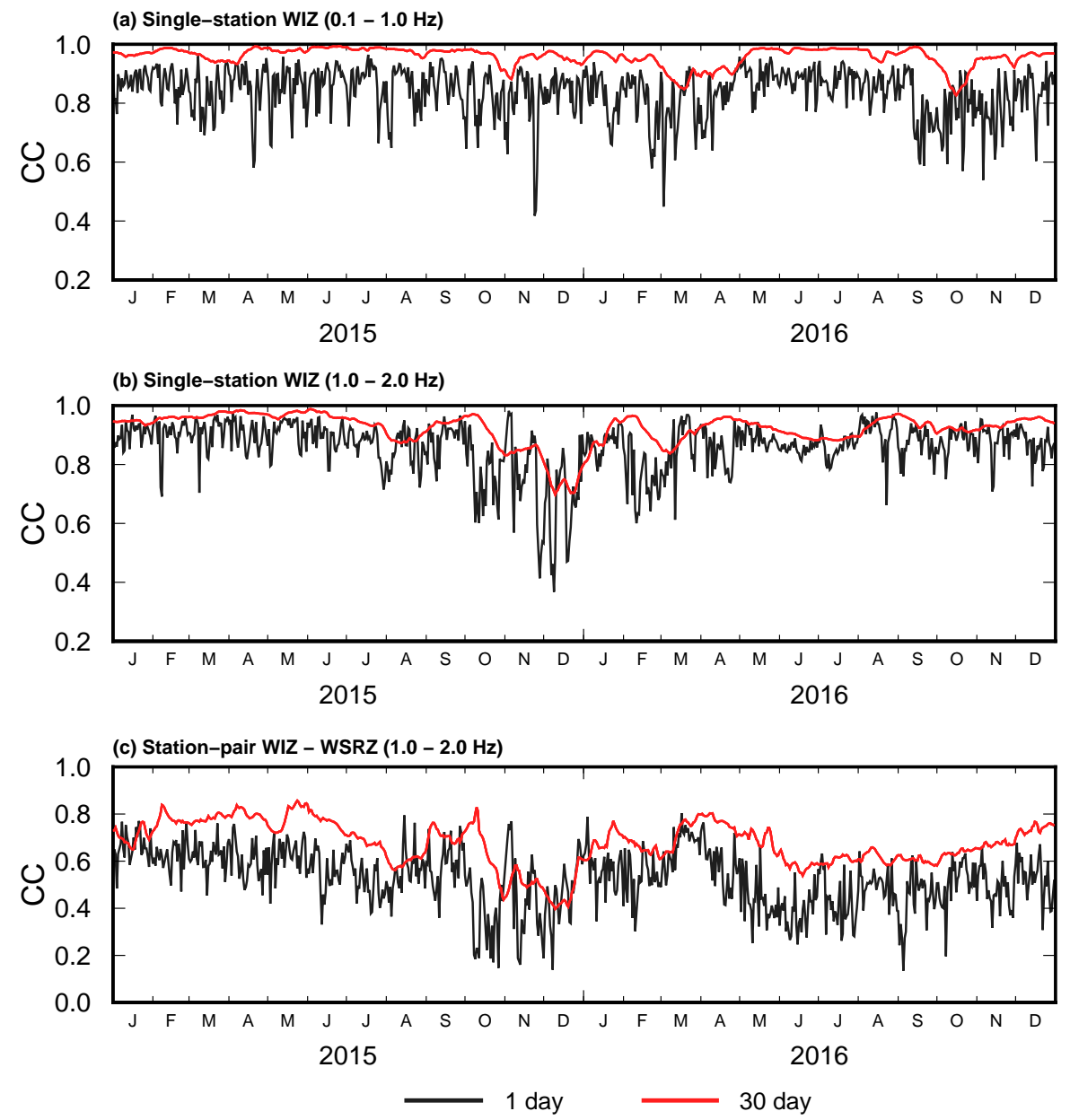

Figure 4.3: Comparison between correlation coefficients calculated for 1 day stacks (black line) and 30 day stacks (red line). The correlation coefficient is calculated between current and reference stacks, with an average shown across all componentpairs. (a) $0.1-1.0 \mathrm{~Hz}$ single-station correlations (WIZ). (b) 1.0-2.0 Hz single-station correlations (WIZ). (c) 1.0-2.0 Hz station-pair correlations (WIZ-WSRZ). This then encompasses nine different component-pairs instead of six. 
to record accurate velocity changes. Note that we process the station-pair WIZWSRZ through to velocity changes also, but do not include this in our main results. Instead, this dataset can be found in Appendix B.2.

\subsubsection{SNR vs Coherence}

In Chapter 3 we plotted the SNR against coherence to determine what a threshold SNR for measuring accurate velocity changes. This helped to define the minimum and maximum lag times from which reliable delay time measurements — calculated during the moving-window cross-spectral techique - could be made. We repeat this here for single-station datasets, using a 16 second and a 12 second window length for the moving-window cross-spectral technique for $0.1-1.0 \mathrm{~Hz}$ and $1.0-2.0 \mathrm{~Hz}$ data respectively. While we do not perform further synthetic testing to determine these, we consider these window lengths appropriate given results from Chapter 3 . This found that larger window sizes were better suited to recovering accurate velocity changes (Section 3.2.3). Additionally, we set the step-size between successive windows to four seconds for both frequency ranges. Finally, reference stacks are produced using all available data from 2007-2017. The final relationship between the SNR and coherence is produced by recording the value of each of these at different lag times.

Figure 4.4 compares the SNR against coherence for 10-day stacks of $0.1-1.0 \mathrm{~Hz}$ and 1.0-2.0 Hz cross-correlation functions. Auto-correlations and cross-components are plotted individually to highlight the differences between the two datasets. The relationship between the SNR and coherence for the cross-components follows a similar exponential relationship to that of Chapter 3, with high values of coherence above a SNR of approximately 2 (Fig. $4.4 \mathrm{~b}$ and $4.4 \mathrm{~d}$ ). Below this, the coherence starts to fall more rapidly. There is some evidence of a deviation from an exponential relationship, with more rapid increases in coherence at higher values of SNR observed in 1.0-2.0 Hz cross-components (Fig. 4.4d). This relationship is not evaluated further however, as it is not important in terms of defining a threshold on the minimum SNR.

The relationship between the SNR and coherence is less clear for auto-correlations. For $0.1-1.0 \mathrm{~Hz}$ auto-correlations (Fig. 4.4a), there is little evidence that a higher SNR results in improved waveform coherence. We note though that the range of SNR values recorded for this dataset is small, with no values recorded below a SNR of 2 and none above a SNR of 6 . A small SNR range may therefore be responsible for the lack of a clearly observed exponential trend. For $1.0-2.0 \mathrm{~Hz}$ auto-correlations, an exponential relationship is partially resolved; though, we find that the majority of measurements made at various lag times sit below a SNR of 2. This suggests that 1.0-2.0 Hz auto-correlations could suffer from reduced temporal stability. 
(a)

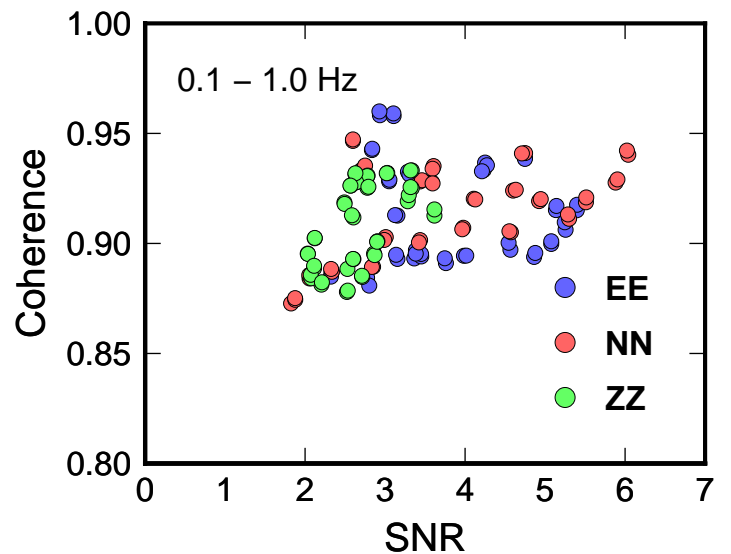

(c)

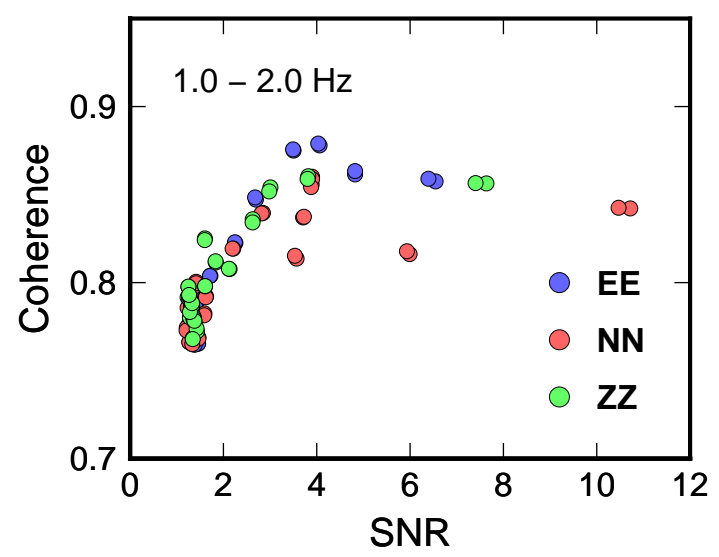

(b)

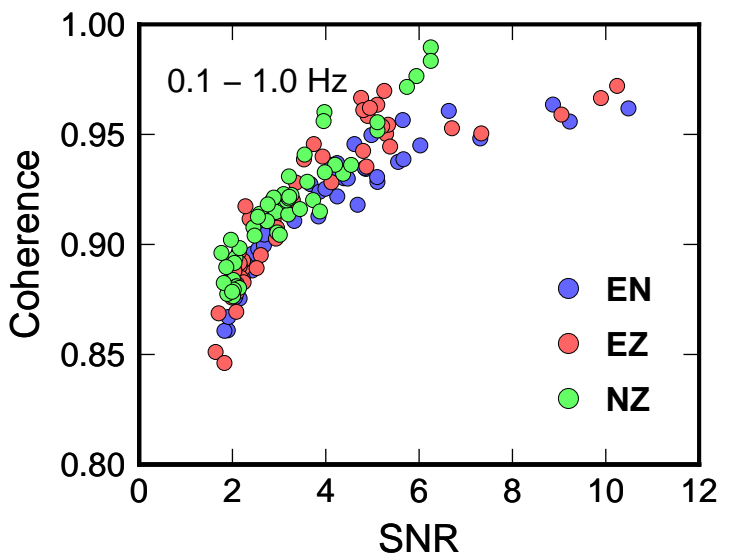

(d)

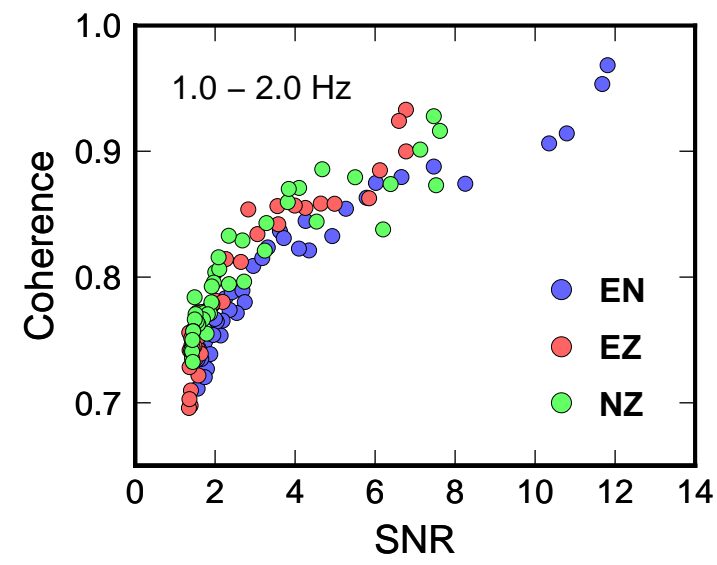

Figure 4.4: SNR versus the coherence plotted individually for auto-correlations (AC) and cross-components (SC) for both $0.1-1.0 \mathrm{~Hz}$ and $1.0-2.0 \mathrm{~Hz}$ correlations. (a) 0.1-1.0 Hz, AC. (b) $0.1-1.0 \mathrm{~Hz}$, SC. (c) $1.0-2.0 \mathrm{~Hz}$, AC. (d) $1.0-2.0 \mathrm{~Hz}, \mathrm{SC}$.

Minimum and maximum lag times for velocity analysis are chosen in a similar manner to Chapter 3 (Section 3.2.5), only differing in how the minimum lag time is chosen. This is set to avoid energy at near-zero lag times, rather than excluding direct arrivals associated with station-pairs. The minimum lag is therefore set to 20 seconds for $0.1-1.0 \mathrm{~Hz}$ data and 10 seconds for $1.0-2.0 \mathrm{~Hz}$ data. The maximum lag time is again chosen to exclude data below a SNR of 2. In Figure 4.5, the average SNR of 10-day stacks - recorded over one year - is shown for both autocorrelations and cross-components individually. We do not modify MSNoise scripts this time to allow for individual choices of the maximum lag for auto-correlations and cross-components. Thus, for the same frequency band, both auto-correlations and cross-components require the same choice of minimum and maximum lag. We choose a maximum lag time of 80 seconds for $0.1-1.0 \mathrm{~Hz}$ data and a maximum lag time of 40 seconds for $1.0-2.0 \mathrm{~Hz}$ data. This corresponds to the approximate lag time that the SNR falls below 2 for both datasets (Fig. 4.5). Note that the SNR of 0.1-1.0 Hz auto-correlations does not fall below this threshold within \pm 100 seconds 
(a)

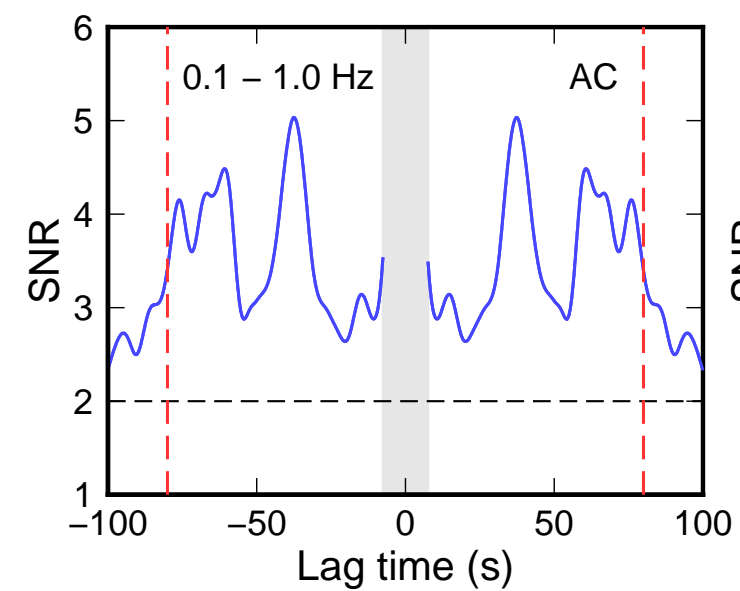

(c)

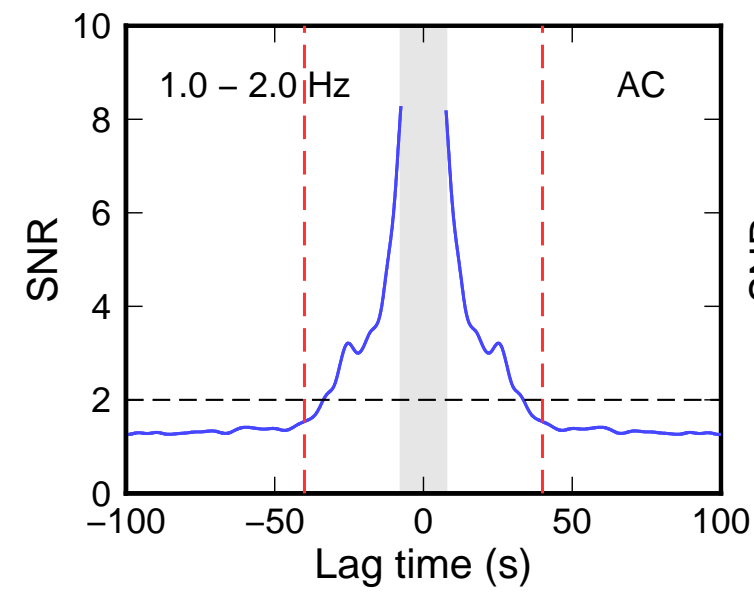

(b)

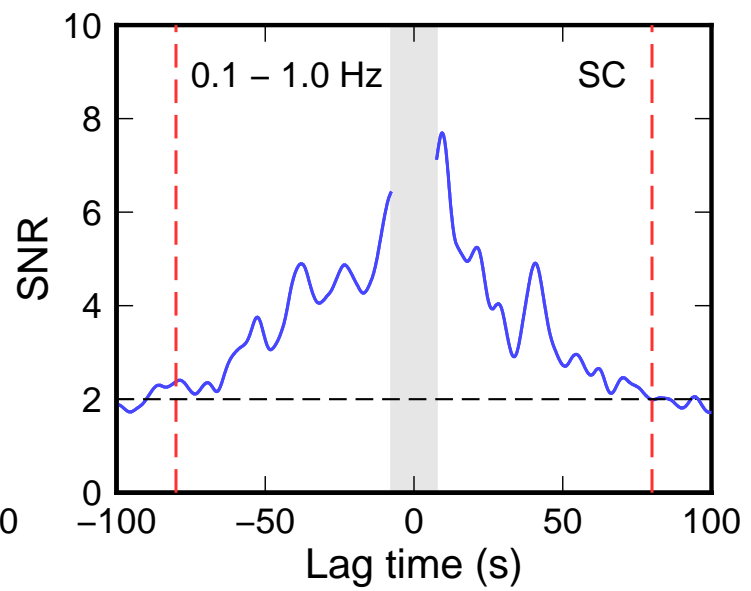

(d)

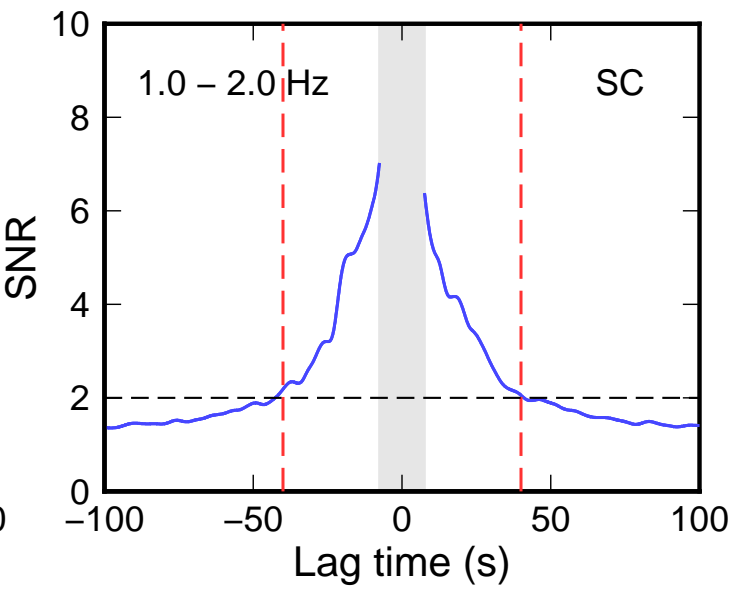

Figure 4.5: Average SNR of 10-day stacks from 1 June 2010 to 1 June 2011 for autocorrelations (AC) and cross-components (SC) individually for both $0.1-1.0 \mathrm{~Hz}$ and 1.0-2.0 $\mathrm{Hz}$ correlations. A threshold SNR value of 2 is indicated by the horizontal dashed black line, and the chosen maximum lag time indicated by the vertical dashed red line. (a) $0.1-1.0 \mathrm{~Hz}$, AC. (b) $0.1-1.0 \mathrm{~Hz}$, SC. (c) $1.0-2.0 \mathrm{~Hz}$, AC. (d) $1.0-2.0 \mathrm{~Hz}$, SC.

lag time (Fig. 4.5a), but is set to 80 seconds to accomodate the reduced SNR of the cross-components at later lag times (Fig. 4.5b). Additionally, this captures a period of higher SNR in the auto-correlations from approximately 50-80 seconds at both positive and negative lags (Fig. 4.5a).

\subsubsection{Final Parameter Choices for Velocity Analysis}

Final parameter choices, relating to quality control, are chosen in a similar manner to those in Chapter 3. Choices include the maximum accepted delay time, maximum error and minimum coherence of delay time measurements used in performing the final weighted linear regression to resolve a velocity change. The maximum delay 
time is calculated based on a maximum expected velocity change of $0.5 \%$, following Equation 3.6. This corresponds to a maximum delay time 0.4 seconds for $0.1-1.0 \mathrm{~Hz}$ measurements and 0.2 seconds for 1.0-2.0 Hz measurements. As before (Chapter 3), the maximum error is set to 0.1 seconds. The choice of $0.5 \%$ is again based on the magnitudes of velocity changes typically observed by global ambient noise studies at volcanoes (e.g. Brenguier et al., 2008b; Obermann et al., 2013; Bennington et al., 2015). It might be expected that single-station monitoring on the volcano could detect changes above $0.5 \%$, because of a closer proximity to the volcano. Note though that the maximum delay time is chosen here based on the expected delay at the maximum lag time. Therefore, a larger velocity change should still be detectable within quality control criteria at earlier lag times. For example, a 1.0\% velocity change detected by $0.1-1.0 \mathrm{~Hz}$ ambient noise would still produce delay times within the quality control criteria between 20 and 40 seconds.

The minimum coherence for each frequency band is chosen by examining the values of coherence recorded in Figure 4.4. For 0.1-1.0 Hz measurements, a SNR of 2 corresponds to a coherence of approximately 0.85 (Fig. 4.4a and 4.4b). We therefore choose a minimum coherence of 0.8 for $0.1-1.0 \mathrm{~Hz}$ velocity changes which, while slightly conservative, still represents a high value of coherence. Similarly, for 1.0$2.0 \mathrm{~Hz}$ measurements a SNR of 2 corresponds to a coherence of approximately 0.7 . We therefore choose a minimum coherence of 0.6 for $1.0-2.0 \mathrm{~Hz}$ velocity changes. Once again, the reader is referred to Appendix A.2 for a complete list of the final parameters chosen in this thesis.

\subsection{Results}

This section presents the velocity changes measured from single-station ambient noise processing, starting with those recorded by White Island stations (Section 4.3.1). We then compare these results to velocity changes recorded by onshore stations (Section 4.3.2) and, as a result of findings in this section, investigate the possibility of tectonic earthquake induced changes (Section 4.3.3).

Results are presented for auto-correlations and cross-component correlations individually within the frequency bands $0.1-1.0 \mathrm{~Hz}$ and $1.0-2.0 \mathrm{~Hz}$. For the autocorrelations, we average velocity changes recorded by components EE, NN and ZZ. For the cross-components, we average velocity changes recorded by components EN, EZ and NZ. A weighted-arithmetic mean is used to average these components, following the same approach described in Section 3.3.1. 


\subsubsection{Velocity Changes at White Island}

Velocity changes recorded by WIZ and WSRZ stations are presented first for 0.1-1.0 $\mathrm{Hz}$ data followed by $1.0-2.0 \mathrm{~Hz}$ data. As in Chapter 3, we also measure the correlation coefficient between current and reference stacks within defined minimum and maximum lag times. Only the final averaged time series for auto-correlations and cross-components are shown, with velocity changes for individual component-pairs found in Appendix B.1.

Short-term systematic fluctuations in velocity were observed in single-station results, potentially concealing more important changes. These were particularly dominant in $0.1-1.0 \mathrm{~Hz}$ auto-correlations, with a Fast-Fourier transform revealing a dominant period of approximately 30 days (Fig. 4.6a). A 30-day moving-window smooth is applied to velocities to average out these short-term fluctuations, reducing their influence on the final time series. We acknowledge that some temporal resolution will be lost during smoothing, though identify no obvious short-term changes above background levels that suggest significant results have been excluded.

Longer period peaks are also observed in the amplitude spectrum of velocity changes at WIZ station that could be indicative of a seasonal trend (Fig. 4.6). In all datasets shown in Figure 4.6, a strong peak is observed between 300-400 days period, with this the dominant period in $0.1-1.0 \mathrm{~Hz}$ cross-components and 1.0-2.0 Hz auto-correlations (Fig. 4.6b and 4.6c). These observations suggest a seasonal signal

(a)

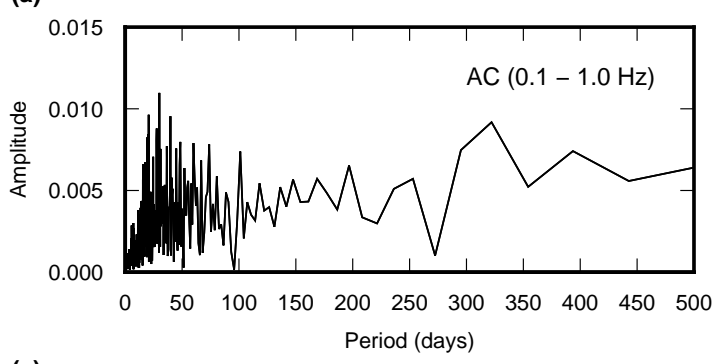

(c)

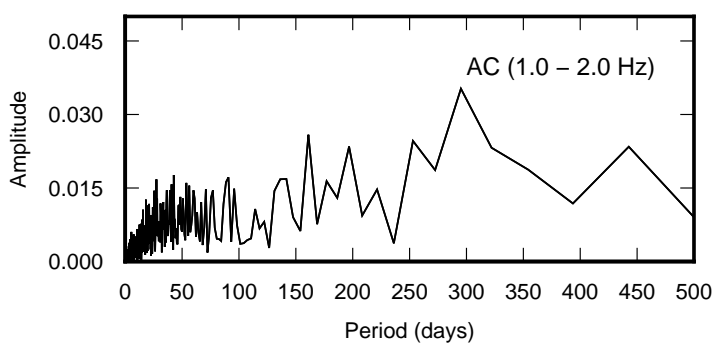

(b)

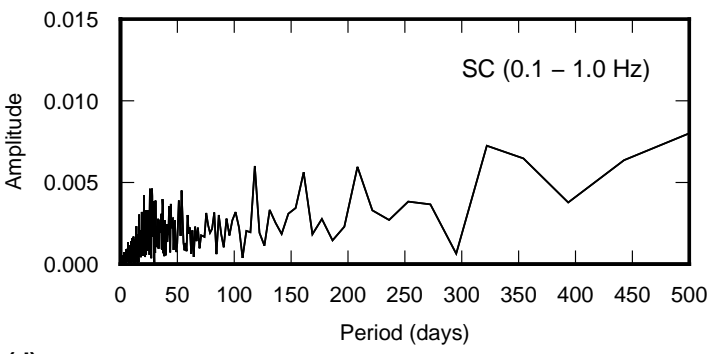

(d)

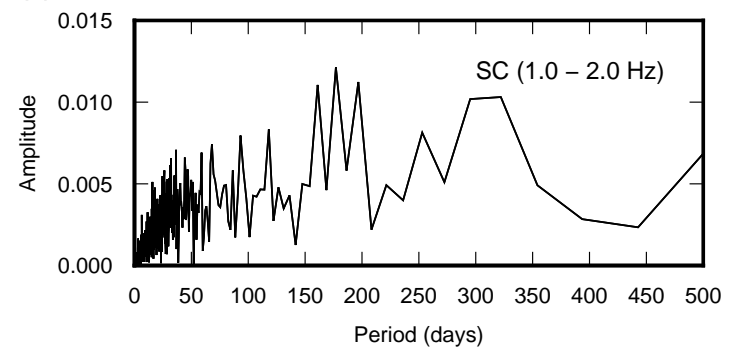

Figure 4.6: Fast Fourier Transform of velocity changes measured for WIZ station. (a) Auto-correlations, 0.1-1.0 Hz. (b) Cross-components, 0.1-1.0 Hz. (c) Autocorrelations, 1.0-2.0 Hz. (d) Cross-components, 1.0-2.0 Hz. 
could be present at White Island volcano, with implications for interpreting velocity changes as either volcanic or non-volcanic.

Results from $0.1-1.0 \mathrm{~Hz}$ auto-correlations and cross-components are shown in Figure 4.7. One of the strongest features is an apparent long-term velocity increase from 2008 to the April 2016 eruption, best revealed by the cross-components recorded at WIZ station (Fig. 4.7c). The start of this trend coincides with a sharp velocity decrease of approximately $0.1 \%$ in mid-2008 (Fig. 4.7c). A decrease in velocity is also detected by auto-correlations during this period, though is spread over $4-5$ months (Fig. 4.7a). Over the next eight years long-term velocities at WIZ station increase by approximately $0.15 \%$, peaking in early-2016. In the cross-component changes, this peak coincides with the April 2016 eruption. Conversely, the peak in velocity occurs three months earlier in changes recorded by auto-correlations. The lack of consistency between these datasets could be related to the greater stability of the cross-components, with short-term changes typically only fluctuating between $\pm 0.05 \%$. Conversely, short-term fluctuations of $\pm 0.1 \%$ are common in velocities recorded by auto-correlations (Fig. 4.7a).

Velocity changes following the April-2016 eruption are significantly different from any other period. At WIZ station, velocities recorded by auto-correlations and cross-components fall by approximately $0.15 \%$ by the end of 2016, recording their lowest values since 2008-2009 (Fig. 4.7a and 4.7c). The most rapid period of this decline occurs 1-2 weeks before the September 2016 eruption, with a large velocity decrease recorded by auto-correlations and cross-components at both WIZ and WSRZ stations (Fig. 4.7a and 4.7c). At WIZ, the magnitude of this decrease is over $0.05 \%$, while at WSRZ a decrease of over $0.1 \%$ is observed. The timing of this change is similar to the velocity decrease recorded by the station-pair dataset (Fig. 3.18), suggesting this is a significant event relative to the 10-year study period.

Strong changes are also observed in measurements of the correlation coefficient of 0.1-1.0 Hz noise over the 10-year period (Fig. 4.7). The most significant of these changes, relative to background behaviour, are observed in the cross-component results (Fig. 4.7d); we therefore focus on these changes. Prior to 2012, the correlation coefficient fell below 0.85 only once in the previous five years. Since the onset of eruptive activity in 2012 however, the correlation coefficient has fallen below this value multiple times at both WIZ and WSRZ stations. The largest decrease occurs in early-2013, coinciding with the period of minor eruptive activity at the volcano. A further large decrease occurs in the 4-5 months preceding the April 2016 eruption, measured at both WIZ and WSRZ stations, and also at WIZ station in the 1-2 
(a)

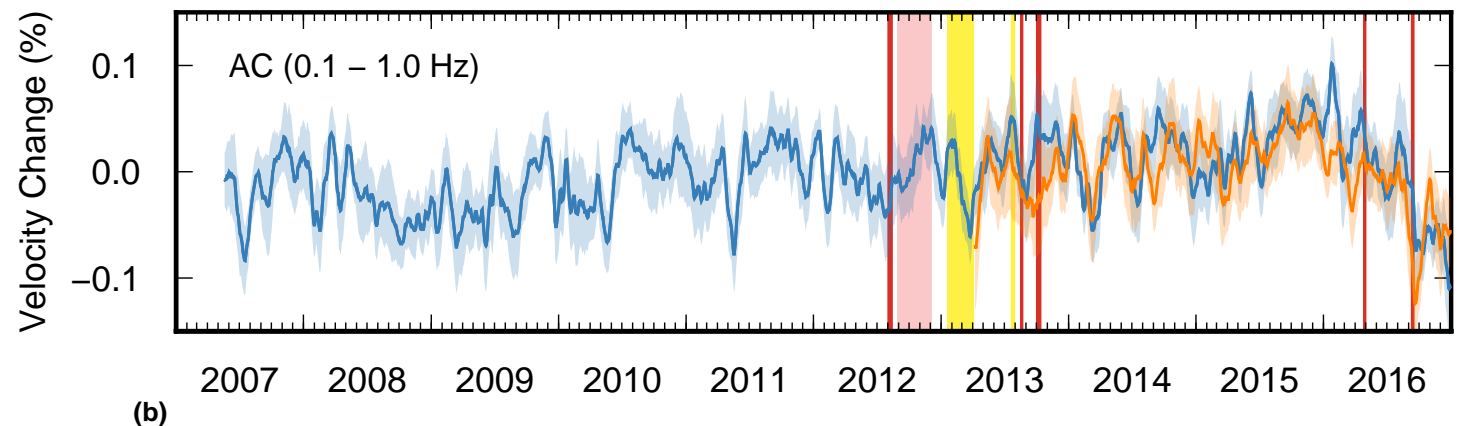

(b)

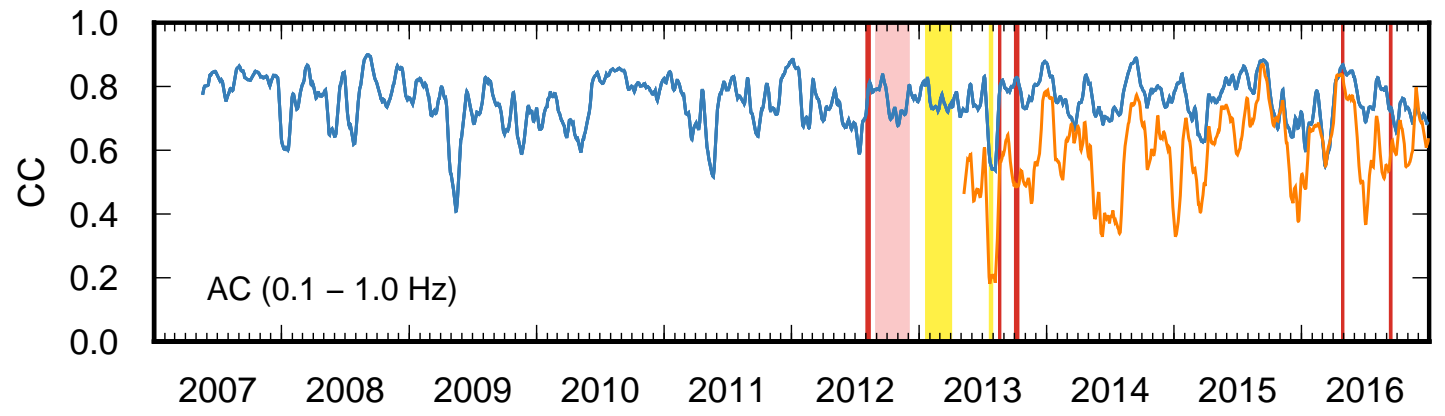

(c)

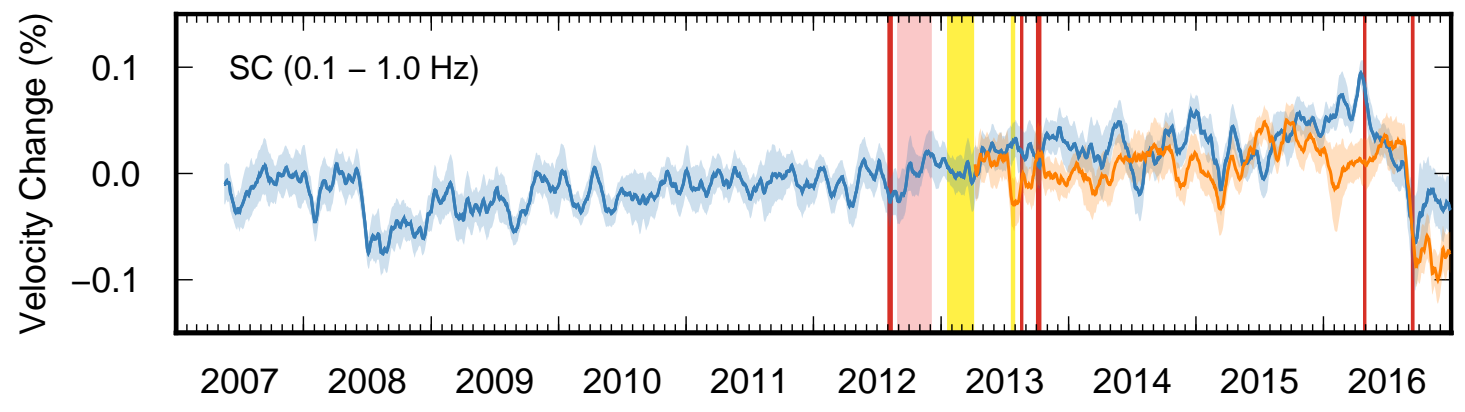

(d)

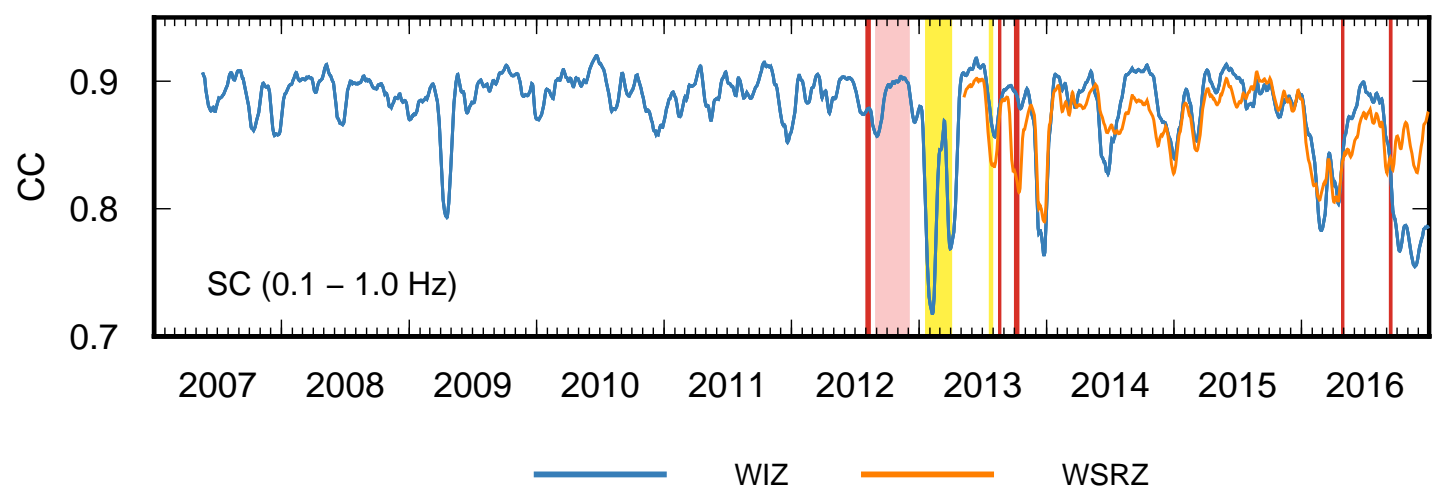

Eruption $\square$ Dome Growth $\square$ Minor Eruptive Activity

Figure 4.7: Averaged velocity changes and correlation coefficient measurements for 0.1-1.0 Hz auto-correlations (AC) and cross-component correlations (SC) at WIZ and WSRZ stations, smoothed with a 30-day centered moving-window. (a) Velocity changes for AC (b) CC measurements for AC. (c) Velocity changes for SC. (d) CC measurements for SC. 
weeks before and following the September 2016 eruption. This has not recovered by the end of 2016, possibly reflecting locational differences in the style of deformation. Similarly, the correlation coefficient recorded at WSRZ station does not appear to return to pre-April 2016 levels.

Closer inspection of the long-term velocity trend recorded by $0.1-1.0 \mathrm{~Hz}$ autocorrelations and cross-components at WIZ station reveals that the velocity does not increase continuously from 2008-2016 (Fig. 4.8). Instead, increases occur in at least three stages separated by periods of minimal long-term change. This is best revealed by the cross-components, due to their improved stability. The first stage of increase occurs immediately following the velocity decrease in 2008, with the velocity increasing until early-2011. Between 2011 and mid-2012, velocities remain relatively static, though begin to increase again at the start of activity in 2012. This continues at an approximately constant rate through to the October 2013 eruptions, before plateauing late-2013. Long-term velocities remain relatively steady from early-2014 to mid-2015, with a shorter-term velocity increase detected from late-2014 to early-2015. Interestingly, long-term velocity changes recorded by WSRZ station - having become active in 2013 - appear to have reversed polarity relative to velocity changes recorded by $0.1-1.0 \mathrm{~Hz}$ cross-components at WIZ station during this period. This is also the case for the final stage of velocity increase at

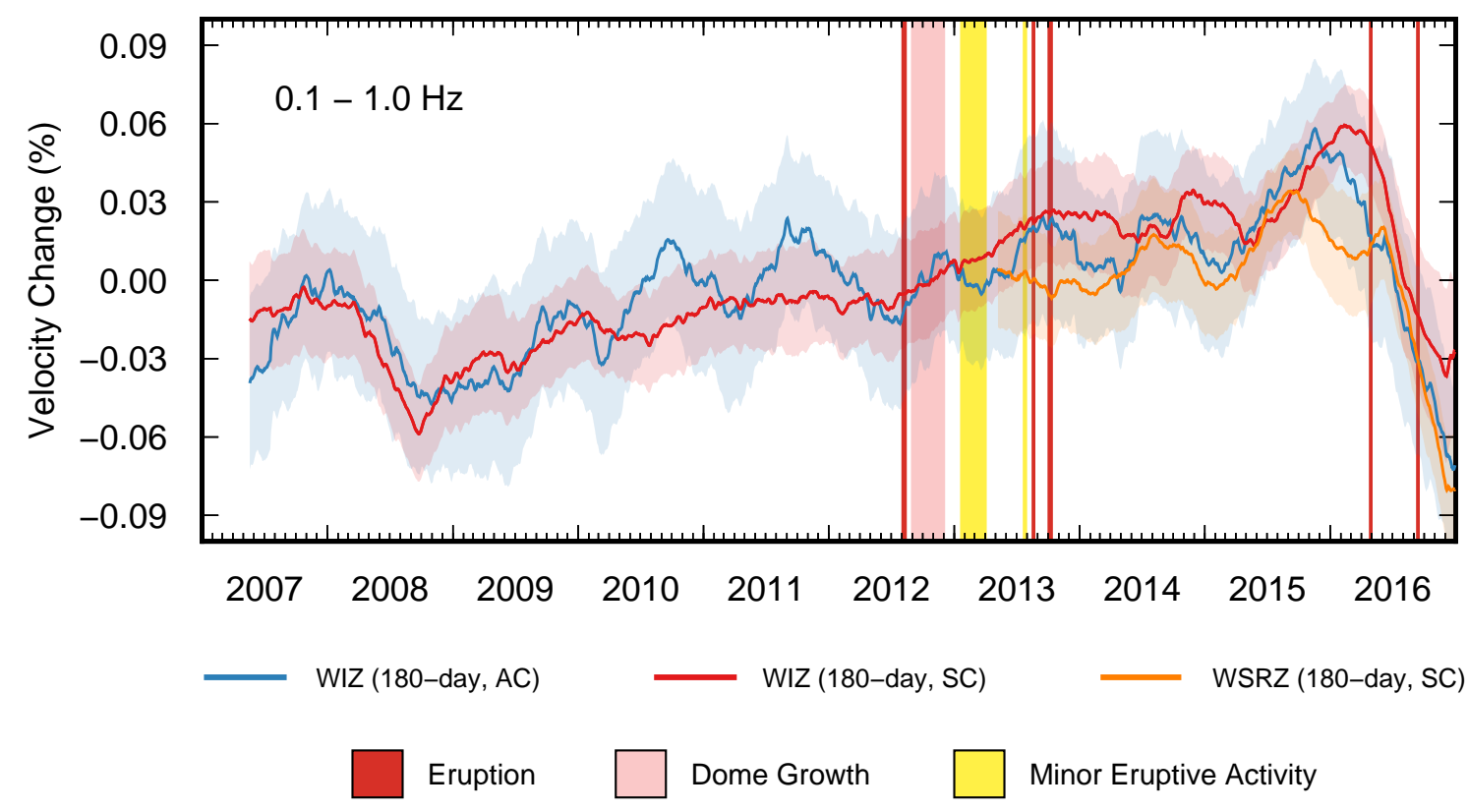

Figure 4.8: Long-term velocity changes (smoothed by 180-days) recorded by $0.1-1.0$ $\mathrm{Hz}$ auto-correlations at WIZ station and $0.1-1.0 \mathrm{~Hz}$ cross-components at both WIZ and WSRZ stations. 
WIZ station from mid-2015 through to the April 2016 eruption. The rate of increase during this period is larger than any other stage of increase described.

Results from 1.0-2.0 Hz auto-correlations and cross-components are shown in Figure 4.9. Similar features exist between $1.0-2.0 \mathrm{~Hz}$ and $0.1-1.0 \mathrm{~Hz}$ velocity changes, especially in the auto-correlations (Fig. 4.9a). A velocity decrease is still clearly observed in 2008, occurring just before a similar long-term velocity increase leading up to 2012. The magnitude of the velocity decrease is approximately double that of previous measurements, though is comparable relative to the size of background fluctuations. Beyond 2012 however, the nature of velocity changes recorded by 1.0 $2.0 \mathrm{~Hz}$ auto-correlations and cross-components differ substantially from $0.1-1.0 \mathrm{~Hz}$ changes. Velocities recorded by the auto-correlations at WIZ are reduced throughout 2012-2013 eruptive activity relative to pre-eruptive levels (Fig. 4.9a). Conversely, velocities recorded by the cross-components do not show significant changes during this period relative to levels pre-2012 (Fig. 4.9c). A clear change is however observed following the October 2013 eruption sequence, with a permanent velocity increase of approximately $0.05 \%$ recorded by both WIZ and WSRZ stations (Fig. 4.9c). Activity post-2013 consists of large amplitude changes recorded by autocorrelations from 2014-2016 (Fig. 4.9a), including a rapid increase of over $0.2 \%$ following the April 2016 eruption. This increase remains relatively stable for a few months before returning to pre-April 2016 levels in September. During the same period, velocity changes recorded by WSRZ auto-correlations undergo a significant decline, accumulating a total change of $0.4 \%$ from April-2016 to the end of the year (Fig. 4.9a). Interestingly, velocity changes recorded by WSRZ cross-components remain relatively unaffected during this period (Fig. 4.9c).

Large changes are also observed in 1.0-2.0 Hz correlation coefficients, especially during eruptive activity in 2012 - 2013 (Fig. 4.9b and 4.9d). For both auto-correlations and cross-components, the correlation coefficient is decreases from early-2012, reaching a minimum late-2012 (Fig. 4.9b and 4.9d). This has partially recovered by mid2013, following minor eruptive activity, though remains relatively unstable compared to pre-2012 levels. Further decreases occur prior to eruptive activity in 2016, with both auto-correlations and cross-components recording a correlation coefficient below background levels in late-2015 to early-2016. Behaviour following the April-2016 is variable between the components, with a permanent decrease recorded by WSRZ auto-correlations that has not recovered by the end of the year. No similar change is recorded by the cross-components, though a permanent decrease is recorded at WIZ following activity in September 2016 that is not observed in the auto-correlations. In relation to the changes in 2012-2013, we note that the correlation coefficient falls to 
(a)

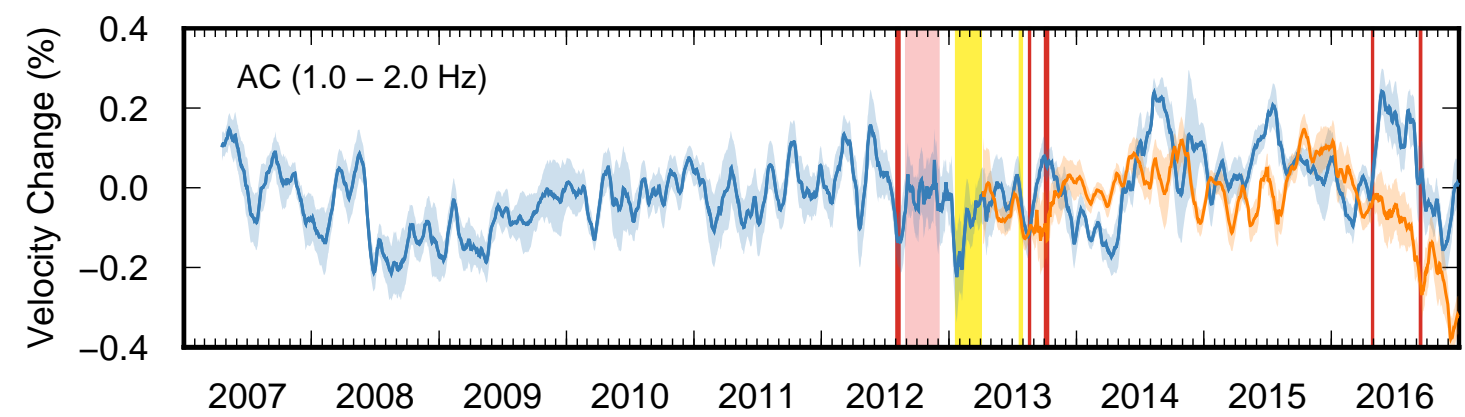

(b)

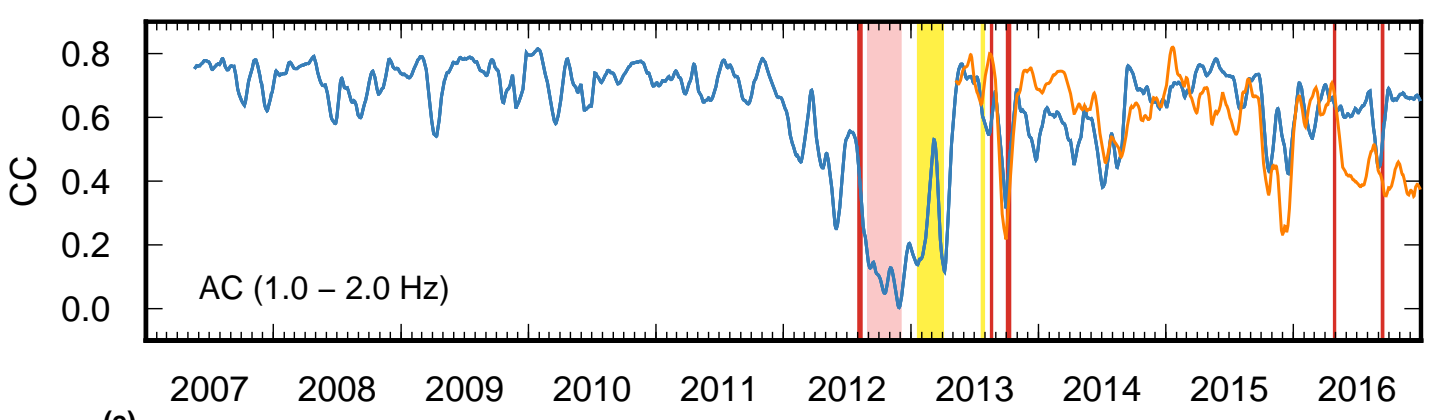

(c)
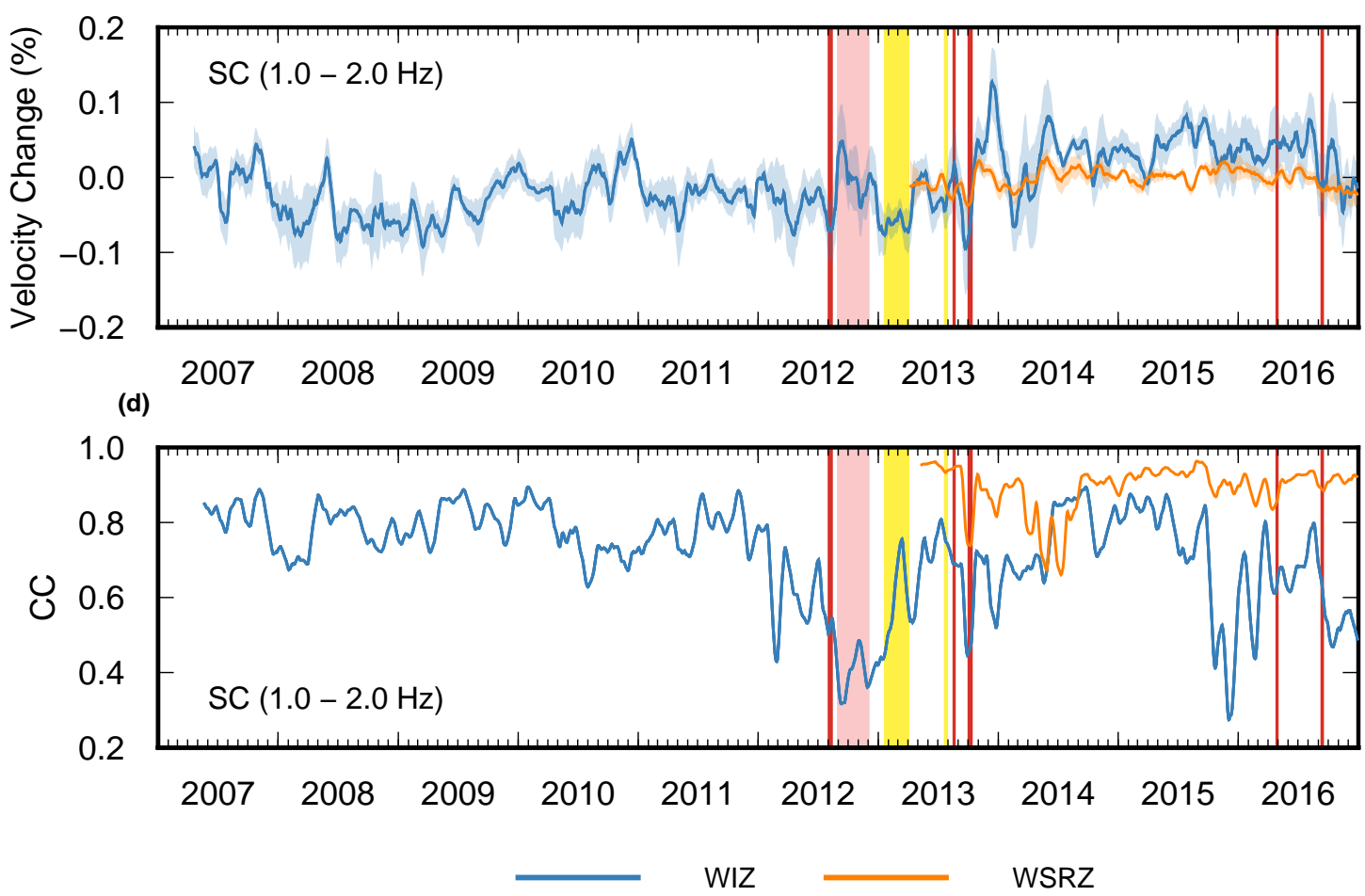

$\begin{array}{ll}\text { Eruption } & \text { Dome Growth } \square \text { Minor Eruptive Activity }\end{array}$

Figure 4.9: Averaged velocity changes and correlation coefficient measurements for 1.0-2.0 Hz auto-correlations (AC) and cross-component correlations (SC) at WIZ and WSRZ stations, smoothed with a 30-day centered moving-window. (a) Velocity changes for AC (b) CC measurements for AC. (c) Velocity changes for SC. (d) CC measurements for SC. 
values near zero, which corresponds to no correlation between current and reference stacks. This hints at contamination of the cross-correlation functions rather than a structural change, discussed further in Section 5.3.1).

Similarities between 1.0-2.0 Hz auto-correlations and $0.1-1.0 \mathrm{~Hz}$ cross-components suggest the sampling of similar source processes. This is demonstrated in Figure 4.10a, where velocity changes recorded by WIZ $0.1-1.0 \mathrm{~Hz}$ cross-components are compared with 1.0-2.0 Hz auto-correlations. Both time series are normalised between -1 and 1 in order to aid visual comparison. Strong agreement between the two time series is observed prior to eruptive activity in 2012, with both showing a clear velocity decrease mid-2008 in addition to the long-term increase in the following years. The behaviour changes however following the August 2012 eruption, with stronger deviations between the two time series. Interestingly, these differences appear to be inversely proportional to each other at times, potentially indicating a different response to the same source. Noting that velocity changes recorded by
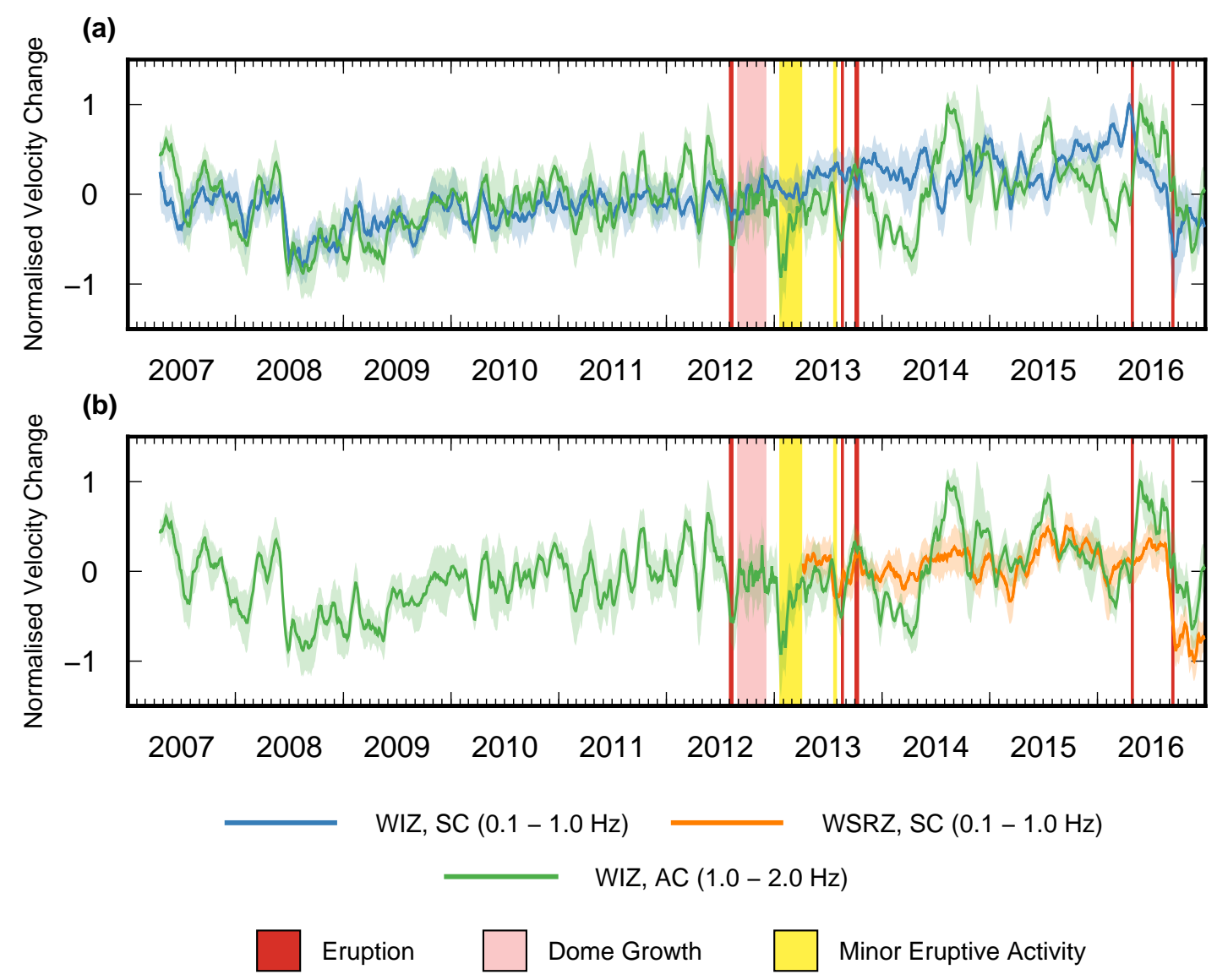

Figure 4.10: Comparison of velocity changes recorded by $1.0-2.0 \mathrm{~Hz}$ auto-correlations at WIZ with: (a) $0.1-1.0 \mathrm{~Hz}$ cross-components at WIZ, (b) $0.1-1.0 \mathrm{~Hz}$ crosscomponents at WSRZ. Velocity changes are normalised between -1 and 1 to improve visual comparison. 
0.1-1.0 Hz cross-components at WSRZ exhibited a similar inverse relationship with WIZ station (Fig. 4.8), we compare this dataset with 1.0-2.0 Hz auto-correlations in Figure 4.10b. A good fit is observed from late-2014 to mid-2016, potentially supporting a common source process.

\subsubsection{Comparison with Onshore Velocity Changes}

Onshore seismic stations can provide valuable information about the nature of nonvolcanic processes that may be influencing velocities recorded at White Island. In this section, we examine measurements recorded by onshore stations, averaging velocity changes processed for HAZ, MWZ and PUZ stations to construct an onshore dataset. This aims to average out individual differences based on station locations, instead capturing changes on a regional scale. We exclude OPRZ station from this average due to the presence of high amplitude, long-term changes that were not representative of other onshore stations (Appendix B.3). We also exclude URZ station, as only vertical component data were obtained.

Differences are observed in onshore velocity changes relative to those observed at White Island stations (Fig. 4.11). We see no evidence of a similar long-term in-

(a) $\mathrm{AC}(0.1-1.0 \mathrm{~Hz})$

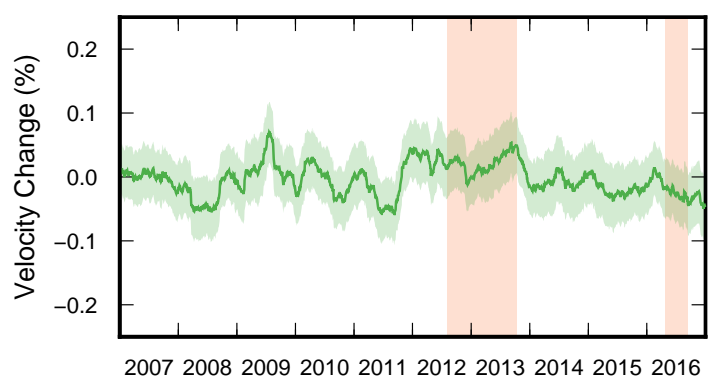

(c) $\mathrm{AC}(1.0-2.0 \mathrm{~Hz})$

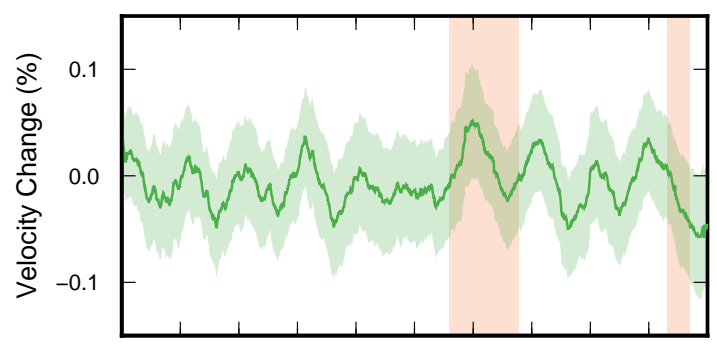

2007200820092010201120122013201420152016 (b) SC $(0.1-1.0 \mathrm{~Hz})$

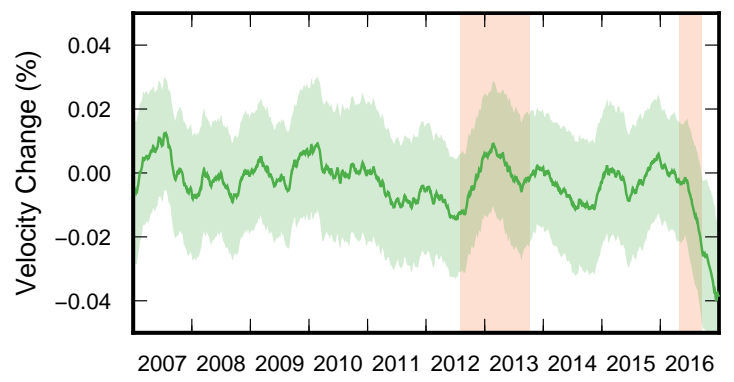

(d) SC $(1.0-2.0 \mathrm{~Hz})$

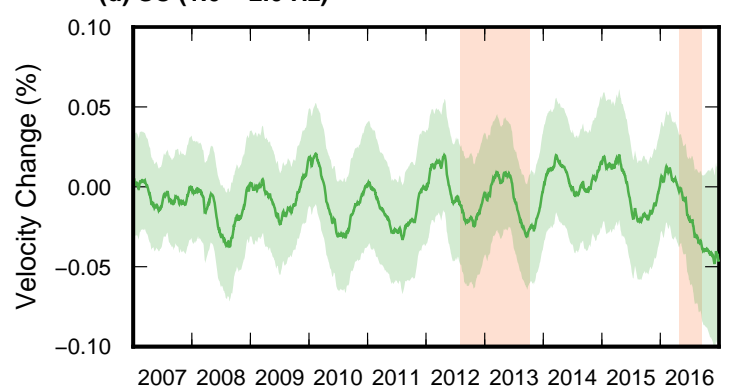

Figure 4.11: Velocity changes are shown for the onshore dataset, representing the average of single-stations HAZ, MWZ and PUZ. Both auto-correlations (AC) and cross-components (SC) are shown, smoothed by 180-days. Periods of eruptive activity are shaded in light red. (a) AC, 0.1-1.0 Hz. (b) SC, 0.1-1.0 Hz. (c) AC, 1.0-2.0 Hz, (d) SC, 1.0-2.0 Hz. 
(a)

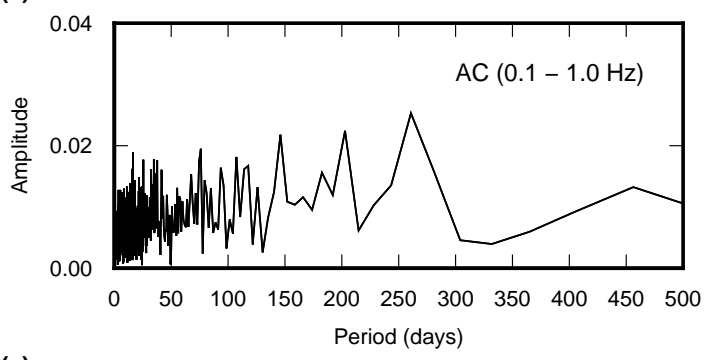

(c)

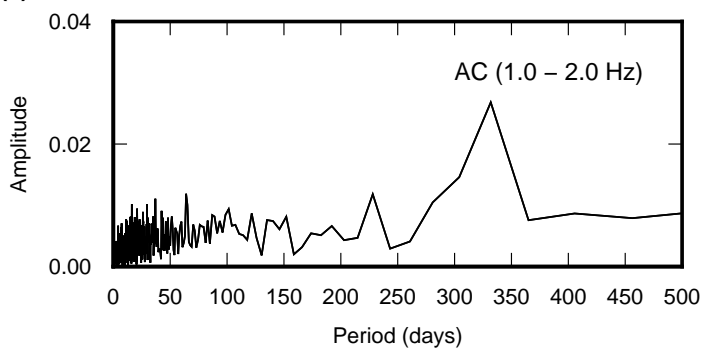

(b)

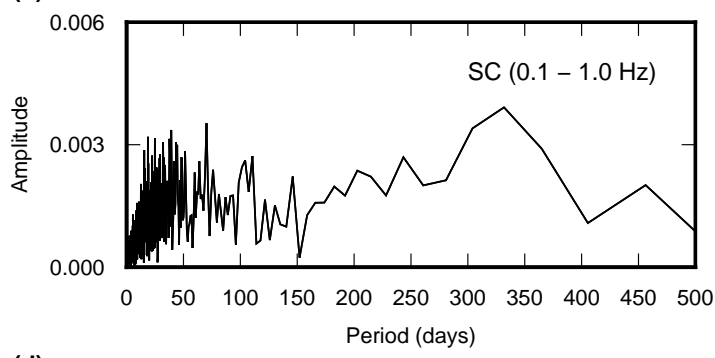

(d)

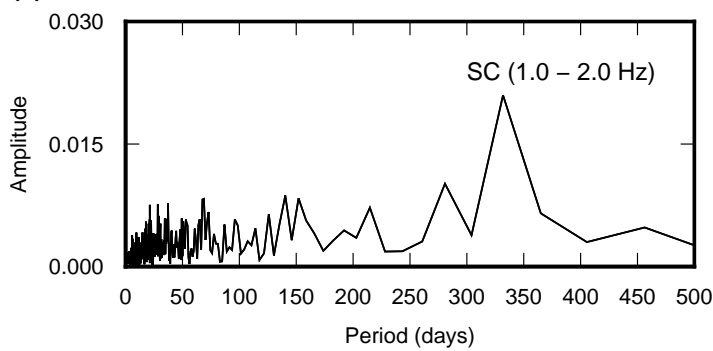

Figure 4.12: Fast Fourier Transform of velocity changes measured by onshore stations (average of HAZ, MWZ and PUZ stations). (a) Auto-correlations, 0.1-1.0 Hz. (b) Cross-components, 0.1-1.0 Hz. (c) Auto-correlations, 1.0-2.0 Hz. (d) Crosscomponents, 1.0-2.0 Hz.

crease, with velocities instead remaining relatively stable over the 10-year period. Approximately annual changes are clearly observed, especially in 1.0-2.0 Hz velocity changes (Fig. 4.11c and 4.11d). We once again perform a Fast-Fourier transform to better examine this periodicity, with resultant amplitude spectrums shown in Figure 4.12. Dominant peaks approximately corresponding to an annual period are clearly observed in 1.0-2.0 Hz auto-correlations and cross-components (Fig. 4.12c and 4.12d). A similar peak is also observed in $0.1-1.0 \mathrm{~Hz}$ cross-components, though is not as dominant relative to other periodicities in this dataset (Fig. 4.12b). Conversely, no annual periodicity is evident from the amplitude spectrum of $0.1-1.0 \mathrm{~Hz}$ auto-correlations (Fig. 4.12a).

The identification of annual periodicity in velocity changes onshore could be useful in determining whether changes at White Island are non-volcanic in their origin. This is perhaps best reflected in velocity changes recorded by $0.1-1.0 \mathrm{~Hz}$ cross-components, with strong similarities observed between velocities recorded onshore and velocities recorded at WIZ station (Fig. 4.13). This suggests that the same source process may be responsible for these similarities. Clearly missing however is the velocity decrease in 2008 and the subsequent velocity increase over the years following. This suggests that sources of these changes are either unique to White Island or, at the very least, not occurring regionally. Interestingly though, a significant velocity decrease is observed in the onshore dataset in 2016 around the time of volcanic activity in 
(a)
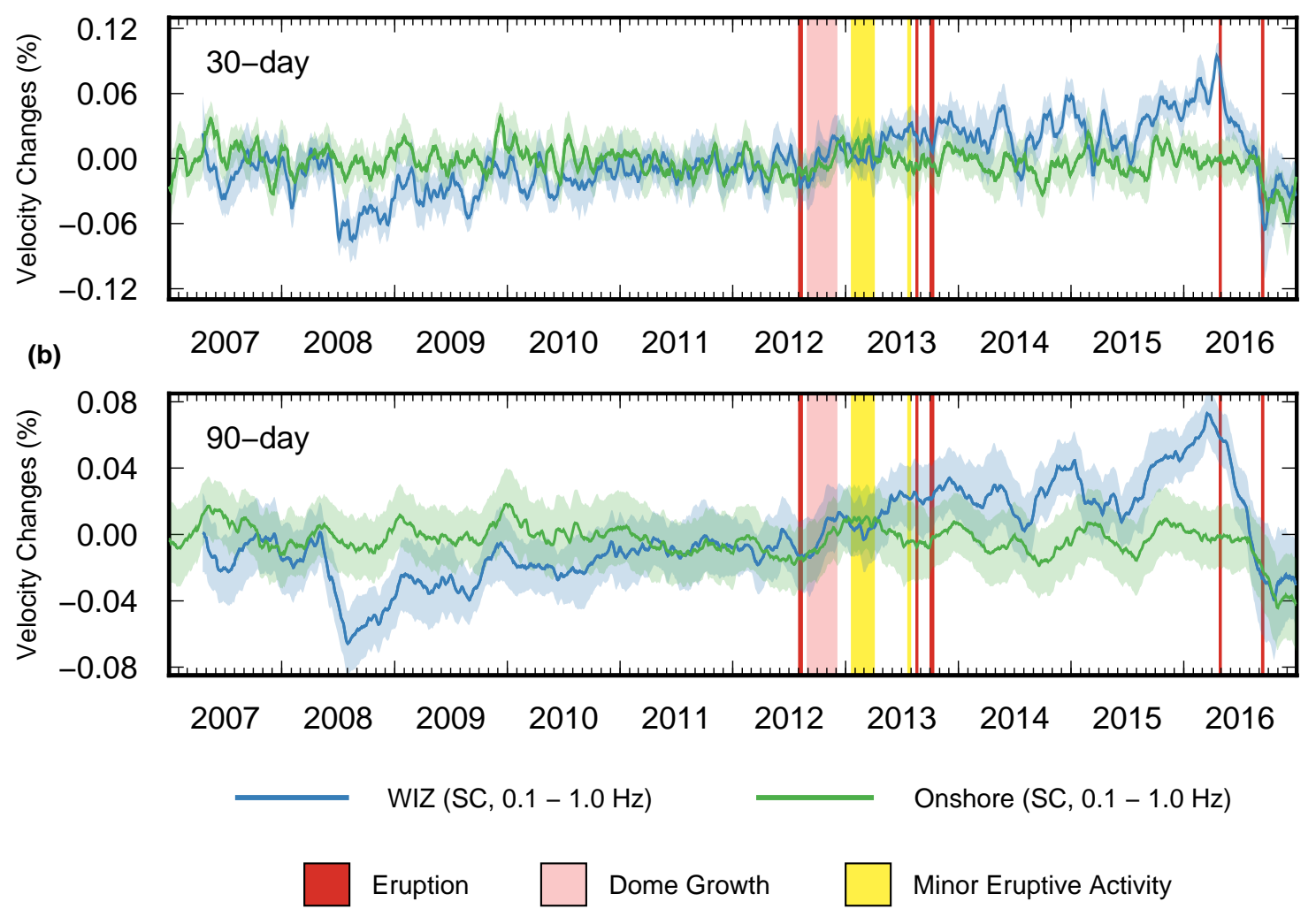

Figure 4.13: Comparison between velocity changes recorded by $0.1-1.0 \mathrm{~Hz}$ crosscomponents at WIZ station and recorded by onshore stations (average of HAZ, MWZ, PUZ). (a) Velocities smoothed by 30-days. (b) Velocities smoothed by 90-days.

September 2016. This occurs at the same time as velocity decreases recorded by White Island stations (e.g. Fig. 4.13a) and also changes recorded by the stationpair dataset in Chapter 3 (Fig. 3.18) Therefore it is likely this change reflects a regional velocity response to a non-volcanic source, with it unlikely a volcanic source - associated with the September 2016 eruption - would be detected so far from White Island.

\subsubsection{Comparison with Tectonic Earthquakes}

The onshore emergence of a significant velocity decrease in September 2016 (Fig. 4.13) suggests a regional change is responsible. We therefore broaden our search for the source process to include natural phenomena capable of producing widespread changes. One notable event that occurred during this time was the Te Araroa earthquake sequence in northeast New Zealand (Fig. 4.14a). This began with a $M_{W} 5.7$ foreshock on the 31 August (UTC) and was followed less than 24 hours later by a $M_{W} 7.1$ mainshock at $19 \mathrm{~km}$ depth (Warren-Smith et al., 2018). The timing of this event is consistent with changes in seismic velocities both onshore and at White Island (Fig. 4.14b, c and d) and therefore represents a likely source, despite its 
epicenter more than 200 kilometers from the volcano.

Following the detection of an induced co-seismic velocity change associated with the $M_{W}$ 7.1 Te Araroa earthquake, we extend our search for tectonic earthquake induced changes to nearby earthquakes over magnitude five between 2007 - 2017. Two of these events are shown in Figure 4.14e: (1) a magnitude $M_{W} 6.7$ earthquake at 33 $\mathrm{km}$ depth (GeoNet located) and (2) a magnitude $M_{W} 5.2$ earthquake at $5 \mathrm{~km}$ depth (GeoNet located). The magnitude $M_{W} 6.7$ is an obvious choice to investigate further, given the comparable size to the Te Araroa earthquake. However, no obvious changes associated with this earthquake are detected by onshore stations (Fig. 4.14g) or by WIZ station (Fig. 4.14f). Conversely, the magnitude $M_{W} 5.2$ earthquake, while not being as large, is located within $10 \mathrm{~km}$ of the volcano and at a shallow depth (Fig. 4.14e). The timing of this event coincides perfectly with the strong velocity decrease recorded by WIZ station in 2008 (Fig. 4.14f). Thus, we are confident that the magnitude $M_{W} 5.2$ earthquake is responsible for this velocity decrease. 

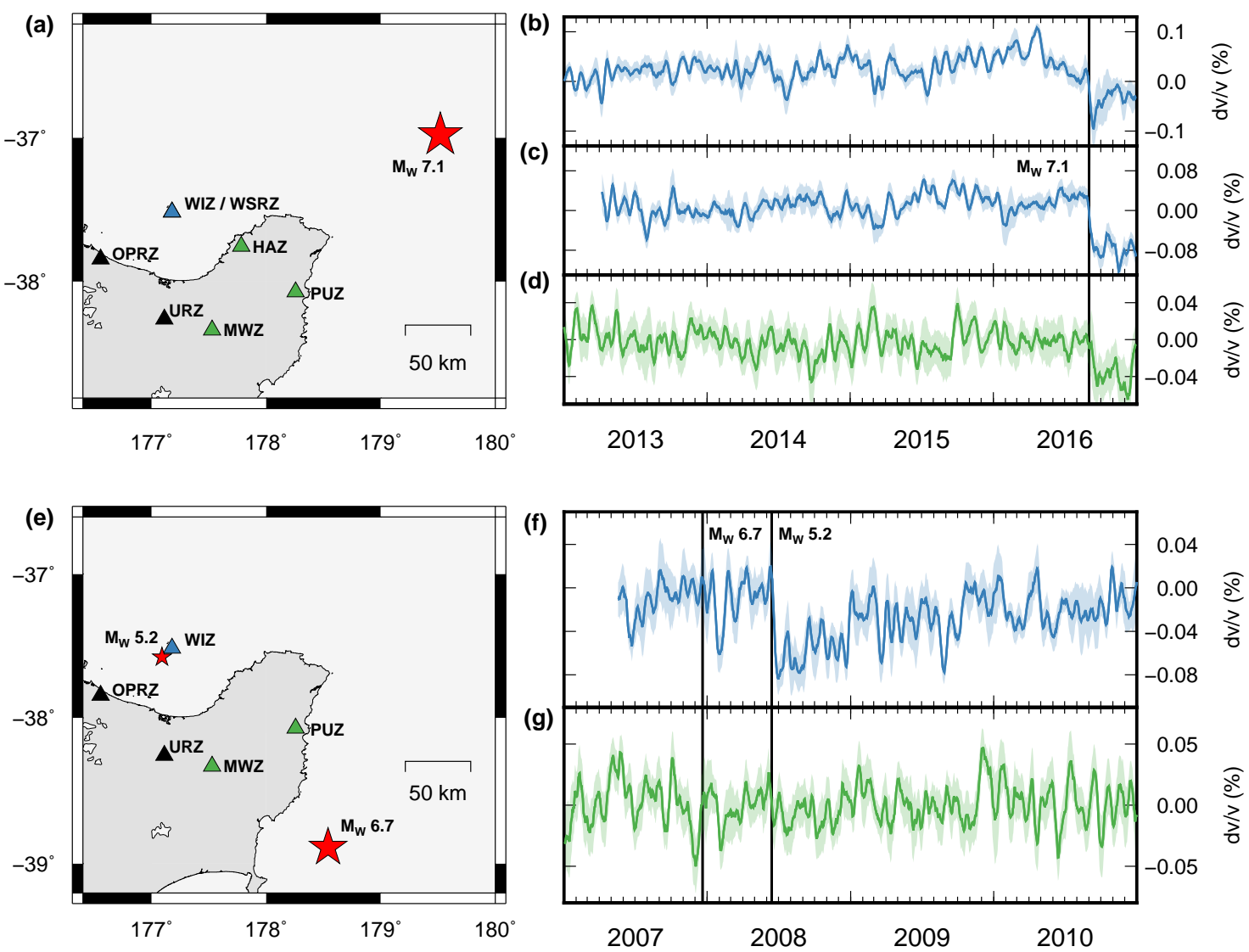

Figure 4.14: Single-station velocity changes recorded by $0.1-1.0 \mathrm{~Hz}$ cross-components shown at times of nearby large tectonic earthquakes. Time series are colour coded with corresponding stations shown in map view. Velocities are smoothed by 14-days, reduced from 30-days to highlight co-seismic change. (a) Location of $M_{W}$ Te Araroa earthquake in 2016. (b) Velocity changes recorded at WIZ station. (c) Velocity changes recorded at WIZ station. (SC). (d) Velocity changes recorded by onshore stations (average of HAZ, MWZ and PUZ stations). (e) Location of $M_{W} 6.7$ Gisborne earthquake and $M_{W} 5.2$ earthquake in 2008. Stations not active at the time are not shown in map view. (f) Velocity changes recorded at WIZ station. (g) Velocity changes recorded by onshore stations (average of HAZ, MWZ and PUZ stations). Note that HAZ was not running at the time of the earthquake. 


\section{Chapter 5}

\section{Discussion}

The discussion is split into three parts. The first investigates the depth sensitivity of the results and considers whether the station-pair dataset is sampling changes within the vicinity of the volcano (Section 5.1). We then discuss non-volcanic background influences that could be present at the volcano (Section 5.2). This sets a platform for making informed judgements about whether velocity changes at the volcano represent volcanic sources (Section 5.3). We conclude by discussing the potential and limitations of real-time monitoring using ambient noise (Section 5.3.3).

\subsection{Sampling Depth and Location}

This section looks to establish the expected depth sensitivities of the different datasets used in this thesis based on the frequencies of surface waves involved (Section 5.1.1). Additionally, we address the question of whether the station-pair dataset is effectively sampling changes at the volcano, given large separation distances between onshore and offshore seismic stations (Section 5.1.2).

\subsubsection{Depth Sensitivity Kernels}

Depth sensitivity kernels are used to investigate how sensitive surface wave velocities are to changes at depth. For ambient noise, this aids in determining the depth of observed changes. The velocity of surface waves can be considered as directly proportional to the shear wave velocity (Hobiger et al., 2012). Depth sensitivity kernels are therefore obtained by taking the derivative of fundamental mode Rayleigh and Love wave group velocities with respect to shear wave velocities, computed for different periods based on codes written by Herrmann (2013). Group velocities are calculated using the velocity model of Eberhart-Phillips et al. (2010), detailed in Appendix A.5.

Depth sensitivity kernels are shown in Figure 5.1 for the minimum and maximum 

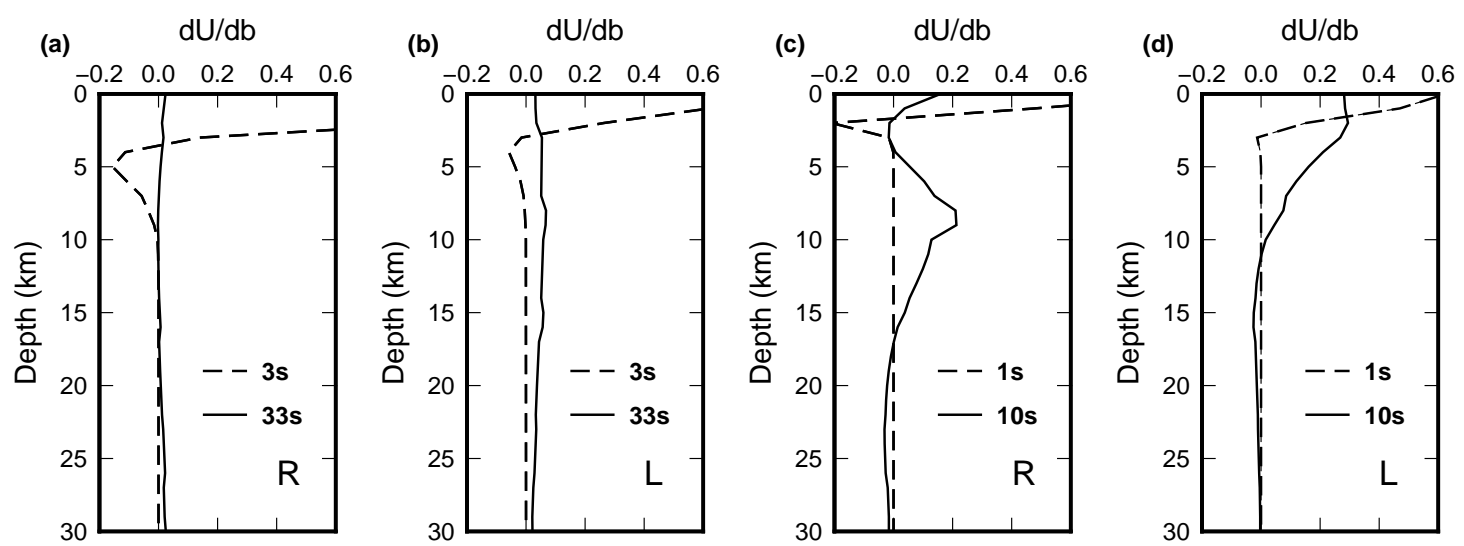

Figure 5.1: Depth sensitivity kernels computed for fundamental mode Rayleigh (R) and Love ( $\mathrm{L}$ ) waves at periods corresponding to the minimum and maximum periods of the datasets used in this thesis. (a) Rayleigh wave sensitivity kernels for periods 3 and 33 seconds (station-pair dataset). (b) Love wave sensitivity kernels for periods 3 and 33 seconds (station-pair dataset). (c) Rayleigh wave sensitivity kernels for periods 1 and 10 seconds (0.1-1.0 Hz single-station), (d) Love wave sensitivity kernels for periods 1 and 10 seconds $(0.1-1.0 \mathrm{~Hz}$ single-station).

periods of the different datasets in this thesis. Figures 5.1a and 5.1b represent kernels for noise used in the station-pair dataset (3-33 seconds period). Figures 5.1c and 5.1d represent kernels for noise used by the lower bound of the single-station dataset (1-10 seconds period). The station-pair dataset is shown be sensitive to velocity changes across a wide range of depths, with the shortest periods sensitive to the upper $5 \mathrm{~km}$ and the longest periods the entire crust. Conversely, the singlestation dataset is sensitive to the middle-crust at longer periods and the upper 2-3 $\mathrm{km}$ at shorter periods. The upper bound of this dataset (0.5-1 second period) is not shown, and can be assumed to be sensitive to the uppermost crust.

\subsubsection{Sampling Location}

One important outstanding question is whether the station-pair dataset is sampling velocity changes in the vicinity the volcano. We seek to test this assumption before investing further time into understanding changes recorded by this dataset. Fortunately, we have the advantage of comparing velocity changes recorded by stationpairs to single-station measurements on the volcano, assuming that these are sensitive to local changes. This is supported through the detection of a co-seismic velocity change following a nearby $M_{W} 5.2$ earthquake (Fig. 4.14f). Single-station results therefore serve as a platform for classifying whether velocites recorded by the station-pair dataset can be considered a local or regional change.

Earthquake induced velocity changes are perhaps the strongest line of evidence for 
isolating the locality of velocity perturbations, due to knowledge of both the timing and location of the source. Single-station results included two clear periods of coseismic velocity decrease associated with earthquake activity. The larger of the two earthquakes, a magnitude $M_{W} 7.1$ in 2016, was located over $200 \mathrm{~km}$ away from the volcano (Fig. 4.14a). Changes associated with this event are recorded throughout the region, both at the volcano and by stations onshore (Fig. 4.14). A seismic velocity decrease in the station-pair dataset coinciding with this event (Fig. 3.20) therefore does little to verify the sampling location. It does however, at the very least, show that crustal velocity changes are being correctly sampled by the stationpairs. The smaller earthquake, a magnitude $M_{W} 5.2$ in 2008, was located within 10 $\mathrm{km}$ of the volcano (Fig. 4.14e). This event provides a more valuable comparative tool, with vast regional velocity changes unlikely owing to its smaller size.

No obvious co-seismic changes are observed in the station-pair dataset at the time of the $M_{W} 5.2$ earthquake, with the only significant change a sharp velocity increase 3-4 months later (Fig. 3.20). A velocity low is identified in the long-term trend however (Fig. 3.20), with OPRZ station found to be a dominant influence (Fig. 3.23, demonstrated again in Figure 5.2). It might be expected that the station-pair OPRZ-WIZ would be the most likely to detect a change relating to this earthquake, given its epicenter between the two stations (Fig. 4.14e). However, although the long-term velocity low appears to perfectly coincide with this event (Fig. 5.2b), short-term velocities suggest that OPRZ-WIZ was introducing reduced velocities into the average at least 3-4 months beforehand (Fig. 5.2a). Thus, we do not believe there is sufficient evidence to suggest the station-pair dataset has sampled a velocity decrease associated with the $M_{W} 5.2$ earthquake.

One complication associated with comparing the two datasets is the minimal overlap of sampled frequencies. Velocity changes recorded by the station-pair dataset (0.03$0.33 \mathrm{~Hz})$ are at the lower end of frequencies sampled by single-station results (0.1$1.0 \mathrm{~Hz}$ ). This introduces an ambiguity associated with determining whether missing features from the station-pair results reflect a different sampling location or a depth dependency. For example, the lack of a long-term velocity increase in the station-pair dataset could be explained as a consequence of reduced sensitivity to the upper crust (Fig. 5.1). We choose to not explore velocity changes recorded by the station-pair dataset further without clear evidence to support a link to changes at the volcano. 
(a)

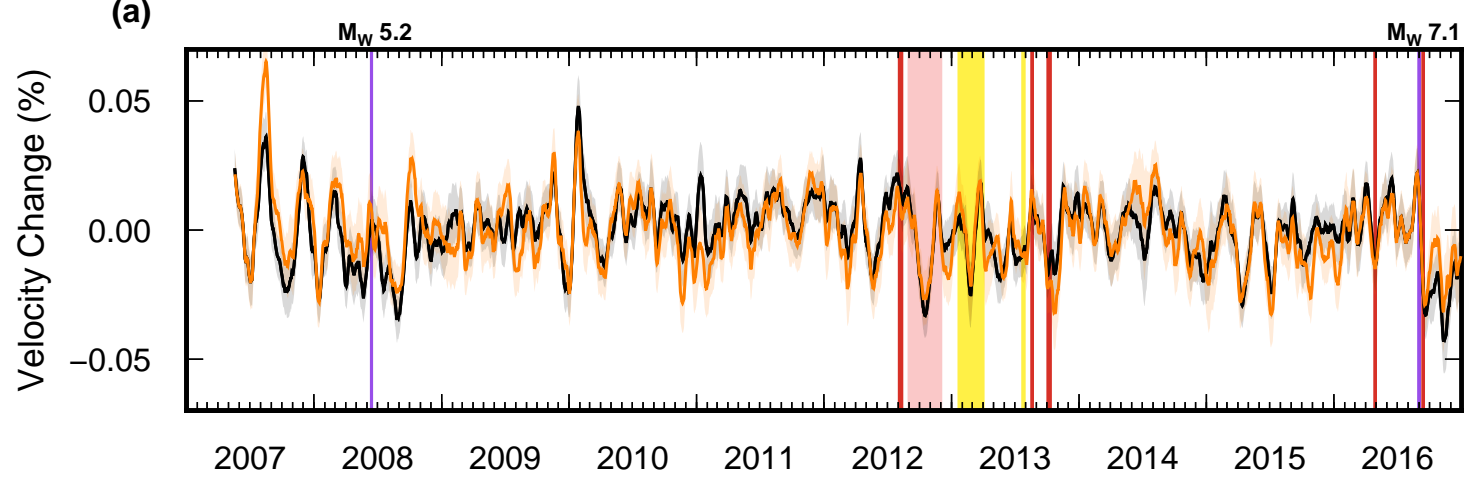

(b)

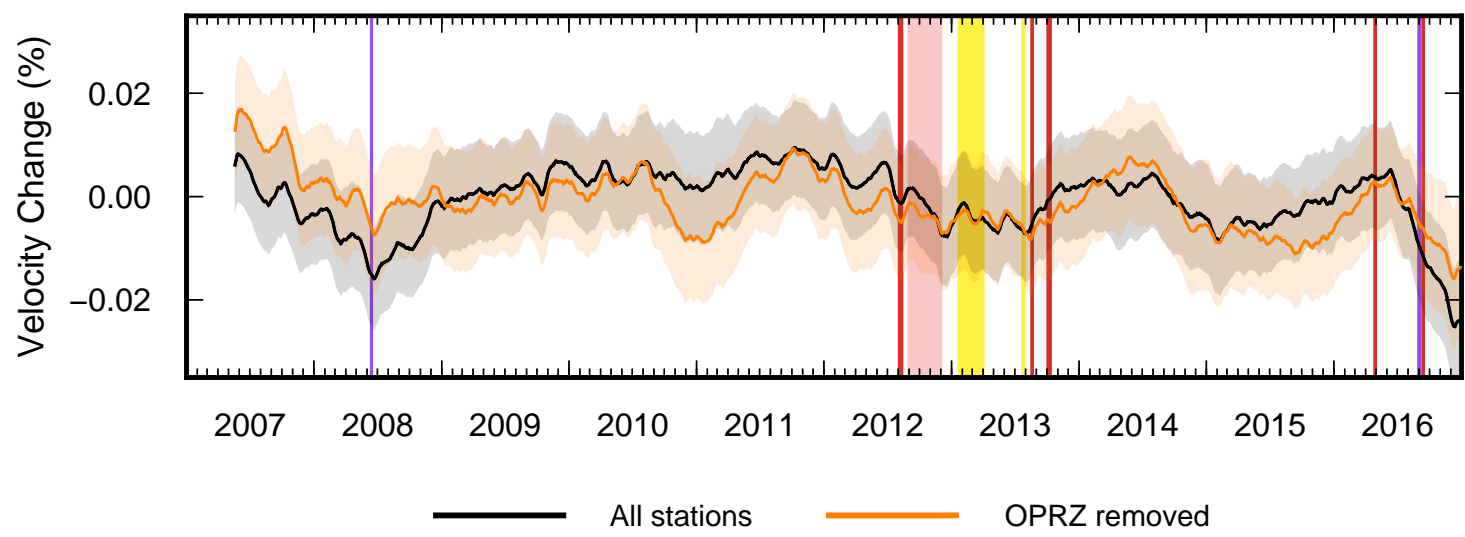

Eruption

Dome Growth

Minor Eruptive Activity

Earthquake

Figure 5.2: Influence of OPRZ station on WIZ averaged time series - from stationpair dataset - with earthquake activity highlighted. Black line is the average of all station-pairs, orange line is the average minus OPRZ-WIZ. (a) Smoothed by a 14-day window. (b) Smoothed by a 180-day window.

\section{$5.2 \quad$ Background Sources}

Many non-volcanic processes have been found to influence seismic velocities in the crust, producing changes with magnitudes similar to those produced by volcanic activity. Successful ambient noise monitoring should therefore consider the presence of background influences when making interpretations about observed velocity changes. We use this section to explore background sources of velocity changes at White Island, discussing possible meteorological influences and changes related to tectonic seismicity. 


\subsubsection{Environmental Factors}

Seasonal influences on seismic velocities are well recognised, with links to precipitation (e.g. Sens-Schönfelder and Wegler, 2006; Hillers et al., 2014), atmospheric pressure loading (e.g. Silver et al., 2007; Niu et al., 2008), and atmospheric temperature variations (e.g. Meier et al., 2010; Richter et al., 2014; Hillers et al., 2015). Recent studies have started to model synthetic velocity changes based on these background influences (e.g. Tsai, 2011; Wang et al., 2017). At Piton de la Fournaise (Rivet et al., 2014) and Merapi volcano (Budi-Santoso and Lesage, 2016), corrections have been made to velocity changes to account for observed correlations with rainfall. No volcano is the same however and, with different regions capable of producing varied crustal responses (e.g. Wang et al., 2017), seasonal changes observed elsewhere may not necessarily be observed at White Island.

Clear evidence of environmental influence on velocities is recorded by onshore single stations, with strong peaks in the amplitude spectrum at an approximate annual period (Fig. 4.12). We therefore use this dataset to investigate possible environmental influences. Unlike White Island stations, onshore stations are not influenced by volcanic processes and therefore contain valuable information about background changes that could be present at the volcano. Figure 5.3 shows the average yearly trends for onshore single-station velocity changes, smoothed using a 90-day window to bring out seasonal characteristics. Clear seasonal behaviour is observed in 1.0$2.0 \mathrm{~Hz}$ velocity changes, with peaks during New Zealand summer months (Fig. 5.3c and 5.3d). At lower frequencies, seasonal behaviour is less obvious, though is partly observed by $0.1-1.0 \mathrm{~Hz}$ cross-components with velocities peaking 2-3 months earlier (Fig. 5.3b). Seasonal behaviour is not apparent in $0.1-1.0 \mathrm{~Hz}$ auto-correlations, with no clear peak and significantly higher amplitude changes (Fig. 5.3a). Similar high amplitude changes at low frequencies were recorded by Hillers et al. (2015), who suggested the possibility of bias associated with temporal variability in the microseism wavefield — which is dominant at lower frequencies. Contamination of auto-correlations is a known issue, with no spectral whitening applied during preprocessing.

We investigate four meteorological datasets as possible sources of observed seasonality. These are rainfall, temperature and atmospheric pressure and wind speed changes. Wind speed changes do not produce a real physical change, but can introduce a bias through altering the properties of seismic noise at frequencies above approximately $1 \mathrm{~Hz}$ (Hillers et al., 2015). Temperature and wind speed measurements are recorded by a MetService owned weather station on White Island. Precipitation and atmospheric pressure measurements are obtained through a Whakatane 
(a)

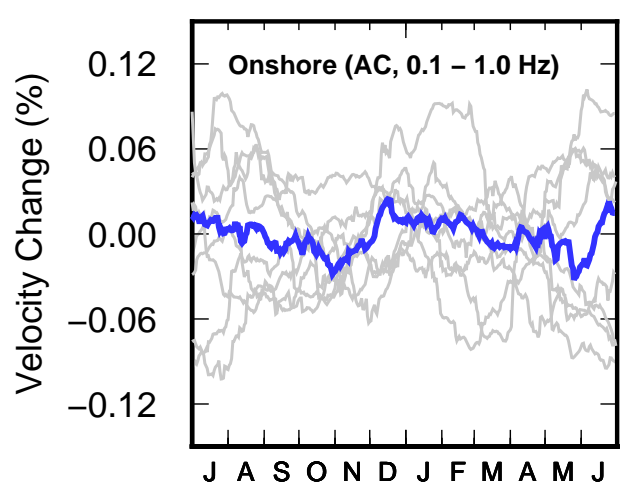

(c)

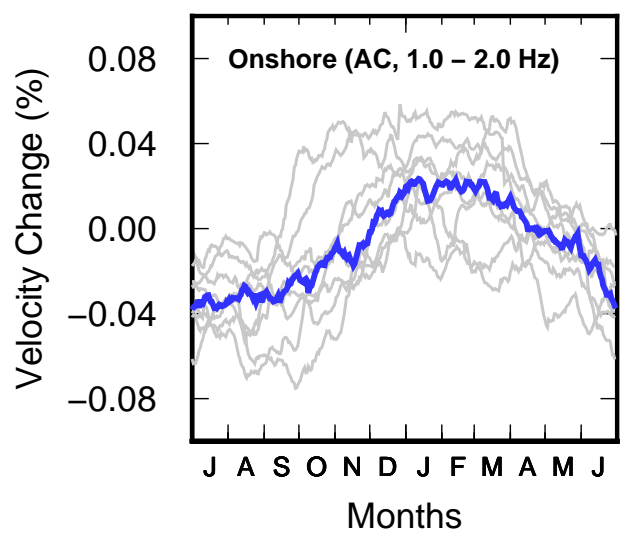

(b)

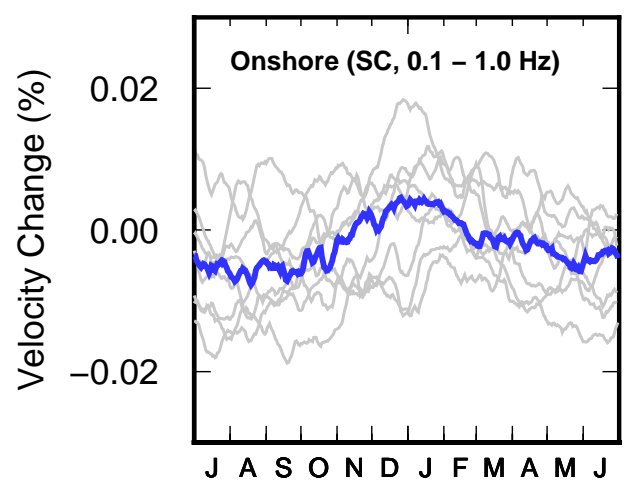

(d)

Months

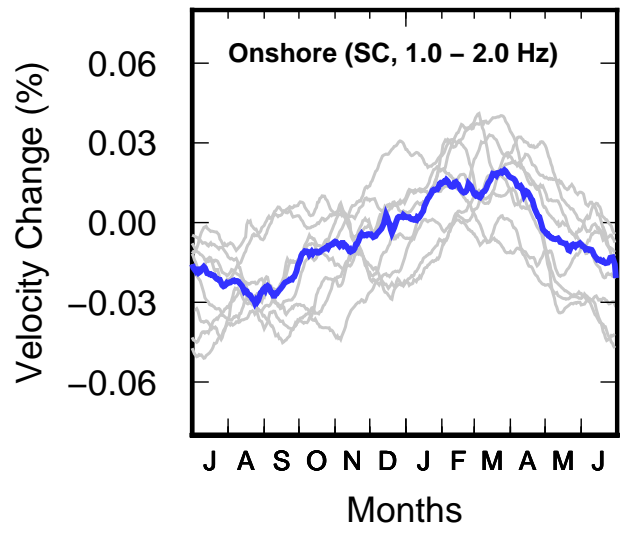

Figure 5.3: Yearly trend of velocity changes recorded by auto-correlations (AC) and cross-component correlations (SC) at onshore single stations (average of HAZ, MWZ and PUZ), smoothed with a 90-day moving window. Individual years of changes are plotted from July to July of the following year (grey lines) with the median calculated (blue lines). (a) AC, 0.1-1.0 Hz. (b) SC, 0.1-1.0 Hz (c) AC, 1.0-2.0 Hz. (d) SC, 1.0$2.0 \mathrm{~Hz}$.

station located $50 \mathrm{~km}$ from the island. The yearly trend of these datasets is shown in Figure 5.4. We acknowledge that some variability will exist between environmental conditions onshore and offshore that prevent a more complete analysis of seasonal behaviour. Such a target is outside the scope of this thesis. However, we expect conditions to be stable enough over the Bay of Plenty region to warrant a simplified investigation into the environmental sources of velocity change.

Meteorological processes are not independent of each other, potentially complicating any interpretation of the primary driver of velocity changes. In Figure 5.4, we see that the seasonal characteristics of atmospheric pressure changes and rainfall are unique relative to other meteorological datasets. Peaks in temperatures at White Island (Fig. 5.4c) however occur at the same time as the lowest wind speeds (Fig. 5.4d). Fortunately, we can distinguish between these two processes based on the the timing of the velocity response. Temperature-dependent changes occur through 
(a)

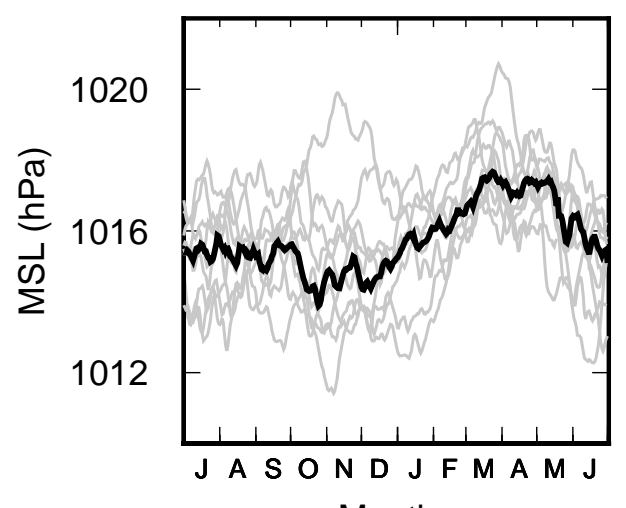

(c)

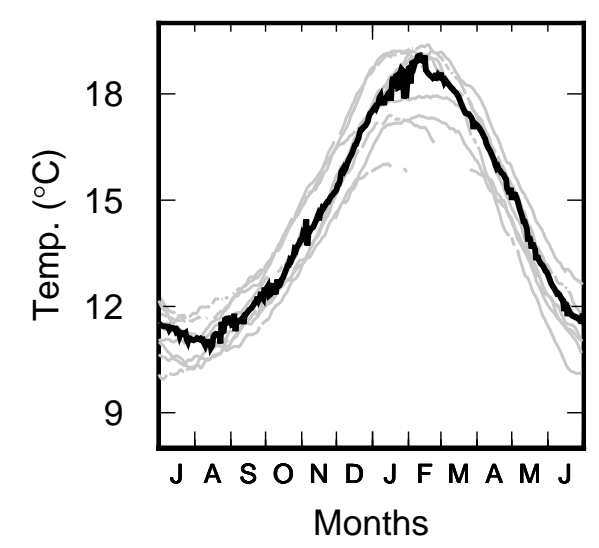

(b)

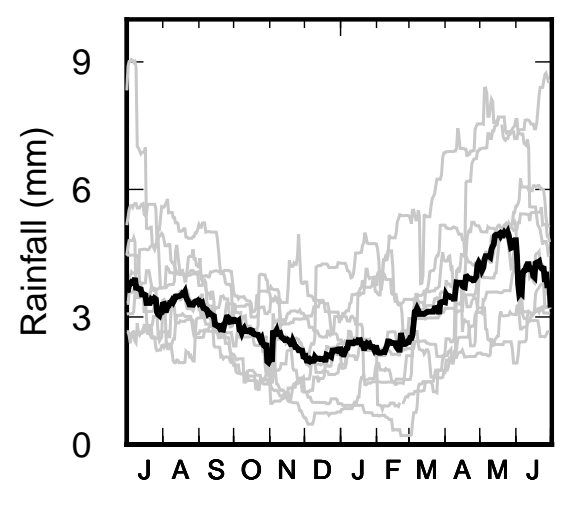

(d)

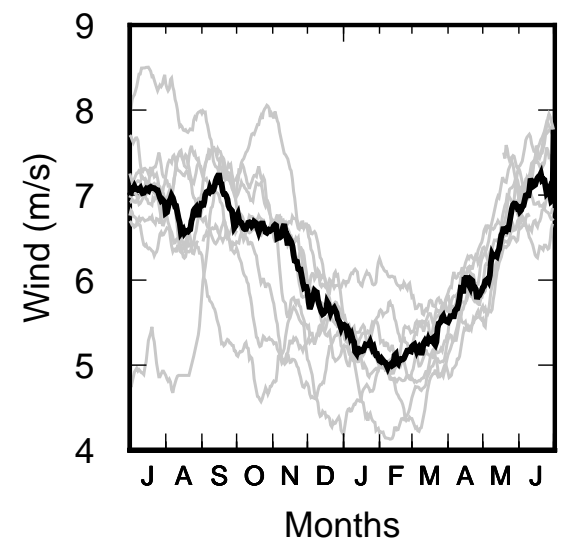

Figure 5.4: Yearly trend of meteorological datasets, smoothed with a 90-day moving window. Individual years of data are plotted from July to July of the following year (grey lines) with the median calculated (black lines). (a) Atmospheric pressure. (b) Rainfall. (c) Temperature. (d) Wind speed.

thermoelastic strain in the upper crust (Ben-Zion and Leary, 1986; Prawirodirdjo et al., 2006). While surface temperature variations only influence the very upmost crustal layer, Ben-Zion and Leary (1986) demonstrated that this alone can induce thermo-elastic strains down to depths on the order of the surface temperature wavelength. In the case of an annual cycle, strain perturbations are expected to be delayed by $2-3$ months following the initial surface temperature variation (Ben-Zion and Leary, 1986; Tsai, 2011). Wind speed related changes, on the other hand, reflect systematic noise excitation through interaction with topography, vegetation or infrastructure (Hillers and Ben-Zion, 2011; Hillers et al., 2015). The resulting influence on velocity changes is therefore expected to be instantaneous. Thus, although the two datasets correlate in time, the velocity response to either one should be different.

We cross-correlate meteorological datasets with onshore velocity changes to investigate a possible relationship between annual signals. Time series are first demeaned and detrended, before cross-correlating the data between 1 January 2009 and 1 June 
2016. This looks to exclude earthquake-induced changes (Section 4.3.3). A delay time is then picked manually from the cross-correlation function, based on the lag time that records highest amplitude peaks, and the correlation coefficient calculated after shifting the meteorological data by this delay. A positive delay time corresponds to a delayed velocity response relative to meteorological data. A correlation coefficient above zero $(\mathrm{CC}>0)$ indicates a positive linear relationship while a correlation coefficient below zero $(\mathrm{CC}<0)$ indicates a negative relationship.

Figure 5.5 shows the results of cross-correlating rainfall, temperature and wind speed datasets with 1.0-2.0 Hz onshore single-station velocity changes, which demonstrate clear seasonality. Atmospheric pressure changes are not shown, with no clear influences on velocities at higher frequencies. All three mechanisms - rainfall, temperature and wind speed changes - remain plausible after cross-correlating the datasets, perhaps suggesting a collective influence. Temperature changes show a positive correlation with velocities $(\mathrm{CC}>0.6)$ while wind speed changes show a negative correlation $(\mathrm{CC}<-0.5)$. Velocity changes recorded by onshore cross-components are better represented by a temperature induced change, with a delay time of approximately 25 days (Fig. 5.5d). This is slightly shorter than expected for thermally induced changes, though similar delays have been found elsewhere that are considered to potentially reflect this mechanism (Meier et al., 2010; Hillers et al., 2015). Auto-correlations, conversely, are better represented by wind-induced changes, with a near-zero delay time recorded (Fig. 5.5f). This again could reflect the sensitivity of auto-correlations to changes in the noise wavefield. Significant correlations with rainfall are also observed $(\mathrm{CC} \leq-0.5)$, with the negative relationship consistent with the filling of open pore space following significant precipitation events (Sens-Schönfelder and Wegler, 2006). Delay times of 1-2 months are larger than might be expected (Fig. 5.5b), perhaps reflecting that annual rainfall cycles are not a primary driver of seasonal velocity changes. In support of this, delay times are found to approach zero when smoothing is reduced (Fig. 5.6), indicating that isolated, short-term, heavy rainfall events may be more important in terms of rainfall induced changes onshore.

Annual velocity changes recorded by $0.1-1.0 \mathrm{~Hz}$ onshore single-stations vary from those recorded by 1.0-2.0 Hz datasets, with peak velocities occurring 2-3 months earlier in the cross-components (Fig. 5.3b). Identifying the underlying mechanism behind these changes in particular could be useful for discriminating between volcanic and non-volcanic sources, given observed similarities with velocities recorded by $0.1-1.0 \mathrm{~Hz}$ cross-components at White Island (Fig. 4.13). We perform a same analysis as before, cross-correlating $0.1-1.0 \mathrm{~Hz}$ velocity changes with meteorological 
(a) Rainfall Comparison

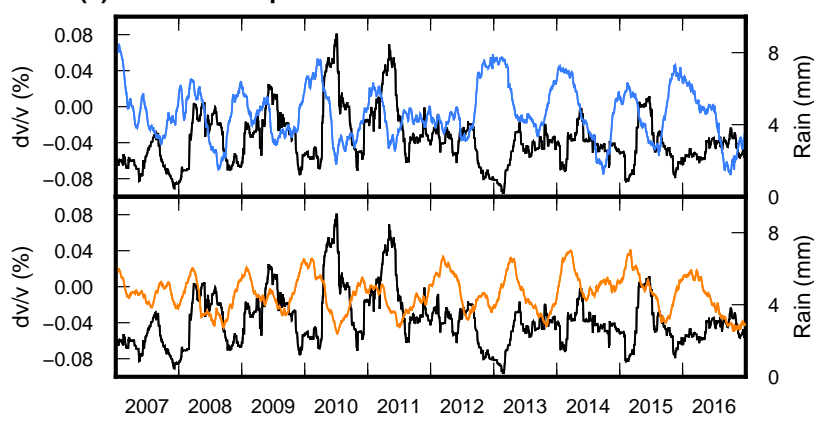

(c) Temperature Comparison

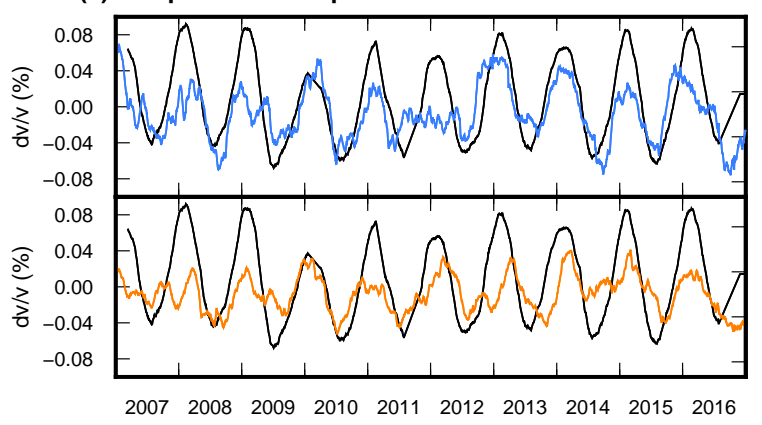

(e) Wind Speed Comparison

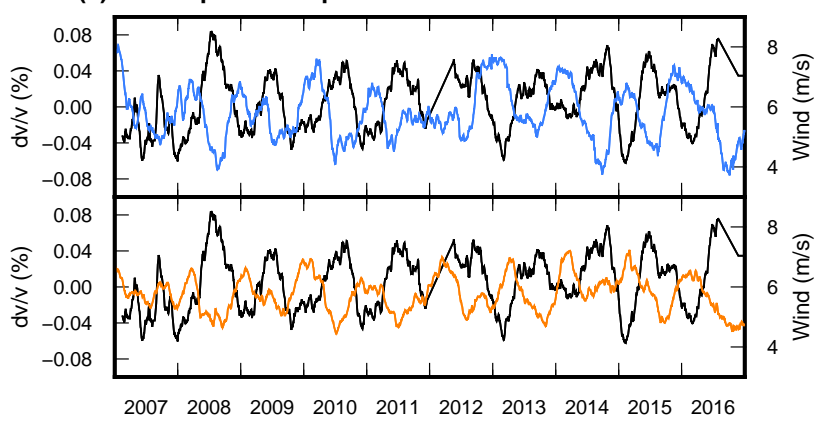

(b)

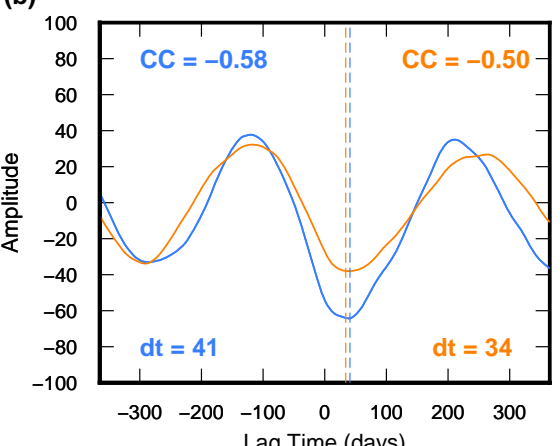

(d)

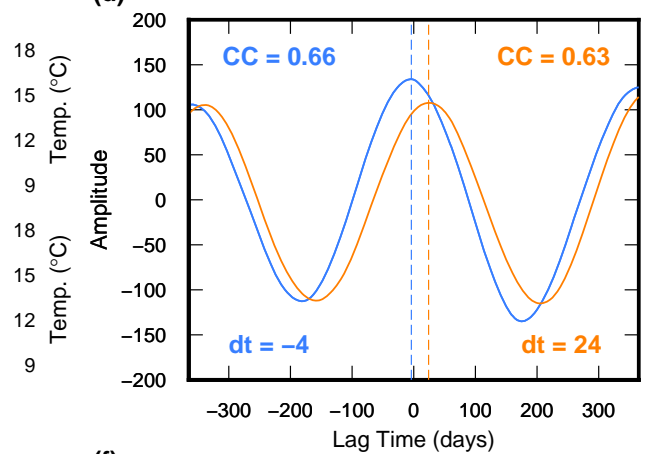

(f)

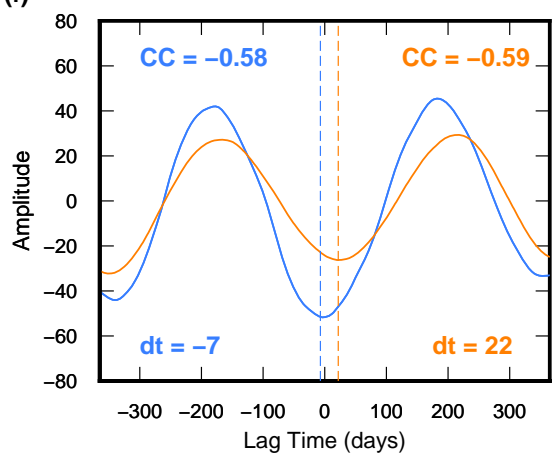

Onshore (SC, $1.0-2.0 \mathrm{~Hz}$ )

Figure 5.5: Comparison between 90-day smoothed 1.0-2.0 $\mathrm{Hz}$ single-station onshore velocity changes (average of HAZ, MWZ and PUZ stations) with meteorological datasets. Velocity changes from auto-correlations (AC) and cross-components (SC) are denoted by the key (blue and orange respectively) while meteorological data is shown as a black line, also smoothed by 90-days. Values for the correlation coefficient (CC) and delay times (dt) are shown, coloured based on the corresponding velocity dataset, with a dashed line representing the chosen pick. (a) Rainfall comparison. (b) Rainfall CC and dt. (c) Temperature comparison. (d) Temperature CC and dt. (e) Wind speed comparison. (f) Wind speed CC and dt.

datasets in Figure 5.7. Auto-correlations do not show a clear relationship with any of the meteorological datasets, with low values of $\mathrm{CC}$ for temperature and wind speed comparisons (Fig. 5.7d and 5.7f) and a larger than expected delay time with rainfall (Fig. 5.7b). The cross-components however show good fits with rainfall and wind speed changes (Fig. 5.7b and 5.7f). A correlation with wind speed is unexpected given minimal evidence of wind induced changes in 1.0-2.0 Hz onshore 
cross-components (Fig. 5.5) and the reduced influence of wind at depth (Young et al., 1994). However, we note that the yearly trend of 0.1-1.0 Hz cross-component velocities, peaking in December-January (Fig. 5.3b), is a good fit for the yearly rainfall lows (Fig. 5.4b). The effect of rainfall induced peaks in December - January combined with possible temperature induced peaks in February - April, evidenced in $1.0-2.0 \mathrm{~Hz}$ cross-components (Fig. 5.5b), might then be expected to produce an artificially good fit with the lowest wind speeds in January-March. Analysis of individual years of meteorological datasets and subsequent velocity changes is recommended to investigate this further.

Identifying the controlling environmental influence in velocity changes has proved to be somewhat ambiguous. Temperature induced thermo-elastic strains appear to be the most likely mechanism - of those investigated — driving seasonal velocity changes in 1.0-2.0 Hz onshore cross-components, while there is evidence to support wind speed changes causing systematic bias in 1.0-2.0 Hz auto-correlations (Fig. 5.5). In 0.1-1.0 Hz onshore cross-components, a good fit was found with rainfall and wind speed changes; though, we consider wind speed changes unlikely given the lack of evidence of wind speed changes at 1.0-2.0 Hz and expected depth sensitivities. Instead, we favour a possible combination of temperature and rainfall induced changes. One aspect potentially obscuring source identification relates to the spatial characteristics of the meteorological processes. Stations used to record onshore velocity changes - HAZ, MWZ and PUZ stations - are all located approximately

(a) Rainfall Comparison (30-day-smooth)

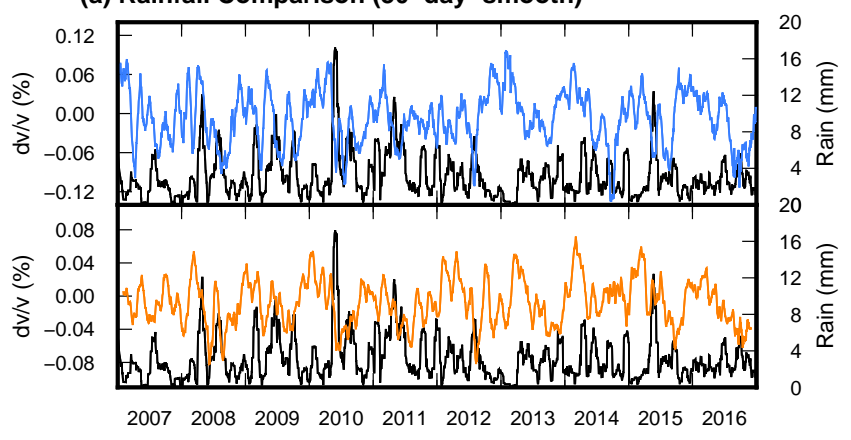

Onshore $(\mathrm{AC}, 1.0-2.0 \mathrm{~Hz})$ (b)

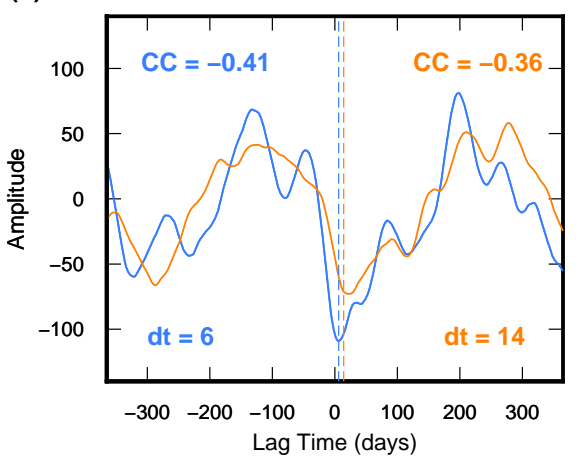

Onshore (SC, $1.0-2.0 \mathrm{~Hz})$

Figure 5.6: Comparison between 30-day smoothed 1.0-2.0 Hz single-station onshore velocity changes (average of HAZ, MWZ and PUZ stations) with rainfall. Velocity changes from auto-correlations (AC) and cross-components (SC) are denoted by the key (blue and orange respectively) while meteorological data is shown as a black line, also smoothed by 30-days. Values for the correlation coefficient (CC) and delay times (dt) are shown, coloured based on the corresponding velocity dataset, with a dashed line representing the chosen pick. (a) Rainfall comparison. (b) Rainfall CC and dt. 
(a) Rainfall Comparison

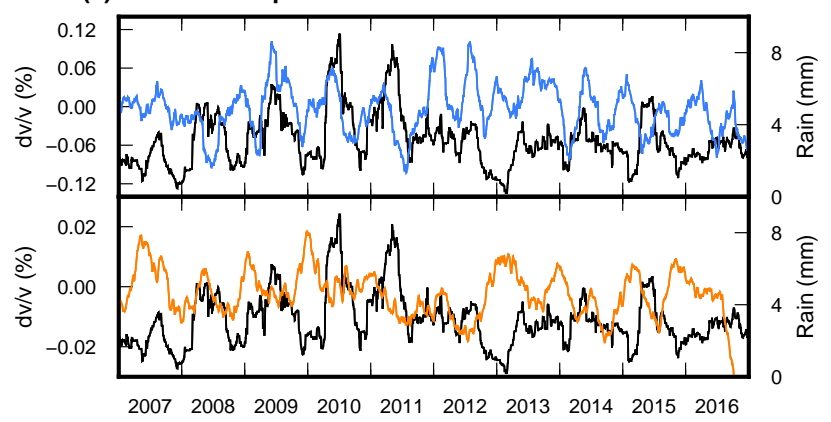

(c) Temperature Comparison

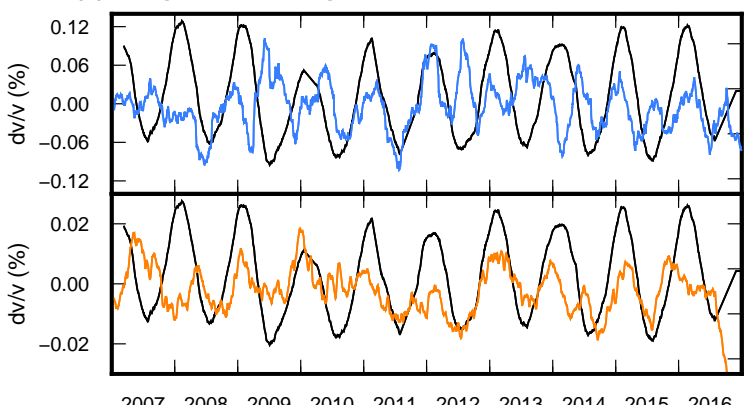

(e) Wind Speed Comparison

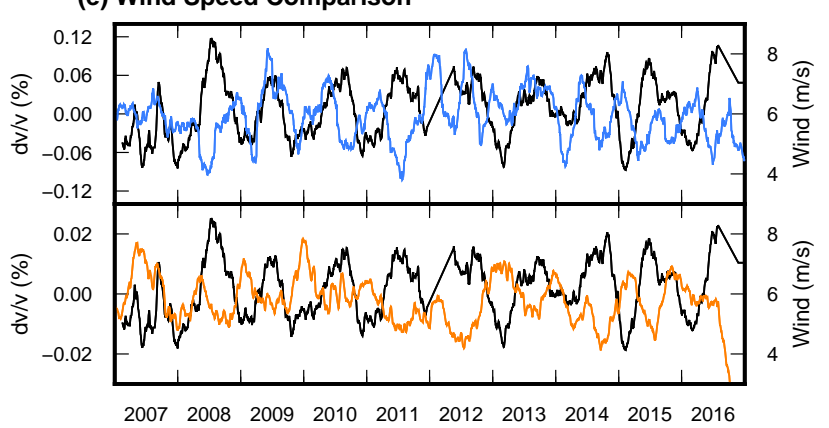

$2007200820092010 \quad 20112012 \quad 2013 \quad 2014 \quad 20152016$ (b)

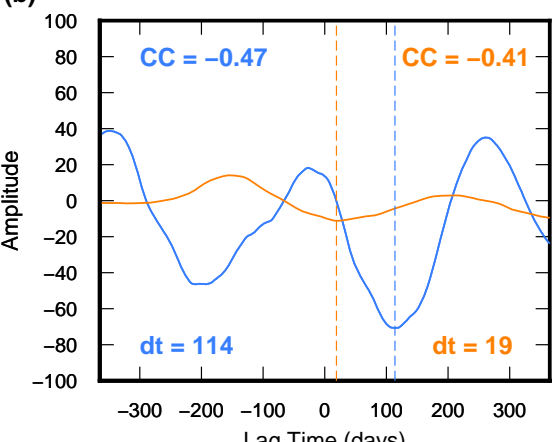

(d)

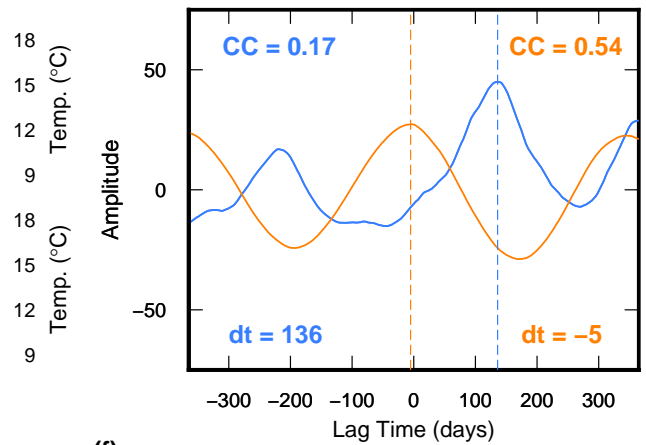

(f)

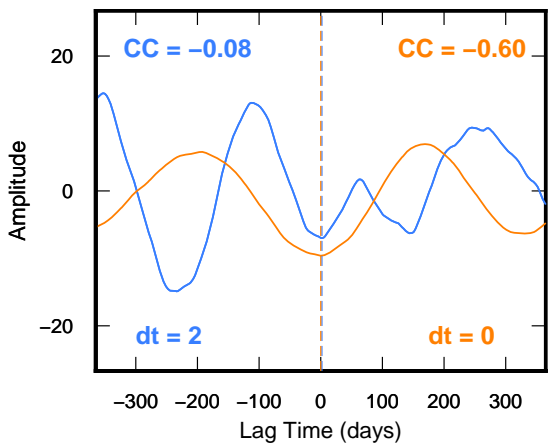

Onshore (SC, $0.1-1.0 \mathrm{~Hz})$

Figure 5.7: Comparison between 90-day smoothed 0.1-1.0 Hz single-station onshore velocity changes (average of HAZ, MWZ and PUZ stations) with meteorological datasets. Velocity changes from auto-correlations (AC) and cross-components (SC) are denoted by the key (blue and orange respectively) while meteorological data is shown as a black line, also smoothed by 90-days. Values for the correlation coefficient (CC) and delay times (dt) are shown, coloured based on the corresponding velocity dataset, with a dashed line representing the chosen pick. (a) Rainfall comparison. (b) Rainfall CC and dt. (c) Temperature comparison. (d) Temperature CC and dt. (e) Wind speed comparison. (f) Wind speed CC and dt.

50-100 km from weather stations used to acquire meteorological data. Locational differences may therefore be responsible for mixed results. A more complete analysis of environmental factors is therefore recommended, incorporating additional onshore stations and more localised meteorological datasets. In the absence of a more complete understanding of velocity responses to seasonal influences, we look to distinguish between environmental and volcanic signatures by directly comparing 
the character of velocity changes measured onshore and those measured at White Island.

\subsubsection{Tectonic Earthquakes}

Earthquake induced seismic velocity changes are well-established, with numerous examples of co-seismic and post-seismic deformation detected using ambient noise (e.g. Brenguier et al., 2008a; Hobiger et al., 2012; Minato et al., 2012; Takagi et al., 2012; Froment et al., 2013; Brenguier et al., 2014; Lesage et al., 2014; Taira et al., 2015; Heckels et al., 2018). The precise source of earthquake induced velocity changes remains contentious, with full discussion outside the scope of this thesis. However, we broadly consider whether co-seismic velocity decreases observed at White Island (Fig. 4.14) reflect static or dynamic stress changes and whether changes following the $2008 M_{W} 5.2$ earthquake (Fig. 4.14f) reflect a post-seismic relaxation process.

Static stress changes occur as a direct result of slip on a fault, with the surrounding region subjected to both compressional and dilatational deformation (King et al., 1994). Velocity increases are expected in dilatational environments - through the opening of microcracks - and velocity decreases in compressional environments - through the closing of microcracks (Nur and Simmons, 1969; Yamamura et al., 2003). Conversely, dynamic stress changes are associated with the passage of seismic waves (Belardinelli et al., 2003). These can cause semi-permanent damage to shallow crustal layers during strong ground motion (e.g. Takagi et al., 2012; Taira et al., 2015), with a velocity decrease expected.

Two earthquakes are identified as sources of significant velocity decreases. The largest of these, a $M_{W} 7.1$ earthquake in September 2016, produced a significant velocity decrease of $0.05-0.1 \%$ at both onshore and White Island seismic stations (Fig. 4.14). Dynamic stress changes are considered the most likely candidate for velocity decreases, given large offsets - approximately $200 \mathrm{~km}$ - between seismic stations and the earthquake's epicenter (Fig. 4.14a). This is supported by static Coulomb stress changes computed for this event, with no significant changes calculated beyond approximately $50 \mathrm{~km}$ of the epicenter Warren-Smith et al. (2018). The smaller earthquake, a $M_{W} 5.2$ earthquake in June 2008, produced a velocity decrease of $0.1 \%$ at WIZ station on the volcano (Fig. 4.14f). Discriminating between static and dynamic stress changes here is more complicated given the close proximity to the volcano (Fig. 4.14e). However, co-seismic velocity changes observed in 1.0-2.0 $\mathrm{Hz}$ single-station results at the volcano (Fig. 4.10a), which is sensitive to shallow layers, and a similar style of velocity decrease to the $M_{W}$ 7.1 Te Araroa earthquake suggest dynamic stress changes are the more likely cause. 
A long-term velocity increase is observed directly following the $M_{W} 5.2$ earthquake in 2008, as demonstrated by $0.1-1.0 \mathrm{~Hz}$ cross-components at WIZ station in Figure 5.8a. We consider whether this increase reflects post-seismic relaxation, commonly associated with the healing of cracks following co-seismic deformation (Hiramatsu et al., 2005; Li et al., 2006), or whether it reflects a long-term volcanic signal that was momentarily interrupted by a co-seismic velocity decrease. Post-seismic relaxation commonly follows a logairithmic trend and has been observed on a wide range of time scales (e.g. Schaff and Beroza, 2004; Brenguier et al., 2008a; Lesage et al., 2014; Taira et al., 2015; Heckels et al., 2018). In Figure 5.8b, we model the post-seismic velocity increase in Figure 5.8a as both a logarithmic and linear trend through linear regression. For a logarithmic curve, we solve for $a$ and $b$ in $d v / v=a+b \log _{10}(d)$ where $d$ is the number of days since the earthquake. Solving the linear equivalent then simply involves removing the logarithmic term $(d v / v=a+b d)$. Only velocity changes up to 1 January 2012 are used in the linear regression analysis, thus excluding any changes resulting from volcanic activity in 2012-2013. A logarithmic trend appears to produce a better fit with the velocity increase in Figure 5.8b, with the linear trend not representative of more rapid velocity increases in the first 6 months following the earthquake. Additionally, velocities begin to plateau at approximately pre-earthquake levels by 2011, staying this way until eruptive activity begins late2012. Velocities from 2013 onwards however appear more consistent with a linear trend starting mid-2008.

We take this opportunity to further consider the similarities between 90-day smoothed velocity changes recorded at White Island and onshore (Fig. 5.8a). If these changes are reflective of an environmental source process, performing a correction could be crucial for highlighting non-meteorological effects. For example, in Figure 5.8a we see that local peaks at the start and end of 2009, which contribute to the fit of a logarithmic trend, coincide with increases onshore. We therefore perform a straightforward correction by subtracting onshore velocity changes - smoothed by 90-days - from velocities recorded at WIZ station prior to smoothing. The purpose here is to illustrate potential ambiguity when interpreting temporal velocity changes based on a single source process, acknowledging that this is a simplified approach to correcting for seasonal changes. In Figure 5.8c, seasonally corrected velocity changes are shown with a newly modelled logarithmic and linear fit. This time, velocity changes appear much more linear in their increase following the earthquake due to the reduction of velocity peaks in early- and late-2009. Additionally, instead of plateauing in 2011, the velocity continues to increase at a rate that also fits with the long-term trend during eruptive activity. Thus if using the corrected time series, 
it could be interpreted that velocity changes resulting from the earthquake are almost entirely coseismic. Distinguishing between these two end members is difficult without additional data pre-earthquake and highlights the difficulty associated with monitoring when velocity changes are induced by multiple sources. However, in the absence of a more complete analysis of seasonal influences at the volcano, we consider post-seismic relaxation a more likely candidate for velocity changes during this period . This is consistent with velocity responses observed elsewhere following large

(a) Cross-components (SC), $0.1-1.0 \mathrm{~Hz}$

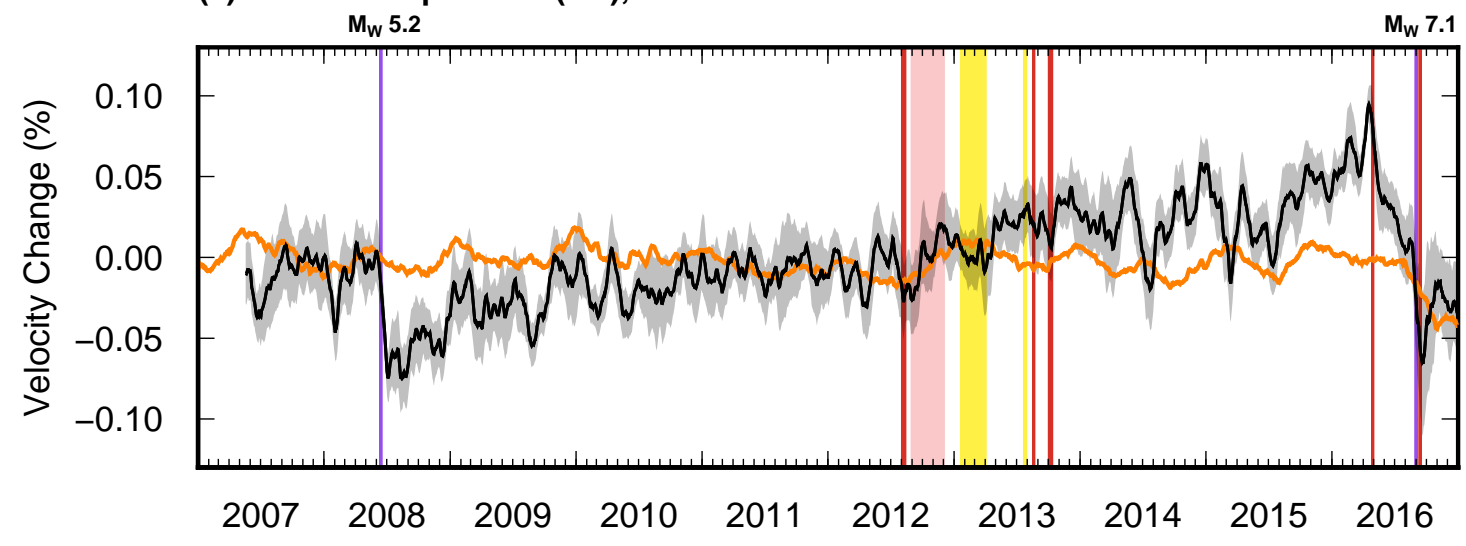

(b) No Correction

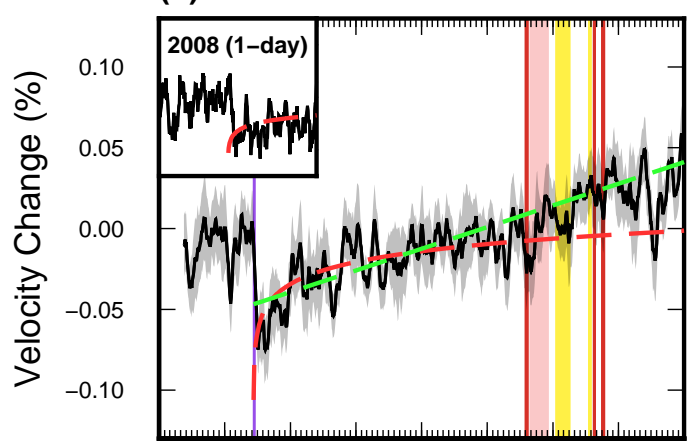

20072008200920102011201220132014 (c) Corrected (WIZ - Onshore)

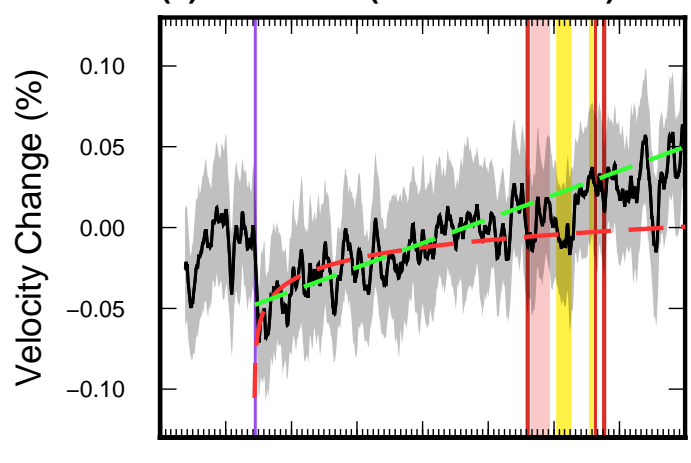

20072008200920102011201220132014

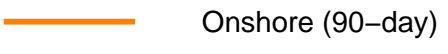

Linear fit

Figure 5.8: Analysis of post-seismic relaxation - associated with $M_{W} 5.2$ - before and after applying a seasonal correction. (a) 30-day smoothed velocity changed recorded by $0.1-1.0 \mathrm{~Hz}$ cross-components at WIZ station, with seismic and volcanic activity highlighted (see key). Also shown are 90-day smoothed changes at onshore stations (HAZ, MWZ, PUZ) representing seasonal change. (b) Post-seismic response is modelled by logarithmic and linear curves through linear regression analysis. (c) Post-seismic response is again modelled, but this time after applying a seasonal correction by subtracting onshore 90-day velocity changes from measurements made at WIZ station. 
tectonic earthquakes, both in volcanic (e.g. Lesage et al., 2014) and non-volcanic settings (e.g. Brenguier et al., 2008a; Hobiger et al., 2012; Minato et al., 2012; Heckels et al., 2018).

Finally, we also observe possible evidence of post-seismic deformation following the $M_{W} 5.2$ earthquake. In Figure 5.9, velocity changes recorded by $0.1-1.0 \mathrm{~Hz}$ autocorrelations and cross-components are compared. Clear similarities are observed in the long-term trend. However, where velocity changes recorded by cross-components show a coseismic decrease associated with the nearby $M_{W} 5.2$ earthquake, the velocity response of auto-correlations appears spread over the 3-4 months following. Similar behaviour has been observed by Froment et al. (2013), who equally found post-seismic velocity changes at longer periods - 12-20 seconds — following a $M_{W}$ 7.9 earthquake. They attributed this behaviour to post-seismic slip, while also considering the possibility of a visco-elastic relaxation in the deep crust. Observed differences in deformation style between auto-correlations and cross-components then may suggest the former are resolving velocity changes at greater depth. This is supported by the strong similarities pre-2013 between $0.1-1.0 \mathrm{~Hz}$ cross-components and 1.0-2.0 Hz auto-correlations (Figure. 4.10a).

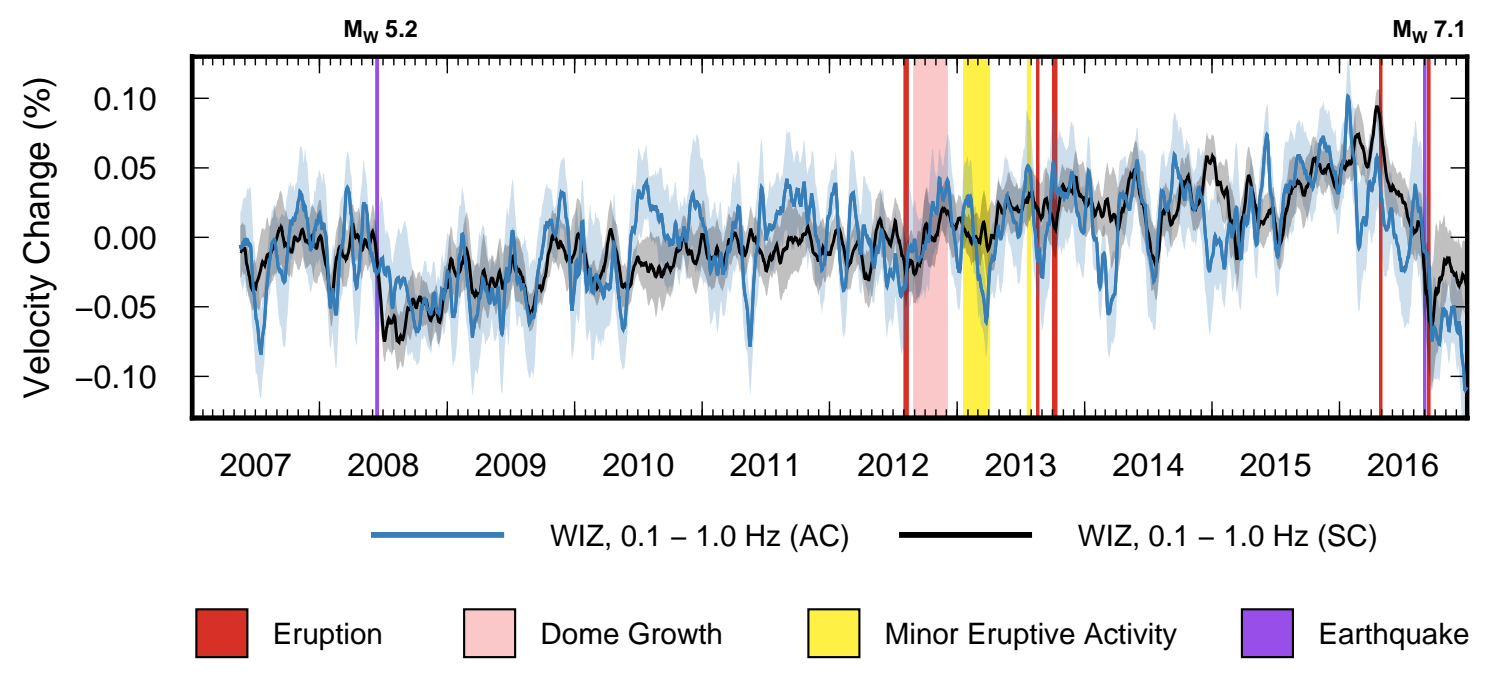

Figure 5.9: Velocity changes recorded by $0.1-1.0 \mathrm{~Hz}$ auto-correlations and crosscomponents at WIZ (smoothed by 30-days), with evidence of post-seismic deformation following a $M_{W} 5.2$ earthquake in auto-correlations. 


\subsection{Volcanic Changes at White Island Volcano}

A primary objective of this thesis is to investigate the use of ambient noise to understand volcanic processes at White Island. We close the discussion by reviewing some of the observed changes up to now and consider how these aid in our understanding of behaviour at the volcano. This includes: (1) decorrelation before and during volcanic activity, (2) long-term velocity increases and apparant changes in 2016, and (3) the potential for real-time monitoring. It is important to stress here that discussion cannot realistically include all potentially significant observations. The exploratory nature of the thesis has produced a wide variety of observations, with a substantial amount of time going into the comprehensive analysis of various datasets. Focus is therefore given to results we feel are more reliable and, where possible, justification is provided for the exclusion of data from further discussion.

\subsubsection{Decorrelation Before and During Volcanic Activity}

In addition to seismic velocity changes we also calculated the correlation coefficient between current and reference stacks. This can be used as an additional monitoring tool, with the decorrelation of cross-correlation functions potentially associated with changes in the scattering of seismic energy resulting from structural changes. We observe significant changes in measured correlation coefficients in the vicinity of volcanic activity, especially in $1.0-2.0 \mathrm{~Hz}$ single-station results at WIZ during 2012-2013 eruptive activity (Fig. 4.9). However, given that the correlation coefficient is also used as a quality control check, changes based on temporal variations in the quality of recorded noise need to be ruled out. This section therefore considers whether observed changes in the correlation coefficient are related to a structural change or related to reduced quality of computed cross-correlation functions.

Volcano-seismicity is a potential source of contamination at White Island, with the potential to reduce the coherence between current cross-correlation functions and reference stacks. Unlike tectonic-related seismicity, which occurs through shear failure, volcano-seismicity is commonly associated with fluid processes. Low-frequency earthquakes and volcanic tremor are common manifestations of volcano-seismicity (McNutt, 2005), often preceding or accompanying volcanic eruptions (Chouet, 2003). The precise source process for these events remains a controversial topic and is outside the scope of this thesis.

The occurence of volcanic seismicity at White Island is well documented, with dominant frequencies above $1 \mathrm{~Hz}$ (Sherburn et al., 1998; Chardot et al., 2015; Jolly et al., 2016). One of the better methods for monitoring volcano-seismicity is through real- 
(a)

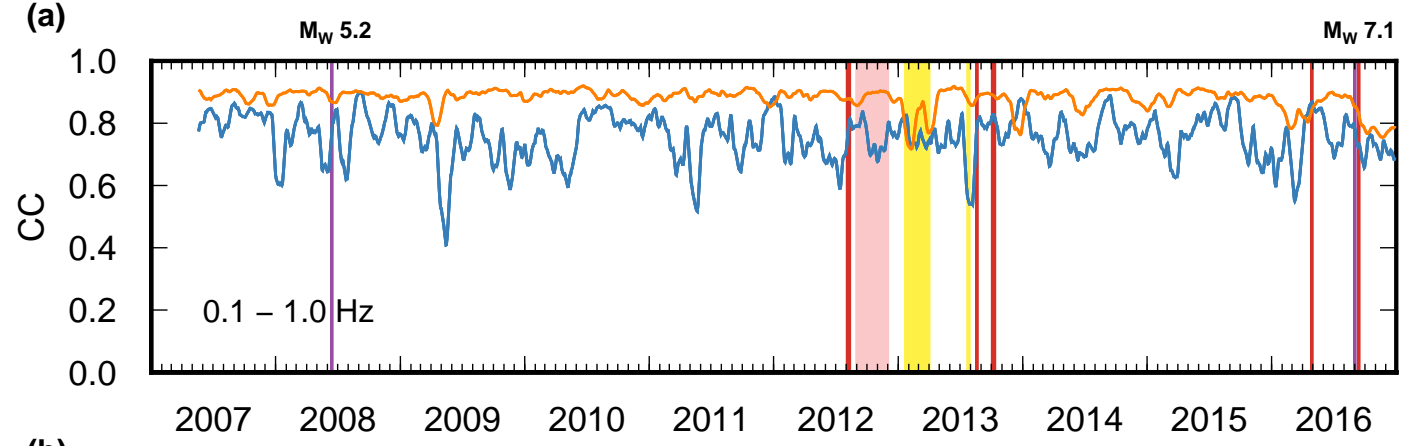

(b)

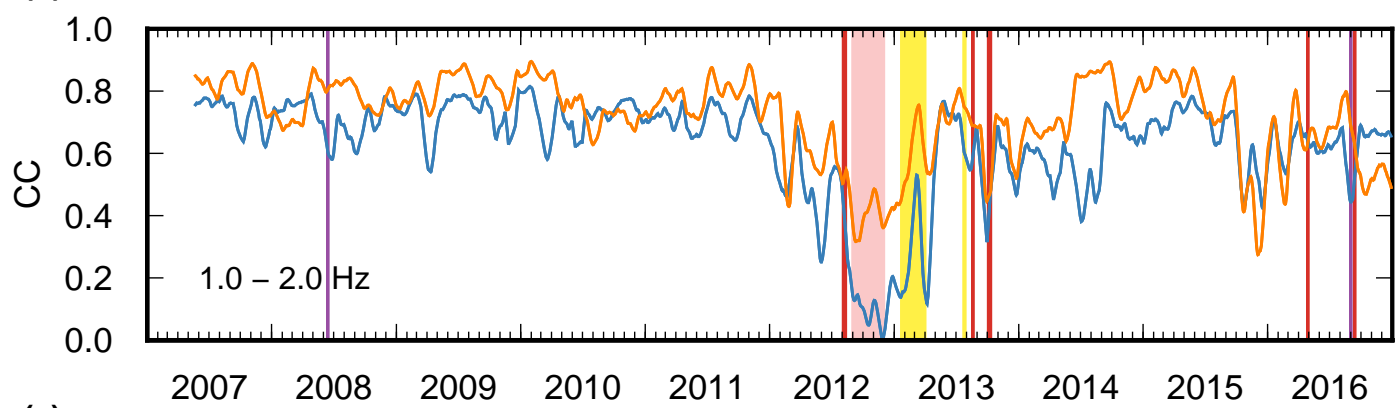

(c)

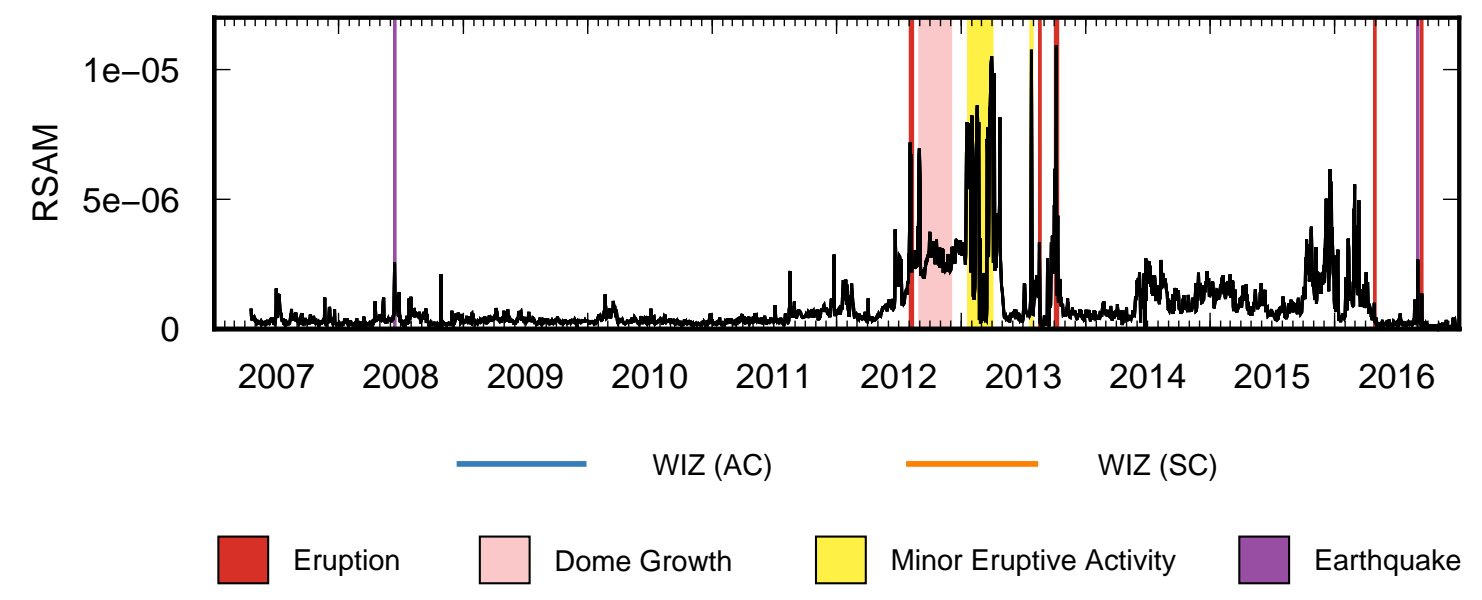

Figure 5.10: Comparison between single-station correlation coefficients (CC) and RSAM $(1.2-5.0 \mathrm{~Hz})$ measured at WIZ station. Correlation coefficients are smoothed with a 30-day moving-window. Both auto-correlation (blue) and cross-component (orange) data are shown. (a) 0.1-1.0 Hz CC (b) 1.0-2.0 Hz CC (c) Vertical component RSAM.

time seismic amplitude measurements (RSAM). This corresponds to measures of the average seismic amplitude recorded by a station in 1-minute intervals (Endo and Murray, 1991). We present RSAM data recorded by the vertical component of WIZ station during the period of study (Fig. 5.10c). This has been bandpass filtered between 1.2 and $5.0 \mathrm{~Hz}$ and the RMS amplitude computed for 1-minute intervals. We further calculate the mean over each day to give the same sampling interval as velocity changes. Daily RSAM measurements between 2007-2017 can then be used to quantify volcano-seismicity during the study period. 
RSAM is compared with 1.0-2.0 Hz correlation coefficients measured at WIZ station - by both auto and cross-components - in Figure 5.10b. The timing of reduced correlation coefficient measurements in 2012-2013 are found to coincide with a period of increased RSAM activity. Near-zero measurements for auto-correlations indicate there is no relationship between individual 10-day cross-correlation functions and the reference stack during this period. Structural changes are not likely to produce a change of this magnitude, especially not with subsequent recovery. Thus, changes are more likely related to contamination via volcano-seismicity, which operates at similar frequencies. The case is similar for RSAM increases late-2015 and early-2016, with decreases in the correlation coefficient observed for both auto-correlations and cross-components. Decreases following the April 2016 eruption, however, cannot be easily related to volcano-seismicity, with low RSAM levels during this period. Decorrelation during this time is therefore unlikely to have resulted from contamination by volcano-seismicity.

Decreases in the cross-correlation coefficient are also observed in 0.1-1.0 auto-correlations and cross-components at WIZ (Fig. 5.10a). Changes recorded by the cross-components are the most significant, where decreases in early 2013 and in the months before the April 2016 eruption are large relative to background changes. For 0.1-1.0 Hz seismic noise, contamination of the cross-correlation functions is unlikely given that tremor is typically observed above $1 \mathrm{~Hz}$ at White Island. This is supported by the lack of a correlation coefficient decrease during heightened RSAM levels in 2012. We therefore consider that declines in the correlation coefficient - observed by $0.1-1.0 \mathrm{~Hz}$ cross-components - could represent structural changes within the volcanic system. Such decreases could then be related to the deformation driving volcano-seismicity during active periods at the volcano. However, we exercise caution with this interpretation, with processing of RSAM data between $0.1-1.0 \mathrm{~Hz}$ required completely rule out potential contamination from volcano seismicity.

\subsubsection{Long-term Velocity Increases and Apparant Changes in 2016}

In this section we discuss the long-term velocity increase recorded at WIZ station between 2007 and 2017, best observed in the results of $0.1-1.0 \mathrm{~Hz}$ single-station ambient noise (e.g. Fig. 4.7). The start of this increase coincides with a $M_{W} 5.2$ earthquake in 2008 within $10 \mathrm{~km}$ of White Island (Fig. 4.14e). Whether a similar trend is present before this event is uncertain without extending the dataset back further in time. In this section, we describe the evolution of this increase in six 
stages based on visual differences through time (Fig. 5.11a-f). We focus on velocity changes recorded by $0.1-1.0 \mathrm{~Hz}$ cross-components at WIZ station for this purpose, with auto-correlations observed to be less stable (e.g. Fig. 4.8). This could relate to the lack of spectral whitening when processing auto-correlations. Correlation coefficient and RSAM measurements are also shown for comparison.

The first two stages of the long-term velocity trend (Fig. 5.11a and 5.11b) cover the response following a co-seismic velocity decrease associated with the $M_{W} 5.2$ earthquake in 2008. Visually, velocity changes resemble a post-seismic relaxation process in the two and a half years following this event (Fig. 5.11a) before plateauing at approximately pre-earthquake levels (Fig. 5.11b). This remains unchanged until volcanic activity begins mid-2012. Potential ambiguity in the interpretation of post-seismic relaxation was discussed in Section 5.2.2, relating to whether velocity changes reflect a logarithmic healing process or the continuation of a linear trend. We consider post-seismic relaxation a more likely candidate, consistent with postseismic velocity responses observed elsewhere following large tectonic earthquakes (e.g. Brenguier et al., 2008a; Hobiger et al., 2012; Minato et al., 2012; Lesage et al., 2014; Heckels et al., 2018).

Volcanic activity in 2012-2013 is accompanied by an increase in the seismic velocity (Fig. 5.11c), suggesting a volcanic origin. This was a particular active period for White Island, with well-documented increases in gas flux - $\mathrm{SO}_{2}$ and $\mathrm{H}_{2} \mathrm{~S}$ in particular - and volcanic tremor (Chardot, 2015). The strong match between the timing of volcano-seismicity, represented by vertical-component RSAM data, increases in seismic velocity, and the timing of eruptive activity suggests these datasets are inter-related. There is some ambiguity associated with the start time of this velocity increase, with velocity changes recorded by onshore stations also increasing during this period (e.g. Fig. 4.13 and 5.8a). However, onshore velocities begin to decrease in early-2013 whereas velocities at WIZ station continue to increase. This deviation from a seasonal trend is clearly observed when comparing velocity changes in 20122013 to the yearly seasonal trend of onshore velocities recorded by cross-components (Fig. 5.12c). Using this, we argue that the further velocity increase from March 2013 is of volcanic origin, supported by increases in volcano seismicity and decreases in the cross-correlation coefficient during this period (Fig. 5.11).

We interpret the velocity increase during eruptive activity in 2012-2013 to be the result of cracks closing under increased pressures beneath the volcano. This acknowledges that recent ambient noise work has shown volcanoes are capable of both positive and negative velocity changes depending on the relative position of the 

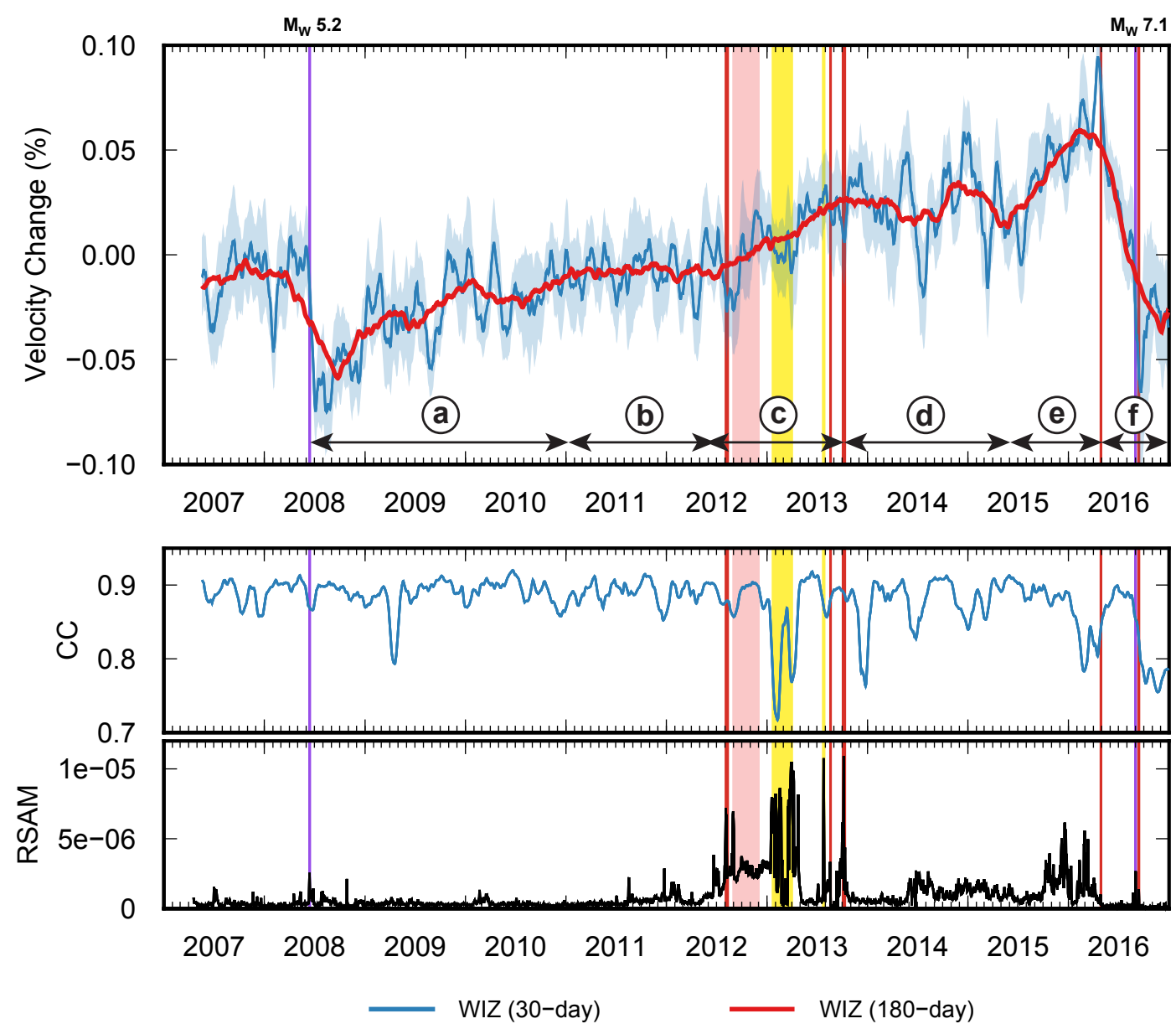

Eruption

Dome Growth

Minor Eruptive Activity

Earthquake

Figure 5.11: Long-term velocity increases at WIZ station are shown (180-day smooth) as recorded by $0.1-1.0 \mathrm{~Hz}$ cross-components between 2007-2017. For comparison, cross-correlation measurements are shown (30-day smooth) in addition to RSAM levels. Activity is characterised by six distinct periods, from (a) to (f), described in text.

source (e.g Anggono et al., 2012; Obermann et al., 2013; Budi-Santoso and Lesage, 2016; Donaldson et al., 2017). We therefore find no conflict with this interpretation relative to observations of velocity decreases during eruptive activity at other volcanoes globally (e.g. Brenguier et al., 2008b; Mordret et al., 2010). Determining an approximate source depth of this increase is difficult for two reasons. First, 0.1-1.0 $\mathrm{Hz}$ ambient noise is sensitive to both upper and middle crustal perturbations (Fig. 5.1). Second, the 1.0-2.0 Hz results are not as reliable during this period, with evidence that this dataset is contaminated by nearby volcano seismicity (Section 5.3.1). Therefore, without tighter depth control, we do not speculate on whether this change originates in the shallow hydrothermal system or is related to pressurisation of deeper magma chambers. 
(a)

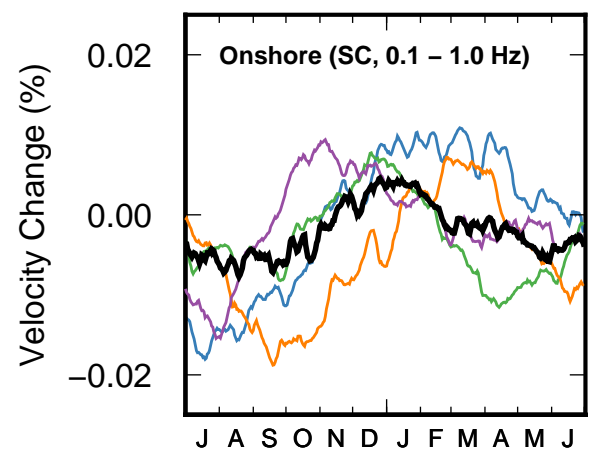

(b)

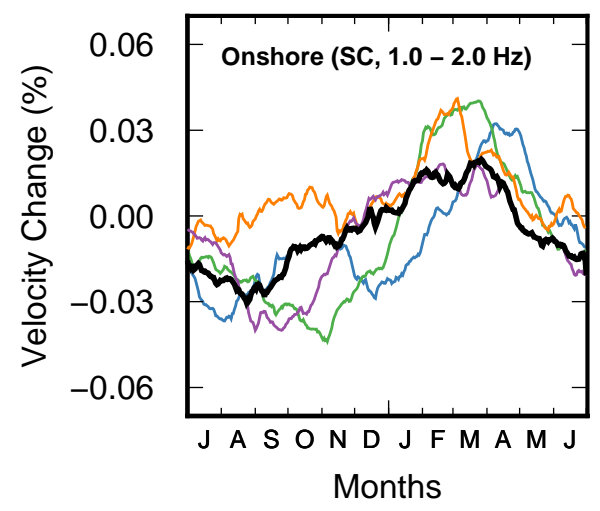

(c)

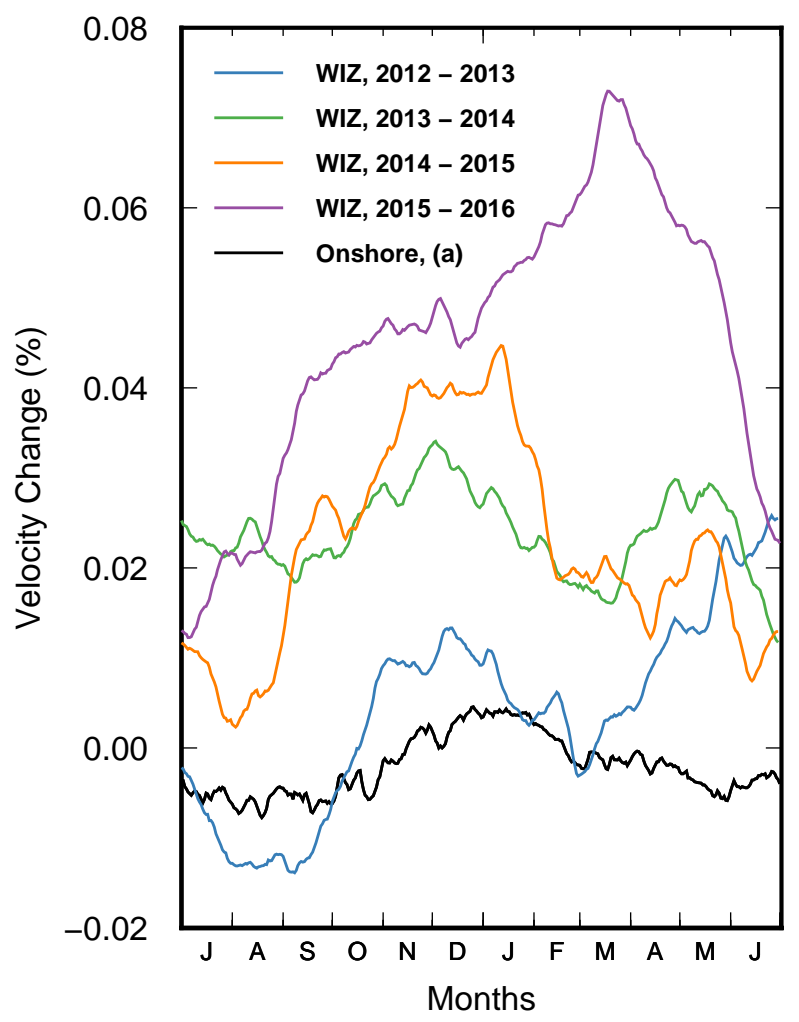

Figure 5.12: Seasonal influences at WIZ station are explored by comparing velocity changes to the yearly trend of velocities recorded by onshore cross-components (SC). Colors correspond to a single year of data recorded, reflecting the color scheme in (c). (a) 90-day smoothed 0.1-1.0 Hz onshore velocity changes. The black line represents the median yearly trend of velocity changes from Figure 5.3. Individual years of onshore changes are shown following the color scheme in (c). Years not included in this key are not shown, but still make up the median trend line. (b) 90-day smoothed 1.0-2.0 Hz onshore velocity changes. (c) The 0.1-1.0 Hz median yearly 0.1-1.0 Hz onshore velocity trend in (a) is compared with cross-component changes recorded during specific years at WIZ station.

Increased pressurization beneath a volcano might be expected to decline after an eruptive episode has ceased. However, velocities remain elevated relative to preeruption levels following activity in 2012-2013 (5.11d). This could indicate that any pressure source that developed during this period has not dissipated as a consequence of the October 2013 eruptions. Such an interpretation would then suggest the volcano remained in a heightened state of unrest following 2012-2013 activity. This provides a possible explanation for elevated RSAM amplitudes, above pre-2012 levels, continuing into 2014.

The long-term velocity remains relatively stable from 2014 to mid-2015, with smaller period of velocity increase observed between November 2014 and January 2015 (Fig. 5.11d). The timing and wavelength of this feature is similar to a peak recorded by 
onshore stations (e.g. Fig. 4.13 and 5.8a), though occurs 2-3 months earlier than the peak observed at WIZ station (Fig. 5.12a and 5.12c). Elevated RSAM levels during this period suggest the volcano was in a heightened state of unrest, thus it is possible then that this increase is associated with a pressurization event. However, given similarities with velocities recorded onshore, we do not feel there is a strong basis for this interpretation without further analysis of seasonal trends.

The build-up to the April 2016 eruption coincides with a rapid velocity increase starting mid-2015 (5.11e). This is slightly earlier than the expected seasonal trend (Fig. 5.12c), though velocities recorded by onshore stations at this time are also observed to increase (Fig. 5.12a). From December 2015 however, further increases cannot be linked to a seasonal feature (Fig. 5.12c). Additionally, decreases in the correlation coefficient from early-2016 are suggestive of a structural change at the volcano (Fig. 5.11). We therefore interpret these changes as a further pressurization event of the volcanic system, occurring from at least early-2016. Faster rates of increase, relative to 2012-2013, may be indicative of the explosive style of this eruption. An earlier start time is possible, with elevated RSAM levels from September-October 2015 (Fig. 5.11) and observations of crater floor uplift from mid-2015 (Hamling, 2017). We cannot however verify that velocity increases earlier than 2016 are related to these observations, given the similarities with onshore velocities at this time.

In the aftermath of the April 2016 eruption, the long-term velocity starts to decline rapidly. At the time of the $M_{W} 7.1$ Te Araroa earthquake in September 2016, which produced significant co-seismic velocity changes throughout the region (e.g. Fig. 4.14 and $5.9 \mathrm{~b}$ ), the velocity had fallen to levels slightly below pre-2015 velocities in the space of 2-3 months (Fig. 5.11f). Additionally, the correlation coefficient recovers immediately following the eruption and RSAM amplitudes fall to levels comparable to pre-2012 behaviour. We therefore interpret that these changes are reflective of a significant reduction in pressure levels underneath the volcano following the April-2016 eruption. It is unclear however whether the velocity would have continued decreasing to pre-2012 levels without the contribution of the Te Araroa earthquake.

Further eruptive activity in September 2016 could indicate that the volcano remained in a heightened state of unrest following the April eruption. However, a rapid decrease in velocity, a recovery of the correlation coefficient and reduced RSAM levels suggest otherwise. Additionally, measurements of gas flux remained at low levels (Hamling, 2017). The timing the September eruption is interesting, given it occurred only two weeks after the $M_{W} 7.1$ Te Araroa earthquake. Co-seismic velocity 
decreases from this event are considered the result of dynamic stress changes (Section 5.2.2) and can be thought to represent extensive damage to the crust. It is possible then that the September eruption was externally triggered by the $M_{W} 7.1$ earthquake.

A permanent change in the correlation coefficient is observed following the September 2016 eruption, which could be suggestive of structural damage. However, we consider this is likely representative of a sensor change at WIZ station. Unfortunately, this change was performed on the same day as the eruption, so distinguishing real structural change from an artificial change is difficult. We note though that the magnitude of this decrease - and subsequent non-recovery - is problematic in the context of what was a relatively minor eruptive event. Thus, we think this is an artificial change associated with a change in seismic sensor at WIZ station.

\subsubsection{Real-Time Monitoring}

The potential for real-time monitoring using ambient noise is an exciting prospect. There is little reason why future global volcano monitoring should not include ambient noise as an additional tool alongside more conventional monitoring techniques. In this final section we briefly consider what could have been gained had the results of this thesis been available in real time.

An ongoing problem in volcano monitoring is recognising when an eruption will occur, with the final system failure preceding one typically only recognised over short time-scales of days to minutes (Sparks, 2003). It is hoped that ambient noise can provide improved recognition of the final events leading up to an eruption, given a sensitivity to changes that may not be observable at the Earth's surface. In this study however, we have been unable to detect clear short-term changes that could have indicated imminent eruptive activity. This is not to say they do not exist, only that any suggestion of imminent activity would have been very difficult to interpret. This failure could represent limitations in the temporal resolution of velocity changes, with only minor internal or external triggers required to move a volcano into an eruptive state (Sparks, 2003). Alternatively, stress perturbations preceding eruptions may not be large enough to produce velocity changes above background fluctuations. Either way, the results of this thesis do not indicate ambient noise based monitoring would have improved short-term hazard forecasting at the volcano compared with alternative monitoring techniques (e.g. Chardot et al., 2015).

In the longer-term, we detect two periods of velocity increase interpreted to reflect the closing of cracks under increased pressures (Section 5.3.2). Up to now, we have 
revealed these changes by smoothing using a centered moving window, with this felt to best illustrate the true timing of velocity changes. For real-time monitoring however, smoothing is not possible using measurements that do not currently exist i.e. the second half of a centered window. The alternative then is to represent the velocity on a given day as the average of the previous $N$ days including itself (for an $N$-day moving window). This can introduce a delay on detectable changes and therefore limits the temporal resolution of velocity changes (Fig. 5.13). The greater the smoothing required to highlight changes, the larger this time shift will become. In Figure 5.13, this time shift is examined for 30-day and 180-day stacks during activity in 2012-2016. Velocity changes smoothed by 180-days in 'real-time' show a clear time delay of approximately 3 months i.e. half the window length. The velocity increase prior to the April 2016 eruption is still clearly visible however, showing a precursory change that deviates from the background trend in the months beforehand. We feel then that long-term velocity changes, discussed in Section 5.3.2, would have provided valuable information about the behaviour of the volcano alongside other geophysical monitoring tools.

It is becoming clear that a connection exists between large tectonic earthquakes and volcanic eruptions, with many cases of triggered volcanic eruptions reported globally (Linde and Sacks, 1998; Marzocchi, 2002; Manga and Brodsky, 2006). The distances between the initial earthquake and eruptive activity varies, but can be up

(a) 30-day smooth

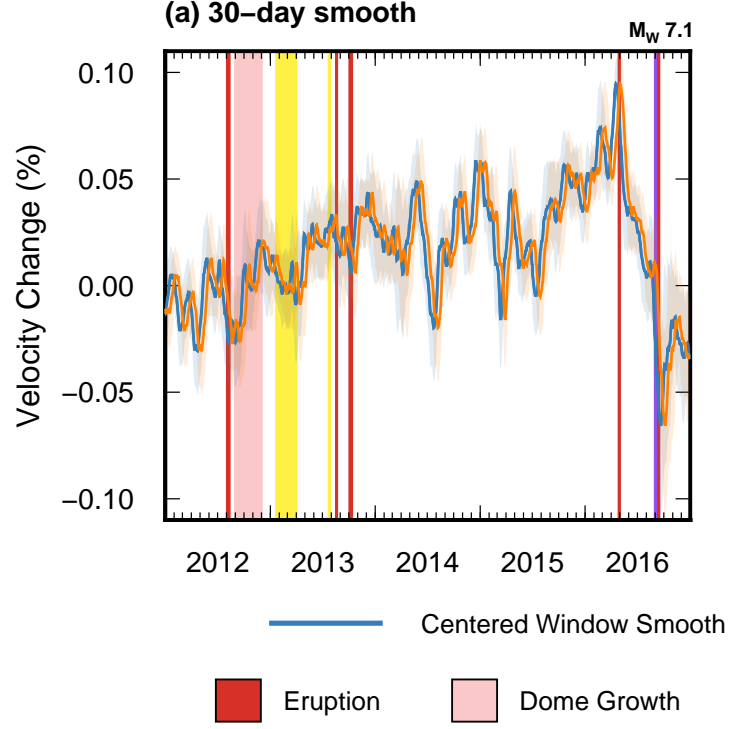

(b) 180-day smooth

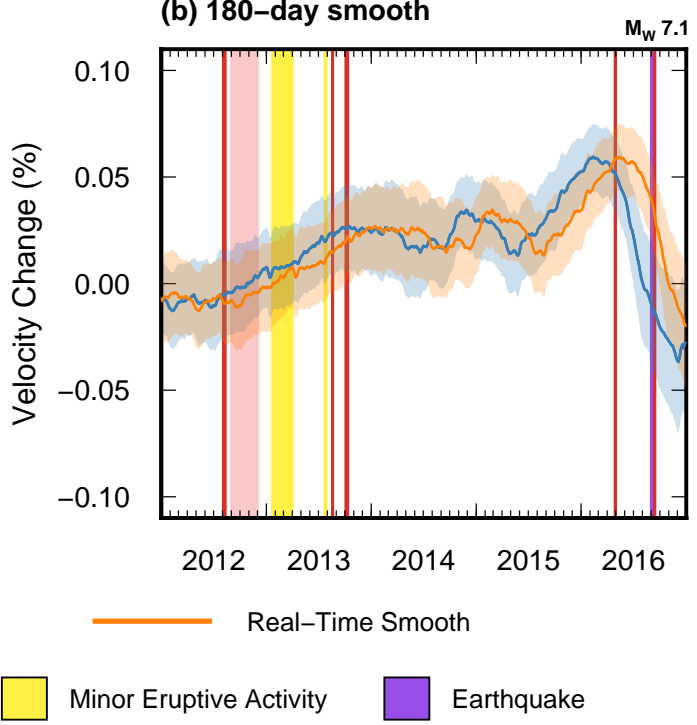

Figure 5.13: Centered moving-window smooth versus a 'real-time' moving-window smooth, demonstrated on $0.1-1.0 \mathrm{~Hz}$ cross-components at WIZ station. The realtime smooth, unlike the centered window, does not use future measurements in the average. (a) 30-day smooth. (b) 180-day smooth. 
to hundreds of kilometers. Many large earthquakes do not however appear to have an immediate effect on volcanoes, which can make the relationship between distant tectonic earthquakes and volcanoes less obvious (Manga and Brodsky, 2006). An interesting result of this thesis was the detection of co-seismic velocity decreases associated with tectonic earthquake activity (Section 4.3.3 and 5.2.2). This is not the first case of earthquake induced velocity changes at volcanoes, with recent work by Lesage et al. (2014) detecting sharp velocity decreases at Volcán de Colima, Mexico, following large tectonic earthquakes. Similarly, large velocity changes were recorded in volcanic regions following the $M_{W} 9.0$ Tohoku-Oki earthquake in Japan (Brenguier et al., 2014). In both cases, changes at volcanoes were related to mechanical weakening of the crust following the passage of seismic waves. Ambient noise monitoring could therefore represent a useful tool for determining the impact of distant tectonic earthquakes at volcanoes. For example, co-seismic velocity decreases were observed following a $M_{W} 7.1$ earthquake but not following a $M_{W} 6.7$ earthquake located at a similar distance away from the volcano (Section 4.3.3). If velocity changes had been monitored in real-time, it could have been determined that crustal changes had occurred following the $M_{W} 7.1$ earthquake. The eruption that occurred two weeks later could potentially then have been anticipated or, at the very least, the probability of an eruption considered to be higher relative to background. At White Island, which poses only a minor risk to human life, this may not have had a significant impact on volcanic hazard preparations. At volcanoes located closer to active populations however, determining whether the volcanic system has been perturbed by distant tectonic earthquakes could be crucial for ensuring civil services, and nearby populations, are prepared for the onset of new activity.

Developing a more complete understanding of background sources of velocity change has been a consistent theme of this discussion. The interaction of volcanic and nonvolcanic processes in recorded seismic velocities makes the interpretation of shortterm precursory activity complicated. Processing onshore stations helped to discriminate between local changes at the volcano and regional changes. For example, it was only with these stations that the $M_{W} 7.1$ earthquake was confidently identified as the source of a velocity decrease in September 2016, instead of a precursor to an eruption two weeks later (Section 4.3.2). However, a more complete understanding of background processes is crucial however to realising the full potential of ambient noise monitoring. 


\section{Chapter 6}

\section{Conclusions}

This thesis relates seismic velocity changes obtained from ambient noise interferometry between 2007-2017 to recent volcanic activity at White Island volcano. Three primary objectives were outlined in Chapter 1, summarised as:

1. Investigate volcanic sources of velocity change and what they tell us about dynamic changes beneath the volcano.

2. Investigate non-volcanic sources of velocity changes and their potential influence on interpretations.

3. Consider the implications for real-time ambient noise monitoring.

Two approaches are applied to monitor the volcano. The station-pair dataset (Chapter 3) used the more common approach of cross-correlating between pairs of seismic stations. Monitoring an island volcano with limited seismic coverage using this approach required noise pathways that included onshore stations. This is potentially problematic, with the spatial resolution of results dramatically reduced. We sought to overcome this by averaging over multiple station-pairs that involved the same seismic station on the volcano. The single-station dataset (Chapter 4), in contrast, recovers velocity changes by cross-correlating the different components of a single seismic station. This represents a less traditional approach to monitoring volcanoes, and may prove to be crucial for environments lacking sufficient seismic coverage. We additionally apply this technique at onshore stations to provide a comparison for interpreting regional, non-volcanic, changes. In this section, we summarise the findings of the thesis (Section 6.1) and recommend potential areas of further work (Section 6.2). 


\subsection{Summary of Findings}

Key findings are outlined here, with Section's 6.1.1, 6.1.2 and 6.1.4 relating directly to the three primary thesis objectives. Section 6.1.3 discusses the suitability of the station-pair and single-station approachs for recording velocity changes at the volcano.

\subsubsection{Volcanic Sources}

Two periods of long-term velocity increase are detected by the single-station dataset that are interpreted to reflect cracks closing under increased pressures beneath the volcano. The first occurs during eruptive activity in 2012-2013 (Fig. 5.11c) and the second in the months preceding the explosive eruption in April 2016 (Fig. 5.11e).

The behaviour of the long-term seismic velocity following moderate explosive eruptions in October 2013 and April 2016 is varied. Following the October 2013 eruptions, the velocity remains elevated relative to levels before the increase a few months earlier (Fig. 5.11d). This could indicate that the pressure source producing the velocity increase has not dissipated as a consequence of the October 2013 eruptions. Following the April 2016 eruption however, the velocity rapidly declines to levels slightly below pre-2015 velocities in the space of 2-3 months (Fig. 5.11f). This is interpreted to reflect a significant reduction in pressure levels following the April 2016 eruption. It is unclear whether the velocity would have continued decreasing to pre-2012 levels without the contribution of the $M_{W} 7.1$ Te Araroa earthquake in September 2016 (see Section 6.1.2).

\subsubsection{Non-Volcanic Sources}

Two tectonic earthquakes are found to have produced significant co-seismic velocity decreases of approximately $0.05-0.1 \%$ (Fig. 4.14). The larger of these - a magnitude $M_{W} 7.1$ in 2016 — was located over $200 \mathrm{~km}$ away from the volcano (Fig. 4.14a). Changes associated with this earthquake are widespread, with decreases detected by single seismic stations both onshore and offshore, and by the station-pair dataset (Fig. 3.18 and 4.14). These changes are therefore interpreted to reflect dynamic stress changes resulting from the passage of seismic waves. The smaller earthquake - a magnitude $M_{W} 5.2$ in 2008 - was located within $10 \mathrm{~km}$ of the volcano (Fig. 4.14e). Strong velocity decreases associated with this event are recorded by a single station on the volcano, with evidence of subsequent post-seismic relaxation in the two years following (Fig. 5.11a). 
This thesis also finds evidence of external sources of seismic velocity changes in the form of seasonal influences. The strongest seasonal trends are recorded by single stations located onshore (Fig. 5.3). We use similarities between annual velocities recorded by these stations and velocities recorded at the volcano to interpret whether long-term changes reflect a volcanic or non-volcanic source process (Fig. 5.12). The information provided by onshore single-station results is crucial to demonstrating that long-term velocity increases in 2012-2013 and 2015-2016 cannot be related to a seasonal trend. This approach could be useful for distinguishing between volcanic and non-volcanic changes at volcanoes globally, in the absence of a more complete understanding of seasonal influences.

\subsubsection{Suitability of Different Approaches}

The majority of interpretable results were provided by the single-station dataset, with minimal evidence to suggest the station-pair dataset was effectively sampling velocity changes at the volcano. The $M_{W} 7.1$ earthquake in 2016 is detected by the station-pair dataset, though changes associated with this earthquake were also observed by onshore single-stations, suggesting a wide regional influence on seismic velocities. This only acts to confirm that the station-pair dataset is correctly sampling crustal velocities, but not the location of these changes. The $M_{W} 5.2$ earthquake, clearly detected in White Island single-station results, is not evident in stationpair results. We therefore consider the single-station dataset more favourable for monitoring Island volcanoes where seismic coverage is sparse. The cross-component correlations in particular are found to produce stable velocity changes, with autocorrelations more prone to temporal changes in the ambient noise wavefield through a lack of spectral whitening in preprocessing.

\subsubsection{Implications for Ambient Noise Monitoring}

Real-time monitoring using ambient noise has potential as an additional tool for forecasting volcanic eruptions. In the short-term, no velocity changes are detected at White Island that could have been clearly identified as indicating an eruption was imminent. This failure to detect short-term pre-cursors could be related to limitations in the temporal resolution of velocity changes, or only minor stress perturbations preceding eruptions relative to background influences. Long-term velocity increases however would have been detectable in real-time (Fig. 5.13) and could have provided valuable information about the behaviour of the volcano alongside other geophysical monitoring tools.

A minor eruption in September 2016 is interpreted here to have been externally trig- 
gered following the $M_{W} 7.1$ earthquake two weeks earlier, with geophysical signals suggesting the volcano was not in a heightened state of unrest. The idea of tectonic triggering of eruptions is not new, yet determining whether a volcanic system has been perturbed by a distant earthquake is less obvious. If real-time monitoring had been in place, it could have been determined that significant crustal changes had occurred following this particular earthquake, with implications for volcanic hazard modelling. At volcanoes located closer to active populations, ambient noise may therefore represent a useful tool for determining whether a volcanic system has been perturbed by distant tectonic earthquakes.

\subsection{Recommendations for Further Work}

The findings of this thesis highlight the promising nature of ambient noise based monitoring and the limitations associated with an incomplete understanding of background processes. We conclude by providing recommendations for further work that support the continued development of ambient noise based volcano monitoring in addition to broader research objectives.

- Analysis of external sources of velocity changes at White Island: A more complete analysis of external sources of velocity change at White Island volcano would improve capabilities to distinguish between volcanic and non-volcanic sources. In this thesis we perform a simplified environmental analysis, comparing meteorological datasets to onshore single-station velocity changes (Section 5.2.1). Meteorological effects are likely to vary however between onshore New Zealand and White Island. A more thorough analysis of seasonal changes local to the volcano is therefore recommended. Additionally, further examples of seismically induced velocity changes may be present within the data, with only earthquakes above magnitude 5 investigated in this thesis. Given strong changes associated with a nearby $M_{W} 5.2$ earthquake, it could be expected that earthquakes below this are capable of similar changes if located close enough to the volcano; for example, volcanic earthquake swarms.

- Investigation of post-2017 velocity changes: At the time of writing mid-2018 - no further volcanic eruptions have occurred at White Island, with activity remaining at low levels. If velocity increases are interpreted to reflect increased pressurization, we might expect that no further increases have occurred between now and the end of 2016 that cannot be related to seasonal changes or post-seismic relaxation following the $M_{W} 7.1$ earthquake. Processing beyond 2016 could therefore explore whether increases in seismic velocity can be used to suggest a heightened state of unrest at the volcano. 
This objective would benefit however from further volcanic activity.

- Further exploration of velocity changes: A significant amount of data has been processed in this thesis, with velocity changes recorded between 20072017 over a large region. Not all of this data is discussed beyond presentation in Sections 3.3 and 4.3, with focus given to only the most reliable interpretations in later discussion. We therefore believe there are many other interesting features to be found within data presented in this thesis, both in a volcanic and non-volcanic context, that we hope can inspire further work using ambient noise. 


\section{Bibliography}

Aki, K. (1957). Space and time spectra of stationary stochastic waves, with special reference to microtremors. Bull. Earth. Res. Inst., 35:415-456.

Aki, K. and Ferrazzini, V. (2000). Seismic monitoring and modeling of an active volcano for prediction. Journal of Geophysical Research, 105(B7):16617-16640.

Anggono, T., Nishimura, T., Sato, H., Ueda, H., and Ukawa, M. (2012). Spatiotemporal changes in seismic velocity associated with the 2000 activity of Miyakejima volcano as inferred from cross-correlation analyses of ambient noise. Journal of Volcanology and Geothermal Research, 247-248:93-107.

Becker, J. S., Saunders, W. S. A., Robertson, C. M., Leonard, G. S., and Johnston, D. M. (2010). A synthesis of challenges and opportunities for reducing volcanic risk through land use planning in New Zealand. Australasian Journal of Disaster and Trauma Studies, 2010(1).

Behr, Y., Townend, J., Bannister, S., and Savage, M. K. (2010). Shear velocity structure of the Northland Peninsula, New Zealand, inferred from ambient noise correlations. Journal of Geophysical Research: Solid Earth, 115(5):1-12.

Belardinelli, M. E., Bizzarri, A., and Cocco, M. (2003). Earthquake triggering by static and dynamic stress changes. Journal of Geophysical Research: Solid Earth, 108(B3):1-16.

Ben-Zion, Y. and Leary, P. (1986). Thermoelastic strain in a half-space covered by unconsolidated material. Bulletin of the Seismological Society of America, $76(5): 1447-1460$.

Bennington, N. L., Haney, M., De Angelis, S., Thurber, C. H., and Freymueller, J. (2015). Monitoring changes in seismic velocity related to an ongoing rapid inflation event at Okmok volcano, Alaska. Journal of Geophysical Research: Solid Earth, 120(8):5664-5676.

Bensen, G. D., Ritzwoller, M. H., Barmin, M. P., Levshin, A. L., Lin, F., Moschetti, M. P., Shapiro, N. M., and Yang, Y. (2007). Processing seismic ambient noise data 
to obtain reliable broad-band surface wave dispersion measurements. Geophysical Journal International, 169(3):1239-1260.

Bensen, G. D., Ritzwoller, M. H., and Shapiro, N. M. (2008). Broadband ambient noise surface wave tomography across the United States. Journal of Geophysical Research: Solid Earth, 113(5):1-21.

Beyreuther, M., Barsch, R., Krischer, L., Megies, T., Behr, Y., and Wassermann, J. (2010). ObsPy: A Python Toolbox for Seismology. Seismological Research Letters, 81(3):530-533.

Black, P. (1970). Observations on White Island Volcano, New Zealand. Bulletin volcanologique, 34(1):158-167.

Bloomberg, S., Werner, C., Rissmann, C., Mazot, A., Horton, T., Graveley, D., Kennedy, B., and Oze, C. (2014). Soil CO2 emissions as a proxy for heat and mass flow assessment, Taupo Volcanic Zone, New Zealand. Geochemistry, Geophysics, Geosystems: G3, 15(12):4885-4904.

Brenguier, F., Campillo, M., Hadziioannou, C., Shapiro, N. M., Nadeau, R. M., and Larose, E. (2008a). Postseismic Relaxation Along the. Science, 321(September):1478-1481.

Brenguier, F., Campillo, M., Takeda, T., Aoki, Y., Shapiro, N. M., Briand, X., Emoto, K., and Miyake, H. (2014). Mapping pressurized volcanic fluids from induced crustal seismic velocity drops. Science, 345(6192):80-82.

Brenguier, F., Clarke, D., Aoki, Y., Shapiro, N. M., Campillo, M., and Ferrazzini, V. (2011). Monitoring volcanoes using seismic noise correlations. Comptes Rendus - Geoscience, 343(8-9):633-638.

Brenguier, F., Shapiro, N. M., Campillo, M., Ferrazzini, V., Duputel, Z., Coutant, O., and Nercessian, A. (2008b). Towards forecasting volcanic eruptions using seismic noise. Nature Geoscience, 1(2):126-130.

Brenguier, F., Shapiro, N. M., Campillo, M., Nercessian, A., and Ferrazzini, V. (2007). 3-D surface wave tomography of the Piton de la Fournaise volcano using seismic noise correlations. Geophysical Research Letters, 34(2):2-6.

Budi-Santoso, A. and Lesage, P. (2016). Velocity variations associated with the large 2010 eruption of Merapi volcano, Java, retrieved from seismic multiplets and ambient noise cross-correlation. Geophysical Journal International, 206(1):221240. 
Campillo, M. and Paul, A. (2003). Long-Range Correlations in the Diffuse Seismic Coda. Science, 299(5606):547-549.

Cayol, V., Dieterich, J. H., Okamura, A. T., and Miklius, A. (2000). High magma storage rates before the 1983 eruption of Kilauea, Hawaii. Science, 288(5475):2343-2346.

Chardot, L. (2015). Geophysical Signature of Unrest Episodes At Active Volcanic Systems: Insights Into the Hydrothermal System Fingerprint. PhD thesis.

Chardot, L., Jolly, A. D., Kennedy, B. M., Fournier, N., and Sherburn, S. (2015). Using volcanic tremor for eruption forecasting at White Island volcano (Whakaari), New Zealand. Journal of Volcanology and Geothermal Research, 302:11-23.

Chen, J. H., Froment, B., Liu, Q. Y., and Campillo, M. (2010). Distribution of seismic wave speed changes associated with the 12 May 2008 Mw 7.9 Wenchuan earthquake. Geophysical Research Letters, 37(18).

Cho, K. H., Herrmann, R. B., Ammon, C. J., and Lee, K. (2007). Imaging the upper crust of the Korean peninsula by surface-wave tomography. Bulletin of the Seismological Society of America, 97(1B):198-207.

Chouet, B. A. (2003). Volcano Seismology. Pure and Applied Geophysics, 160(34):739-788.

Chouet, B. A., Page, R. A., Stephens, C. D., Lahr, J. C., and Power, J. A. (1994). Precursory swarms of long-period events at Redoubt Volcano (1989-1990), Alaska: Their origin and use as a forecasting tool. Journal of Volcanology and Geothermal Research, 62(1-4):95-135.

Christenson, B. W., White, S., Britten, K., and Scott, B. J. (2017). Hydrological evolution and chemical structure of a hyper-acidic spring-lake system on Whakaari/White Island, NZ. Journal of Volcanology and Geothermal Research, 346:180-211.

Claerbout, J. F. (1968). Synthesis of a Layered Medium from its Acoustic Transmission Response. Geophysics, 33(2):264-269.

Clarke, D., Brenguier, F., Froger, J. L., Shapiro, N. M., Peltier, A., and Staudacher, T. (2013). Timing of a large volcanic flank movement at Piton de la Fournaise Volcano using noise-based seismic monitoring and ground deformation measurements. Geophysical Journal International, 195(2):1132-1140. 
Clarke, D., Zaccarelli, L., Shapiro, N. M., and Brenguier, F. (2011). Assessment of resolution and accuracy of the Moving Window Cross Spectral technique for monitoring crustal temporal variations using ambient seismic noise. Geophysical Journal International, 186(2):867-882.

Cole, J. W. and Lewis, K. B. (1981). Evolution of the Taupo-Hikurangi subduction system. Tectonophysics, 72(1-2):1-21.

Cole, J. W., Thordarson, T., and Burt, R. M. (2000). Magma Origin and Evolution of White Island (Whakaari) Volcano, Bay of Plenty, New Zealand. Journal of Petrology, 41(6):867-895.

Colombi, A., Chaput, J., Brenguier, F., Hillers, G., Roux, P., and Campillo, M. (2014). On the temporal stability of the coda of ambient noise correlations. Comptes Rendus - Geoscience, 346(11-12):307-316.

De Plaen, R. S. M., Lecocq, T., Caudron, C., Ferrazzini, V., and Francis, O. (2016). Single station monitoring of volcanoes using seismic ambient noise. Geophysical Research Letters, 43(16):8511-8518.

Donaldson, C., Caudron, C., Green, R. G., Thelen, W. A., and White, R. S. (2017). Relative seismic velocity variations correlate with deformation at $\mathrm{K} \overline{\mathrm{i}}$ lauea volcano. Science Advances, 3:1-12.

Duncan, A. R. (1970). The Petrology and Petrochemistry of Andesite and Dacite Volcanoes in Eastern Bay of Plenty, New Zealand. PhD thesis.

Duputel, Z., Ferrazzini, V., Brenguier, F., Shapiro, N., Campillo, M., and Nercessian, A. (2009). Real time monitoring of relative velocity changes using ambient seismic noise at the Piton de la Fournaise volcano (La Réunion) from January 2006 to June 2007. Journal of Volcanology and Geothermal Research, 184(1-2):164-173.

Duvall, T. L., Jefferies, S. M., Harvey, J. W., and Pomerantz, M. A. (1993). Timedistance Helioseismology. Nature, 362:430-432.

Dzurisin, D. (2003). A comprehensive approach to monitoring volcano deformation as a window on the eruption cycle. Reviews of Geophysics, 41(1):1001.

Eberhart-Phillips, D., Reyners, M., Bannister, S., Chadwick, M., and Ellis, S. (2010). Establishing a Versatile 3-D Seismic Velocity Model for New Zealand. Seismological Research Letters, 81(6):992-1000.

Endo, E. T. and Murray, T. (1991). Real-time Seismic Amplitude Measurement (RSAM): a volcano monitoring and prediction tool. Bulletin of Volcanology, $53(7): 533-545$. 
Fichtner, A., Stehly, L., Ermert, L., and Boehm, C. (2017). Generalized interferometry - I: Theory for interstation correlations. Geophysical Journal International, 208(2):603-638.

Friedrich, A., Krüger, F., and Klinge, K. (1998). Ocean-generated microseismic noise located with the Gräfenberg array. Journal of Seismology, 2(1):47-64.

Froment, B., Campillo, M., Chen, J. H., and Liu, Q. Y. (2013). Deformation at depth associated with the 12 May 2008 MW 7.9 Wenchuan earthquake from seismic ambient noise monitoring. Geophysical Research Letters, 40(1):78-82.

Gerst, A. and Savage, M. K. (2004). Seismic Anisotropy Beneath Ruapehu Volcano: A Possible Eruption Forecasting Tool. Science, 306(November):1543-1547.

Giggenbach, W. F. and Glasby, G. P. (1977). The influence of thermal activity on the trace metal distribution in marine sediments around White Island, New Zealand. New Zealand Department of Science and Industrial Research Bulletin, 218:121-126.

Godfrey, H. J., Fry, B., and Savage, M. K. (2017). Shear-wave velocity structure of the Tongariro Volcanic Centre, New Zealand: Fast Rayleigh and slow Love waves indicate strong shallow anisotropy. Journal of Volcanology and Geothermal Research, 336:33-50.

Gutenberg, B. (1951). Observations and Theory of Microseisms. American Meteorological Society, Boston, MA, pages 1303-1304.

Gutenberg, B. (1958). Microseisms. Advances in Geophysics, 5:53-92.

Hadziioannou, C., Larose, E., Coutant, O., Roux, P., and Campillo, M. (2009). Stability of Monitoring Weak Changes in Multiply Scattering Media with Ambient Noise Correlation: Laboratory Experiments. The Journal of the Acoustical Society of America, 125(6):3688-3695.

Hamling, I. J. (2017). Crater Lake Controls on Volcano Stability: Insights From White Island, New Zealand. Geophysical Research Letters, 44(22):11,311-11,319.

Haney, M. M., Hotovec-Ellis, A. J., Bennington, N. L., De Angelis, S., and Thurber, C. H. (2015). Tracking Changes in Volcanic Systems with Seismic Interferometry. Encyclopedia of Earthquake Engineering, pages 3767-3786.

Hasselmann, K. (1963). A statistical analysis of the generation of microseisms. Reviews of Geophysics, 1(2):177-210. 
Haubrich, R. A. and McCamy, K. (1969). Microseisms: Coastal and pelagic sources. Reviews of Geophysics, 7(3):539-571.

Heckels, R. E. G., Savage, M. K., and Townend, J. (2018). Postseismic velocity changes following the $2010 \mathrm{Mw} 7$. 1 Darfield earthquake, New Zealand, revealed by ambient seismic field analysis. Geophysical Journal International, 213(2):931939.

Herrmann, R. B. (2013). Computer programs in seismology: An evolving tool for instruction and research. Seismological Research Letters, 84(6):1081-1088.

Hillers, G. and Ben-Zion, Y. (2011). Seasonal variations of observed noise amplitudes at 2-18 Hz in southern California. Geophysical Journal International, 184(2):860868.

Hillers, G., Ben-Zion, Y., Campillo, M., and Zigone, D. (2015). Seasonal variations of seismic velocities in the San Jacinto fault area observed with ambient seismic noise. Geophysical Journal International, 202(2):920-932.

Hillers, G., Campillo, M., and Ma, K. F. (2014). Seismic velocity variations at TCDP are controlled by MJO driven precipitation pattern and high fluid discharge properties. Earth and Planetary Science Letters, 391:121-127.

Hiramatsu, Y., Honma, H., Saiga, A., Furumoto, M., and Ooida, T. (2005). Seismological evidence on characteristic time of crack healing in the shallow crust. Geophysical Research Letters, 32(9):1-4.

Hobiger, M., Wegler, U., Shiomi, K., and Nakahara, H. (2012). Coseismic and postseismic elastic wave velocity variations caused by the 2008 Iwate-Miyagi Nairiku earthquake, Japan. Journal of Geophysical Research: Solid Earth, 117(B9).

Hobiger, M., Wegler, U., Shiomi, K., and Nakahara, H. (2014). Single-station crosscorrelation analysis of ambient seismic noise: Application to stations in the surroundings of the 2008 Iwate-Miyagi Nairiku earthquake. Geophysical Journal International, 198(1):90-109.

Houghton, B. F. and Nairn, I. A. (1989). The phreatomagmatic and Strombolian eruption events at White Island volcano 1976-82: eruptive narrative. NZ Geol Surv Bull, 103:13-23.

Houghton, B. F. and Nairn, I. A. (1991). The 1976-1982 Strombolian and phreatomagmatic eruptions of White Island, New Zealand: eruptive and depositional mechanisms at a 'wet' volcano. Bulletin of Volcanology, 54(1):25-49. 
Johnston, D. M., Bebbington, M. S., Chin-Diew, L., Houghton, B. F., and Paton, D. (1999). Volcanic hazard perceptions: comparative shifts in knowledge and risk. Disaster Prevention and Management, 8(2):118-126.

Jolly, A., Kennedy, B., Edwards, M., Jousset, P., and Scheu, B. (2016). Infrasound tremor from bubble burst eruptions in the viscous shallow crater lake of White Island, New Zealand, and its implications for interpreting volcanic source processes. Journal of Volcanology and Geothermal Research, 327:585-603.

Jolly, A. D., Lokmer, I., Thun, J., Salichon, J., Fry, B., and Chardot, L. (2017). Insights into fluid transport mechanisms at White Island from analysis of coupled very long-period (VLP), long-period (LP) and high-frequency (HF) earthquakes. Journal of Volcanology and Geothermal Research, 343:75-94.

King, G. C. P., Stein, R. S., and Lin, J. (1994). Static stress changes and the triggering of earthquakes. Bulletin of the Seismological Society of America, 84(3):935953.

Kobayashi, N. and Nishida, K. (1998). Continuous excitation of planetary free oscillations by atmospheric disturbances. Nature, 395(6700):357-360.

Larose, E., Roux, P., and Campillo, M. (2007). Reconstruction of Rayleigh-Lamb dispersion spectrum based on noise obtained from an air-jet forcing. The Journal of the Acoustical Society of America, 122(6):3437-3444.

Lecocq, T., Caudron, C., and Brenguier, F. (2014). MSNoise, a Python Package for Monitoring Seismic Velocity Changes Using Ambient Seismic Noise. Seismological Research Letters, 85(3):715-726.

Lesage, P., Reyes-Dávila, G., and Arámbula-Mendoza, R. (2014). Large tectonic earthquakes induce sharp temporary decreases in seismic velocity in Volcán de Colima, Mexico. Journal of Geophysical Research : Solid Earth, 119(5):43604376.

Letham-Brake, M. J. (2013). Geological constraints on fluid flow at Whakaari volcano ( White Island). $\mathrm{PhD}$ thesis.

Li, Y. G., Chen, P., Cochran, E. S., Vidale, J. E., and Burdette, T. (2006). Seismic evidence for rock damage and healing on the San Andreas fault associated with the 2004 M 6.0 Parkfield earthquake. Bulletin of the Seismological Society of America, 96(4 B):349-363.

Lin, F.-C., Ritzwoller, M. H., Townend, J., Bannister, S., and Savage, M. K. (2007). Ambient noise Rayleigh wave tomography of New Zealand. Geophysical Journal International, 170(2):649-666. 
Linde, A. T. and Sacks, I. S. (1998). Triggering of volcanic eruptions. Nature, 395(6705):888-890.

Lobkis, O. and Weaver, R. L. (2001). On the emergence of the Green's function in the correlations of a diffuse field. The Journal of the Acoustical Society of America, 110(6):3011-3017.

Lockner, D., Walsh, J., and Byerlee, J. (1977). Changes in seismic velocity and attenuation during deformation of granite. Journal of Geophysical Research, 82(33):5374-5378.

Longuet-Higgins, M. S. (1950). A Theory of the Origin of Microseisms. Philosophical Transactions of the Royal Society A: Mathematical, Physical and Engineering Sciences, 243(857):1-35.

Lu, Z., Mann, D., Freymueller, J. T., and Meyer, D. J. (2000). Synthetic aperture radar interferometry of Okmok volcano, Alaska: Radar observations. Journal of Geophysical Research: Solid Earth, 105(B5):10791-10806.

Manga, M. and Brodsky, E. (2006). Seismic Triggering of Eruptions in the Far Field: Volcanoes and Geysers. Annual Review of Earth and Planetary Sciences, 34(1):263-291.

Marzocchi, W. (2002). Remote seismic influence on large explosive eruptions. Journal of Geophysical Research: Solid Earth, 107(B1):EPM 6-1-EPM 6-7.

McNutt, S. R. (2005). Volcanic Seismology. Annual Review of Earth and Planetary Sciences, 33(1):461-491.

Meier, U., Shapiro, N. M., and Brenguier, F. (2010). Detecting seasonal variations in seismic velocities within Los Angeles basin from correlations of ambient seismic noise. Geophysical Journal International, 181(2):985-996.

Minato, S., Tsuji, T., Ohmi, S., and Matsuoka, T. (2012). Monitoring seismic velocity change caused by the 2011 Tohoku-oki earthquake using ambient noise records. Geophysical Research Letters, 39(9):1-6.

Moon, V., Bradshaw, J., Smith, R., and de Lange, W. (2005). Geotechnical characterisation of stratocone crater wall sequences, White Island Volcano, New Zealand. Engineering Geology, 81(2):146-178.

Mordret, A., Jolly, A. D., Duputel, Z., and Fournier, N. (2010). Monitoring of phreatic eruptions using Interferometry on Retrieved Cross-Correlation Function from Ambient Seismic Noise: Results from Mt. Ruapehu, New Zealand. Journal of Volcanology and Geothermal Research, 191(1-2):46-59. 
Nawa, K., Suda, N., Fukao, Y., and Sato, T. (1998). Incessant excitation of the Earth's free oscillations. Earth, Planets and Space, 50:3-8.

Nishi, Y., Sherburn, S., Scott, B. J., and Sugihara, M. (1996). High-frequency earthquakes at White Island volcano, New Zealand: insights into the shallow structure of a volcano-hydrothermal system. Journal of Volcanology and Geothermal Research, 72(3-4):183-197.

Niu, F., Silver, P. G., Daley, T. M., Cheng, X., and Majer, E. L. (2008). Preseismic velocity changes observed from active source monitoring at the Parkfield SAFOD drill site. Nature, 454(7201):204-208.

Nur, A. (1971). Effects of stress on velocity anisotropy in rocks with cracks. Journal of Geophysical Research, 76(8):2022-2034.

Nur, A. and Simmons, G. (1969). Stress-induced velocity anisotropy in rock: An experimental study. Journal of Geophysical Research, 74(27):6667.

Obermann, A., Planès, T., Larose, E., and Campillo, M. (2013). Imaging preeruptive and coeruptive structural and mechanical changes of a volcano with ambient seismic noise. Journal of Geophysical Research: Solid Earth, 118(12):6285-6294.

Patanè, D., De Gori, P., Chiarabba, C., and Bonaccorso, A. (2003). Magma ascent and the Pressurization of Mount Etna's volcanic system. Science, 299(5615):20612063.

Peltier, A., Scott, B., and Hurst, T. (2009). Ground deformation patterns at White Island volcano (New Zealand) between 1967 and 2008 deduced from levelling data. Journal of Volcanology and Geothermal Research, 181(3-4):207-218.

Peterson, J. (1993). Observations and modeling of seismic background noise.

Poupinet, G., Ellsworth, W. L., and Frechet, J. (1984). Monitoring velocity variations in the crust using earthquake doublets: An application to the Calaveras Fault, California. Journal of Geophysical Research: Solid Earth, 89(B7):57195731.

Prawirodirdjo, L., Ben-Zion, Y., and Bock, Y. (2006). Observation and modeling of thermoelastic strain in Southern California Integrated GPS Network daily position time series. Journal of Geophysical Research: Solid Earth, 111(2):1-10.

Ratdomopurbo, A. and Poupinet, G. (1995). Monitoring a temporal change of seismic velocity in a volcano: Application to the 1992 eruption of Mt. Merapi (Indonesia). Geophysical Research Letters, 22(7):775-778. 
Rhie, J. and Romanowicz, B. (2004). Excitation of Earth 's continuous free oscillations by atmosphere - ocean - seafloor coupling. Nature, 431(September):552-556.

Richter, T., Sens-schönfelder, C., Kind, R., and Asch, G. (2014). Journal of Geophysical Research : Solid Earth Comprehensive observation and modeling of earthquake and temperature-related seismic velocity changes in northern Chile with passive image interferometry Journal of Geophysical Research : Solid Earth. Journal of Geophysical Research, 119(6):4747-4765.

Rivet, D., Brenguier, F., and Cappa, F. (2015). Improved detection of preeruptive seismic velocity drops at the Piton de La Fournaise volcano. Geophysical Research Letters, 42(15):6332-6339.

Rivet, D., Brenguier, F., Clarke, D., Shapiro, N. M., and Peltier, A. (2014). Longterm dynamics of Piton de la Fournaise volcano from 13 years of seismic velocity change measurements and GPS observations. Journal of Geophysical Research: Solid Earth, 119:7654-7666.

Sabra, K. G., Gerstoft, P., Roux, P., Kuperman, W. A., and Fehler, M. C. (2005). Extracting time-domain Green's function estimates from ambient seismic noise. Geophysical Research Letters, 32(3):1-5.

Sánchez-Sesma, F. J. and Campillo, M. (2006). Retrieval of the Green's function from cross correlation: The canonical elastic problem. Bulletin of the Seismological Society of America, 96(3):1182-1191.

Sato, H., Fehler, M. C., and Maeda, T. (2012). Seismic Wave Propagation and Scattering in Heterogeneous Earth. Berlin: Springer, 496.

Savage, M. K., Ferrazzini, V., Peltier, A., Rivemale, E., Mayor, J., Schmid, A., Brenguier, F., Massin, F., Got, J., Battaglia, J., Dimuro, A., Staudacher, T., Rivet, D., Taisne, B., and Shelley, A. (2015). Seismic anisotropy and its precursory change before eruptions at Piton de la Fournaise volcano, Le Réunion. Journal of Geophysical Research: Solid Earth, 120(5):3430-3458.

Schaff, D. P. and Beroza, G. C. (2004). Coseismic and postseismic velocity changes measured by repeating earthquakes. Journal of Geophysical Research: Solid Earth, $109(10)$.

Schimmel, M. and Paulssen, H. (1997). Noise reduction and detection of weak, coherent signals through phase weighted stacks. Geophysical Journal International, 130:497-505.

Sens-Schönfelder, C. (2008). Synchronizing seismic networks with ambient noise. Geophysical Journal International, 174(3):966-970. 
Sens-Schönfelder, C. and Wegler, U. (2006). Passive image interferemetry and seasonal variations of seismic velocities at Merapi Volcano, Indonesia. Geophysical Research Letters, 33(21):1-5.

Shapiro, N. M. and Campillo, M. (2004). Emergence of broadband Rayleigh waves from correlations of the ambient seismic noise. Geophysical Research Letters, $31(7): 8-11$.

Shapiro, N. M., Campillo, M., Stehly, L., and Ritzwoller, M. H. (2005). HighResolution Surface-Wave Tomography from Ambient Seismic Noise. Science, 307(5715):1615-1618.

Sherburn, S., Scott, B. J., Nishi, Y., and Sugihara, M. (1998). Seismicity at White Island volcano, New Zealand: a revised classification and inferences about source mechanism. Journal of Volcanology and Geothermal Research, 83(3-4):287-312.

Silver, P. G., Daley, T. M., Niu, F., and Majer, E. L. (2007). Active source monitoring of cross-well seismic travel time for stress-induced changes. Bulletin of the Seismological Society of America, 97(1B):281-293.

Snieder, R. (2004). Extracting the Green's function from the correlation of coda waves: A derivation based on stationary phase. Physical Review E - Statistical Physics, Plasmas, Fluids, and Related Interdisciplinary Topics, 69(4):046610.

Snieder, R., Grêt, A., Douma, H., and Scales, J. (2002). Coda Wave Interferometry for in Seismic Velocity. Science, 295(5563):2253-2255.

Sparks, R. S. (2003). Forecasting volcanic eruptions. Earth and Planetary Science Letters, 210(1-2):1-15.

Stein, S. and Wysession, M. (2003). An Introduction to Seismology, Earthquakes, and Eart Structure.

Stern, T. A. (1987). Asymmetric back-arc spreading, heat flux and structure associated with the Central Volcanic Region of New Zealand. Earth and Planetary Science Letters, 85(1-3):265-276.

Stutzmann, E., Roult, G., and Astiz, L. (2000). GEOSCOPE station noise levels. Bulletin of the Seismological Society of America, 90(3):690-701.

Suda, N., Nawa, K., and Fukao, Y. (1998). Earth's Background Free Oscillations. Science, 279(5359):2089-2091.

Taira, T., Brenguier, F., and Kong, Q. (2015). Ambient Noise Based Monitoring of Seismic Velocity Changes Associated with the 2014 Mw 6 . 0 South Napa Earthquake. (September 2010):1-8. 
Takagi, R., Okada, T., Nakahara, H., Umino, N., and Hasegawa, A. (2012). Coseismic velocity change in and around the focal region of the 2008 Iwate-Miyagi Nairiku earthquake. Journal of Geophysical Research: Solid Earth, 117(6):1-19.

Takano, T., Nishimura, T., Nakahara, H., Ohta, Y., and Tanaka, S. (2014). Seismic velocity changes caused by the Earth tide: Ambient noise correlation analyses of a small array data. Geophysical Research Letters, 41(17):6131-6136.

Tsai, V. C. (2010). The relationship between noise correlation and the Green's function in the presence of degeneracy and the absence of equipartition. Geophysical Journal International, 182(3):1509-1514.

Tsai, V. C. (2011). A model for seasonal changes in GPS positions and seismic wave speeds due to thermoelastic and hydrologic variations. Journal of Geophysical Research: Solid Earth, 116(4):1-9.

Wang, Q. Y., Brenguier, F., Campillo, M., Lecointre, A., Takeda, T., and Aoki, Y. (2017). Seasonal Crustal Seismic Velocity Changes Throughout Japan. Journal of Geophysical Research: Solid Earth, 122(10):7987-8002.

Wapenaar, K. (2006). Green's function retrieval by cross-correlation in case of onesided illumination. Geophysical Research Letters, 33(19):1-6.

Wapenaar, K., Draganov, D., Snieder, R., Campman, X., and Verdel, A. (2010). Tutorial on seismic interferometry: Part 1 - Basic principles and applications. Geophysics, 75(5):75A195-75A209.

Warren-Smith, E., Fry, B., Kaneko, Y., and Chamberlain, C. J. (2018). Foreshocks and delayed triggering of the 2016 MW7.1 Te Araroa earthquake and dynamic reinvigoration of its aftershock sequence by the MW7.8 Kaikōura earthquake, New Zealand. Earth and Planetary Science Letters, 482:265-276.

Weaver, R. L. and Lobkis, O. I. (2001). Ultrasonics without a source: Thermal fluctuation correlations at mhz frequencies. Physical Review Letters, 87(13):1-4.

Wegler, U. and Lühr, B. G. (2001). Scattering behaviour at Merapi volcano (Java) revealed from an active seismic experiment. Geophysical Journal International, 145(3):579-592.

Wegler, U., Lühr, B. G., Snieder, R., and Ratdomopurbo, A. (2006). Increase of shear wave velocity before the 1998 eruption of Merapi volcano (Indonesia). Geophysical Research Letters, 33(9):4-7.

Wegler, U. and Sens-Schönfelder, C. (2007). Fault zone monitoring with passive image interferometry. Geophysical Journal International, 168(3):1029-1033. 
Werner, C., Hurst, T., Scott, B., Sherburn, S., Christenson, B. W., Britten, K., Cole-

Baker, J., and Mullan, B. (2008). Variability of passive gas emissions, seismicity, and deformation during crater lake growth at White Island Volcano, New Zealand, 2002-2006. Journal of Geophysical Research: Solid Earth, 113(B1).

Wright, I. C. (1992). Shallow structure and active tectonism of an offshore continental back-arc spreading system: the Taupo Volcanic Zone, New Zealand. Marine Geology, 103(1-3):287-309.

Yamamura, K., Sano, O., Utada, H., Takei, Y., Nakao, S., and Fukao, Y. (2003). Long-term observation of in situ seismic velocity and attenuation. Journal of Geophysical Research: Solid Earth, 108(B6).

Yang, Y., Ritzwoller, M. H., Levshin, A. L., and Shapiro, N. M. (2007). Ambient noise Rayleigh wave tomography across Europe. Geophysical Journal International, 168(1):259-274.

Yao, H., Beghein, C., and Van Der Hilst, R. D. (2006). Surface wave array tomography in SE Tibet from ambient seismic noise and two-station analysis - II. Crustal and upper-mantle structure. Geophysical Journal International, 166(2):732-744.

Young, C. J., Chael, E. P., Zagar, D. A., and Carter, J. A. (1994). Variations in Noise and Signal Levels in a Pair of Deep Boreholes near Amarillo, Texas. Bulletin of the Seismological Society of America, 84(5):1593-1607. 


\section{Appendix A}

\section{Data Processing Supplementary Material}

This Appendix Chapter contains supplementary information relating to processing. This includes information about seismic stations used in this study (Section A.1) and the final MSNoise parameters chosen for both single-station and station-pair datasets (Section A.2). Also included is information about the timing correction applied to the station-pair dataset (Section A.3), supplementary figures showing improvements made after increasing the correlation length (Section A.4) in the single-station dataset, and the velocity model used to compute depth sensitivity kernels (Section A.5). 


\section{A.1 Seismic Station Details}

Table A.1: Seismic Station Information for both datasets used in this thesis. Multiple rows are used if a station sensor has an end date between 2007-2017

\begin{tabular}{|c|c|c|c|c|}
\hline Station Name & Make & Model & Start Date & End Date \\
\hline HAZ & Guralp Systems Ltd. & CMG-3ESPC & $2010-01-14$ & N/A \\
\hline KUZ & Guralp Systems Ltd. & CMG-3ESP & $2004-02-05$ & N/A \\
\hline MWZ & Guralp Systems Ltd. & CMG-3ESP & $2004-02-29$ & N/A \\
\hline MXZ & Guralp Systems Ltd. & CMG-3ESP & $2004-02-29$ & $2009-08-17$ \\
\hline MXZ & Guralp Systems Ltd. & CMG-3ESPC & $2009-08-17$ & N/A \\
\hline OPRZ & Guralp Systems Ltd. & CMG-3ESP & $2007-06-19$ & $2008-11-20$ \\
\hline OPRZ & Guralp Systems Ltd. & CMG-3ESP & $2008-11-20$ & N/A \\
\hline PUZ & Guralp Systems Ltd. & CMG-3ESP & $2003-08-20$ & N/A \\
\hline TOZ & Guralp Systems Ltd. & CMG-40T-30S & $1998-10-15$ & $2008-12-04$ \\
\hline TOZ & Guralp Systems Ltd. & CMG-3ESPC & $2008-12-04$ & N/A \\
\hline URZ & Guralp Systems Ltd. & CMG-3TB & $2002-02-19$ & $2016-09-13$ \\
\hline URZ & Guralp Systems Ltd. & CMG-3TB & $2016-09-14$ & N/A \\
\hline WIZ & Sercel Inc. & L4C & $1993-08-24$ & $2007-04-19$ \\
\hline WIZ & Guralp Systems Ltd. & CMG-3ESP & $2007-04-19$ & 2016-09-13 \\
\hline WIZ & Nanometrics Inc. & Trillium 120QA & $2016-09-13$ & N/A \\
\hline WSRZ & Guralp Systems Ltd. & CMG-3ESPC & $2013-04-08$ & N/A \\
\hline
\end{tabular}




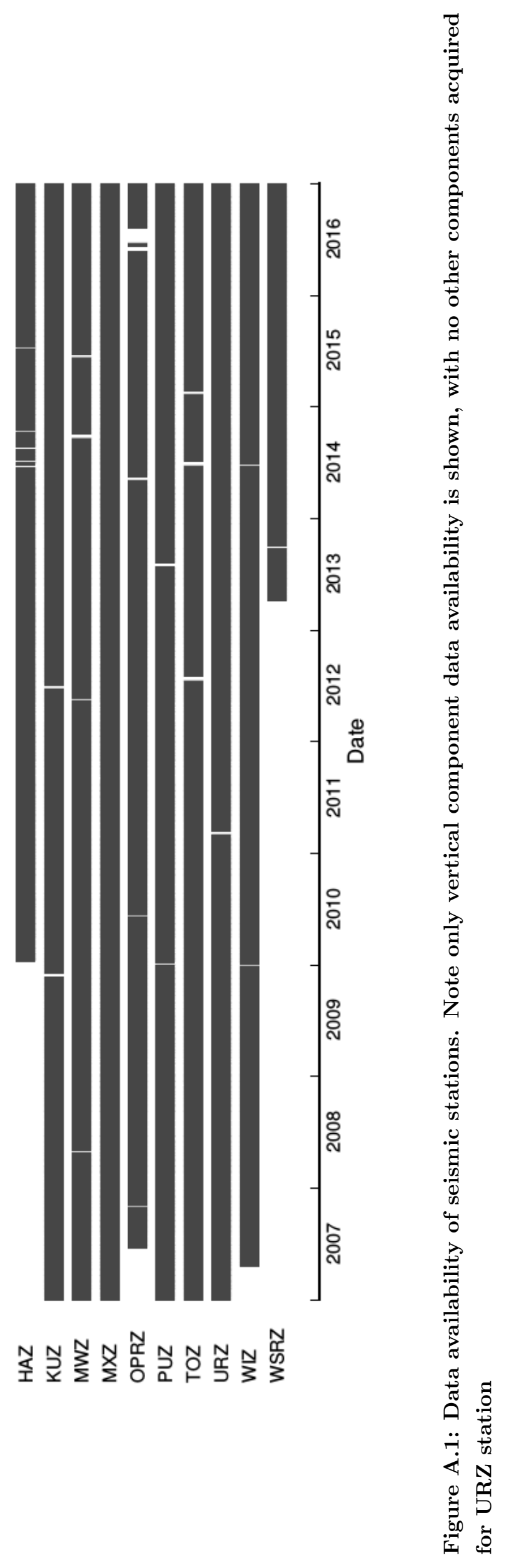




\section{A.2 Final MSNoise Parameters}

This section contains the parameter choices used within MSNoise to process raw ambient noise data through to velocity details. Parameters for the station-pair dataset are found in Section A.2.1 and parameters for the station-pair dataset found in Section A.2.2. MSNoise version 1.4 is used to process the station-pair dataset and version 1.5.1 used to process the single-station dataset. This decision relates only to version 1.5 becoming available midway through the project, allowing single-station processing within MSNoise.

\section{A.2.1 Station-pair Dataset}

Table A.2: Station-pair dataset Preprocessing Parameters (MSNoise Version 1.4). Where value is ' $N / A$ ', the parameter was part of customised scripts, with information provided in Table A.4

\begin{tabular}{|c|l|c|}
\hline Parameter & Description & Value \\
\hline analysis_duration & Duration of analysis & 86400 seconds \\
\hline autocorr & Compute auto-correlations & No \\
\hline cc_sampling_rate & Sampling rate for CCFs & Hz \\
\hline channels & Seismic channels & HHE, HHN, HHZ \\
\hline corr_duration & Length of data windows to correlate & 3600 seconds \\
\hline dtt_lag & How the lag time is defined (dynamic or static) & N/A (p135) \\
\hline dtt_maxdt & Maximum delay time measurement & N/A (p135) \\
\hline dtt_maxerr & Minimum error on delay time measurement & N/A (p135) \\
\hline dtt_mincoh & Minimum coherence on delay time measurement & 0.85 \\
\hline dtt_minlag & Minimum lag for delay time measurements & N/A (p135) \\
\hline dtt_sides & Which sides of CCFs to use for delay times & both \\
\hline dtt_width & Width of time lag window & N/A (p135) \\
\hline enddate & End date of data & $2017-01-01$ \\
\hline maxlag & Maximum lag of CCFs & 500 seconds \\
\hline mov_stack & Stack size & 30 days \\
\hline preprocess_highpass & Preprocessing high-pass value & $0.01 \mathrm{~Hz}$ \\
\hline preprocess_lowpass & Preprocessing low-pass value & $0.4 \mathrm{~Hz}$ \\
\hline ref_begin & Reference stack start date & $2007-01-01$ \\
\hline ref_end & Reference stack end date & $2017-01-01$ \\
\hline resampling_method & Resampling method (resample / decimate / lanczos) & Resample \\
\hline stack_method & Stack Method: Linear mean or phase weighted stack & linear \\
\hline startdate & Start date of data & $2007-01-01$ \\
\hline windsorizing & Windorizing at N times RMS & 3 \\
\hline
\end{tabular}


Table A.3: Station-pair dataset filter table (MSNoise Version 1.4)

\begin{tabular}{|c|l|c|}
\hline Parameter & Description & Value \\
\hline Low & The lower frequency bound of the whiten function & $0.03 \mathrm{~Hz}$ \\
\hline Mwcs low & The lower frequency bound of the linear regression done in MWCS & $0.04 \mathrm{~Hz}$ \\
\hline Mwcs high & The upper frequency bound of the linear regression done in MWCS & $0.34 \mathrm{~Hz}$ \\
\hline High & The upper frequency bound of the whiten function & $0.35 \mathrm{~Hz}$ \\
\hline Mwcs wlen & Window length to perform MWCS & 20 seconds \\
\hline Mwcs step & Step of the windowing procedure in MWCS & 4 seconds \\
\hline
\end{tabular}

Table A.4: Customised parameters used for measuring velocity changes from delay times in station-pair dataset. Minimum $(\min )$ and maximum $(\max )$ lag times are defined for velocity analysis at for both positive (+ve) and negative (-ve) lag times. Maximum delay times and errors are also included ( $\mathrm{dt}$ and error respectively).

\begin{tabular}{|c|c|c|c|c|c|c|}
\hline Station-pair & Max lag (-ve) & Min lag (-ve) & Max lag (+ve) & Max lag (+ve) & Max dt & Max error \\
\hline HAZ - WIZ / WSRZ & 40 & 45 & 105 & 120 & 0.6 & 0.1 \\
\hline MWZ - WIZ / WSRZ & 55 & 60 & 130 & 140 & 0.7 & 0.1 \\
\hline OPRZ - WIZ / WSRZ & 55 & 55 & 145 & 135 & 0.7 & 0.1 \\
\hline PUZ - WIZ / WSRZ & 65 & 65 & 140 & 135 & 0.7 & 0.1 \\
\hline URZ - WIZ / WSRZ & 55 & 55 & 150 & 115 & 0.7 & 0.1 \\
\hline
\end{tabular}




\section{A.2.2 Single-Station Dataset}

Table A.5: Preprocessing Parameters for Single-Station Dataset (MSNoise Version 1.5.1)

\begin{tabular}{|c|c|c|c|}
\hline Parameter & Description & Dataset & Value \\
\hline analysis_duration & Duration of analysis & $\mathrm{N} / \mathrm{A}$ & 86400 seconds \\
\hline autocorr & Compute auto-correlations & $\mathrm{N} / \mathrm{A}$ & Y \\
\hline cc_sampling_rate & Sampling rate for CCFs & $\mathrm{N} / \mathrm{A}$ & $10 \mathrm{~Hz}$ \\
\hline channels & Seismic channels & $\mathrm{N} / \mathrm{A}$ & HHE, HHN, HHZ \\
\hline corr_duration* & Length of data windows to correlate & $0.1-1.0 \mathrm{~Hz}$ & 7200 seconds \\
\hline corr_duration* & Length of data windows to correlate & $1.0-2.0 \mathrm{~Hz}$ & 14400 seconds \\
\hline dtt_lag & How the lag time is defined (dynamic or static) & $\mathrm{N} / \mathrm{A}$ & static \\
\hline dtt_maxdt* & Maximum delay time measurement & $0.1-1.0 \mathrm{~Hz}$ & 0.4 seconds \\
\hline dtt_maxdt* & Maximum delay time measurement & $1.0-2.0 \mathrm{~Hz}$ & 0.2 seconds \\
\hline dtt_maxerr & Minimum error on delay time measurement & $\mathrm{N} / \mathrm{A}$ & 0.1 seconds \\
\hline dtt_mincoh* & Minimum coherence on delay time measurement & $0.1-1.0 \mathrm{~Hz}$ & 0.8 \\
\hline dtt_mincoh* & Minimum coherence on delay time measurement & $1.0-2.0 \mathrm{~Hz}$ & 0.6 \\
\hline dtt_minlag* & Minimum lag for delay time measurements & $0.1-1.0 \mathrm{~Hz}$ & 20 seconds \\
\hline dtt_minlag* & Minimum lag for delay time measurements & $1.0-2.0 \mathrm{~Hz}$ & 10 seconds \\
\hline dtt_sides & Which sides of CCFs to use for delay times & $\mathrm{N} / \mathrm{A}$ & both \\
\hline dtt_width* & Width of time lag window & $0.1-1.0 \mathrm{~Hz}$ & 60 seconds \\
\hline dtt_width* & Width of time lag window & $1.0-2.0 \mathrm{~Hz}$ & 30 seconds \\
\hline enddate & End date of data & $\mathrm{N} / \mathrm{A}$ & 2017-01-01 \\
\hline maxlag & Maximum lag of CCFs & $\mathrm{N} / \mathrm{A}$ & 120 seconds \\
\hline mov_stack & Stack size & $\mathrm{N} / \mathrm{A}$ & 10 days \\
\hline preprocess_highpass* & Preprocessing high-pass value & $0.1-1.0 \mathrm{~Hz}$ & $0.1 \mathrm{~Hz}$ \\
\hline preprocess_lowpass* & Preprocessing low-pass value & $0.1-1.0 \mathrm{~Hz}$ & $1.0 \mathrm{~Hz}$ \\
\hline preprocess_highpass* & Preprocessing high-pass value & $1.0-2.0 \mathrm{~Hz}$ & $1.0 \mathrm{~Hz}$ \\
\hline preprocess_lowpass* & Preprocessing low-pass value & $1.0-2.0 \mathrm{~Hz}$ & $2.0 \mathrm{~Hz}$ \\
\hline ref_begin & Reference stack start date & $\mathrm{N} / \mathrm{A}$ & 2007-01-01 \\
\hline ref_end & Reference stack end date & $\mathrm{N} / \mathrm{A}$ & 2017-01-01 \\
\hline resampling_method & Resampling method (resample / decimate / lanczos) & $\mathrm{N} / \mathrm{A}$ & Decimate \\
\hline stack_method & Stack Method: Linear mean or phase weighted stack & $\mathrm{N} / \mathrm{A}$ & linear \\
\hline startdate & Start date of data & $\mathrm{N} / \mathrm{A}$ & $2007-01-01$ \\
\hline whitening & Whiten traces $(\mathrm{A}=$ all except autocorrelations $)$ & $\mathrm{N} / \mathrm{A}$ & A \\
\hline windsorizing & Windsorizing at $\mathrm{N}$ times RMS & $\mathrm{N} / \mathrm{A}$ & 3 \\
\hline
\end{tabular}

Table A.6: White Island dataset 0.1-1.0 Hz Filter Table (MSNoise Version 1.5.1)

\begin{tabular}{|c|l|c|}
\hline Parameter & Description & Value \\
\hline Low & The lower frequency bound of the whiten function & $0.12 \mathrm{~Hz}$ \\
\hline Mwcs low & The lower frequency bound of the linear regression done in MWCS & $0.14 \mathrm{~Hz}$ \\
\hline Mwcs high & The upper frequency bound of the linear regression done in MWCS & $0.96 \mathrm{~Hz}$ \\
\hline High & The upper frequency bound of the whiten function & $0.98 \mathrm{~Hz}$ \\
\hline Mwcs wlen & Window length to perform MWCS & 16 seconds \\
\hline Mwcs step & Step of the windowing procedure in MWCS & 4 seconds \\
\hline
\end{tabular}


Table A.7: White Island dataset 1.0-2.0 Hz Filter Table (MSNoise Version 1.5.1)

\begin{tabular}{|c|l|c|}
\hline Parameter & Description & Value \\
\hline Low & The lower frequency bound of the whiten function & $1.02 \mathrm{~Hz}$ \\
\hline Mwcs low & The lower frequency bound of the linear regression done in MWCS & $1.04 \mathrm{~Hz}$ \\
\hline Mwcs high & The upper frequency bound of the linear regression done in MWCS & $1.96 \mathrm{~Hz}$ \\
\hline High & The upper frequency bound of the whiten function & $1.98 \mathrm{~Hz}$ \\
\hline Mwcs wlen & Window length to perform MWCS & 12 seconds \\
\hline Mwcs step & Step of the windowing procedure in MWCS & 4 seconds \\
\hline
\end{tabular}




\section{A.3 Time Shift Correction (Station-Pairs)}

Timing errors in seismic records can result in shifted cross-correlation functions such that are no longer symmetric about zero lag, ignoring asymmetry resulting from non-homogeneous distributions of noise sources. These shifts can produce unreliable measurements during the moving-window cross-spectral technique stage, with delay times not following a systematic pattern that would be associated with changes in velocity. Correcting for time shifts is found to be particularly important in this thesis, with a significant time shift observed in cross-correlation functions involving WIZ station from mid-2014 (Fig. A.2).

A correction is applied for time shifts in two stages. First, for each station-pair involving WIZ, 1-day cross-correlation functions are shifted by 0.6 seconds from 7 July 2014. Then, the time lag is measured between these 1-day cross-correlation functions and the reference function, following the method of Sens-Schönfelder (2008). If the measured time lag is over a threshold value, chosen here to be 0.45 seconds, the waveform is shifted by the recorded lag in an attempt to reduce the time shift to zero. The reference stack is then recomputed and time lags. Further iteration is optional but not applied here. Figure A.3 shows the final time shifts between shifted
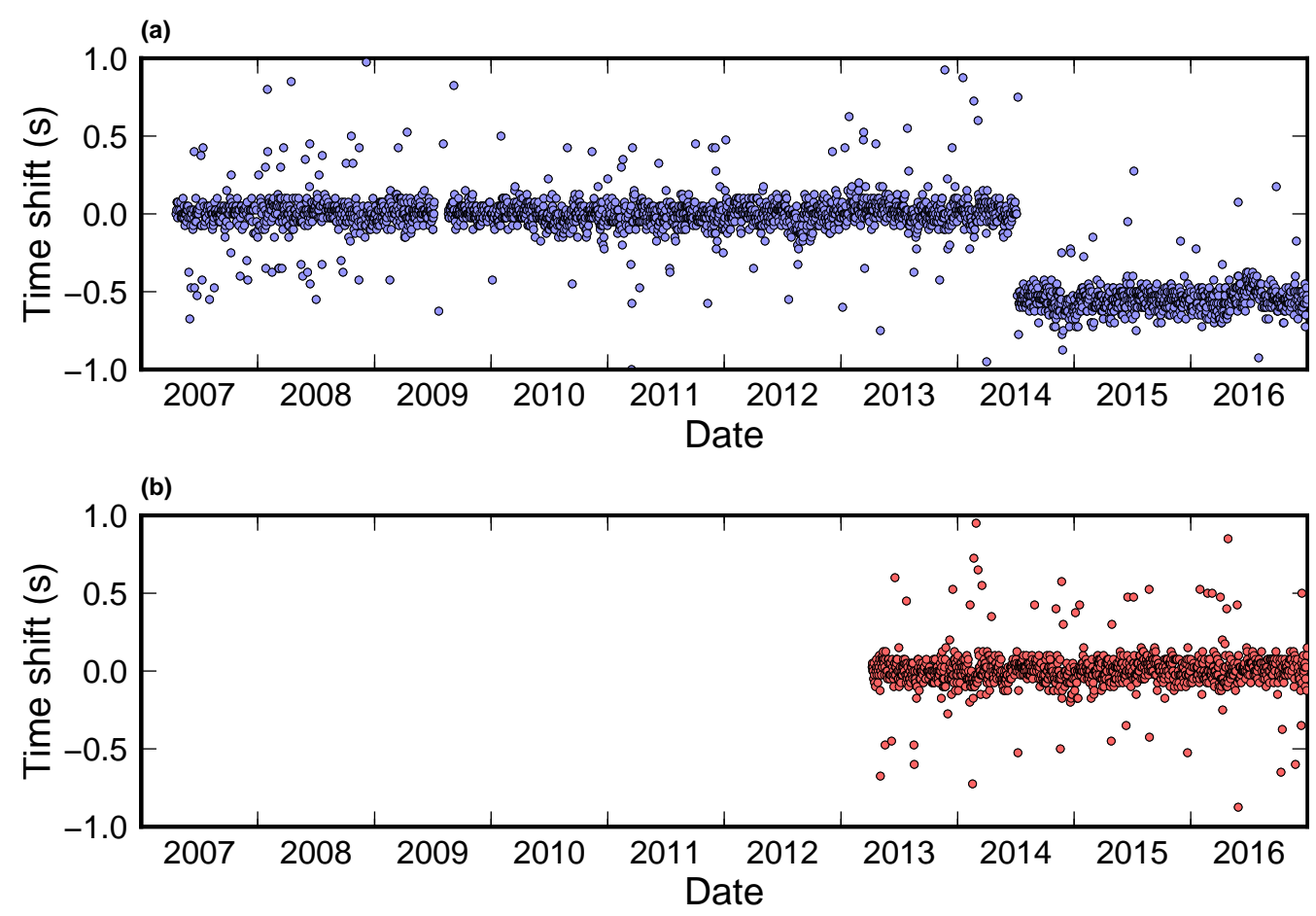

Figure A.2: Average time shift between single day and reference CCFs for (a): all station-pairs including station WIZ, with a reference stack from 1 January 2007 to 1 January 2012, (b): all station-pairs including station WSRZ, with a reference stack from 1 April 2013 to 1 January 2017. 

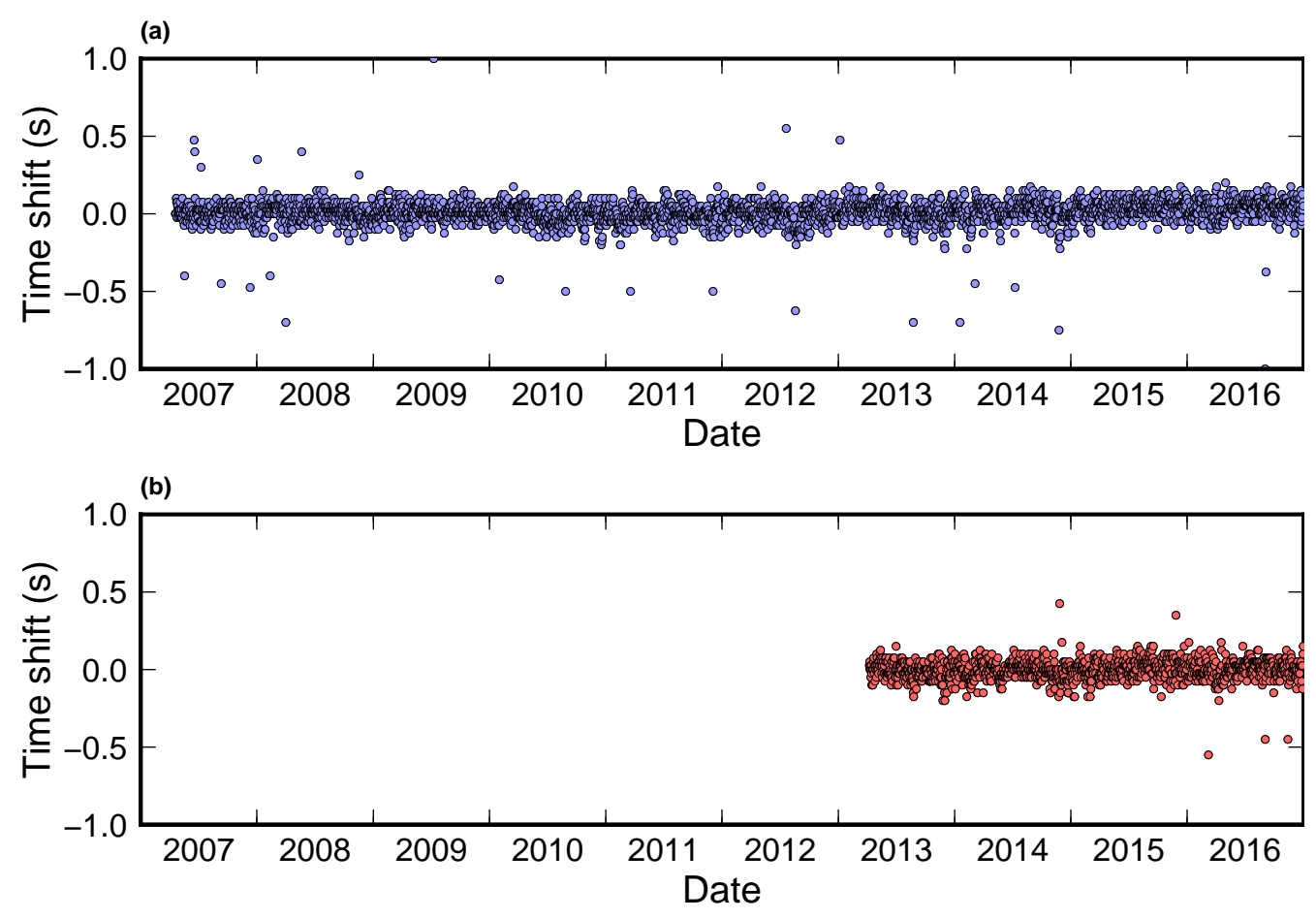

Figure A.3: Average shift between single day and reference CCFs after one iteration of timing error correction. (a): all station-pairs including station WIZ, with a reference stack from 1 January 2007 to 1 January 2012, (b): all station-pairs including station WSRZ, with a reference stack from 1 April 2013 to 1 January 2017.

1-day cross-correlation functions and the recomputed reference stack. Note that the step is no longer visible mid-2014. Remaining time-shifts that fall outside of the defined threshold are assumed to be related to days where SNR is low, rendering time shift measurements unreliable. 


\section{A.4 Correlation Length Influence on Single-Station Processing}
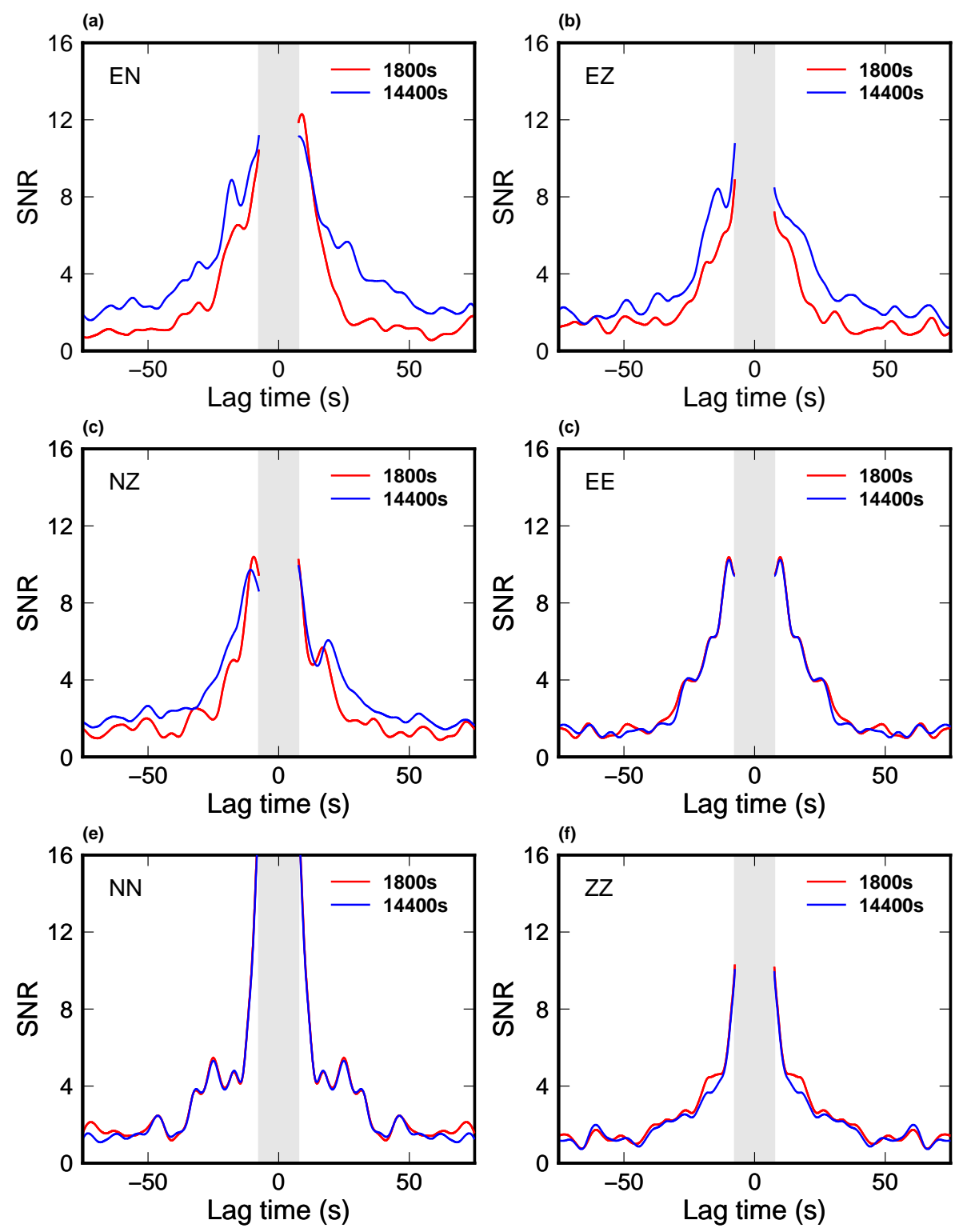

Figure A.4: Influence of increasing the correlation length on the SNR of $1.0-2.0 \mathrm{~Hz}$ cross-correlation functions, shown individually for different component-pairs. Correlation lengths of 1800 seconds and 14400 seconds are shown. 
(a) Corr. length $=1800 \mathrm{~s}$

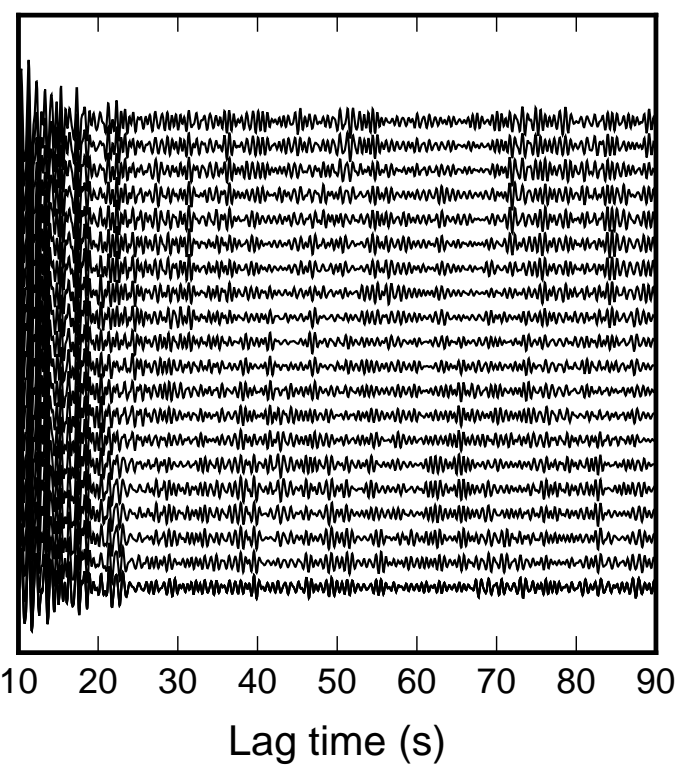

(b) Corr. length $=14400 \mathrm{~s}$

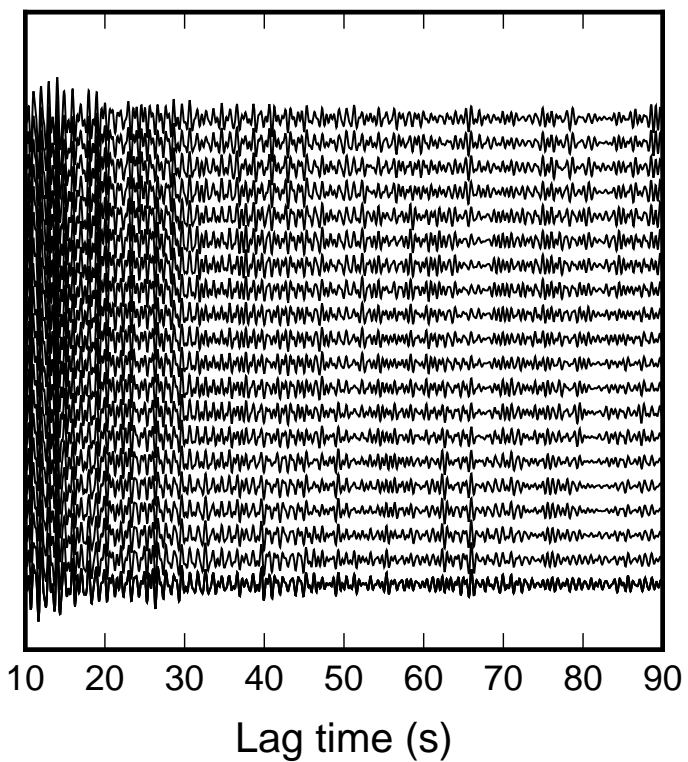

Figure A.5: Ten-day 1.0-2.0 Hz stacks, recorded by component-pair EN, are plotted for 20 consecutive days after applying two different correlation lengths. (a) 1800 seconds. (b) 14400 seconds. 


\section{A.5 Velocity Model for Sensitivity Kernel}

The velocity model used to compute depth sensitivity kernels is acquired from the supplementary material of Eberhart-Phillips et al. (2010), who constructed a 3-D seismic velocity model of New Zealand using local earthquake data. We use a 2-D velocity profile from this model, choosing the profile that is closest geographically to White Island. The chosen profile has latitude of -37.5698 and longitude of 177.1315, with $x$ and $y$ coordinates of $(x, y)=(-8,-590)$ in the model. Compressional and shear wave velocities corresponding to this model, in addition to densities, are shown in Figure A.6.
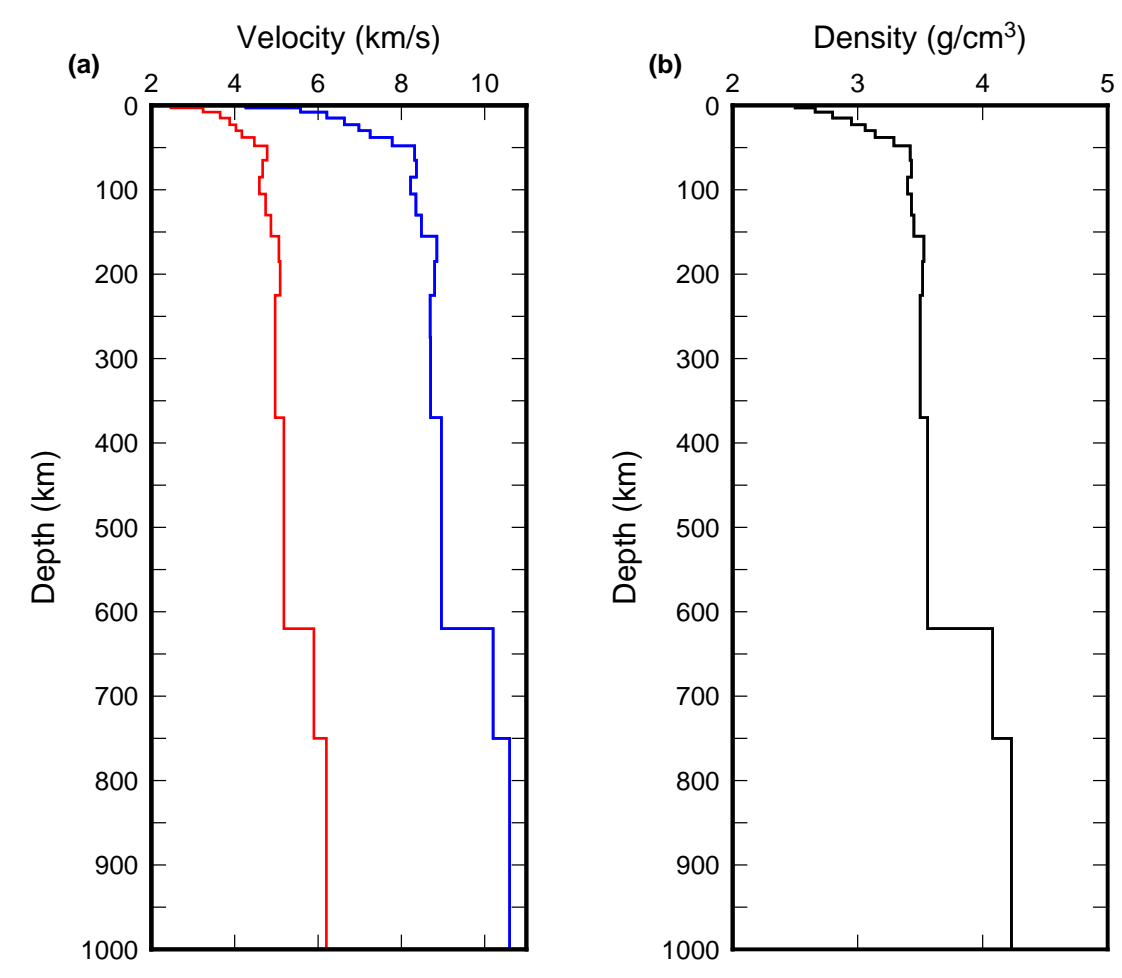

Figure A.6: Velocity model parameters acquired from (Eberhart-Phillips et al., 2010), using the 2D profile corresponding to (latidude, longitude $)=(-37.5698,177.1315)$. (a) Compressional (blue line) and shear wave velocity (red line) profiles. (b) Density Profile. 


\section{Appendix B}

\section{Velocity Changes Supplementary Material}

This Appendix chapter contains figures of velocity changes excluded from the main portion of this thesis. This includes velocity changes computed for individual component-pairs of the single-station dataset (Section B.1), station-pair WIZ-WSRZ (Section B.2) and OPRZ single-station results (Section B.3). 


\section{B.1 Individual single-station component-pairs}

(a)

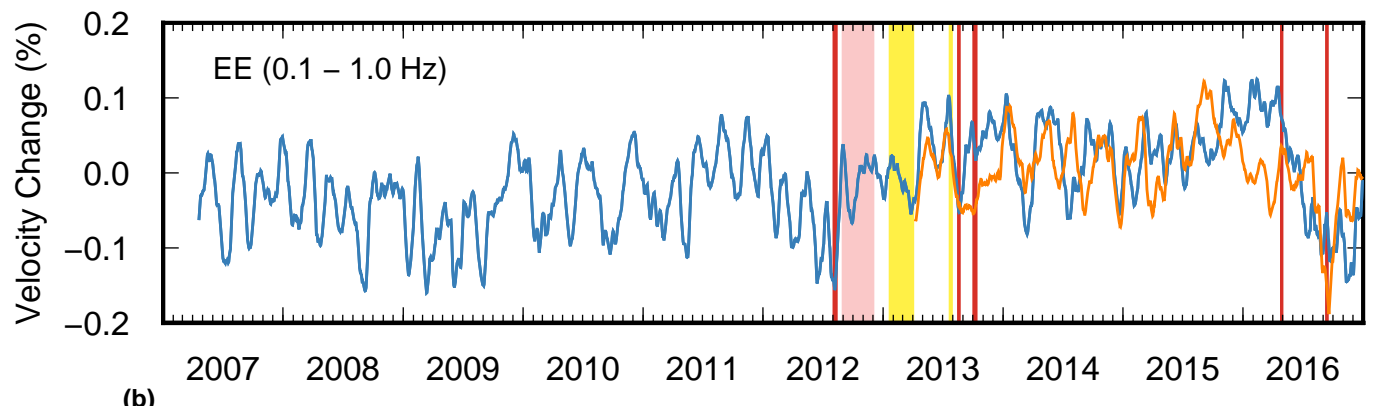

(b)

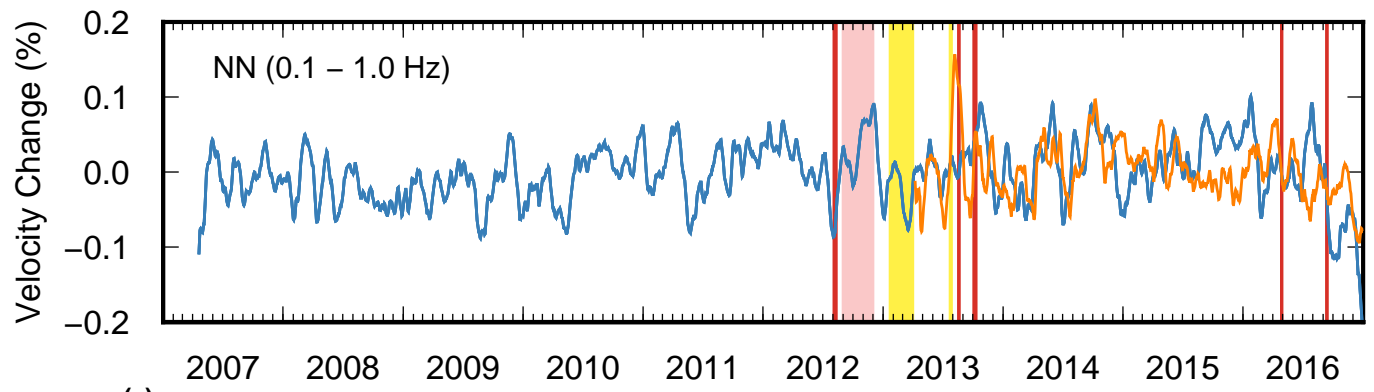

(c)

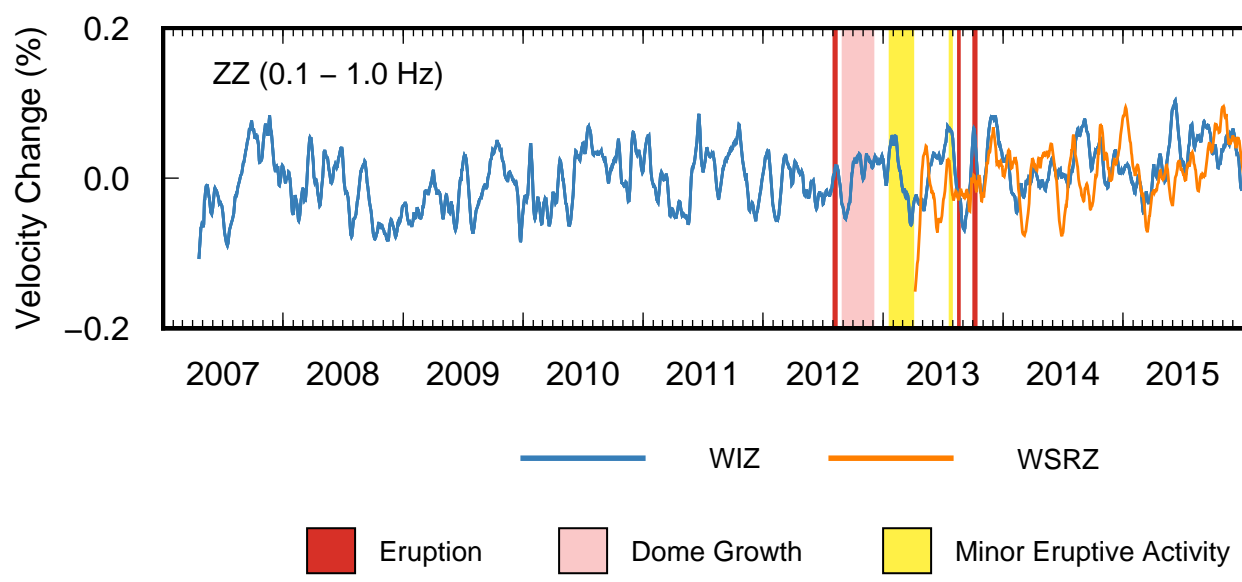

Figure B.1: Individual component-pairs that make up the averaged velocity change for 0.1-1.0 Hz auto-correlations (AC) at WIZ and WSRZ stations. Velocities are smoothed with a 30-day centered moving-window. (a) Component-pair EE. (b) Component-pair NN. (c) Component-pair ZZ. 
(a)

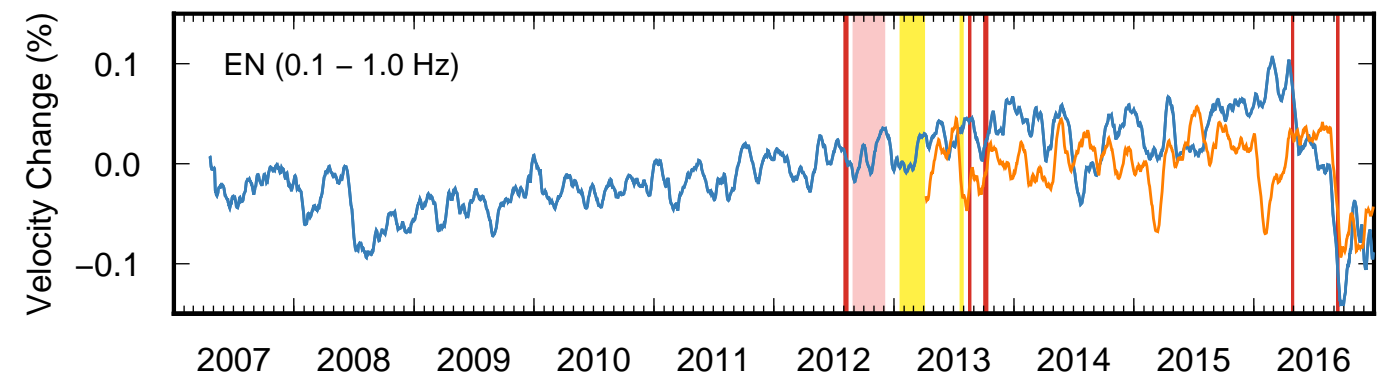

(b)

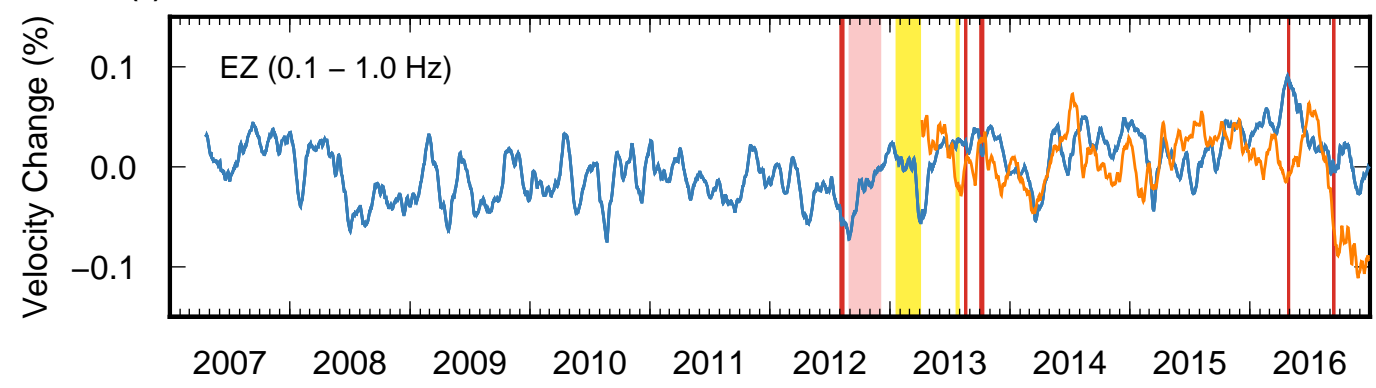

(c)

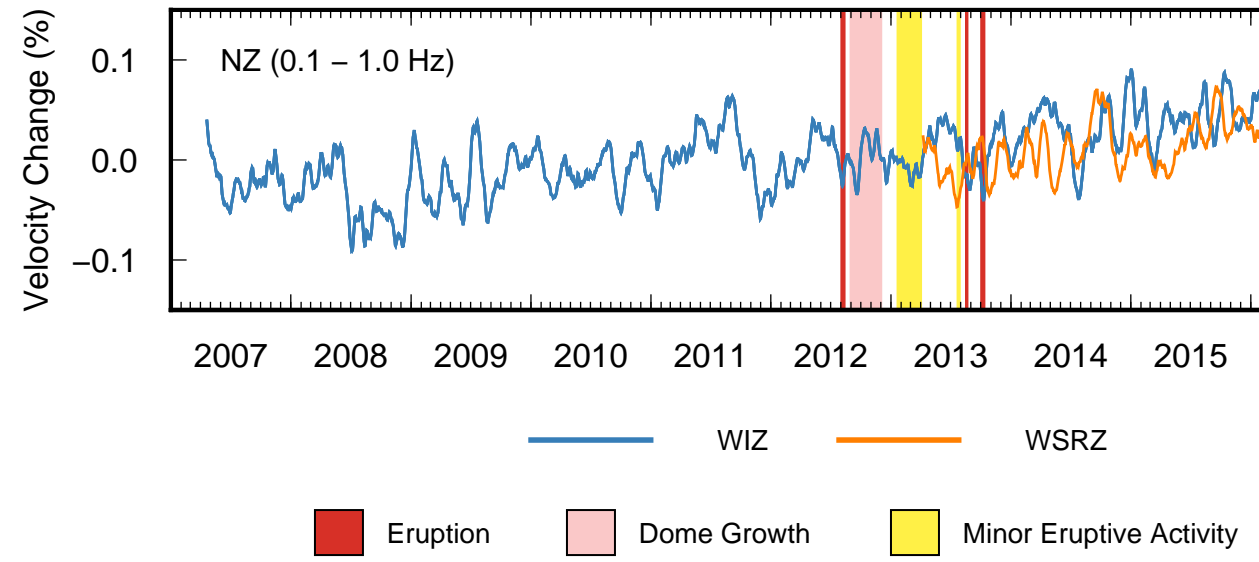

Figure B.2: Individual component-pairs that make up the averaged velocity change for 0.1-1.0 Hz cross-components (SC) at WIZ and WSRZ stations. Velocities are smoothed with a 30-day centered moving-window. (a) Component-pair EN. (b) Component-pair EZ. (c) Component-pair NZ. 
(a)

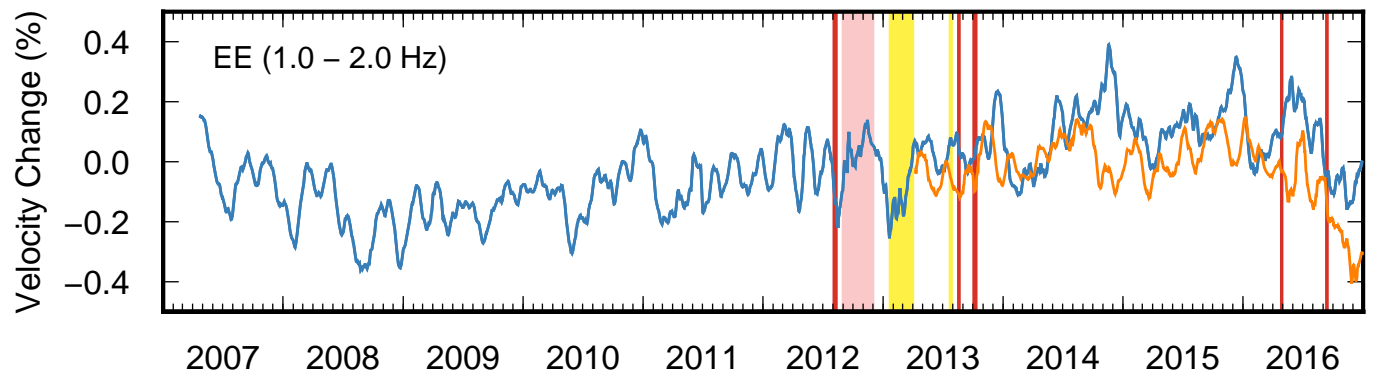

(b)

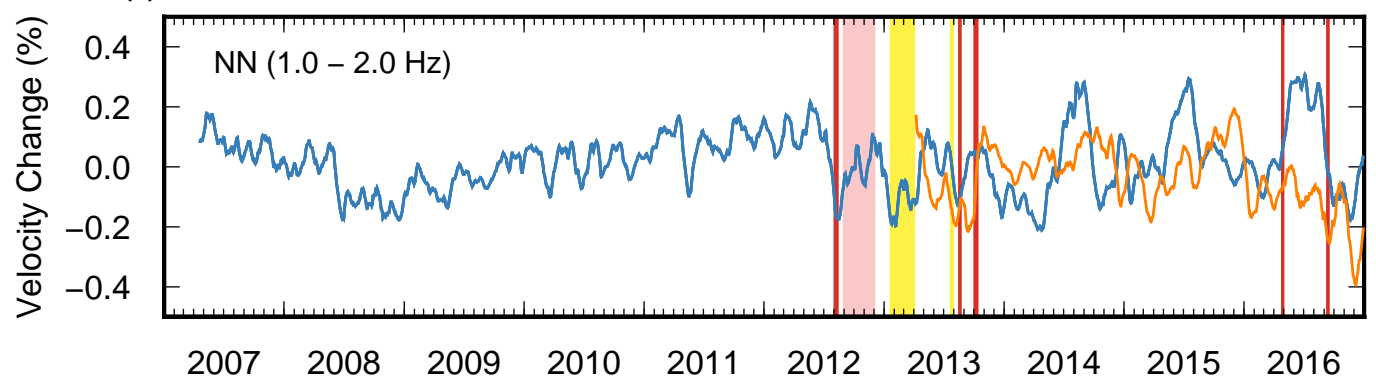

(c)
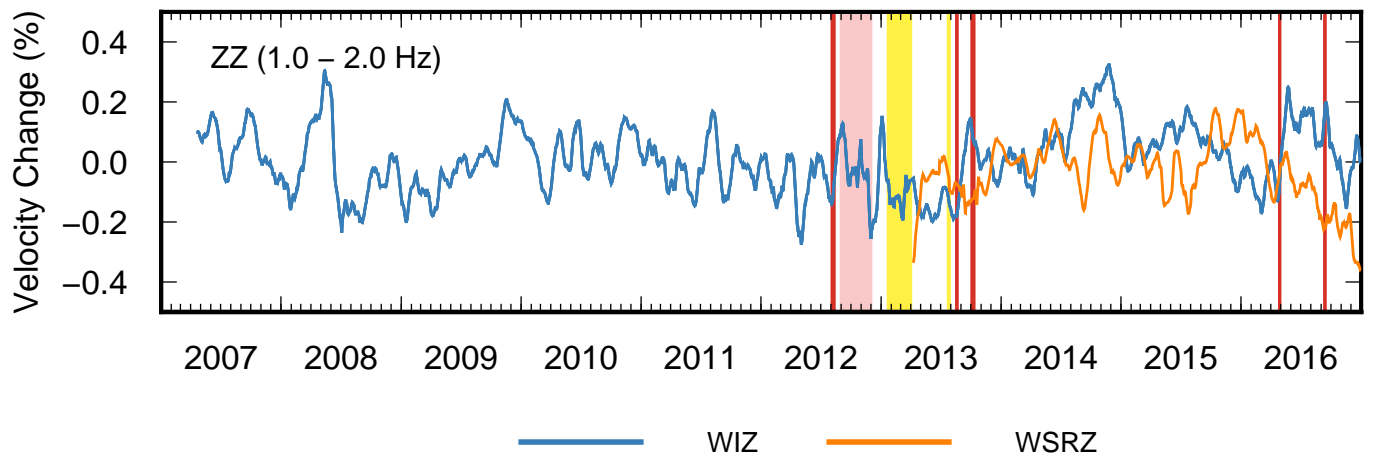

Eruption

Dome Growth

Minor Eruptive Activity

Figure B.3: Individual component-pairs that make up the averaged velocity change for $1.0-2.0 \mathrm{~Hz}$ auto-correlations (AC) at WIZ and WSRZ stations. Velocities are smoothed with a 30-day centered moving-window. (a) Component-pair EE. (b) Component-pair NN. (c) Component-pair ZZ. 


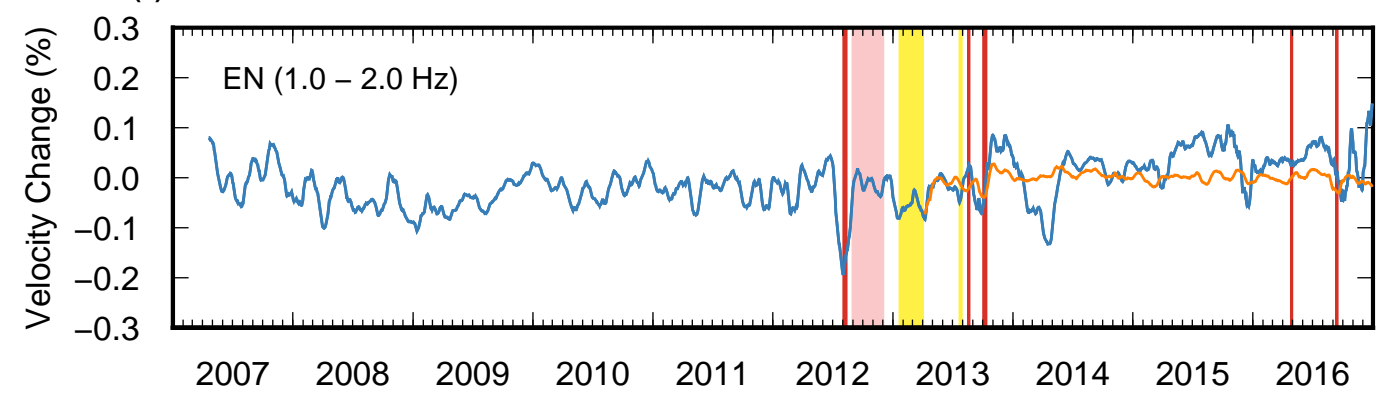

(b)

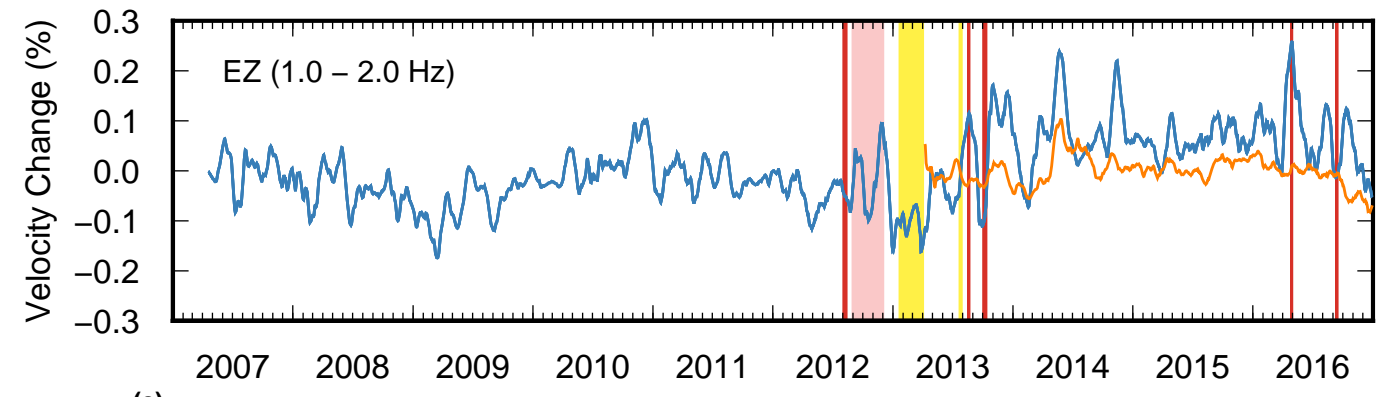

(c)

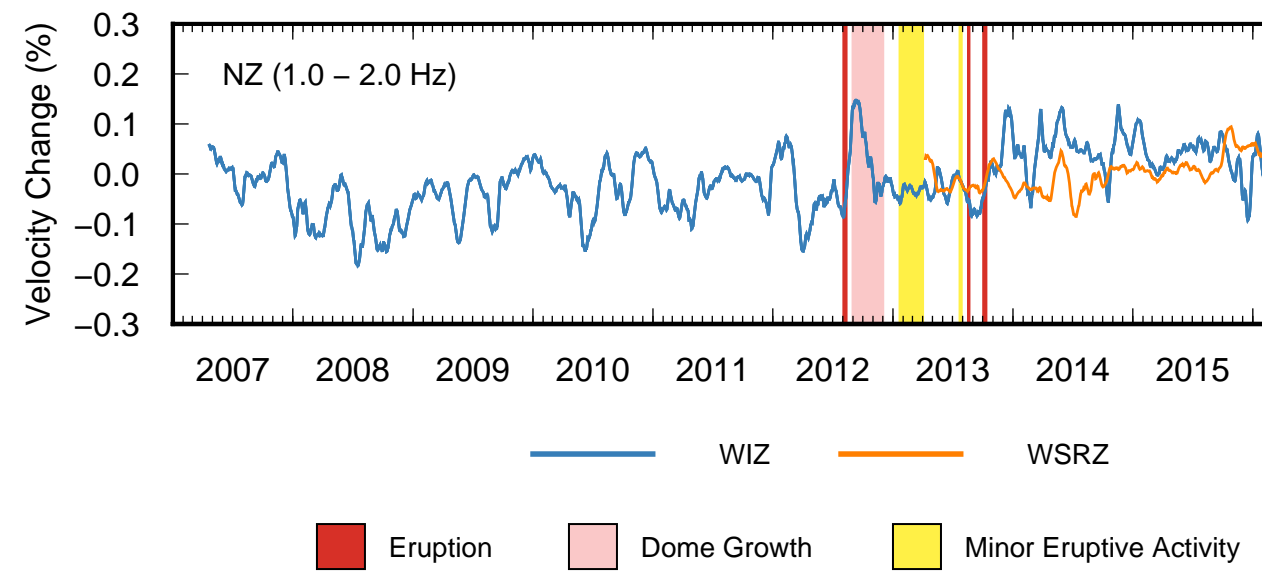

Figure B.4: Individual component-pairs that make up the averaged velocity change for 1.0-2.0 $\mathrm{Hz}$ cross-components (SC) at WIZ and WSRZ stations. Velocities are smoothed with a 30-day centered moving-window. (a) Component-pair EN. (b) Component-pair EZ. (c) Component-pair NZ. 


\section{B.2 Station-pair WIZ-WSRZ}

Figure B.5 shows the results for station-pair WIZ-WSRZ. This was processed using the same parameters as the $1.0-2.0 \mathrm{~Hz}$ single-station dataset.

(a) WIZ - WSRZ

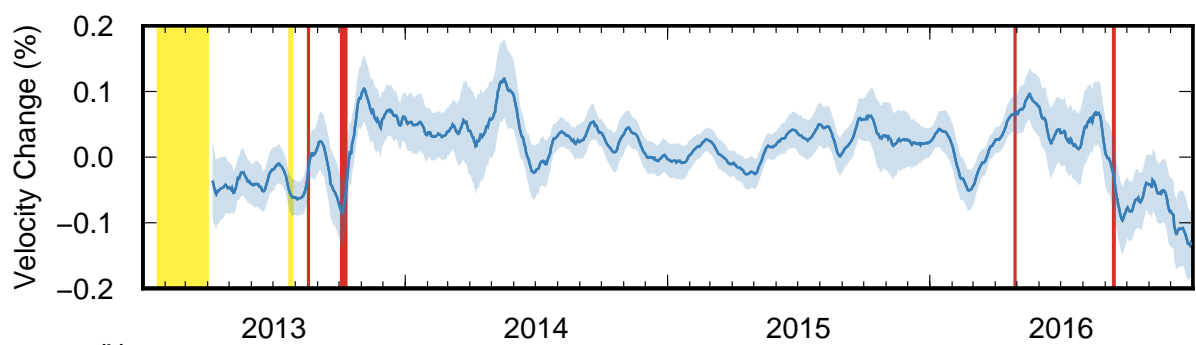

(b)

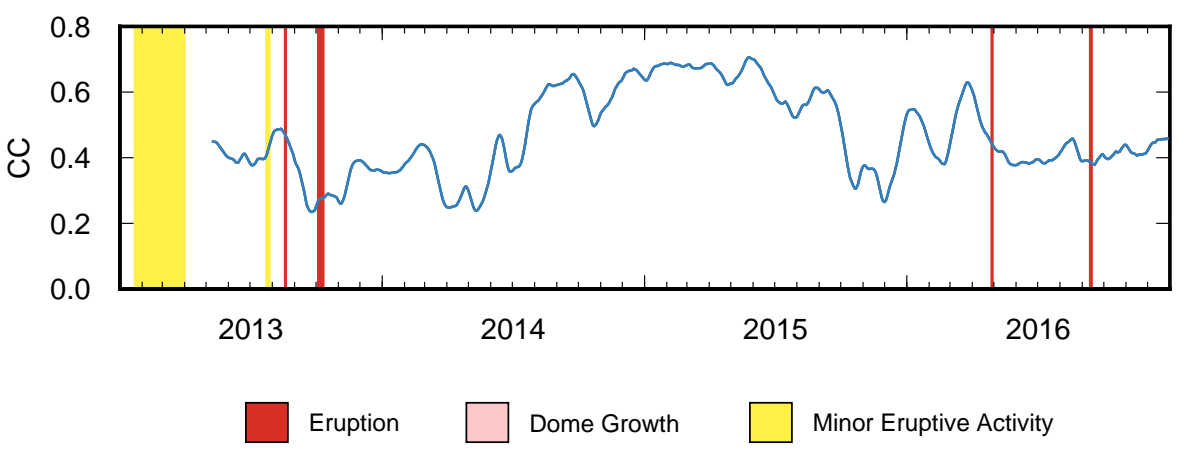

Figure B.5: Velocity changes and correlation coefficient computed for station-pair WIZ-WSRZ. These are smoothed using a 30-day window. (a) Velocity changes. (b) Correlation coefficient. 


\section{B.3 OPRZ station (Single-station Ambient Noise)}

OPRZ station was excluded when processing onshore single-station data. This decision was made due to the presense of high amplitude, long-term, changes that were not representative of the other onshore stations and therefore heavily biased the averaged time series. These changes are shown in Figure B.6 where, for 1.0-2.0 Hz data in particular, velocities vary strongly from their equivalents at other onshore stations.

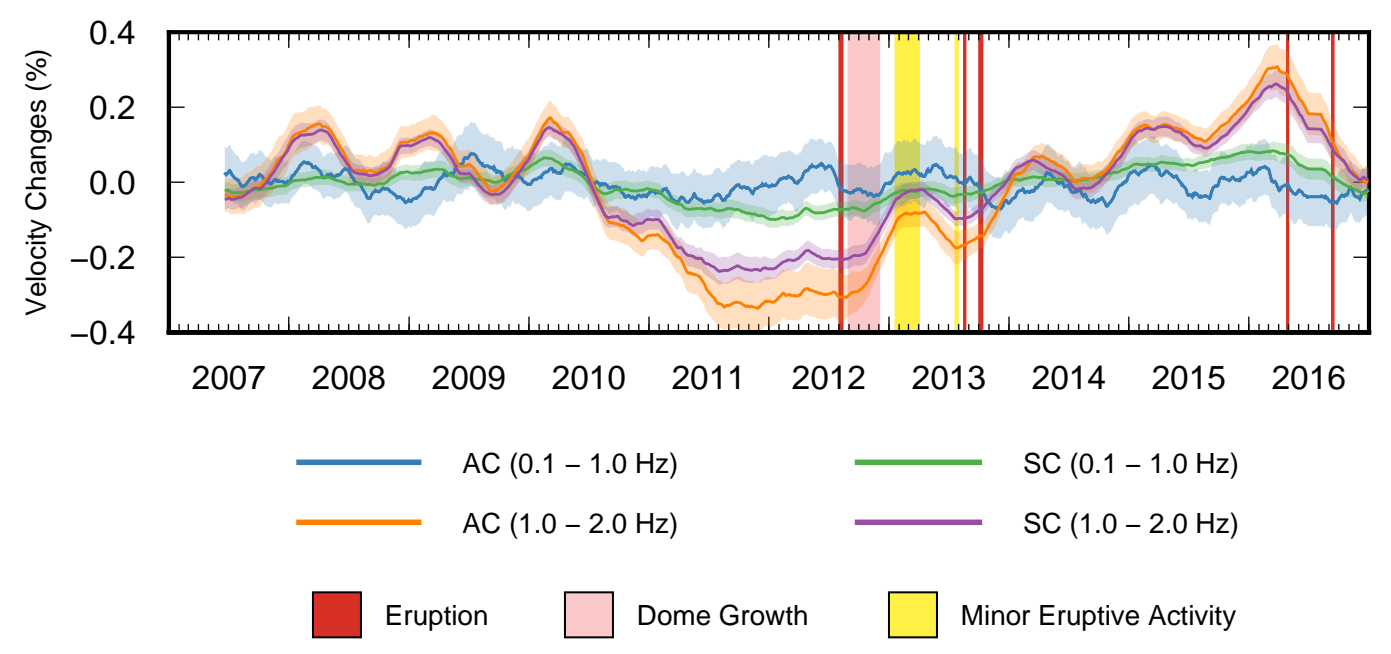

Figure B.6: Plot of velocity changes associated with single-station processing of autocorrelations (AC) and cross-components (SC) at OPRZ station. A 180-day moving window smooth is applied to all time series 